\title{
Tumorantigen-Specific CD40B Cells: Combining Enhanced Antigen-Presentation and Antibody-Secretion for Tumor Targeting
}

\author{
Inaugural-Dissertation \\ zur \\ Erlangung des Doktorgrades \\ der Mathematisch-Naturwissenschaftlichen Fakultät \\ der Universität zu Köln \\ vorgelegt von \\ Kerstin Wennhold \\ aus Köln
}

Köln 2015 
Berichterstatter:

Prof. Dr. Thorsten Hoppe

Prof. Dr. Hinrich Abken

Tag der mündlichen Prüfung:

16. April 2015 
Für meine Eltern 

Yippie-Ya-Yeah, Schweinebacke! John McClane 



\section{Danksagung}

Bedanken möchte ich mich bei allen, die zum Erfolg dieser Arbeit beigetragen und mich unterstüzt haben.

Mein Dank gilt Prof. Hallek, unter dessen Schirmherrschaft diese Arbeit entstand. Ebenso möchte ich Prof. Hoppe danken, dass er so kurzfristig meine Betreuung übernommen hat. Vielen Dank an Prof. Hinrich Abken, der meine Arbeit über die Jahre inhaltlich begleitet und unterstützt hat.

Dank geht an die Else-Kröner Fresenius Stiftung, den NZT.NRW Verbund und Köln Fortune für die Finanzierung dieses Projekts. Ebenso danke ich dem Graduiertenprogramm der Uniklinik Köln und der Bayer Health Care AG für finanzielle Unterstüzung bei Fortbildungen und Konferenzen und den wissenschaftlichen Austausch.

Mein größter Dank gilt Prof. Michael von Bergwelt-Baildon, der mir die Möglichkeit gab in seinem Labor zu forschen und meine Doktorarbeit anzufertigen. Vielen Dank für Deine Betreuung und Unterstüzung, die enthusiastischen Diskussionen und die lehrreichen Herausforderrungen. Besonderer Dank gilt Dr. Alexander Shimabukuro-Vornhagen, der mir gutes wissenschaftliches Arbeiten und die Fähigkeit meine Ergebnisse zu hinterfragen beibrachte und mir immer mit Rat zur Seite stand. Mit Deiner zuversichtlichen Art und Deinen schlauen Lösungansätzen hast Du mich immer aufgemuntert, wenn meine Experimente nicht so wollten wie ich.

Maria y Juliane, les agradezco por su amistad y el intercambio cultural. Tener que trabajar es mucho más divertido cuando se puede pasar con amigos. No importa dónde estemos en el futuro; ustedes siempre tienen un lugar especial en mi corazón.

Ich möchte mich ganz herzlich bei Martin und Sabrina für ihre tatkräftige, maustechnische und moralische Unterstützung im letzten Jahr bedanken. Ohne Eure große Hilfe im Labor hätte die Krebszelle viel häufiger auf der schlechten Seite gestanden. Vielen Dank an Anne; durch Dich habe ich endlich gelernt zu kompensieren. Michael, Maria, Juliane, Martin, Sabrina, Anne, Hans S., Hans B., Sebastian, Alex, Sasha, Geothy, Steffi und Claudia, ich danke Euch für die tolle Zeit im Labor, für die unterhaltsamen Mittagspausen, gemeinsamen Abendessen und spektakulären Laborausflüge. Ich werde von dieser besondere Erfahrung vor allem die Zeit mit Euch immer in guter Erinnerung behalten.

Ein ganz großer Dank gilt auch Hannah, Manuel, Verena und Carola. So viele Jahre begleitet Ihr mich schon durch alle Höhen und Tiefen mit Lachen, Zuhören, Quatschen und Quatsch machen, Trösten, Freuen und Fröhlich sein.

Ganz besonders bedanken möchte ich mich bei meiner Familie. Ihr seid in allen Lebenslagen bedingungslos für mich da, um Euch mit mir zu freuen oder mir mit Rat zur Seite zu stehen. Euer Vertrauen in mich haben mich bis hierher geführt.

Tim, ich danke Dir für Deine uneingeschränkte Unterstützung, Deine Geduld, Deine Einzigartigkeit, Dein grenzenloses Wissen und den Spaß, den wir zusammen haben. 



\section{Zusammenfassung}

Voraussetzung für die Induktion einer effizienten Immunantwort ist neben dem richtigen Antigen eine effiziente Präsentation von Antigen in vitro und in vivo. Dendritische Zellen (DC) gelten als die am besten charakterisierten antigen-präsentierenden Zellen. Allerdings können sie kaum in großen Mengen und ausreichender Reinheit hergestellt werden, was einen entscheidenden Nachteil bei der Nutzung von DCs in der klinischen Anwendung dar stellt. Deshalb wurde ein alternativer Ansatz entwickelt, bei dem polyklonale B-Zellen durch die Behandlung mit stimulierenden Zytokinen und dem CD40-Liganden als antigenpräsentierende Zellen genutzt werden können. Die Aktivierung durch den CD40Liganden induziert entscheidend die Antigen-präsentation, was zu einer effizienten Induktion von CD4+ und CD8+ T-Zellen in vitro führt. Darüber hinaus wandern diese CD40aktivierten (CD40)B-Zellen nicht nur in die peripheren lymphatischen Organe, sondern induzieren auch eine anti-tumorale Immunität in Mäusen.

Die Antigen-präsentation und -prozessierung durch antigen-spezifische B-Zellen ist bis zu tausendfach effektiver als bei polyklonalen B-Zellen. Deshalb wurden in der vorliegenden Arbeit antigen-spezifische B-Zellen genutzt, um die antigen-präsentierende Funktion von CD40B-Zellen zu steigern. Nach CD40-Stimulation regulieren aufgereinigte, antigenspezifische B-Zellen kostimulatorische Moleküle wie CD80 und CD86 signifikant hoch, was zu einer verstärkten Antigen-präsentation und der Induktion einer spezifischen T-Zellantwort in vitro und in vivo führt. Antigen-spezifische CD40B-Zellen induzieren eine signifikant stärkere T-Zellanwort als polyklonale CD40B-Zellen, die mit einer DC-induzieren Reaktion vergleichbar ist. Eine präventive Vakzinierung mit antigen-spezifischen CD40B-Zellen alleine oder in Kombination mit antikörper-sezernierenden Plasmazellen führt zu einer Anti-Tumor Immunität in vivo, bei der das Tumorwachstum komplett unterdrückt wird. Darüber hinaus migrieren antigen-spezifische CD40B-Zellen in den Tumor, was neue Anwendungsmöglichkeiten als Trägerstoff bei Imagingverfahren oder zum ,Drug Delivery‘ ermöglicht.

Diese Ergebnisse liefern neue Einblicke in die Rolle von aktivierten antigen-spezifischen B-Zellen als antigen-präsentierende Zellen und ihrer Nutzung in der Immuntherapie. 



\section{Abstract}

Efficient antigen presentation is a prerequisite for the development of a T-cell-mediated immune response in vitro and in vivo. As "nature's adjuvant", dendritic cells (DCs) are the most prominent professional antigen-presenting cells (APCs). However, they have several significant disadvantages concerning application as an adjuvant in cancer immunotherapy. They are rare in peripheral blood and the isolated population is not homogenous, since it also contains unwanted tolerogenic DCs. Therefore, an alternative approach to DCs was developed, in which polyclonal B cells can serve as potent APCs by treatment with the inflammatory cytokine IL-4 and the CD40 ligand. CD40-activation dramatically improves antigen presentation by normal and malignant B cells, efficiently inducing naïve and memory CD4+ and CD8+ T-cell responses. Moreover, these CD40-activated (CD40) B cells do not only home to secondary lymphoid organs, but also induce anti-tumor immunity in mice.

However, antigen-processing and -presentation by antigen-specific B cells is 1000 -fold more effective compared to polyclonal B cells. Therefore, tumorantigen-specific B cells were used in the present study to improve the antigen-presenting function of CD4OB cells. Purified tumorantigen-specific B cells highly upregulate activation markers upon CD40-stimulation resulting in an enhanced antigen-presentation and a specific T-cell response in vitro and in vivo. The T cell response elicited by antigen-specific CD40B cells is significantly stronger than that induced by polyclonal $\mathrm{CD} 40 \mathrm{~B}$ cells and comparable to the stimulation induced by mature DCs. Alone or in combination with antibody-secreting plasma cells, preventive vaccination with these antigen-specific CD40B cells leads to an anti-tumor immune response in vivo resulting in complete inhibition of tumor growth. Moreover, antigen-specific B cells home to the tumor site, thereby offering new application perspectives as vehicle for tumor imaging and drug delivery. These results provide new insights into the role of activated antigen-specific B cells as APCs and their use for cancer immunotherapy. 



\section{Abbreviations}

\begin{tabular}{|c|c|}
\hline$A_{500}$ & Absorbance at $500 \mathrm{~nm}$ \\
\hline APC & antigen-presenting cells \\
\hline BCR & B cell receptor \\
\hline$\beta-M E$ & beta-mercaptoethanol \\
\hline BSA & bovine serum albumin \\
\hline${ }^{\circ} \mathrm{C}$ & degrees Celsius \\
\hline CCR & C-C-chemokine receptor \\
\hline$C D$ & cluster of differentiation \\
\hline CD40B & CD40-activated B cells \\
\hline CD40L & CD40 ligand \\
\hline CEA & cell adhesion molecule \\
\hline CFSE & 5-(and-6)-carboxyfluorescein diacetate succinimidyl ester \\
\hline $\mathrm{cm}$ & centimeter \\
\hline $\mathrm{CO}_{2}$ & carbon dioxide \\
\hline CTLA-4 & cytotoxic T-lymphocyte-associated Protein 4 \\
\hline CXCR & CXC-motiv-chemokine receptor \\
\hline DC & dendritic cell \\
\hline DMSO & dimethylsulfoxid \\
\hline EDTA & ethylenediaminetetraacetic acid \\
\hline ELISA & Enzyme Linked Immunosorbent Assay \\
\hline ER & endoplasmatic riticulum \\
\hline FACS & fluorescence activated cell sorting \\
\hline FcR & Fc receptor \\
\hline FDA & Food and Drug Administration \\
\hline FITC & Fluorescein isothiocyanate \\
\hline FBS & fetal bovine serum \\
\hline g & gram \\
\hline GC & germinal center \\
\hline GM-CSF & granulocyte/macrophage colony stimulating factor \\
\hline Gy & gray \\
\hline h & hour \\
\hline $\mathrm{H}_{2} \mathrm{O}$ & water \\
\hline HBV & hepatitis-B-antigen \\
\hline HIV & Human Immundeficient-Virus \\
\hline HLA & human leukocyte antigen \\
\hline HSC & Hematopoietic stem cells \\
\hline IFA & Incomplete Freund's adjuvant \\
\hline IFN & interferon \\
\hline $\lg$ & immunoglobulin \\
\hline i.p. & intraperitoneal \\
\hline i.v. & intravenous \\
\hline IL & interleukin \\
\hline kDa & kilo Dalton \\
\hline KLH & Keyhole Limpet Hemocyanin \\
\hline LN & lymph node \\
\hline
\end{tabular}




\begin{tabular}{|c|c|}
\hline LPS & Lipopolysaccharide \\
\hline Luc & Luciferase \\
\hline M & molar \\
\hline $\mathrm{mAb}$ & monoclonal antibody \\
\hline MAGE & melanoma-antigen family \\
\hline $\mathrm{MFI}$ & mean fluorescent intensity \\
\hline $\mathrm{mg}$ & milligram \\
\hline $\mathrm{MHC}$ & major histocompatibility complex \\
\hline $\min$ & minutes \\
\hline MLR & mixed-lymphocyte reaction \\
\hline $\mathrm{mmol}$ & millimol \\
\hline $\mathrm{mM}$ & millimolar \\
\hline $\mathrm{ml}$ & milliliter \\
\hline $\mathrm{mm}$ & millimeter \\
\hline MW & molecular weight \\
\hline $\mathrm{MZ}$ & marginal zone \\
\hline$\mu g$ & microgram \\
\hline$\mu l$ & microliter \\
\hline$\mu \mathrm{m}$ & micrometer \\
\hline$\mu \mathrm{M}$ & micromolar \\
\hline ng & nanogram \\
\hline NK & natural killer \\
\hline $\mathrm{nm}$ & nanometer \\
\hline $\mathrm{nM}$ & nanomolar \\
\hline OVA & chicken ovalbumin \\
\hline PBMC & peripheral blood mononuclear cell \\
\hline PBS & phosphate buffered saline \\
\hline PC & plasma cell \\
\hline PD-L1 & Programmed death-ligand 1 \\
\hline PE & phycoerythrin \\
\hline $\mathrm{PE}-\mathrm{Cy}$ & phycoerythrin- cyanine \\
\hline Pen/Strep & Penicillin/ Streptomycin \\
\hline PerCp-Cy & peridinin chlorophyll protein complex-cyanine dye \\
\hline PFA & paraformaldehyde \\
\hline rh & recombinant human \\
\hline $\mathrm{rm}$ & recombinant murine \\
\hline RT & room temperature \\
\hline s.c. & subcutaneous \\
\hline SD & standard deviation \\
\hline SDS & sodium dodecylsulfate \\
\hline SEM & standard error of mean \\
\hline TCR & T cell receptor \\
\hline TGF $\beta$ & transforming growth factor-beta \\
\hline$T_{h}$ & Thelper cell \\
\hline TNF & tumor necrosis factor \\
\hline Treg & regulatory T cell \\
\hline$U$ & units \\
\hline
\end{tabular}




\section{Table of Contents}

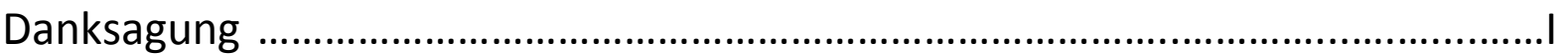

Zusammenfassung ........................................................................................

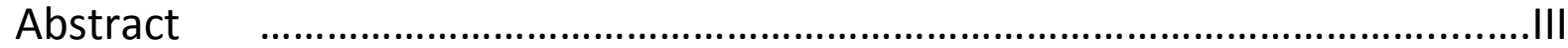

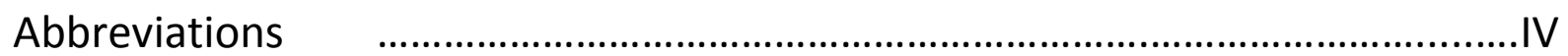

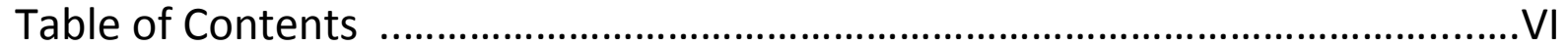

INTRODUCTION 1

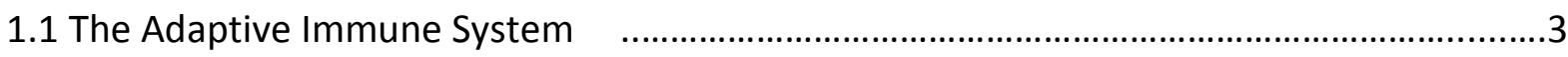

1.1.1 T Cells

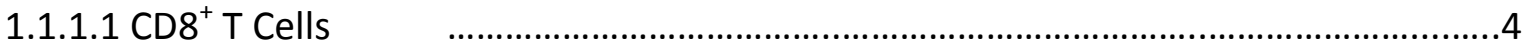

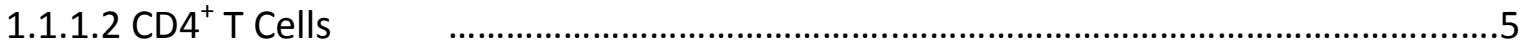

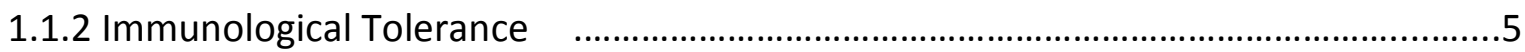

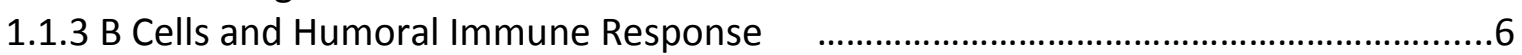

1.1.4 Antigen-Presentation to T cells

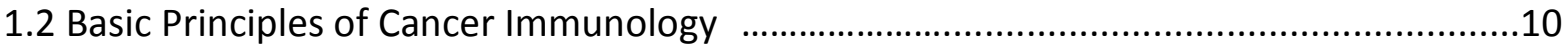

1.2.1 Cancer Immunosurveillance

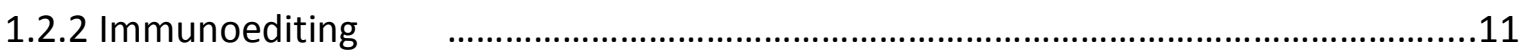

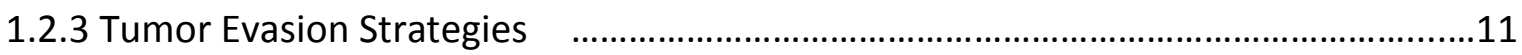

1.3 Targeted Immunotherapy

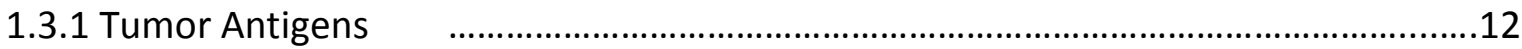

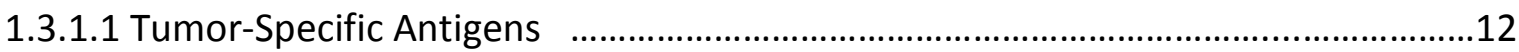

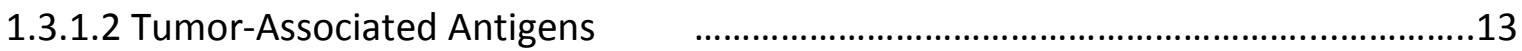

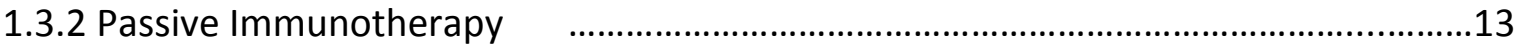

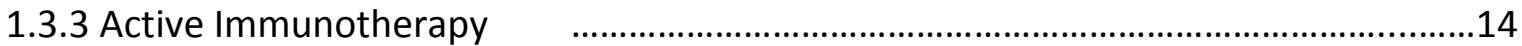

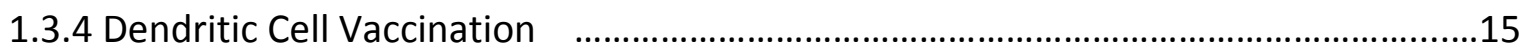

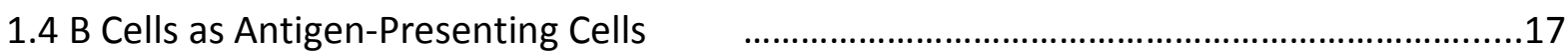

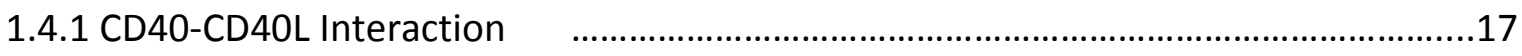

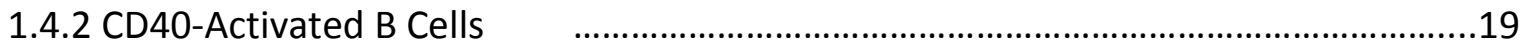

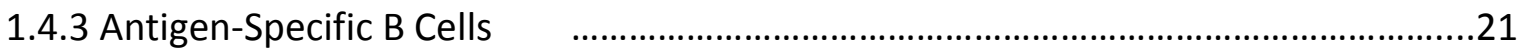

1.5 Purpose of this Study

MATERIAL AND METHODS 23

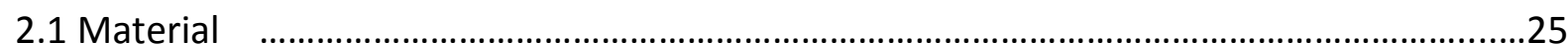

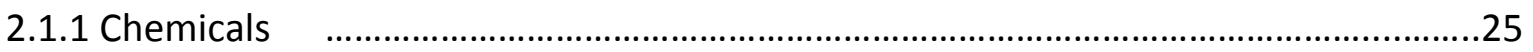

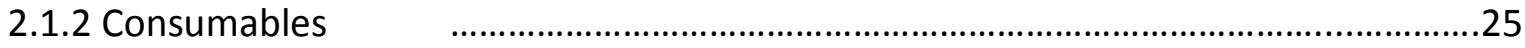

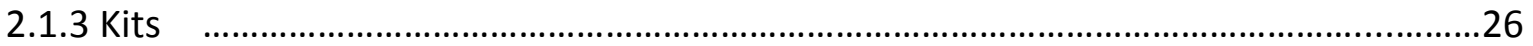

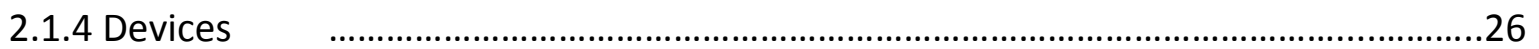

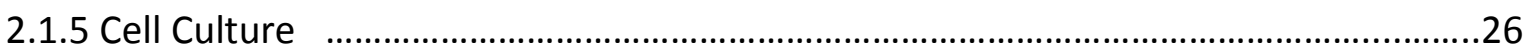

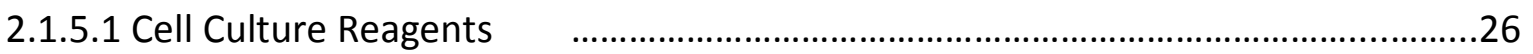

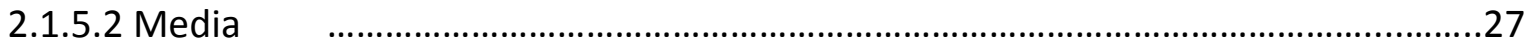

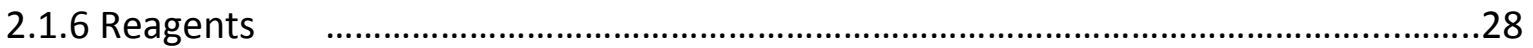


2.1.7 Solutions and Buffers

2.1.8 Software

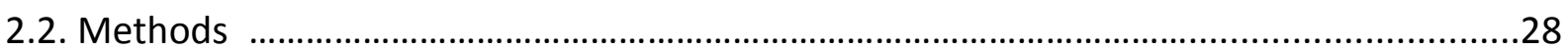

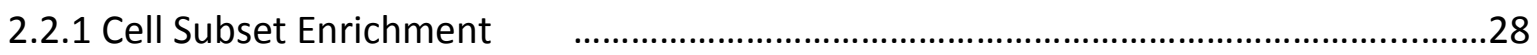

2.2.1.1 Purification of Murine Lymphocytes from Spleen $\quad$.........................................28

2.2.1.2 Purification of Murine Lymphocytes from Lymph Nodes $\quad$..............................29

2.2.1.3 Purification of Human Lymphocytes from Blood $\quad$..........................................29

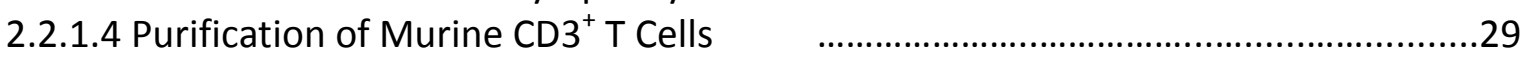

2.2.1.5 Purification of $\mathrm{CD} 34^{+}$Bone Marrow Progenitor Cells $\quad$............................................29

2.2.1.6 Purification of Murine and Human CD19 ${ }^{+}$B Cells

2.2.1.7 Purification of Murine and Human Antigen-Specific B Cells $\quad$.................................31

2.2.1.7.1 Generation of Protein-Biotin Conjugates for Antigen Tetramers ................31

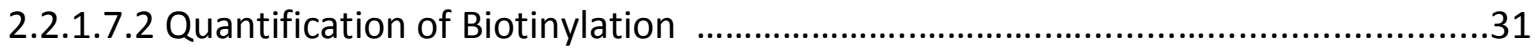

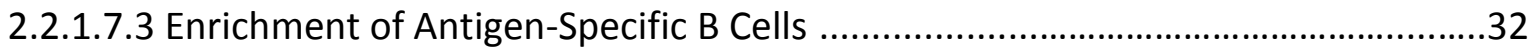

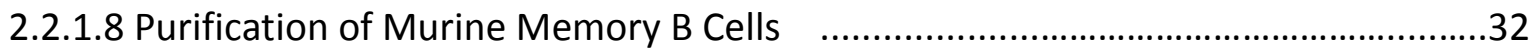

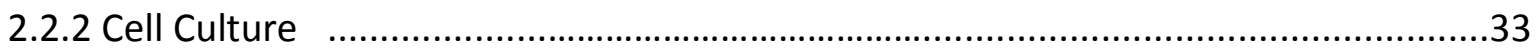

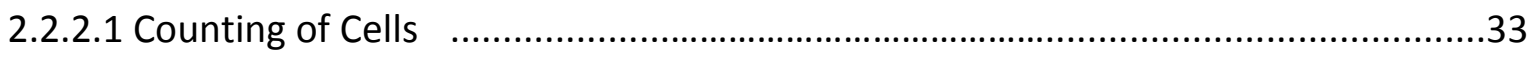

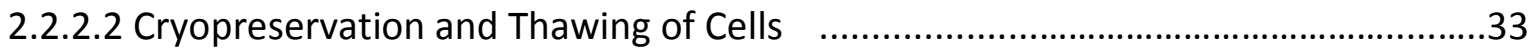

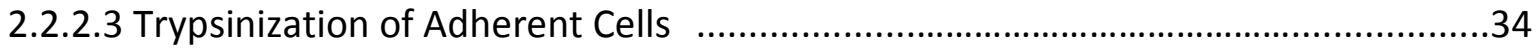

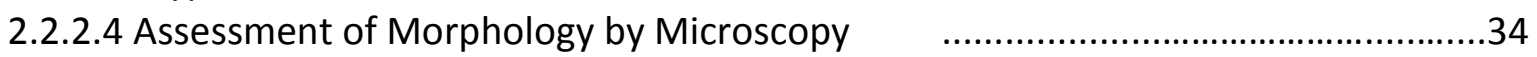

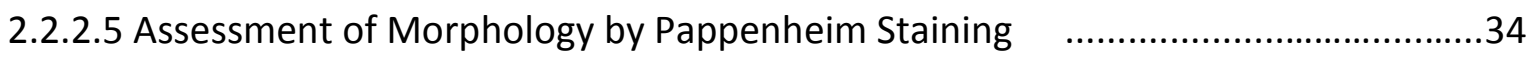

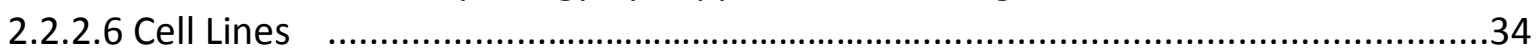

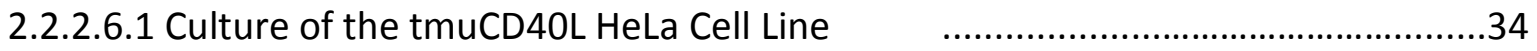

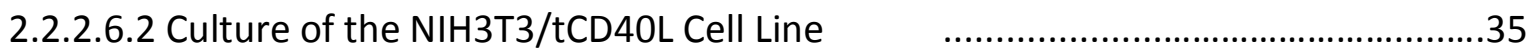

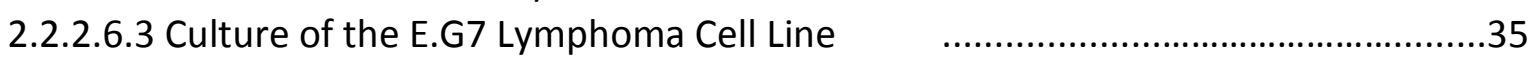

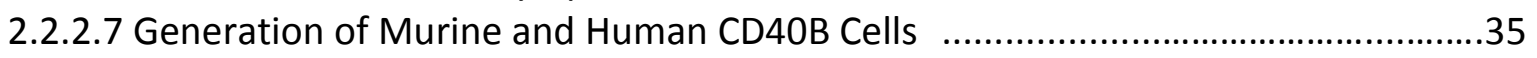

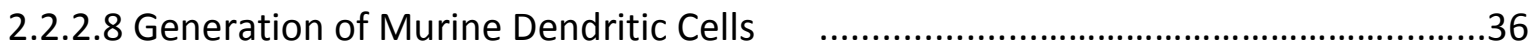

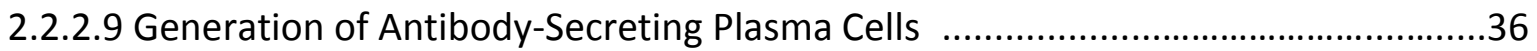

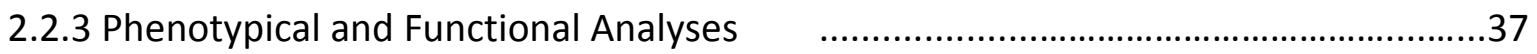

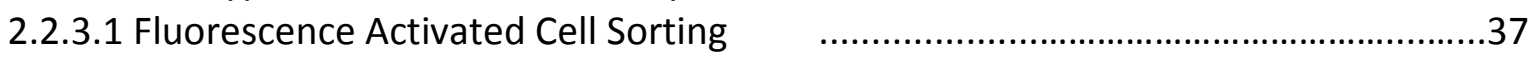

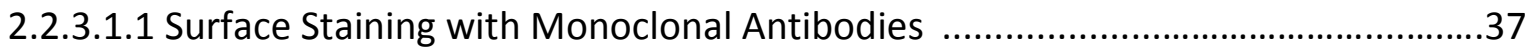

2.2.3.1.2 Staining of Antigen-Specific B Cells with Antigen Tetramers ..........................38

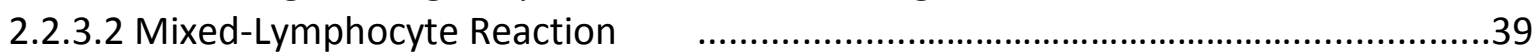

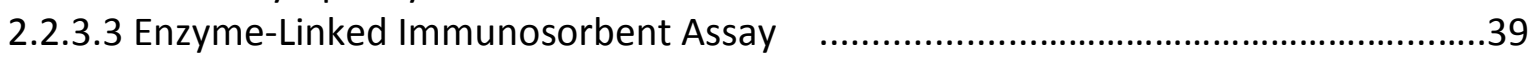

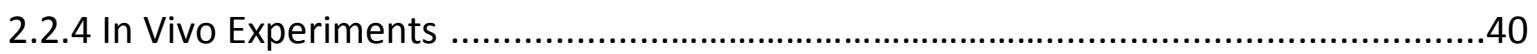

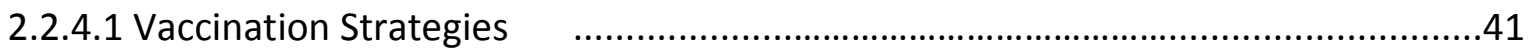

2.2.4.1.1 Immunization with Peptide-Loaded Cell Subsets $\quad$........................................41

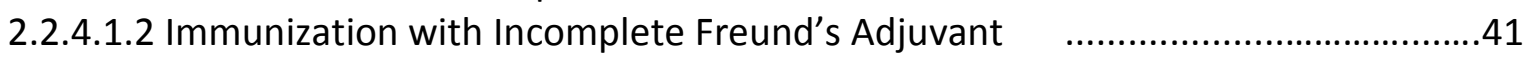

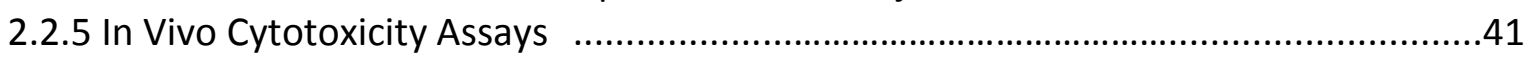

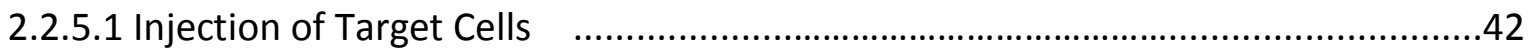

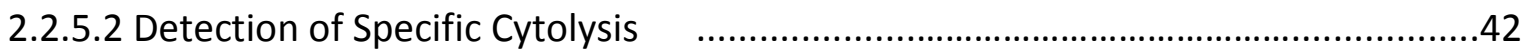

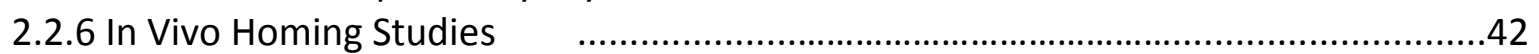

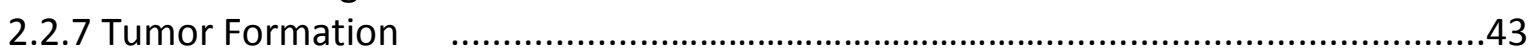

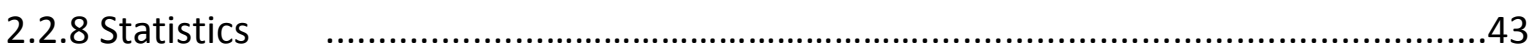


RESULTS $\quad 45$

3.1 Antigen-Specific B Cells can be Isolated by Antigen Tetramers ..................................47

3.1.1 Murine Antigen-Specific B Cells can be Purified by Antigen-Tetramers ................47

3.1.2 Murine Antigen-Specific B Cells Show a Class-Switched Phenotype $\quad$.................50

3.1.3 Efficient Purification of Human Antigen-Specific B Cells Depends on Initial HBV-Specific B Cell Frequency …........................................................56

3.1.4 Human Antigen-Specific B Cells Show a Class-Switched Phenotype $\quad$.................58

3.2 Stimulation with the CD40 Ligand Activated Antigen-Specific B Cells ..........................65

3.2.1 Murine Antigen-Specific B Cells Show a Proliferative Disadvantage in the CD4OL System

3.2.2 Murine Antigen-Specific B Cells Upregulate Activation Markers

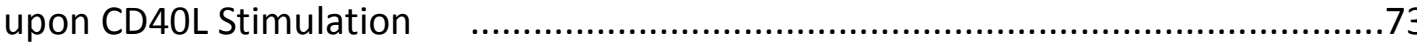

3.2.3 Human Antigen-Specific B Cells are Activated by Stimulation with the CD40L........78

3.3 Murine Antigen-Specific CD40B Cells Efficiently Present Antigen to T Cells In Vitro.......83

3.4 Antigen-Specific CD40B Cells Migrate to Secondary Lymphoid Organs and to the Tumor

3.5 Antigen-Specific CD40B Cells Induce an Antigen-Specific Immune Response In Vivo....100

3.6 Antigen-Specific B Cells Differentiate into Antibody-Secreting B Cells upon Stimulation .102

3.7 The Combined Vaccination with Antigen-Specific CD40B Cells and

Plasma Cells Induces Anti-Tumor Immunity and Prolongs Survival $\quad$..........................106

DISCUSSION

4.1 Murine Antigen-Specific B Cells are Class-Switched and Develop an APC Phenotype when Stimulated with the CD40L

4.2 Human Antigen-Specific B Cells can be Isolated from PBMCs and Develop an APC Phenotype when Stimulated with the CD40L

4.3 Murine Antigen-Specific CD40B cells Induce an Antigen-Specific

T Cell Response In Vitro and In Vivo

4.4 Tumorantigen-Specific CD40B Cells Home to Secondary Lymphoid Organs and to the Tumor

4.5 Antigen-Specific B Cells can be Stimulated to Differentiate into Antibody-Secreting Plasma Cells

4.6 Immunotherapy with Antigen-Specific CD40B Cells in Combination With Plasma Cells Leads to Anti-Tumor Immunity and Prolongs Survival 124

4.7 Concluding Remarks

REFERENCES 

Introduction 



\section{Introduction}

The immune system plays an essential role in the development and progression of tumors. Several different immune cells interact with tumor cells and the surrounding non-malignant cells of the tumor micromilieu. On the one hand, the effector immune cells prevent tumor growth and destroy established tumors. On the other hand, tolerogenic immune cells inhibit the anti-tumor immunity and enhance tumor growth by secretion of growth factors and expression of inhibitory surface molecules (Popi et al. 2004).

Immunotherapy of malignant diseases is one of the most promising therapeutic approaches in modern cancer treatment. Beside the clinically established and routinely applied therapy with antibodies, the use of tumor vaccines could further improve treatment of malignant diseases. Tumor vaccines aim to enhance preexisting immune responses or induce novel tumor-directed effector mechanisms, thereby tipping the balance towards immunological enhanced tumor control.

CD40-activated B cells provide a promising source of antigen-presenting cells for the use as cellular adjuvant in cancer immunotherapy (Schultze et al. 1997). Most published B cellbased immunotherapeutic approaches use polyclonal B cells which are loaded with tumor antigens (Wennhold et al. 2013). However, this strategy does not exploit the full potential of B cell-based cancer immunotherapy, since it does not fully exploit the advantage of antigenspecificity conferred by the $B$ cell receptor, i.e. enhanced antigen-uptake and production of antigen-specific antibodies.

\subsection{The Adaptive Immune System}

The immune system consists of specialized systems, which provide protection against invading pathogens and degenerated endogenous cells. The first line of defense is represented by the innate immune system. It is phylogenetically selected and provides immediate defense against infections. Macrophages and natural killer (NK) cells make up the cellular defense mechanism of the innate immune system. Recognition of conserved patterns on pathogens by their diverse receptors triggers macrophage activation resulting in phagocytosis of the pathogens and release of cytokines and chemokines for the attraction of other leukocytes (Mantovani and Sica 2010). NK cells on the other hand recognize the 
absence of MHC molecules as it occurs in transformed or stressed cells (Herberman et al. 1986). However, unlike the adaptive immune system it does not provide a long-lasting or protective immunity. The adaptive immune system, on the other hand, is acquired during lifetime. Unlike innate immunity, adaptive immunity is characterized by the development on immunologic memory and improves with repeated exposures to pathogens. An adaptive immune response consists of two components: the humoral and the cellular mediated immunity. The humoral immunity is mediated by antibodies produced by B cells (Kearney et al. 1997). They are soluble molecules that bind to specific antigens on the surface of their target, thereby neutralizing them or targeting them for destruction by phagocytes. Cellular immunity involves direct effector functions of $\mathrm{T}$ cells directed against intracellular pathogens, which are inaccessible to the humoral system.

\subsubsection{T Cells}

After developing in the thymus, $\mathrm{T}$ cell recirculate in the bloodstream and the lymphatic tissues as mature naïve $T$ cells until they encounter their specific antigen. The antigen is presented to them in combination with major histocompatibility complex (MHC) and costimulatory molecules (Bretscher 1999, Bevan 2004) leading to a cascade of signaling events that result in the activation of naïve T cells. The activated T cells rapidly proliferate, migrate through the tissues to the sites of antigen presence and perform effector functions, which depend on the subtype of T cells (Broere et al. 2011).

\subsubsection{1 $\mathrm{CD}^{+} \mathrm{T}$ Cells}

$\mathrm{CD}^{+}$cytotoxic T cells recognize 8-10 amino acid long peptides presented on the surface of their target cells in combination with self-MHC class I molecules (Klein and Sato 2000). Naïve $\mathrm{CD}^{+} \mathrm{T}$ cells become initially activated ('primed') when their T-cell receptor binds to its specific antigen presented by activated antigen presenting cells (APCS). The effector function of $\mathrm{CD}^{+} \mathrm{T}$ cells includes secretion of interferon (IFN)- $\gamma$ and the apoptosis inducing molecules perforin and granzyme B and the upregulation of the Fas ligand. Furthermore, interaction with the specific antigen leads to differentiation into memory $T$ cells, which respond faster upon re-exposure to the antigen (Weninger et al. 2002). 


\subsubsection{CD4 ${ }^{+} \mathrm{T}$ Cells}

In contrast to $\mathrm{CD} 8^{+} \mathrm{T}$ cells, $\mathrm{CD} 4^{+}$helper $\mathrm{T}$ cells recognize $10-34$ amino acid long peptides from exogenous proteins presented in combination with MHC class II molecules by APCs (Klein and Sato 2000). Depending on the context of antigen encounter $\mathrm{CD}^{+} \mathrm{T}$ cells can differentiate into several distinct subgroups, which can be distinguished by their cytokine profile.

Type 1 cytokine-secreting $\mathrm{CD}^{+}$helper $\mathrm{T}$ cells $\left(\mathrm{T}_{\mathrm{h}} 1\right)$ develop in the presence of IL-12 and a strong antigen-TCR interaction or high density of antigen (Fishman and Perelson 1999). $T_{h} 1$ cells by production of IFN- $\nu$ are responsible for directing cell-mediated immune responses (Mosmann et al. 1986). Furthermore, they enhance activation of APCs through engagement of the CD40 ligand (CD40L) and its receptor CD40 (Bourgeois et al. 2002). Interleukin (IL)-4 and IL-6 secretion and a weak antigen-T cell receptor (TCR) interaction or low density of antigen stimulates development of $\mathrm{T}_{\mathrm{h}} 2 \mathrm{~T}$ cells, which secrete IL-4, IL-5, IL-6, IL-10 and IL-13. $T_{h} 2$ cells thereby favor a humoral immune response by $B$ cells, while inhibiting $T_{h} 1$ cellular immune responses. Another subset of T helper cells develops in the presence of IL-23 into IL-17A secreting T cells (Th17 cells). Th17 cells are the first T cells that are generated during infection and their interaction with fibroblasts, epithelial cells and keratinocytes leads to the recruitment of neutrophils and macrophages (Steinman 2007, Stockinger and Veldhoen 2007). They also play a major role in autoimmune diseases such as rheumatoid arthritis, psoriasis and Crohn's disease.

Regulatory $\mathrm{CD}^{+}{ }^{+} \mathrm{T}$ cells (Tregs) are characterized by the constitutive expression of CD25 and the transcription factor FoxP3 (Wing and Sakaguchi 2010). They build one arm of immunological tolerance mechanisms.

\subsubsection{Immunological Tolerance}

Activation of self-reactive $T$ cells and thus failure of the immune system to tolerate the body's own tissues can result in tissue-specific autoimmunity. Two essential mechanisms account for self-non-self discrimination by the immune system, which allows productive immunity to pathogens while minimizing the risk of autoimmunity. Central tolerance includes clonal deletion of autoreactive T cells in the thymus according to their recognition pattern (positive selection) and affinity (negative selection). This results in a repertoire of 
T-cells that recognize foreign antigens presented by self-MHC molecules (Kappler et al. 1987, Kisielow et al. 1988). Peripheral tolerance deals with auto-reactive T cells, which escape clonal deletion. One mechanism is the induction of anergy in naïve $T$ cells to avoid autoimmune responses to tissue-specific proteins, which are not presented in the thymus and thus do not lead to clonal deletion of self-reactive T cells (Albert et al. 2001). Anergy is induced by antigen recognition of the TCR in the absence of costimulatory signals, as it occurs with antigens expressed on the surface of tissue cells in the periphery, and is characterized by the induction of a hyporesponsive state of self-reactive naïve T cells. More recently, evidence for the existence of a second mechanism of immune tolerance was provided by the identification and characterization of regulatory $\mathrm{T}$ cells (Fontenot et al. 2003, Sakaguchi 2005). Tregs suppress T-cell responses through direct contact with APCs or through production of anti-inflammatory cytokines such as transforming growth factor (TGF)- $\beta$, IL-10 and IL-35.

\subsubsection{B Cells and Humoral Immune Response}

B cells develop in the bone marrow from hematopoietic stem cells to common lymphoid progenitor cells to pro B cells and pre B cells. These different stages are characterized by immunoglobulin gene rearrangement, changes in gene expression and the surface phenotype (Osmond 1990, Hardy et al. 1991, Hardy 2003). Dysfunctional or autoreactive B cells recognizing self-antigens are eliminated by receptor editing (Melchers et al. 1995, Hardy et al. 2000) before immature B cells leave the bone marrow and enter the spleen. Engagement of the surface $B$ cell receptor (BCR) of the isotype $M$ and $D$ by its specific antigen leads to maturation and terminal differentiation (Osmond et al. 1998, Rolink et al. 1999)

Three different B cell subsets can be defined according to their location. B-1 B cells locate mainly in the pleural and peritoneal cavities (Kantor and Herzenberg 1993) and are involved in the early stages of thymus-independent immune responses against bacterial patterns (Allman et al. 2004). Upon BCR-signaling they can differentiate into short-lived IgMproducing plasmablasts and are responsible for the majority of the physiological IgM levels in serum (Kraal 1992, Hardy and Hayakawa 2001). 


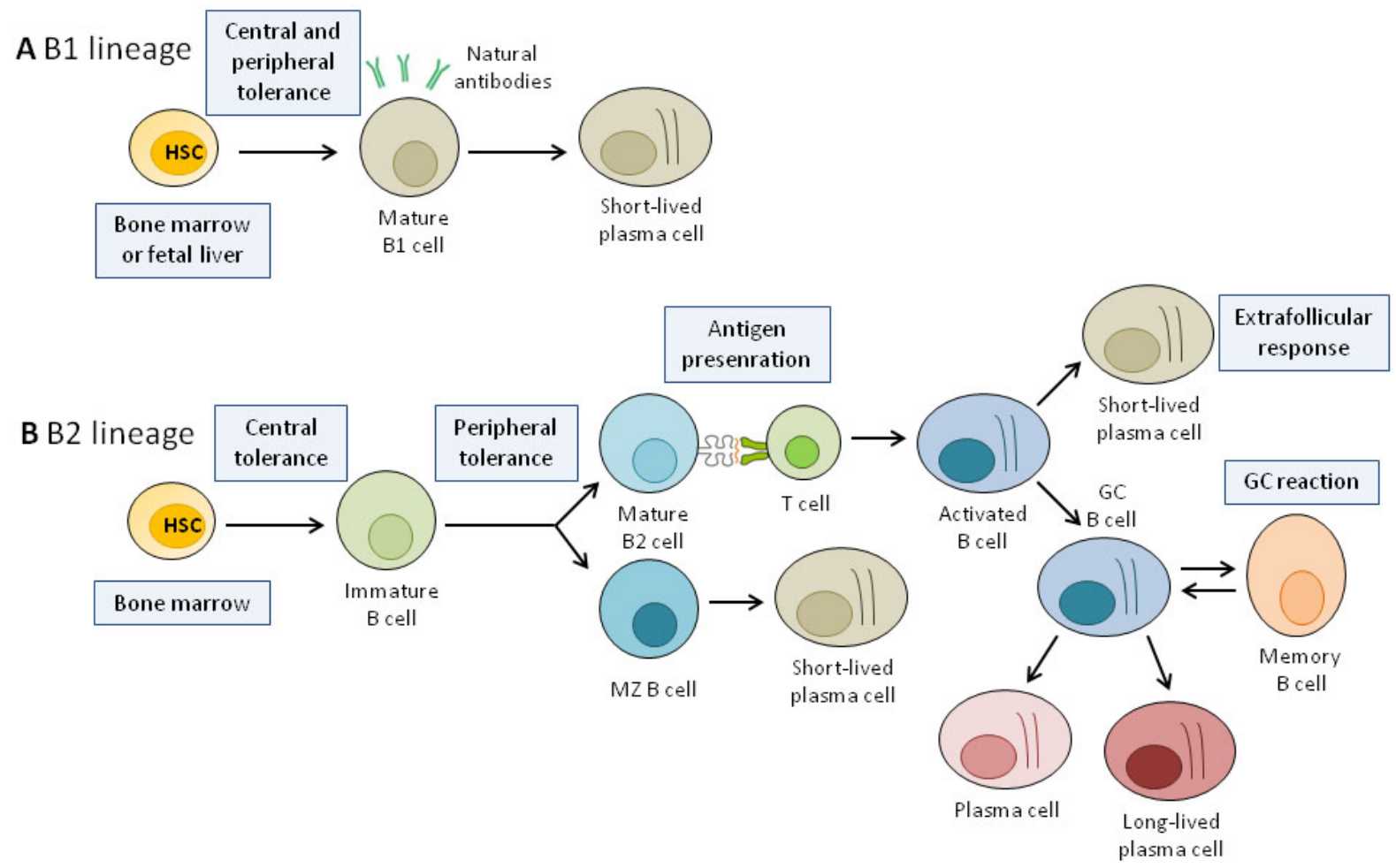

Fig. 1.1 B cell development under physiological conditions (A) B1 B cells develop from hematopoietic stem cells (HSCs) in the bone marrow or the fetal liver, differentiate into short-lived plasma cells and produce natural IgM-antibodies. (B) B2 B cells develop from HSCs in the bone marrow. Following rearrangement of their B-cell receptor and removal of autoreactive cells via central tolerance, immature B2 B cells relocate to the spleen. Those immature B2 B cells that escape the processes of peripheral tolerance differentiate into marginal zone (MZ) B cells or mature follicular B cells. Upon T-cell activation, follicular B cells develop into short-lived plasma cells or form germinal centers $(G C)$, where they differentiate into long-lived plasma cells or memory $B$ cells (Dorner et al. 2009).

Marginal zone (MZ) B cells are located in the marginal sinus in the spleen at the border of the red and the white pulp and link the innate and adaptive immune system (Martin and Kearney 2002). MZ B cells respond early to infections due to their close contact to bloodborne antigens (Kraal 1992). Upon stimulation of toll-like-receptors alone or in combination with stimulation of the $B C R, M Z B$ cells migrate to the T-cell-B-cell border of the lymphoid follicle, where they present antigen to $\mathrm{CD} 4^{+} \mathrm{T}$ cells (Attanavanich and Kearney 2004). After migration into the red pulp, they differentiate into plasma cells secreting large amounts of IgM (Martin et al. 2001). B-1 and MZ B cells do not form germinal centers and thus do not undergo somatic hypermutation. Therefore, they express only low-affinity BCRs, which mainly distinguishes them from follicular B cells.

Follicular B cells re-circulate through the blood and the lymph to B cell follicles of the lymph nodes, the spleen and Peyer's patches (Allman and Pillai 2008). Encounter of their specific 
antigen and subsequent cross-linking of the BCR leads to BCR-mediated endocytosis of the antigen, antigen processing in endosomes and its presentation in the context of MHC II molecules (Lanzavecchia 1990). Upon antigen encounter, follicular B cells change their chemokine receptor profile, migrate to the T-cell-B-cell border and bind to activated $\mathrm{CD} 4^{+} \mathrm{T}$ cells that express the corresponding TCR, building a so called "immunological synapse" (Grakoui et al. 1999, Pereira et al. 2010). Activated B cells undergo different fates after T cell encounter depending on the strength of BCR signaling (Paus et al. 2006). B cells with a highaffinity $\mathrm{BCR}$ undergo proliferation and plasmacytic differentiation into plasmablasts and finally short-lived plasma cells that secrete high amounts of IgM but non-somatically mutated antibodies (Jacob et al. 1993, Paus et al. 2006). B cells with an intermediate affinity BCR follow the second developmental path by migrating into the lymphoid follicles to form germinal centers (Thorbecke et al. 1994, Paus et al. 2006, Carter and Myers 2008). Here, B cells highly proliferate and undergo somatic hypermutation and isotype switching resulting in memory B cells and long-lived plasma cells that produce affinity-matured antibodies, mainly of the IgG isotype, some IgE and IgA (Coico et al. 1983, Ziegner et al. 1994). Memory B cells are released from the germinal centers and mount a quicker immune response upon re-exposure to their cognate antigen.

\subsubsection{Antigen-Presentation to T Cells}

Efficient antigen presentation is a prerequisite for the development of a T-cell-mediated immune response in vitro and in vivo. APCs are crucial for development of an immune response, since $\mathrm{T}$ cells are only activated when they recognize their specific antigen presented in combination with MHC and co-stimulatory molecules (Cassell and Schwartz 1994, Askew et al. 1995, Banchereau and Steinman 1998). Uptake of antigen by APCs, such as DCs, macrophages or B cells, takes place in the peripheral tissues or the lymphatic vessels. In the classical antigen presentation pathway, MHC class II molecules are assembled in the endoplasmatic reticulum (ER) and are associated with an invariant chain before the complex is transported to late endosomal compartments, called MIIC. In the endosomal compartments, MHC II molecules require HLA-DM (one of three polymorphic MHC II genes) to facilitate the exchange of the invariant chain with a specific peptide derived from a protein that was degraded in the endosomal pathway. MHC II molecules are then transported to the plasma membrane to present their peptide to $\mathrm{CD} 4^{+} \mathrm{T}$ cells (Neefjes et al. 
2011). In addition to endocytosis, B cells are able to take up antigen in a highly specific fashion via their B cell receptor (Abbas et al. 1985, Lanzavecchia 1985). In B cells, HLA-DM activity is restricted to acidic compartments, which is favorable for antigens internalized through the BCR (Alfonso et al. 1999).

Usually, presentation of antigens in the context of MHC class I molecules is restricted to endogenously derived peptides (Neefjes et al. 2011). Proteins of cytosolic and nuclear origin are degraded by proteasomes and translocated into the ER. There, MHC I molecules are assembled and loaded with the degraded antigens. The fully assembled peptide-MHC class I complexes leave the ER for presentation on the cell surface. The presentation of only intracellularly derived peptides in the context of MHC I molecules is however insufficient in the case of naïve $\mathrm{CD}^{+} \mathrm{T}$ cell priming, since they need to be activated by professional APCs before they can fulfill their cytotoxic effector function. Therefore, APCs also have the ability to load peptides derived from exogenous antigens to MHC class I molecules by a process called cross-presentation (Kurts et al. 2010). Cross-presentation and priming has since been shown to be essential for vaccination with protein antigens.

Encounter of T cells and activated APCs takes place in the secondary lymphoid organs, where organ structure brings antigen and APCs into close contact to T cells. In addition to TCRmediated signal induced by binding to a matching peptide-MHC II complex, activation of a $\mathrm{T}$ cells requires costimulatory signals. These are provided by the costimulatory surface molecules such as CD80 (B7-1) and CD86 (B7-2), which are highly upregulated on activated APCs (Lenschow et al. 1994, Bretscher 1999). Their receptor on the T cell surface is CD28. Absence of co-stimulatory signals at the time of TCR-MHC-antigen interaction results in anergy (Schwartz 2003).

DCs are known to be the most prominent APCs, since they play a critical role in inducing primary $\mathrm{T}$ cell responses, thereby leading to the establishment of immunological memory (Steinman 1991, Hart 1997, Banchereau and Palucka 2005, Palucka et al. 2011). Since the first report on DCs by Steinman and Cohn (Steinman and Cohn 1973) different subtypes arising from $\mathrm{CD}_{3} 4^{+}$bone marrow progenitors have been described (Shortman and Liu 2002, Steinman and Idoyaga 2010). DC progenitors develop in the bone marrow and migrate into peripheral tissues, where they encounter antigen. Upon antigen uptake and processing, DCs mature and migrate through the afferent lymphatics to the draining lymphoid organs, where 
they present antigen to $\mathrm{CD}^{+}$and $\mathrm{CD}^{+} \mathrm{T}$ cells. Moreover, $\mathrm{DCs}$ are able to induce a humoral immune response or activate cells of the innate immune system, such as NK cells, macrophages and eosinophils (Banchereau and Steinman 1998, Banchereau et al. 2000, Steinman 2008). Hence, DCs drive all arms of the immune system and therefore have extensively been studied for use as cellular adjuvant.

\subsection{Basic Principles of Cancer Immunology}

The immune system does not only protect us from invading pathogens, it also participates in the protection from cancer development by surveilling the body for transformed cells. A broad range of immune cell-types and immunological mechanisms are involved in this process.

\subsubsection{Cancer Immunosurveillance}

An immunological response against cancer has first been proposed by Paul Ehrlich in 1909 (Ehrlich 1909), who observed that mice were protected against rechallenge with aggressive tumor cells when previously vaccinated with slowly growing tumor cells. In 1954 Burnet and Thomas formulated the "cancer immunosurveillance hypothesis", which stated that innate and adaptive immune cells permanently monitor tissues for the presence of transformed cells and leading to their elimination (Burnet 1970, Burnet 1971, Thomas 1982). Due to a lack of direct experimental evidence this hypothesis remained highly controversial. It was not before the development of knock-out and immunodeficient mice that researchers were able to show that the immune system indeed plays a vital role in the control of neoplastic growth. These early studies identified some fundamental mechanisms of tumor immunosurveillance. First, the contribution of the cytokine IFN- $\gamma$ to protection against tumor formation (Kaplan et al. 1998, Street et al. 2001); second, the effect of perforin-mediated cytotoxicity of effector lymphocytes on tumor control (van den Broek et al. 1996, Street et al. 2001); and third, immune-deficient Rag1 and Rag2 knock-out mice, which are incapable of producing soluble antibodies or a functional TCR, were susceptible to tumor challenge (Shankaran et al. 2001). Further evidence for the existence of immune surveillance derived from observations in humans. Immunocompromised humans, like HIV patients or organ transplant recipients, are more susceptible to certain types of cancers than the general 
population (Grulich et al. 2007). Moreover, several bacterial infections were shown to be cancer-associated and several cancer-causing viruses have been identified (Mager 2006, Sarid and Gao 2011).

\subsubsection{Immunoediting}

The cancer immunosurveillance theory was extended from a simple host-protective role of the immune system to a more complex process, in which the immunological tumor microenvironment shapes tumor immunogenicity (Dunn et al. 2002, Schreiber et al. 2011, He et al. 2014). This process can lead to three possible outcomes. First, the innate and adaptive immune system recognizes and eliminates transformed cells (elimination phase). Second, in some cases tumor cells sustain elimination, due to tumor heterogeneity and selection pressure. This leads to an equilibrium state, in which tumor growth is controlled by the immune system (equilibrium phase). Third, tumor cells can become resistant to immune detection and enter the escape phase leading to the development of a malignant disease (escape phase).

\subsubsection{Tumor Evasion Strategies}

Immune-evasion strategies of tumor cells are manifold. They are the focus of interest for the development of a successful cancer immunotherapy. Active tolerance mechanisms induced by the tumor include downregulation of $\mathrm{MHC}$ molecules on the surface of tumor cells or the impairment of antigen processing. Tumor cells thereby evade recognition and destruction by cytotoxic T cells (Ferrone and Marincola 1995). Furthermore, tumor cells are able to shape the tumor microenvironment by release of suppressive anti-inflammatory cytokines, such as TGF- $\beta$ or IL-10 (Chen et al. 1994, Walker et al. 1998). More recently, tumor cells were shown to upregulate immune checkpoint proteins, like programmed cell death ligand 1 (PD-L1) or cytotoxic T-lymphocyte associated antigen 4 (CTLA-4). Thus, they further contribute to shaping the tumor microenvironment (Ramsay 2013). Passive tolerance mechanisms involve the recruitment of leukocytes into the tumor stroma. This includes mostly tumor-associated macrophages, mast cells, NK and NKT cells, T cells and B cells. The type of infiltration and the influence on survival outcome depends on tumor type and patient (Bremnes et al. 2011). However, regulatory $T$ and $B$ cells were shown to be recruited to the tumor site, negatively influencing the outcome for the patient (Whiteside 2008, Zhang et al. 2013). 
Overcoming evasion strategies of tumor cells and inducing an effective immune response by $\mathrm{T}$ cells is therefore the key to a successful treatment of cancer.

\subsection{Targeted Immunotherapy}

Given that cancer resists conventional surgery and nonsurgical treatment options and still remains the major cause of death worldwide, the development of novel prophylactic and therapeutic strategies is required. Impressive clinical success was achieved by immunotherapy in recent years (Couzin-Frankel 2013). In various studies, active and passive therapy approaches were shown to induce specific immune responses against various tumors with high therapeutic potential (Mellman et al. 2011).

\subsubsection{Tumor Antigens}

The immune system has to distinguish between normal and neoplastic tissue to protect the organism from malignant cells. This is often difficult, since proteins on cancer cells and normal tissue are similar in structure and their level of expression. However, there are certain tumor antigens, which are self derived, but trigger an immune response anyway. Over the last 20 years a large array of immunogenic tumor antigens has been identified and characterized. Two primary groups of antigens can be distinguished: Tumor-specific antigens and tumor-associated antigens.

\subsubsection{Tumor-Specific Antigens}

Tumor-specific antigens are unique to the tumor tissue. They arise from genetic alterations like point mutations, deletions or chromosomal translocation (Bielas et al. 2006) and may be associated with tumor formation. However, their usefulness for standard immunotherapy is limited, since they are highly patient-specific and manufacturing such a vaccine would be costly (Sensi and Anichini 2006). Some shared tumor-specific antigens have been identified, among them the oncogene ras and the mutated tumor suppressive gene p53 (Bos 1989, Chiba et al. 1990). 


\subsubsection{Tumor-Associated Antigens}

Tumor-associated antigens are shared by normal and neoplastic tissue, but their level or location of expression is differs. Tumor-associated antigens can be divided into three subgroups. Some of these antigens are usually expressed exclusively in germ cells of the testes. In cancer cells, those germ line genes can be aberrantly activated (Caballero and Chen 2009). Since germ cells do not express MHC molecules and thereby do not present antigen to T cells, such antigens can be considered as tumor-specific. One example is the melanoma-antigen family (MAGE) of proteins. The second subgroup represents antigens that are expressed during embryogenesis or particular stages of differentiation. Their use as target for cancer immunotherapy is limited since it may lead to autoimmune reactions. Some well studied examples are Melan-A, gp100 and tyrosine, which are expressed in healthy melanocytes, but also melanoma cells. Other examples are prostate-specific antigen (PSA) in prostate carcinoma or cell adhesion molecule (CEA) in colorectal carcinoma (Malati 2007). The third group is characterized by antigens that are overexpressed in tumor tissue, but are expressed in very low levels in normal tissue. These overexpressions are often the result of gene amplifications and are associated with initial tumor formation or growth. Only a small amount of these antigens, which include for example HER2 or telomerase, is required to elicit a $\mathrm{T}$ cell response and therefore the risk for autoimmunity is low (Disis and Cheever 1997).

\subsubsection{Passive Immunotherapy}

Exploiting the specificity of the immune system against cancer cells provides a promising therapeutic approach against cancer diseases with low risk for toxicity and side effects. Breaking self-tolerance and overcoming tumor-escape mechanisms as well as identifying the appropriate target and delivery platform are the key to a successful immunotherapy.

Passive immunotherapy with monoclonal antibodies today is a well established treatment modality. The number of clinically used monoclonal antibodies and their cancer-associated target proteins has increased dramatically over the past years. They act by antagonizing oncogenic signaling pathways, blocking tumor cells or initiating cell death. Great clinical efficacy has been reported for agents inhibiting immunological checkpoints. In 2012, Toplian et al. reported a study with 300 melanoma, kidney or lung cancer patients that were treated 
with anti-PD1 antibodies. One third of these patients showed a clinical response (Topalian et al. 2012). In 2013, the combined therapy with Ipilimumab (anti-CTLA-4) and Nivolumab (antiPD-L1) resulted in tumor reduction in one third of patients with metastasized, pretreated melanomas (Wolchok et al. 2013). One of the most recent examples of a successful antibody-based immunotherapy is the use of Brentuximab for targeting CD30 in patients with CD30-positive lymphomas (Theurich et al. 2013, Theurich et al. 2013, Theurich et al. in press).

\subsubsection{Active Immunotherapy}

In contrast to passive immunotherapy with monoclonal antibodies, active immunotherapy aims to induce a specific anti-tumor response by vaccination (Schlosser et al. 2014). Strategies include non-cellular vaccines with purified tumor-antigen, synthetic peptides or tumor lysates, and cellular vaccines. Moreover, ex vivo expanded and/or manipulated immune cells can be adoptively transferred into cancer patients. One promising approach are genetically engineered T cells expressing tumor-specific "chimeric antigen receptors" (CARs) to directly target tumor cells with high specificity. In 2013, two groups reported of 75 patients with leukemia, of which 45 patients were successfully treated with CARs (CouzinFrankel, 2013). Prophylactic cancer vaccines, which exploit the immunological properties of APCs to induce tumor-specific T cells, are already successfully used in the treatment of virally-induced cancer diseases (Kanwar et al. 2011). Therapeutically used vaccines showed first clinical success within the past years, including the first US Food and Drug Administration (FDA)-approved cell based immunotherapy on the basis of autologous DCs for the treatment of prostate carcinoma patients (Small et al. 2000). Strategies with ACPs loaded with tumor-associated antigens aim at inducing effective cellular and antibodymediated responses specific for antigens expressed by tumors, thereby avoiding unwanted side effects as seen by conventional approaches (Grabbe et al. 1995, Ward et al. 2002, Banchereau and Palucka 2005). The challenges remain in identifying immunogenic tumor antigens, overcoming tolerance and generating professional APCs that effectively and specifically induce T cells immunity. 


\subsubsection{Dendritic Cell Vaccination}

Cellular-based adjuvants are the object of intensive studies since- in contrast to non-cellular approaches- they reflect the physiological induction of a T cell response. A number of different subtypes of dendritic cells have been identified, since their first description by Steinman et al. and have been explored for their use as vaccine against cancer and infectious diseases (Steinman and Cohn 2007).

In mice, vaccination with bone-marrow derived ex vivo matured antigen-loaded DCs has been shown to induce protective and therapeutic anti-tumor immunity (Mayordomo et al. 1995, Parkhurst et al. 1996, Zitvogel et al. 1996). Several strategies to deliver antigen to DCs have been exploited: DCs are simply loaded with peptides or proteins (Parkhurst et al. 1996, Li et al. 2002), they are transfected with mRNA or DNA plasmids (Boczkowski et al. 1996) or transduced with viral vectors (Ribas et al. 2002). Other strategies include tumor-DC fusions, loading DC with dying tumor cells (Albert et al. 1998, Berard et al. 2000) or direct target of DC surface molecules like DEC205 or DC-SIGN to facilitate antigen uptake (Gilboa 1999, Fong and Engleman 2000).

The potential of this approach in a human setting could be demonstrated in numerous studies. In particular, studies with healthy volunteers demonstrated that a single vaccination dose of DCs is sufficient to induce an antigen-specific T cell response (Dhodapkar et al. 1999). However, after more than 200 clinical studies with DC-based vaccines, the responserate to vaccination has been disappointingly low (Draube et al. 2011). Therefore, in order to achieve reliable responses, several questions need to be addressed before this approach can be routinely applied in the clinic. These include the identification of the appropriate DC subtype, the optimal activation status, the injection route, the injection interval or dose.

DCs cannot be generated in large quantities or in sufficient purity, thereby making their development for the clinical application laborious and expensive. In most studies the purity of the applied DCs is not determined at all or it does not reach more than $80 \%$ (Draube et al. 2011). An insufficient purity makes it difficult to distinguish between the effect of the DCs itself and the effect of the bystander cells (Figdor et al. 2004, Rosenberg 2004, Schultze et al. 2004). In addition, the optimal maturation status of the generated DCs is of critical importance. Immature DCs are usually differentiated ex vivo from $\mathrm{CD}_{3} 4^{+}$progenitors by treatment with granulocyte/macrophage colony-stimulating factor (GM-CSF) and IL-4. 
However, antigen-presentation by immature DC subsets results in undesired antigen-specific tolerance mechanisms (Mahnke et al. 2002). Maturation or terminal differentiation of DCs can be achieved by antagonizing their toll-like receptors with CpG-ODN (Caux et al. 1992) or triggering tumor-necrosis factor (TNF) receptor signaling with LPS or CD40 ligation (Romani et al. 1994, Sallusto and Lanzavecchia 1994). However, the stimulus used for maturation results in the release of different cytokine subsets and therefore in different $\mathrm{T}$ cell responses, either of tolerogenic or immunogenic nature (Steinman et al. 2003, Mailliard et al. 2004, Liu et al. 2009).

Another critical point for the use of DCs in a clinical setting is their lack of CD62 ligand expression, a key molecules for migration through the high endothelial venues, and their resulting failure of lymph node homing (von Andrian and Mempel 2003). Therefore, the optimal injection route does not only influence $\mathrm{DC}$ homing and $\mathrm{T}$ cell response, but also determines the clinical outcome. Injection routes with easy access to the draining lymph nodes were shown to result in an improved clinical response than for example intravenous injections (Draube et al. 2011). Although less than $4 \%$ of the injected DCs reach the draining lymph nodes, intradermal or intravenous injections are preferred in clinical studies, since intralymphatic and intranodal injections remain challenging (Ridolfi et al. 2004, Quillien et al. 2005).

The first autologous DC-based therapeutic cancer vaccine, Sipuleucel-T (PROVENGE), has been approved by the FDA for the treatment of prostate cancer in 2010 (Cheever and Higano 2011). This therapy is based on ex vivo generated DCs that target prostate acid phosphatase (PAP) antigen on prostate cancer cells and prolongs the median survival of men with advanced castrate-resistant prostate cancer by 4.1 month. However, clinical studies on Sipuleucel-T have been criticized afterwards and, taking gain of quality-adjusted life years into account, the treatment with Sipuleucel-T has been evaluated to be cost-ineffective (Graff and Chamberlain 2015).

Taken together, combining immunomodulatory agents and DCs with conventional treatment like radiation and chemotherapy currently provide the most promising approaches to improve therapeutic efficacy of vaccination against cancer (Le et al. 2010, Palucka et al. 2010). 


\subsection{B Cells as Antigen-Presenting Cells}

Even though B cells are less efficient than DCs at capturing antigens by pinocytosis, FcReceptor (FcR)-mediated uptake or adsorption (Chesnut et al. 1982, Grey et al. 1982, Batista and Neuberger 1998, Rodriguez-Pinto 2005), they are increasingly recognized to play a crucial role in the initiation of $T$ cell responses. However, to fully extend their function as APCs, B cells require two activation signals. The first one is the crosslinking of the BCR by its antigen, which induces growth, proliferation and survival of the B cell. Moreover, it leads to expression of the costimulatory molecule CD86 (Lenschow et al. 1994). The second signal results from the interaction with $\mathrm{CD}^{+} \mathrm{T}$ cells and is mediated by CD40-CD40 ligand (CD40L) interaction and the secretion of IL-4 by the T cells (Ranheim and Kipps 1993, Evans et al. 2000). CD40-derived signals induce B-cell activation and promote several changes related to their antigen-presenting function: CD40-activated B cells (CD40B cells) upregulate the expression of MHC class I and II and costimulatory molecules CD80 and CD86, enhance antigen processing and are able to activate naïve T cells (Kennedy et al. 1994, Faassen et al. 1995).

\subsubsection{CD40-CD40L Interaction}

CD40-CD40L interaction exerts profound effects on DCs, B cells, endothelial cells and many other cells of the hematopoietic and non- hematopoietic compartments (Elgueta et al. 2009). The CD40 receptor and its ligand are both transmembrane proteins of the TNF superfamily (van Kooten and Banchereau 2000). The CD40L is primarily expressed on T cells, but also on activated B cells, platelets and under inflammatory conditions on other cells of the innate and adaptive immune system (Carbone et al. 1997). The soluble form of the CD40L was shown to express similar activities as its transmembrane form (Graf et al. 1995, Mazzei et al. 1995). In B cells, CD40 signaling promotes germinal center formation, immunoglobulin (Ig) isotype switching, somatic hypermutation of the Ig to enhance affinity for antigen and the formation of long-lived plasma cells and memory B cells (Danese et al. 2004, Elgueta et al. 2009). 


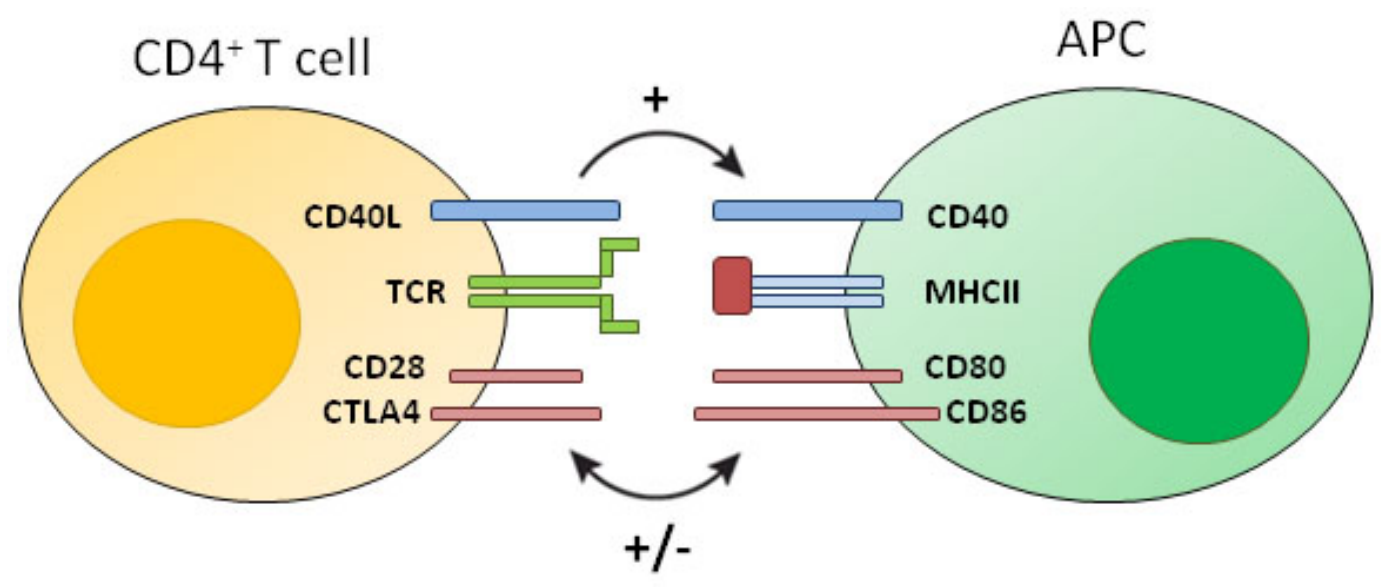

Fig. 1.2 APC activation upon encounter with an antigen-specific $\mathrm{CD}^{+} \mathrm{T}$ cell. Interactions that control the outcome of $\mathrm{CD}^{+} \mathrm{T}$ cell-APC encounter are indicated by the receptor-ligand pairs. CD40L, CD40 ligand; TCR, T-cell antigen receptor; CTLA4, cytotoxic T lymphocyte-associated protein 4; MHCII, major histocompatibility complex type II protein (Haanen and Schumacher 2007).

More importantly, CD40 engagement promotes B cell cytokine production, the expression of costimulatory molecules on their surface and facilitates cross-presentation of antigen (Robson et al. 2008, de Wit et al. 2010). Furthermore, CD40 signaling supports survival of germinal center B cells, DCs and endothelial cells (Bishop et al. 2007) and its deregulation has been observed to play a role in multiple autoimmunity diseases (Peters et al. 2009).

The importance of this signaling pathway in acquired immune responses makes it a promising target for pharmacological manipulations for the treatment of immunological diseases. Interference of the CD40-CD40L interaction by use of blocking CD40-antibodies showed positive effects on uncontrolled immune reactions like inflammation, autoimmunity or allo-graft rejection (Larsen et al. 1996, Kirk et al. 1997, Kirk et al. 2001, Pearson et al. 2002, Grammer et al. 2003, Sidiropoulos and Boumpas 2004). Moreover, enhancement of the CD40-CD40L interaction by use of antagonistic CD40-antibodies was shown to result in anti-proliferative anti-tumor effects or meditation of immune response against tumors (Mackey et al. 1998, Diehl et al. 1999, Tong and Stone 2003, Watanabe et al. 2003, Eliopoulos and Young 2004, Vonderheide et al. 2007). Because of their potential as APC stimulators, CD40 agonists have been selected as one of the twelve immunotherapy drugs that could cure cancer (Cheever 2008). 


\subsubsection{CD40-Activated B Cells}

In 1991, Banchereau et al. discovered that long-term human B-cell lines could be established by stimulation with the CD40L and IL-4 (Banchereau et al. 1991, Banchereau and Rousset 1991). Since then several strategies have been developed to manipulate the CD40-CD40L interaction to activate B cells (Neron et al. 2011). Schultze et al. established a culture system, in which human B cells can be activated and expanded by stimulation with the CD4OL and IL-4 (Schultze et al. 1997). Under constant cultivation on CD40L expressing feeder cells, human and murine primary B cells can be expanded from small amount of peripheral blood, upregulate costimulatory, $\mathrm{MHC}$ and adhesion molecules and show an increased antigen presenting capacity in vitro (Liebig et al. 2009, Liebig et al. 2010). The expression level of MHC and costimulatory molecules is comparable to those of CD4OL/IFN- $\gamma$ or TNF- $\alpha$ matured DCs (Wiesner et al. 2008). In vitro these CD40-activated B cells present antigen in an MHC class I or II- restricted manner to induce naïve or memory $\mathrm{CD} 8^{+}$and $\mathrm{CD} 4^{+} \mathrm{T}$ cells responses, respectively (Schultze et al. 1997, von Bergwelt-Baildon et al. 2002, Lapointe et al. 2003, von Bergwelt-Baildon et al. 2004, Theurich et al. 2011) and overcome T cell tolerance as shown by generating autologous tumor antigen-specific $T$ cells in vitro by help of peptide-pulsed CD40B cells (von Bergwelt-Baildon et al. 2002, Shen et al. 2007, Wu et al. 2010). Moreover, CD40B cells have the capacity to attract $T$ cells and to home to secondary lymphoid organs (von Bergwelt-Baildon et al. 2006, Guo et al. 2009), which is essential for T cell-APC encounter and the induction of immunity. Human and murine CD40B cells express the respective factors and receptors crucial for homing to secondary lymphoid organs including CD62L, C-C-chemokine receptor (CCR) 7/ CXC-Motiv-Chemokinrezeptor (CXCR) 4, and leukocyte function antigen (von Bergwelt-Baildon et al. 2006, Klein-Gonzalez et al. accepted 2015). The receptors CCR7 and CXCR4 are functional and induce chemotaxis toward increasing chemokine concentrations of their ligands CCL21 and CXCL12, respectively (Fig. $1.3)$. 


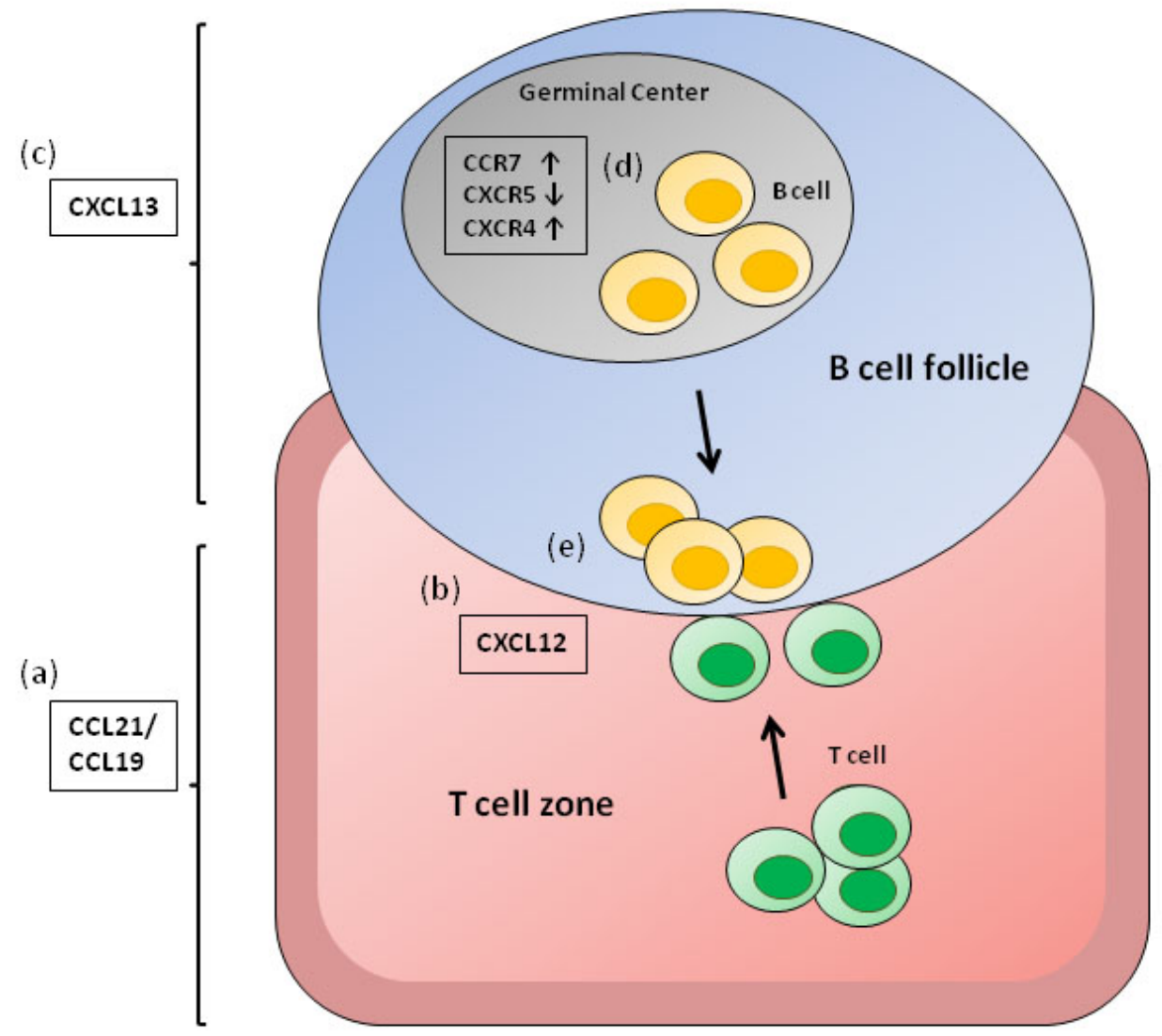

Fig. 1.3. Chemokines involved in lymphocyte positioning in the secondary lymphoid organs.

(a) CCL21/ CCL19, chemokines expressed in the T cell zones of secondary lymphoid organs and the ligand for CCR7; (b) CXCL12, the ligand for CXCR4 mostly involved in T cell zone entry; (c) and CXCL13, a chemokine made in B cell zones of lymphoid organs and the ligand for CXCR5. (d) The chemokine expression pattern of $\mathrm{CD} 40 \mathrm{~B}$ cells (e) allows them to migrate towards the $\mathrm{B} / \mathrm{T}$ cell boundary (KleinGonzalez et al. accepted 2015).

Nevertheless, very few studies have focused on the in vivo antigen-presenting capacity of CD40B cells and their contribution to anti-tumor immunity (Wennhold et al. 2013). In two preventive vaccination studies, tumor growth was delayed in mice that were immunized with CD40 B cells (Ritchie et al. 2004, Liebig et al. 2011). The group of Mason reported the first study in out-bred large animals using tumor RNA-loaded CD40B cells as cellular adjuvant in privately owned dogs with NHL (Sorenmo et al. 2011). However, the correlation between the immunological response and the clinical outcome was not significant.

Taken together, CD40B cells cannot only serve to identify tumor-antigens or to generate T cells for adoptive immunotherapy, but show also promise as a versatile platform for cellular cancer vaccines (Fujiwara et al. 2005, Ivanov et al. 2005, Kondo et al. 2009). Most of all, the results from preclinical studies of application as antigen presenting cells in 
preventive and therapeutic vaccination appears promising (von Bergwelt-Baildon et al. 2002, Coughlin et al. 2004, Van den Bosch et al. 2005, Yoon et al. 2005, Shen et al. 2008, Wu et al. 2010).

\subsubsection{Antigen-Specific B Cells}

Antigen-processing and -presentation, and thereby T cells activation, is $1000-10,000$ fold more effective in antigen-specific B cells compared to resting or lipopolysaccharide (LPS)activated polyclonal B cells (Abbas et al. 1985, Lanzavecchia 1990). The BCR has a high affinity for a given antigen and allows $B$ cells to concentrate very small quantities of their specific antigen and present the antigen efficiently. In contrast, presentation after uptake by fluid phase pinocytosis in B cells requires about 5000 higher concentrations.

Specific antigen uptake via the $B C R$ leads to three important changes in the antigen processing machinery that facilitates the traffic of antigen and MHC class II molecules and the generation of peptide-MHCIl complexes (Rodriguez-Pinto 2005). First, BCR receptor ligation induces its internalization and the traffic towards $\mathrm{MHCll}$ rich compartments (MIIC), the site of peptide-MHCII complex formation. Second, BCR signaling upregulates $\mathrm{MHCI}$ expression and trafficking through the MIIC. Finally, BCR crosslinking induces changes in the MIIC to generate the adequate environment for loading of $\mathrm{MHCll}$ with peptides. A second characteristic of antigen processing in B cells differs from that in other antigen presenting cells. The expression of HLA-DO, a non-classical MHCII molecule that is expressed only in B cells and thymic epithelium, modulates peptide loading of MHCII molecules by HLA-DM and favoring presentation of peptides derived from antigens internalized through the BCR (Alfonso et al. 1999, Denzin et al. 2005).

\subsection{Purpose of this Study}

Although polyclonal $\mathrm{CD} 40 \mathrm{~B}$ cells were shown to efficiently induce anti-tumor immunity in vivo, the tumor control is still suboptimal when using polyclonal CD40B cells as cellular adjuvant (Liebig et al. 2011). Based on the fact that antigen-specific B cells present antigen very efficiently when the antigen concentration is low, this study aimed at investigating whether the use of tumorantigen-specific CD4OB cells instead of polyclonal CD40B cells improves their antigen-presenting function. For this purpose polyclonal and antigen-specific 
CD40B cells were compared with regard to their potential to induce antigen-specific $\mathrm{T}$ cell reactions in vitro and in vivo. Moreover, the migratory behavior of tumorantigen-specific CD40B cells in healthy and tumor bearing mice was investigated with the purpose of exploiting their potential as drug delivery and imaging vehicle. With regards to the use of antigen-specific B cells as cellular adjuvant for cancer immunotherapy, their antigenpresenting function and antibody-secreting capacity were combined for immunotherapy in tumor bearing mice. 


\section{Material and Methods}





\section{Material and Methods}

\subsection{Material}

\subsubsection{Chemical}

For preparation of buffers and solutions, chemicals of analytical purity were used exclusively.

Bovine Serum Albumin (BSA)

CFSE

Active hepatitis $b$ virus Hepatitis $B$

Surface Antigen full length protein (HBV-antigen)

Albumin from Chicken Egg White (OVA)

Dimethyl sulfoxide (DMSO)

D-Luciferin

Ethanol

Ethylenediaminetetraacetic acid (EDTA)

Hygromycin B

Isofluran

Ovalbumin peptide (aa 257-264)

Paraformaldehyde (PFA)

Sodium hydrogen carbonate $\left(\mathrm{NaHCO}_{3}\right)$

Sodium carbonate $\left(\mathrm{Na}_{2} \mathrm{CO}_{3}\right)$

Sodium chloride $(\mathrm{NaCl})$

Trypsin-EDTA

Tween-20

Sodium Dodecyl Sulfate (SDS)

\subsubsection{Consumables}

$5 \mathrm{ml} \mathrm{FACS}$ tubes

6-well culture dish

12-well culture dish

$15 \mathrm{ml}$ Falcon tube

$26 G 1 / 2$ needle

$50 \mathrm{ml}$ Falcon tube

$75 \mathrm{~cm}^{2}$ culture flask (adherent)

$75 \mathrm{~cm}^{2}$ culture flask (suspension)

96-well culture dish, round bottom

96-well high binding plate

$100 \mu \mathrm{m}$ cell strainer

Cryo Tubes

Glass object slides

MS Columns

LD Columns

LS Columns

Slide-A-Slizer G2 Dialysis Cassette
Sigma Adrich

Invitrogen

Abcam

Sigma Aldrich

Sigma Aldrich

Regis Technologies

Th Geyer

Sigma Aldrich

Sigma Aldrich

Actavis

Thermo Scientific

Sigma Aldrich

Sigma Aldrich

Sigma Aldrich

Sigma Aldrich

Life Technologies

Sigma Aldrich

Sigma Aldrich

Sarstedt

Sarstedt

Sarstedt

Sarstedt

Beckton Dickinson

Sarstedt

Sarstedt

Sarstedt

Sarstedt

Greiner

Greiner

Sarstedt

Sarstedt

Miltenyi Biotech

Miltenyi Biotech

Miltenyi Biotech

Thermo Scientific 


\title{
2.1.3 Kits
}

EasySep Mouse T Cell Enrichment Kit EasySep Mouse Biotin Selection Kit

Pierce Biotin Quantitation Kit

\subsubsection{Devices}

EOOS 350D digital camera

Gallios Flow Cytometer

HeraCell 150i CO2 Incubator

Heraeus Megafuge 16R

HeraSafe hood

Microplate Reader $\mu$ Quant

Neubauer Counting Chamber

Silk-epil FG1100

Televal 31 microscope

Vernier Caliper

Water bath WNB14 Set

Xenogen IVIS 200
Stem Cell Technologies

Stem Cell Technologies

Thermo Scientific

\author{
Canon \\ Beckman Coulter \\ Thermo Scientific \\ Thermo Scientific \\ ThermoScientific \\ BIO-TEK Instruments \\ OptikLabor \\ Braun \\ Zeiss \\ Hogotex \\ Memmert \\ Perkin Elmer
}

\subsubsection{Cell Culture}

If not described differently, pipette tips were obtained from BD Falcon.

\subsubsection{Cell Culture Reagents}

$10 \times$ Phosphate buffered saline (PBS)

AB-Human serum

AIM-V medium

Anti-mouse CD40Ligand (CD40L) antibody

Anti-mouse IgM(mu-chain), unconjugated

$\beta$-Mercaptoethanol ( $\beta$-ME)

CpG ODN 2395

Cyclosporin A

DMEM medium

Fetal Bovine Serum (FBS)

G418

GM-CSF

HEPES

IMDM medium

Incomplete Freund's Adjuvant

LPS from Escherichia coli 055:B5

MEM

Penicillin/ Streptomycin

rh Insulin

rh Interleukin-4

rh Transferrin
Life Technologies

Biochrom

Life Technologies

Novus Biologicals

Sigma Aldrich

Sigma Adrich

Miltenyi Biotech

Sigma Adrich

Life Technologies

Lonza

Biochrom

Immunotools

Life Technologies

Life Technologies

Sigma Aldrich

Sigma Aldrich

Life Technologies

Life Technologies

Novo Nordisk

Immunotools

Sigma Aldrich 
rm Interleukin-4

rm Interleukin-21

RPMI 1640 medium

Trypan Blue Stain 0.4 \%

VLE-RPMI medium

\subsubsection{Media}

DC medium

Freezing medium

HeLa standard medium

HeLa selection medium

Human CD40B medium

Murine CD40B medium

NIH standard medium

Tumor medium
Immunotools

Immunotools

Life Technologies

Life Technologies

Biochrom

VLE-RPMI medium

$+5 \%$ FBS

$+50 \mu \mathrm{M} \beta-\mathrm{ME}$

$+10 \%$ Pen/Strep

FBS $+10 \%$ DMSO

RPMI 1640

$+300 \mu \mathrm{g} / \mathrm{ml} \mathrm{L-Glutamine}$

$+10 \%$ FBS

$+10 \mathrm{mM}$ HEPES

$+10 \%$ Pen/Strep

RPMI 1640

$+300 \mu \mathrm{g} / \mathrm{ml}$ L-Glutamine

$+10 \%$ FBS

$+10 \mathrm{mM}$ HEPES

$+10 \%$ Pen/Strep

+ $0.2 \mathrm{mg} / \mathrm{ml}$ Hygromycin B

IMDM

$+584 \mu \mathrm{g} / \mathrm{ml} \mathrm{L-Glutamine}$

+25 mM HEPES

$+10 \%$ AB-Human serum

$+50 \mu \mathrm{g} / \mathrm{ml}$ rh Transferrin

$+5 \mu \mathrm{g} / \mathrm{ml}$ rh Inuslin

$+10 \%$ Pen/Strep

DMEM

$+580 \mu \mathrm{g} / \mathrm{ml}$ L-Glutamine

$+4.5 \mathrm{mg} / \mathrm{ml}$ Glucose

$+10 \% \mathrm{FBS}$

+10 mM HEPES

$+0.1 \mathrm{mM}$ MEM

$+10 \%$ Pen/Strep

DMEM-Ham's/ F12

$+365 \mu \mathrm{g} / \mathrm{ml}$ L-Glutamine

$+10 \%$ FBS

+10 mM HEPES

$+10 \%$ Pen/Strep

DMEM

+ $580 \mu \mathrm{g} / \mathrm{ml}$ L-Glutamine

$+10 \%$ FBS

$+10 \mathrm{mM}$ HEPES

$+10 \%$ Pen/Strep 


\subsubsection{Reagents}

ABTS Peroxidase Substrate

Biotin anti-mouse IgG1

CD19 MicroBeads human

CD19 MicroBeads mouse

Cell wash

EZ-Link NHS-Biotin Reagent

HRP Avidin

Pancoll Human (density $1.077 \mathrm{~g} / \mathrm{l}$ )

Pancoll Mouse (density $1.086 \mathrm{~g} / \mathrm{l}$ )

Purified anti-chicken Ovalbumin

\subsubsection{Solutions and Buffers}

1x PBS

Blocking solution

Coating buffer

EasySep medium

MACS buffer

PBS/Tween

\subsubsection{Software}

FlowJo Software

GraphPad Prism

Kaluza Software

Living Image Software
KPL

Biolegend

Miltenyi Biotech

Miltenyi Biotech

Beckton Dickinson

Thermo Scientific

Biolegend

PAN Biotech

PAN Biotech

Biolegend
1 L 10x PBS, 9 L Ampuwa 1x PBS, $10 \%$ FBS

$8.4 \mathrm{~g} \mathrm{NaHCO}_{3}, 3.56 \mathrm{~g} \mathrm{Na}_{2} \mathrm{CO}_{3}$, add $\mathrm{H}_{2} \mathrm{O}$ up to $1.0 \mathrm{~L}$, $\mathrm{pH}$ to 9.5

1x PBS, $2 \%$ FBS, 1 mM EDTA

1x PBA, $0.5 \%$ BSA, 2 mM EDTA

$1 \mathrm{~L} 1 \mathrm{x}$ PBS, $0.5 \mathrm{ml}$ Tween-20

\subsection{Methods}

\subsubsection{Cell Subset Enrichment}

\subsubsection{Purification of Murine Lymphocytes from Spleen}

Spleens were removed from 7-12 week old mice. A single suspension was prepared by squeezing the tissue through a $100 \mu \mathrm{m}$ cell strainer. For purification of murine lymphocytes density-gradient centrifugation was performed. Cells of two spleens were resuspended in 8 $\mathrm{ml}$ murine $\mathrm{CD} 40 \mathrm{~B}$ medium and added onto a layer of $5 \mathrm{ml}$ mouse Pancoll separation medium. Cells were centrifuged at $1080 \times \mathrm{g}$ for $15 \mathrm{~min}$ without break. Afterwards, the interphase, containing the splenocytes (lymphocytes from the spleen), was carefully collected with a $20 \mathrm{G}$ needle and cells were washed with $10 \mathrm{ml} \mathrm{PBS}$. 


\subsubsection{Purification of Murine Lymphocytes from Lymph Nodes}

Single cell suspensions from mesenteric and inguinal lymph nodes of 7-12 week old mice were prepared by squeezing the organs through a $100 \mu \mathrm{m}$ cell strainer into a culture dish. Cells were collected by washing the culture dish with $8 \mathrm{ml}$ murine CD40B medium.

\subsubsection{Purification of Human Lymphocytes from Blood}

Buffy coat preparations were obtained from healthy donors at the blood bank of our institution. The donors gave their consent and all the experiments were approved by our institutional ethical board.

Human peripheral blood mononuclear cells (PBMCs) were isolated from buffy coats by density-gradient centrifugation. $15 \mathrm{ml}$ blood was mixed with $20 \mathrm{ml} 1 \times$ PBS and layered on top of $15 \mathrm{ml}$ human Pancoll separation medium. Cells were centrifuged at $1080 \mathrm{x}$ for 15 min without break. Afterwards, the interphase, containing the lymphocytes, was carefully collected and cells were washed with $10 \mathrm{ml}$ PBS.

\subsubsection{Purification of Murine $\mathrm{CD}^{+}{ }^{+}$Cells}

Murine $\mathrm{CD}^{+}{ }^{+}$cells were negatively enriched from splenocytes (chapter 2.2.1.1) of 7-12 week old OT-I or OT-II mice using EasySep Mouse T Cell Enrichment Kit (Stem Cell Technologies, Canada) according to manufacturer's protocol. Briefly, cells were resuspended in EasySep medium at a concentration of $1 \times 10^{8}$ cells $/ \mathrm{ml}$. Cells were incubated for $10 \mathrm{~min}$ at RT with $50 \mu \mathrm{l} / \mathrm{ml}$ of Normal Rat Serum and $50 \mu \mathrm{l} / \mathrm{ml}$ of EasySep Mouse T Cell Enrichment Cocktail and for additional 2.5 min with $75 \mu \mathrm{l} / \mathrm{ml}$ of EasySep Streptavidin RapidSpheres. Afterwards, the tube with the cells was placed into the EasySep magnet for $2.5 \mathrm{~min}$. After the incubation time, the desired cell fraction was poured off into a new tube by inverting the magnet. The desired cell suspension was washed with $10 \mathrm{ml}$ AIM-V medium. T cell purity was determined by FACS analysis for $\mathrm{CD}^{+}$versus $\mathrm{CD} 19^{+}$cells.

\subsubsection{Purification of $\mathrm{CD}_{34}{ }^{+}$Bone Marrow Progenitor Cells}

Murine $\mathrm{CD}_{3} 4^{+}$progenitor cells were purified from bone marrow of hind limbs of C57BL/6N mice by positive selection with EasySep Biotin Selection Kit. 
Hind limbs of mice were isolated by cutting above the hip joint. The foot pad was cut below the ankle joint and femur and lower leg were divided. Muscle tissue was removed and bones put onto ice until further use. Bone tips were cut off and the bone marrow was washed from each side with a $26 \mathrm{G} 1 / 2$ needle by thorough rinsing with $4 \mathrm{ml}$ cold EasySep buffer into a falcon tube containing a buffer reservoir. The resulting cell suspension was washed through a $100 \mu \mathrm{m}$ cell strainer and centrifuged at $270 \times \mathrm{g}$ for $5 \mathrm{~min}$. The cells were resuspended in EasySep medium at a concentration of $1 \times 10^{8}$ cells $/ \mathrm{ml}$ and incubated with $10 \mu \mathrm{l} / \mathrm{ml}$ EasySep FcR-Block and $15 \mu \mathrm{l} / \mathrm{ml}$ anti-murine CD34-biotinylated antibody $(0.5 \mathrm{mg} / \mathrm{ml})$ for $15 \mathrm{~min}$ at RT. Afterwards, the cells were washed, resuspended in EasySep Medium at concentration of $1 \mathrm{x}$ $10^{8} \mathrm{cells} / \mathrm{ml}$ and incubated with $100 \mu \mathrm{l} / \mathrm{ml}$ EasySep Biotin Selection Cocktail for $15 \mathrm{~min}$ at RT. Afterwards, $50 \mu \mathrm{l} / \mathrm{ml}$ EasySep magnetic nanoparticles were added for additional $10 \mathrm{~min}$. The tube with the cells was placed into the EasySep magnet for 5 min. After the incubation time, the supernatant fraction was poured off by inverting the magnet. The desired cell fraction was collected by washing the tube 3 times with $1 \mathrm{ml} \mathrm{DC}$-medium.

\subsubsection{Purification of Murine and Human $\mathrm{CD}^{+} 9^{+} \mathrm{B}$ Cells}

Murine $C D 19^{+}$B cells were positively enriched from splenocytes (chapter 2.2.1.1) of 7-12 week old C57BL/6N or Luc ${ }^{+}$mice using murine CD19 MACS microbeads. Human $\mathrm{CD}_{19}{ }^{+} \mathrm{B}$ cells were positively enriched from PBMCs (chapter 2.2.1.3) using human CD19 MACS microbeads. Both isolations were performed according to manufacturer's protocols (Miltenyi Biotech, Germany) with minor modifications. Briefly, murine splenocytes or human PBMCs were resuspended in MACS buffer at a concentration of $17 \times 10^{7}$ cells $/ \mathrm{ml}$. Murine or human CD19 microbeads were added at a concentration of $127.5 \mu \mathrm{l} / \mathrm{ml}$ and cells were incubated for $15 \mathrm{~min}$ at $4^{\circ} \mathrm{C}$. Afterwards, cells were washed with $10 \mathrm{ml}$ MACS buffer and the pellet was resuspended in $5 \mathrm{ml}$ MACS buffer before the suspension was applied to the MACS LS column. After the column was washed two times with $3 \mathrm{ml}$ MACS buffer, the desired cell fraction was removed by firmly pushing the plunger into the column. Cells were washed with additional $10 \mathrm{ml}$ murine or human CD40B medium by centrifuging them at $270 \times \mathrm{g}$ for $5 \mathrm{~min}$. B cell purity was determined by FACS analysis for $\mathrm{CD} 19^{+} \mathrm{B} 220^{+}$cells. 


\subsubsection{Purification of Murine and Human Antigen-Specific B Cells}

Murine and human antigen-specific B cells were isolated from splenocytes of 7-12 week old immunized $\mathrm{C} 57 \mathrm{BL} / 6$ or $\mathrm{Luc}^{+}$mice or from blood (chapter 2.2.1.3) of vaccinated healthy donors. Antigen-specific $B$ cells were enriched by labeling them with antigen tetramers and subsequent positive selection using the EasySep Biotin Selection Kit.

\subsection{Generation of Protein-Biotin Conjugates for Antigen Tetramers}

The antigens were biotinylated by using the EZ-Link NHS-Biotin Reagent (Thermo Scientific, Rockford, USA) according to manufacturer's protocol. $135.5 \mu \mathrm{l}$ of a $10 \mu \mathrm{M}$ biotin solution were added to $1.5 \mathrm{ml}$ of a $2 \mathrm{mg} / \mathrm{ml}$ protein solution. The mixture was incubated on ice for two hours. To remove excess non-reacted biotin, the solution was applied to a Slide-A-Lyzer G2 Dialysis Cassette (Thermo Scientific, Rockford, USA) over night at $4{ }^{\circ} \mathrm{C}$ in $1 \mathrm{~L} 1$ x PBS with one complete change of the buffer after $2 \mathrm{~h}$.

OVA (albumin from chicken egg white) was purchased from Sigma Aldrich (St. Louis, USA) and HBV antigen (Active hepatitis b virus Hepatitis B Surface Antigen full length protein) was purchased from Abcam (Cambridge, UK).

\subsection{Quantification of Biotinylation}

Biotinylation of antigens was checked using the Pierce Biotin Quantitation Kit (Thermo Scientific, Rockford, USA) according to manufacturer's protocol. $100 \mu \mathrm{l}$ of HABA/Avidin Premix were added to $800 \mu \mathrm{l} 1 \mathrm{x}$ PBS and the absorbance of the solution at $500 \mathrm{~nm}$ was recorded as $A_{500} \mathrm{HABA}$ /avidin. $100 \mu \mathrm{l}$ of the antigen-biotin solution were added to the mixture and once the value of the absorbance at $500 \mathrm{~nm}$ was stable, it was recorded as A500 $\mathrm{HAB} /$ avidin/ biotin sample. The number of moles of biotin per mole of protein was calculated as follows according to manufacturer's protocol:

1. Calculation \# 1 is for the concentration of biotinylated protein in $\mathrm{mmol} / \mathrm{ml}$ (before any dilution for the assay procedure ( $\mathrm{MW}=$ molecular weight):

$$
\mathrm{Mmol} \text { protein per } \mathrm{ml}=\frac{\text { protein concentration }(\mathrm{mg} / \mathrm{ml})}{\mathrm{MW} \text { of protein }(\mathrm{mg} / \mathrm{mmol})}=\text { Calc \# } 1
$$

2. Calculation \# 2 is for the change in absorbance at $500 \mathrm{~nm}$ in a cuvette:

$$
\Delta A_{500}=\left(0.9 \times A_{500} H / A\right)-\left(A_{500} H / A / B\right)=\text { Calc \#2 }
$$


3. Calculation \# 3 is for the concentration of biotin in $\mathrm{mmol}$ per $\mathrm{ml}$ of reaction mixture:

$=\frac{\text { mmol biotin }}{\mathrm{ml} \text { reaction mixture }}=\frac{\Delta \mathrm{A} 500}{(34000 \times \mathrm{b})}=\frac{\mathrm{Calc} \# 2}{(34000 \times \mathrm{b})}=$ Calc \# 3

4. Calculation \# 4 is for the $\mathrm{mmol}$ of biotin per $\mathrm{mmol}$ of protein:

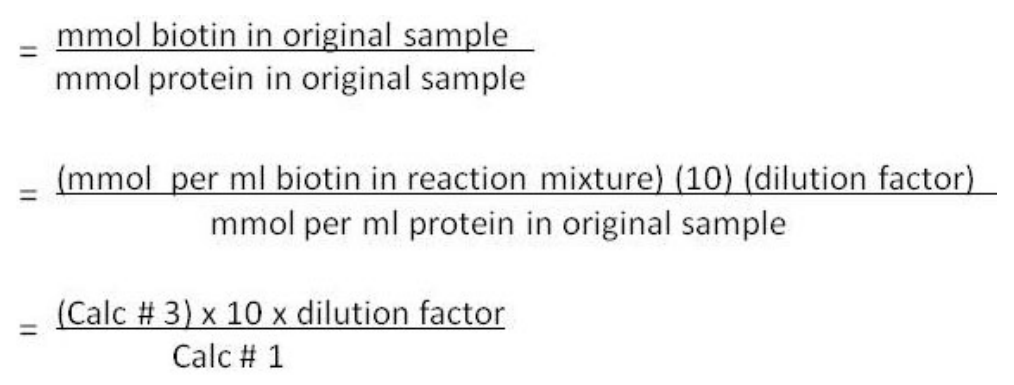

\subsection{Enrichment of Antigen-Specific B Cells}

OVA-specific B cells from splenocytes and HBV-specific B cells from blood were isolated using the EasySep Biotin Selection Kit. The cells were resuspended in EasySep medium at a concentration of $1 \times 10^{8}$ cells/ml and incubated with $10 \mu \mathrm{l} / \mathrm{ml}$ EasySep FcR-Block and $0.1-0.3$ $\mu \mathrm{g} / \mathrm{ml}$ (depending on the concentration of mmol biotin per mmol protein calculated in chapter 2.2.1.7.2) antigen-biotin conjugate for $10 \mathrm{~min}$ at RT. Afterwards, the cells incubated with $100 \mu \mathrm{l} / \mathrm{ml}$ EasySep Biotin Selection Cocktail and $4 \mu \mathrm{l} / \mathrm{ml}$ Streptavidin-PE for $15 \mathrm{~min}$ at RT and for another $10 \mathrm{~min}$ with $50 \mu \mathrm{l} / \mathrm{ml}$ EasySep magnetic nanoparticles. The tube with the cells was placed into the EasySep magnet for $5 \mathrm{~min}$. After the incubation time, the supernatant fraction was poured off by inverting the magnet. The cells were resuspended in $2.5 \mathrm{ml}$ EasySep medium. This procedure was repeated at least two times. The desired cell fraction was collected by washing the tube 3 times with $1 \mathrm{ml}$ murine or human CD4OB medium. The purity of murine or human antigen-specific B cells was determined by FACS analysis of antigen-specific $\mathrm{CD} 19^{+} \mathrm{B} 220^{+}$or $\mathrm{CD} 19^{+} \mathrm{CD} 20^{+}$cells, respectively.

\subsubsection{Purification of Murine Memory B Cells}

Murine memory B cells were enriched from splenocytes (chapter 2.2.1.1) of 7-12 week old C57BL/6N mice using murine Memory B Cell Isolation Kit (Miltenyi Biotech., Bergisch Gladbach, Germany). The isolation was performed according to manufacturer's protocol. Murine splenocytes were resuspended in MACS buffer at a concentration of $33 \times 10^{7}$ cells/ml. $100 \mu$ l of Memory B Cell Biotin-Antibody Cocktail, $50 \mathrm{ml}$ of Anti-IgG1-APC and $50 \mu \mathrm{l}$ 
of Anti-lgG2ab-APC were added per $10^{8}$ cells and incubated for 5 min at $4{ }^{\circ} \mathrm{C} .300 \mathrm{ml} \mathrm{MACS}$ buffer and $200 \mu \mathrm{l}$ of Anti-Biotin microbeads were added per $10^{8}$ cells for additional $10 \mathrm{~min}$. Afterwards, cells were applied to the MACS LD column. Unlabelled cells were collected and centrifuged at $270 \times \mathrm{g}$ for $5 \mathrm{~min}$. Cells were resuspended In $400 \mu \mathrm{l}$ MACS buffer and $100 \mu \mathrm{l}$ of Anti-APC microbeads were added for $15 \mathrm{~min}$ at $4{ }^{\circ} \mathrm{C}$. The cells were washed at $270 \mathrm{xg}$ for 5 min, resuspended in $500 \mu \mathrm{l}$ buffer and applied to the MACS MS column. After the column was washed two times with $500 \mu \mathrm{l}$ MACS buffer, the desired cell fraction was removed by firmly pushing the plunger into the column.

\subsubsection{Cell Culture}

If not described differently, all cell lines were incubated at $37{ }^{\circ} \mathrm{C}$ in a humidified atmosphere with $5 \% \mathrm{CO}_{2}$. Cell culture was performed at sterile conditions under a safety cabinet with sterile solutions, glass and plastic ware. All centrifugation steps in cell culture were performed in a Heraeus Megafuge 16R (Thermo Scientific).

\subsubsection{Counting of Cells}

For counting, an aliquot of the cell suspension was diluted with 10x with Trypan Blue Stain $0.4 \%$ and counted using a Neubauer Counting Chamber. $10 \mu \mathrm{l}$ of the cell suspension were applied to the counting chamber and bright cells within one big quadrant of the Neubauer chamber were counted as viable using a light optical microscope with 20 fold magnification. The average cell number of per quadrant was multiplied by the dilution factor and by the chamber factor $10^{4}$, resulting in the number of cells per $1 \mathrm{ml}$. The relative growth a cultures during a whole cultivation period was assessed by calculating the relative increase between two passages.

\subsubsection{Cryopreservation and Thawing of Cells}

For cryopreservation, cells were suspended in freezing medium, at a density of $3-10 \times 10^{6}$ cells $/ \mathrm{ml}$. The cell suspension was aliquoted into cryo tubes and slowly frozen at $-80{ }^{\circ} \mathrm{C}$ in a freezing chamber. $24 \mathrm{~h}$ later, tubes were transferred into liquid nitrogen for long-term storage. 
Frozen cells were rapidly thawed in a water bath at $37^{\circ} \mathrm{C}$ until a small ice clump was left in the tube. Cells were transferred to an excess of medium and centrifuged at $270 \times \mathrm{g}$ for $5 \mathrm{~min}$.

\subsubsection{Trypsinization of Adherent Cells}

The medium was removed and the adherent cells were washed with $10 \mathrm{ml}$ of $1 x$ PBS. After removing the PBS, $4 \mathrm{ml}$ of Trypsin-EDTA were added to a $75 \mathrm{~cm}^{2}$ flask. Cells were incubated at $37^{\circ} \mathrm{C}$ for 5-10 min. To stop trypsinization, $10 \mathrm{ml}$ of medium were added to the cells. Cells were harvested and centrifuged at $200 \mathrm{xg}$ for $5 \mathrm{~min}$ and resuspended in standard medium.

\subsubsection{Assessment of Morphology by Microscopy}

Representative sections of cultures were photographed by using an inverted phase Zeiss Televal 31 microscope fitted with a Canon EOOS 350D digital camera.

\subsubsection{Assessment of Morphology by Pappenheim Staining}

$10 \mu \mathrm{l}$ of a cell suspensions were placed at a density of $1 \times 10^{6}$ cells $/ \mathrm{ml}$ on a glass object slide. The cell suspension was allowed to dry over night. Pappenheim staining according to standard protocols were kindly performed in the Laboratory for Hematological Diagnostic in the Department for Internal Medicine I at the University Hospital Cologne, Germany. Pictures were taken using a Zeiss AxioPhot microscope at a magnification of $x 63$. Pictures were taken using dhs-Bilddatenbank.

\subsubsection{Cell Lines}

\subsubsection{Culture of the tmuCD40L HeLa Cell Line}

The tmuCD40L HeLa cell line, an adherent human epithelial cell line, was kindly provided by Clemens Wendtner (Klinikum Schwabing, Munich, Germany). Cell passaging was performed twice a week. Adherent tmuCD40L HeLa cells were trypsinized (chapter 2.2.1.3) and resuspended in $10 \mathrm{ml} \mathrm{HeLa} \mathrm{standard} \mathrm{medium} \mathrm{in} \mathrm{order} \mathrm{to} \mathrm{determine} \mathrm{cell} \mathrm{number} \mathrm{(chapter}$ 2.2.1.1). $2 \times 10^{6}$ cells in $10 \mathrm{ml}$ selection medium were seeded in a $75 \mathrm{~cm}^{2}$ culture flask and incubated at standard conditions. Stable expression of the CD40L was tested once a week by flow cytometry using a PE-conjugated anti-mouse CD154 (CD40L) antibody.

For generation of murine CD4OB cells, tmuCD40L HeLa cells were lethally irradiated 3 times with $26 \mathrm{~Gy}$ and subsequently plated on sterile 6-well culture plate at a density of $0.4 \times 10^{6}$ 
cells/well in $2 \mathrm{ml}$ HeLa standard medium. After 4-24 h of incubation at standard conditions, cells became adherent and were used for co-cultures with murine CD40B cells.

\subsection{Culture of the NIH3T3/tCD40L Cell Line}

The NIH3T3/tCD40L cell line, an adherent murine fibroblast cell line, was kindly provided by Gordon Freeman (DFCl, Boston, USA). Cell passaging was performed twice a week. Adherent NIH3T3/tCD40L cells were trypsinized (chapter 2.2.1.3) and resuspended in $10 \mathrm{ml} \mathrm{NIH}$ standard medium in order to determine cell number (chapter 2.2.1.1). $1.5 \times 10^{6}$ cells in $10 \mathrm{ml}$ $\mathrm{NIH}$ standard medium supplemented with G-418 $(0.7 \mathrm{mg} / \mathrm{ml})$ were seeded in a $75 \mathrm{~cm}^{2}$ culture flask and incubated at standard conditions. Stable expression of the CD40L was tested once a week by flow cytometry using a PE-conjugated anti-human CD154 (CD40L) antibody.

For generation of human CD4OB cells, NIH3T3/tCD4OL cells were lethally irradiated 2 times with $26 \mathrm{~Gy}$ and subsequently plated on sterile 6-well culture plate at a density of $0.2 \times 10^{6}$ cells/well in $2 \mathrm{ml} \mathrm{NIH}$ standard medium. After 4-24 h of incubation at standard conditions, cells became adherent and were used for co-cultures with human CD40B cells.

\subsection{Culture of the E.G7 Lymphoma Cell Lines}

The murine suspension lymphoma cell line EL4 and the OVA-expressing E.G7 cell line (EL4 background) were kindly provided by the Laboratory of Tomo Šarić (Department for Neurophysiology, University Hospital Cologne, Germany). Cell passaging was performed three times a week by harvesting the cell suspension. Cells were centrifuged at $270 \times \mathrm{g}$ for 5 min and resuspended in $10 \mathrm{ml}$ tumor medium for counting (chapter 2.2.1.1). Cells were culture at a concentration of $0.1 \times 10^{6}$ cells $/ \mathrm{ml}$ in a $75 \mathrm{~cm}^{2}$ culture flask and incubated at standard conditions. E.G7 cells were supplemented with G-418 $(1 \mathrm{mg} / \mathrm{ml})$.

\subsubsection{Generation of Murine and Human CD40B Cells}

Murine or human CD40B cell cultures were generated as described previously (Liebig et al. 2009, Liebig et al. 2010). In short, CD19+-purified (chapter 2.2.1.6) or antigen-enriched B cells (chapter 2.2.1.7) were resuspended at a concentration of $1.25 \times 10^{6} \mathrm{cell} / \mathrm{ml}$ in murine or human CD40B medium, respectively. The human medium was freshly supplemented with 1 $\mathrm{U} / \mathrm{ml}$ of IL-4 and $0.63 \mu \mathrm{g} / \mathrm{ml}$ cyclosporin A. The murine medium was additionally 
supplemented with $100 \mu \mathrm{M} \beta$-ME. For co-cultures, the supernatant of the previously prepared 6-well feeder cell plates (chapter 2.2.2.6.1 and 2.2.2.6.2, respectively) were removed and the plates were washed with $1 \times$ PBS. $4 \mathrm{ml}$ of the lymphocyte cell suspension were gently added to each well of the 6-well plate and incubated at standard conditions.

Feeder cells of CD40B cultures were refreshed twice a week. CD40B cells were harvested by vigorously pipetting the cell suspension up and down. The cells were washed at $270 \times \mathrm{g}$ for 5 min and resuspended at a concentration of $1.25 \times 10^{6}$ cells $/ \mathrm{ml}$ in murine or human CD40B medium. IL-4, $\beta-\mathrm{ME}$ and cyclosporin A were freshly supplemented at the concentrations mentioned above. The cell suspension was applied to fresh cultures of feeder cells and cultivated at standard conditions. The expression of co-stimulatory and MHC-molecules and surface immunoglobulins was determined twice a week by fluorescence-associated cell sorting (FACS) analyses.

\subsubsection{Generation of Murine Dendritic Cells}

For in vitro generation of murine dendritic cell cultures, $\mathrm{CD} 34^{+}$bone marrow progenitor cells (chapter 2.2.1.5) were used. The enriched cells were cultivated at a concentration of $0.25 \mathrm{x}$ $10^{6} \mathrm{cell} / \mathrm{ml}$ in murine DC medium supplemented with $500 \mathrm{U} / \mathrm{ml}$ of murine GM-CSF and 1 $\mathrm{U} / \mathrm{ml}$ murine IL-4. $2 \mathrm{ml}$ of the cell suspension was applied to each well of a 12-well plate and incubated at standard conditions. On days 3 , and 5 of the cultivation period, $1 \mathrm{ml}$ medium from each well was carefully removed and replaced by $1 \mathrm{ml} \mathrm{DC}$ medium supplemented with $1000 \mathrm{U} / \mathrm{ml}$ of murine GM-CSF and $2 \mathrm{U} / \mathrm{ml}$ of murine IL-4. For maturation of DCs, the medium was replaced as described above, supplemented with either $10 \mathrm{ng} / \mathrm{ml}$ LPS or $1 \mu \mathrm{g} / \mathrm{ml}$ antimouse CD40L antibody and cultivated over night at standard conditions. For further use, DCs were harvested by gently detaching them from the culture plate with a cell scraper. Purity of DC cultures was determined by FACS analyses of the $C D 11 c^{+} C D 11 b^{+}$cell population and checked for the expression of co-stimulatory molecules.

\subsubsection{Generation of Antibody-Secreting Plasma Cells}

For in vitro generation of antibody-secreting plasma cells, CD19+-purified (chapter 2.2.1.6) or antigen-enriched $B$ cells (chapter 2.2.1.7) were resuspended at a concentration of $1 \times 10^{6}$ cells/ml in CD40B medium. The medium was supplemented with different stimuli, including IL-4 (1 U/ml), IL-21 (50 ng/ml), anti-mouse CD40L ( $1 \mu \mathrm{g} / \mathrm{ml})$, anti-mouse IgM $(5 \mu \mathrm{g} / \mathrm{ml})$, LPS 
$(10 \mu \mathrm{g} / \mathrm{ml})$ and OVA-biotin tetramers $(0,2 \mu \mathrm{g} / \mathrm{ml}+0.5 \mu \mathrm{g} / \mu \mathrm{l}$ Streptavidin-PE). $200 \mu \mathrm{l}$ of the cell suspension were applied to a round bottom 96-well plate and incubated for $72 \mathrm{~h}$ at standard conditions. Differentiation into plasma cells was assessed by FACS analyses of surface immunoglobulin and plasma cell markers, as well as Enzyme Linked Immunosorbent Assay (ELISA) of specific immunoglobulins.

\subsubsection{Phenotypical and Functional Analyses}

\subsubsection{Fluorescence Activated Cell Sorting}

Acquisition and enumeration of cells were performed by using a Gallios Flow Cytometer (Beckman Coulter, Pasadena, USA). Single-cell analyses were performed by use of the FlowJo software (Tree Star, Ashland, USA) or the Kaluza software (Beckman Coulter, Pasadena, USA). All washing steps were performed with cell wash in a Heraeus Megafuge 16R (Thermo Scientific) at $270 \times \mathrm{g}$ for $5 \mathrm{~min}$ at RT.

\subsection{Surface Staining with Monoclonal Antibodies}

Surface staining with monoclonal antibodies for FACS analyses was performed by washing $0.1-1 \times 10^{6}$ cells in $4 \mathrm{ml}$ cell wash. Cells were resuspended in $100 \mu \mathrm{l}$ cell wash and stained with $1 \mu \mathrm{l}$ of antibody solution for $20 \mathrm{~min}$ at $4{ }^{\circ} \mathrm{C}$. Afterwards, cells were washed and resuspended in 150 - $300 \mu \mathrm{l}$ cell wash depending on the cell number. Monoclonal antibodies used for staining, conjugates and companies are listed in table 2.1.

Table 2.1. Monoclonal antibodies used for FACS analyses.

\begin{tabular}{|c|c|c|}
\hline Antibody & Conjugate & Company \\
\hline \multicolumn{3}{|c|}{ Murine Antibodies } \\
\hline \multirow[t]{2}{*}{ B220 } & Alexa Fluor 750 & Life Technologies \\
\hline & Pacific Blue & Biolegend \\
\hline CD3 & APC-Cy7 & $B D$ \\
\hline CD4 & PE-Cy7 & $\mathrm{BD}$ \\
\hline $\mathrm{CD} 8$ & PerCP-Cy5.5 & $\mathrm{BD}$ \\
\hline CD11b & APC-Су7 & $B D$ \\
\hline CD11c & $\mathrm{PE}$ & $B D$ \\
\hline \multirow[t]{2}{*}{ CD19 } & Alexa Fluor 700 & Biolegend \\
\hline & PE-Texas Red & Life Technologies \\
\hline CD27 & PE-Cy7 & Biolegend \\
\hline CD80 & APC & BD \\
\hline CD86 & FITC & $B D$ \\
\hline
\end{tabular}


Material and Methods

\begin{tabular}{|c|c|c|}
\hline & Alexa Fluor 700 & Biolegend \\
\hline CD138 & PerCP-Cy5.5 & Biolegend \\
\hline \multirow[t]{2}{*}{$\operatorname{lgG1}$} & FITC & $\mathrm{BD}$ \\
\hline & PE-Vio770 & Miltenyi Biotech \\
\hline $\operatorname{lgM}$ & APC & Biolegend \\
\hline \multirow[t]{2}{*}{$\lg D$} & PerCP-Cy5.5 & Biolegend \\
\hline & VioBlue & Miltenyi Biotech \\
\hline $\mathrm{MHCl}$ & FITC & eBioscience \\
\hline $\mathrm{IA}^{\mathrm{b}}(\mathrm{MHC} \mathrm{II})$ & FITC & Biolegend \\
\hline Vß 5.1, 5.2 & $\mathrm{PE}$ & $\mathrm{BD}$ \\
\hline \multicolumn{3}{|c|}{ Human Antibodies } \\
\hline CD3 & APC-Cy7 & Beckman Coulter \\
\hline CD4 & ECD & Beckman Coulter \\
\hline CD8 & FITC & $\mathrm{BD}$ \\
\hline CD11b & Alexa Fluor 700 & Biolegend \\
\hline CD11c & APC & Biolegend \\
\hline CD19 & APC eFluor 780 & eBioscience \\
\hline CD20 & Pacific Orange & Life Technologies \\
\hline CD21 & APC & Biolegend \\
\hline CD27 & PE-Cy7 & Biolegend \\
\hline CD80 & FITC & BD \\
\hline CD86 & Pacific Blue & Biolegend \\
\hline CD138 & Alexa Fluor 700 & Biolegend \\
\hline $\operatorname{lgD}$ & FITC & $\mathrm{BD}$ \\
\hline $\operatorname{IgG1}$ & ECD & Beckman Coulter \\
\hline IgM & PerCP-Cy5.5 & Biolegend \\
\hline HLA-DR & Alexa Fluor 700 & eBioscience \\
\hline \multicolumn{3}{|c|}{ Species Independent Antibodies } \\
\hline Annexin & $\mathrm{PE}$ & $\mathrm{BD}$ \\
\hline \multirow[t]{2}{*}{ Streptavidin } & $\mathrm{PE}$ & $\mathrm{BD}$ \\
\hline & APC & Biolegend \\
\hline
\end{tabular}

\subsection{Staining of Antigen-Specific B Cells with Antigen Tetramers}

Antigen-specific B cells were identified by staining with antigen tetramers. In order to form tetramers, $0.2 \mu \mathrm{g}$ of biotinylated OVA- or HBV-protein was pre-incubated with $0.5 \mu \mathrm{g}$ of Streptavidin-PE for 5 min at RT. Afterwards, antigen-tetramers were incubated together with surface antibodies (chapter 2.2.3.1.1). 


\subsubsection{Mixed-Lymphocyte Reaction}

To study the APC capacity of CD40B cells and DCs, antigen-specific autologous mixedlymphocyte reactions (MLR) were performed.

APCs were incubated with the specific antigen at a concentration of $75 \mathrm{nM}$ for $24 \mathrm{~h}$ prior to incubation with the T cells. Afterwards, APCs were harvested and resuspended in HeLa standard medium at a concentration of $1 \times 10^{6}$ cells $/ \mathrm{ml}$. Cells were irradiated once with 26 Gy to stop them from proliferation. Serial dilutions were performed for incubation with $T$ cells at APC-to-T cell ratios 3:1, 1:1, 1:5, 1:10, 1:20 and 1:50 in $100 \mathrm{ml}$ HeLa standard medium.

$\mathrm{CD}^{+} \mathrm{T}$ cells were isolated from spleens of OT-I or OT-II mice by positive selection (chapter 2.2.1.4). For detection of $T$ cell proliferation, the $T$ cells were stained with the fluorescent proliferation marker CFSE. For this purpose, $\mathrm{CD}^{+} \mathrm{T}$ cells were resuspended at a concentration of $5 \times 10^{6}$ cells/ $\mathrm{ml}$ in $1 \times$ PBS with $5 \%$ FBS, stained with $10 \mu \mathrm{M}$ CFSE and incubated for $5 \mathrm{~min}$ in the dark with constant agitation. The staining reaction was stopped by adding $5 \mathrm{ml}$ of pure FBS and cells were centrifuged at $270 \times \mathrm{g}$ for $5 \mathrm{~min}$. T cells were resuspended at a concentration of $1 \times 10^{6} \mathrm{cells} / \mathrm{ml}$ in HeLa standard medium. T cells were plated in $100 \mu \mathrm{l}$ at indicated APC-to-T cell ratios on a round bottom 96-well plate. Cocultures were incubated for 5 days at standard culture conditions. CFSE segregates equally between daughter cells upon cell division, resulting in sequential reduction of cellular fluorescence intensity with each successive generation. Therefore, proliferation of $\mathrm{T}$ cells was detected by determining the percentage of proliferating $\mathrm{CFSE}^{\text {low }} \mathrm{CD}^{+} \mathrm{CD}^{+}$or $\mathrm{CD} 8^{+} \mathrm{T}$ cells by FACS analysis (chapter 2.2.3.1). Activation of $\mathrm{T}$ cells was determined by positive staining for the $T$ cell activation marker CD25.

\subsubsection{Enzyme-Linked Immunosorbent Assay}

Secretion of specific antibodies in plasma cell differentiation assays was determined in ELISAs for OVA-specific IgG1 antibodies. For this purpose, 96-well high-binding plates were incubated with OVA-protein at a concentration of $40 \mu \mathrm{g} / \mathrm{ml}$ in a volume of $50 \mu \mathrm{l}$ Coating Buffer. The plate was sealed and incubated over night at $4{ }^{\circ} \mathrm{C}$. The day after, the protein solution was discarded into a sink and the plate was washed three times with PBS/Tween. Afterwards, non-specific binding was blocked by incubating the plate with $100 \mu$ Blocking 
Solution for $1 \mathrm{~h}$ at RT. The plate was washed three times with PBS/Tween before applying 50 $\mu$ of standards and samples in doublets. Samples were diluted 1:5 in Blocking Solution. Standards were applied at $5 \mathrm{ng} / \mathrm{ml}, 10 \mathrm{ng} / \mathrm{ml}, 50 \mathrm{ng} / \mathrm{ml}, 100 \mathrm{ng} / \mathrm{ml}, 250 \mathrm{ng} / \mathrm{ml}, 500 \mathrm{ng} / \mathrm{ml}$, $1000 \mathrm{ng} / \mathrm{ml}, 2500 \mathrm{ng} / \mathrm{ml}$ and $5000 \mathrm{ng} / \mathrm{ml}$ in Blocking Solution. The plate was sealed and incubated at RT for 2-4 h. Afterwards the plate was washed three times with PBS/Tween and $50 \mu \mathrm{l}$ of the detection antibody anti-mouse IgG1 was added at a concentration $100 \mathrm{ng} / \mathrm{ml}$ in Blocking Solution. The plate was sealed and incubated at RT for $1 \mathrm{~h}$. The plate was washed three times before adding the avidin-horseradish peroxidase (Av-HRP) at a dilution of 1:1000 in Blocking Solution. The plate was incubated for $30 \mathrm{~min}$ at RT. Afterwards, the plate was washed 5 times with PBS/Tween before applying $50 \mu \mathrm{l}$ of the premixed ABTS substrate solution A and B. The enzymatic reaction was stopped with $50 \mu \mathrm{l} 1 \%$ SDS and the extinction at $405 \mathrm{~nm}$ was measured in a $\mu$-Quant microplate reader.

\subsubsection{In Vivo Experiments}

The animal experiments were performed in accordance with the national and European guidelines for laboratory animal keeping with permission from the local government authorities (permission number 84-02.04.2011.A226). Mice were bred in the animal facility of the Department of Pathology at the University Hospital Cologne under specific pathogenfree conditions.

C57BL/6NRj wild type mice were obtained from Janvier Labs (France) and then further bred in our own animal facility.

C57BL/6-Tg(TcraTcrb)1100Mjb/Crl (OT-I) mice contain inserts for mouse Tcra-V2 and Tcrb-V5 genes and express transgenic T cell receptors (TCR) that recognize ovalbumin residues 257264 in the context of H2Kb. OT-I mice were routinely monitored for expression of TCR-chain $\mathrm{V} \beta 5$ on $\mathrm{CD}^{+} \mathrm{CD}^{+} \mathrm{T}$ cells by FACS analysis.

C57BL/6-Tg(TcraTcrb)425Cbn/Crl (OT-II) mice express the mouse alpha-chain and beta-chain T cell receptor that pairs with the CD4 coreceptor and is specific for chicken ovalbumin 323339 in the context of $\mathrm{I}-\mathrm{A}^{\mathrm{b}}$. They show a four-fold increase in the $\mathrm{CD} 4^{+}$to $\mathrm{CD} 8^{+}$peripheral $\mathrm{T}$ cell ratio. OT-II mice were routinely monitored for expression of TCR-chain $\mathrm{V} \beta 5$ on $\mathrm{CD} 3^{+} \mathrm{CD} 4^{+}$ T cells by FACS analysis. 
Luciferase $^{+}\left(\right.$LuC $\left.^{+}\right)$mice were on a C57BL/6N background and were kindly provided by Prof.

Dr. Robert Zeiser (Laboratory for Allo-Immunregulation, Department for Internal Medicine I, University Hospital Freiburg). These mice express the firefly luciferase under control of the $\beta$-actin promoter in many leukocyte subsets including $C D 19^{+} B 220^{+} B$ cells.

\subsubsection{Vaccination Strategies}

\subsection{Immunization with Peptide-Loaded Cell Subsets}

In order to immunize $C D 57 \mathrm{BL} / 6 \mathrm{~N}$ mice with $\mathrm{CD} 40 \mathrm{~B}$ cells or mature $\mathrm{DCs}$, cell subsets were exogenously loaded with OVA protein. For this purpose, CD40B cells or DCs were harvested and washed with serum-free medium at $270 \times \mathrm{g}$ for $5 \mathrm{~min}$. Cells were suspended in serumfree medium at a concentration of $1 \times 10^{6}$ cells $/ \mathrm{ml}$ and incubated with $10 \mu \mathrm{M}$ OVA-Protein for $1 \mathrm{~h}$ at standard culture conditions. Before injection, cells were washed three times with $20 \mathrm{ml} 1 \times$ PBS. Cells were injected in $100 \mu \mathrm{l} 1$ x PBS. Cell numbers and injection routes were as indicated in experiments.

\subsection{Immunization with Incomplete Freund's Adjuvant}

C57BL/6N or Luc ${ }^{+}$mice were immunized with $20 \mu \mathrm{M}$ OVA-Protein in Incomplete Freund's Adjuvant (IFA) for generation of OVA-specific B cells. In order to prepare the IFA/protein emulsion, OVA-Protein was dissolved in PBS and added to the IFA in a ratio of 1:1. The solution was strongly vortexed until it appeared white and viscous. The emulsion was transferred to the syringes without attaching the needle. The needle was added immediately prior to injection. $100 \mu$ of the protein/IFA solution was injected i.p.

As positive control for in vivo cytotoxicity assays and tumor control, mice were immunized with OVA-Protein/ IFA solutions in addition of $7 \mu \mathrm{M}$ immunomodulatory CpG-ODN 2395 (oligodeoxynucleotide with non-methylated cytosine-guanine motifs). Positive controls were injected i.p.

\subsubsection{In Vivo Cytotoxicity Assays}

In vivo cytotoxicity (cytotox) assays aim to detect in vivo killing of antigen-presenting target cells by recognition through cognate $\mathrm{CD}^{+} \mathrm{T}$ cells induced by immunization (chapter 
2.2.4.1.1). Five to seven days after the last immunization, mice were injected with CFSElabeled target cells from naïve syngeneic mice loaded with OVA-peptide (aa 257 - 264).

\subsubsection{Injection of Target Cells}

Target cells were prepared from single cell suspension from spleens of 7-18 week old C57BL/6 mice (chapter 2.2.1.1). Splenocytes were washed two times with 1x PBS at $270 \times \mathrm{g}$ for 5 min. Afterwards, cells were resuspended in 1x PBS with $5 \%$ FBS at a concentration of $100 \times 10^{6}$ cells $/ \mathrm{ml}$. Splenocytes were split into fractions. One fraction was labeled with a low amount of CFSE $(2 \mu \mathrm{M})$ and one fraction was labeled with high amount of CFSE $(20 \mu \mathrm{M})$ by constantly agitating the tubes for $5 \mathrm{~min}$ in the dark. The labeling reaction was stopped by adding $5 \mathrm{ml}$ pure FBS. The cells were washed at $270 \times \mathrm{g}$ for $5 \mathrm{~min}$ and resuspended in serumfree medium at a concentration of $1 \times 10^{7}$ cells $/ \mathrm{ml}$. The CFSE high fraction was pulsed with $10 \mu \mathrm{M}$ OVA-peptide, the CFSE low fraction was left unpulsed. Cells were incubated for $1 \mathrm{~h}$ at standard conditions. Afterwards, both fractions were washed extensively by resuspending them in $20 \mathrm{ml} \mathrm{PBS}$ and centrifuging at $270 \times \mathrm{g}$ for $5 \mathrm{~min}$. For injection, cells were resuspended at a concentration of $10 \times 10^{7}$ cells $/ \mathrm{ml}$ in $1 \times$ PBS and both fractions were

\subsubsection{Detection of Specific Cytolysis}

To analyze remaining CFSE-positive target cell fractions in immunized mice, spleens were isolated 24 hours after target cell injection. Spleen were minced through a $100 \mu \mathrm{m}$ cell strainer and resuspended in $1 \times$ PBS. Splenocytes were counted and $4 \times 10^{6}$ cells were filled in a FACS tube, washed and resuspended in $300 \mu \mathrm{l}$ cell wash. Cells were analyzed by FACS immediately. The ratio of unpulsed versus pulsed (RatioUP) target cells was determined by dividing the percentage of CFSE low cells by the percentage of CFSE high cells. The percentage of the specific lysis was then calculated by the following formula:

\% Specific Lysis = (1-(RatioUP Negative Control/RatioUP Immunized $)) * 100$

\subsubsection{In Vivo Homing Studies}

For in vivo migrations studies of polyclonal B cells, B cells were isolated from spleens of Luc $^{+}$ mice (chapter 2.2.1.1) and activated in the CD40 culture system (chapter 2.2.2.7). For migration studies of OVA-specific B cells, $\mathrm{Luc}^{+}$mice were immunized with OVA-Protein 14 
days before isolation (chapter 2.2.4.1.2). Luc $^{+}$mice were injected i.v. into 7-12 weeks old C57BL/6N wild type mice. Detection of $\mathrm{Luc}^{+} \mathrm{B}$ cells was performed by injecting $7.5 \mathrm{mg} \mathrm{D}$ luciferin in $250 \mathrm{ml}$ 1x PBS i.p. into wild type mice. The luciferin was allowed to distribute in the mouse for 5 min before mice were narcotized with 1.5-4 \% isofluran. Mice were shaved prior to imaging in order to minimize interference by the fur. Imaging was performed in the Xenogen IVIS 200 (Perkin Elmer). Mice were constantly kept under narcosis with 1.5-4 \% isofluran at $37^{\circ} \mathrm{C}$. Bioluminescence pictures were analyzed with the Living Image Software (Perkin Elmer).

\subsubsection{Tumor Formation}

For tumor control experiments, E.G7 lymphoma cells (chapter 2.2.2.6.3) were kept at a low concentration of $1 \times 10^{6}$ cells $/ \mathrm{ml}$ prior to injection. For tumor formation, cells were harvested and resuspended at a concentration of $4 \times 10^{6}$ cells $/ \mathrm{ml}$ in $1 \times \mathrm{PBS} .100 \mu \mathrm{l}\left(0.4 \times 10^{6}\right.$ cells) were injected s.c. into the right flank of immunized or naïve $\mathrm{C} 57 \mathrm{BL} / 6 \mathrm{~N}$ mice. Tumor size was determined daily from day 7 after inoculation by measuring tumor diameter in two dimensions using a vernier caliper. The tumor volume was calculated using the following formula:

Tumor volume $=0.5 \times\left(\right.$ length $\times$ width $\left.^{2}\right)$

Tumors were allowed to grow for 40 days or until one diameter reached a size of $15 \mathrm{~mm}$.

\subsubsection{Statistics}

Significant differences were calculated by ordinary one-way ANOVA or ordinary two-way ANOVA were appropriate using GraphPad Prism Software. P-values of less than 0.05 were considered statistically significant and marked with asterisks: $* p \leq 0.05, * * p \leq 0.01, \quad * * * p$ $\leq 0.001, * * * * p \leq 0.0001$. Mean values and standard deviations (SD) were calculated from at least 3 independent experiments. 

Results 



\section{Results}

\subsection{Antigen-Specific B Cells can be Isolated by Antigen Tetramers}

Study of antigen-specific B cells is challenging, because of low frequencies in peripheral blood or spleens and low signaling by fluorescently labeled antigens (Franz et al. 2011). However, a sensitive method enabling the detection of B cells with defined specificity in FACS analyses are antigen tetramers. They are produced by biotinylation of the soluble antigen and tetramerization with fluorescently labeled streptavidin (Fig. 3.1).

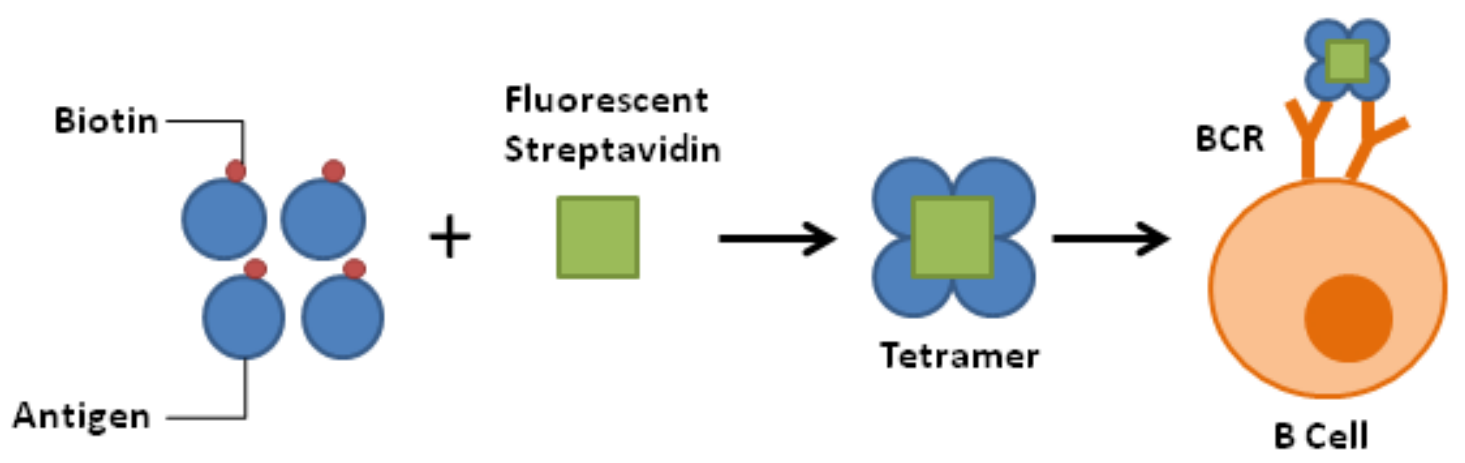

Fig. 3.1 Overview of the antigen tetramer staining. Soluble antigen is biotinylated and incubated with fluorescently labeled streptavidin to form tetramers. B cells are stained with antigen tetramers and a panel of surface antibodies.

\subsubsection{Murine Antigen-Specific B Cells can be Purified by Antigen-Tetramers}

Under normal conditions, the frequency of B cells with defined specificity in spleen is below $1 \%$ (Oshiba et al. 1994, Kodituwakku et al. 2003). In order to increase the number of OVAspecific B cells, C57BL/6 mice were immunized with $20 \mu \mathrm{M}$ OVA-protein in IFA. This increased the percentage of OVA-specific $B$ cells among all CD19 ${ }^{+} B 220^{+} B$ cells to $2-9 \%$ in spleens (Fig. 3.2A, middle plot) and to about $30 \%$ in inguinal lymph nodes (Fig. 3.2B, right plot), which was determined by labeling with antigen tetramers and FACS analyses (Fig. 3.1). However, the percentage of OVA-specific B cells in the inguinal lymph nodes (LN) varied greatly from 20 to $90 \%$ (data not shown). 
A

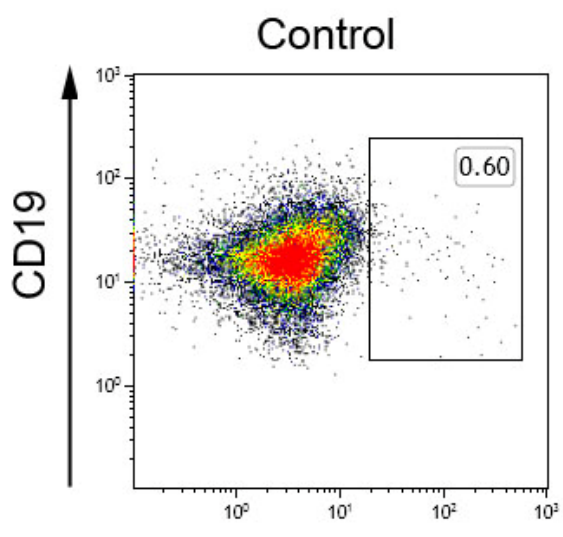

Immunized before purification

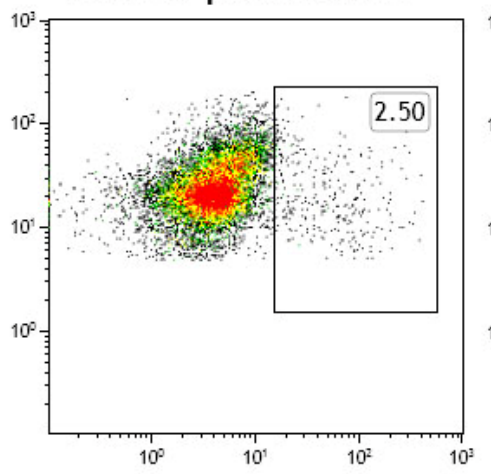

Immunized after purification

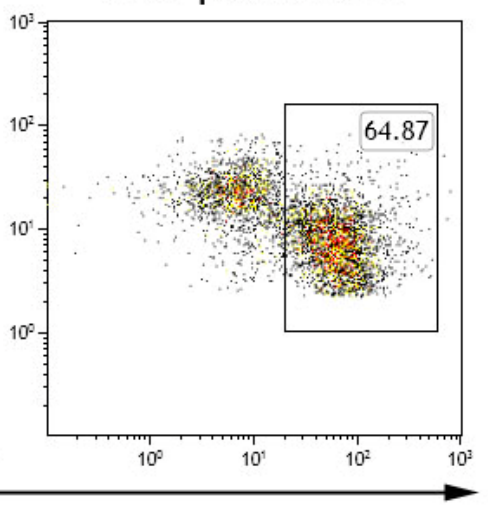

OVA

B

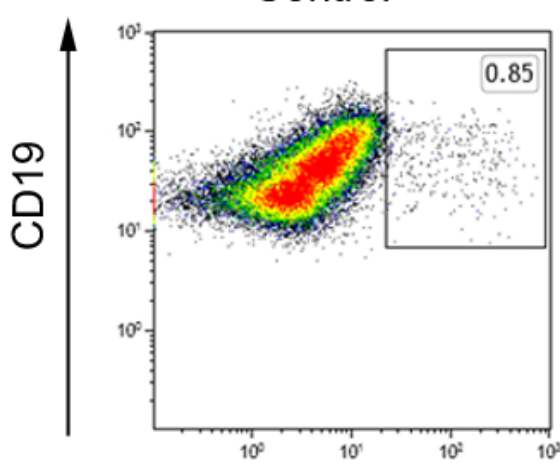
Non-Draining LN
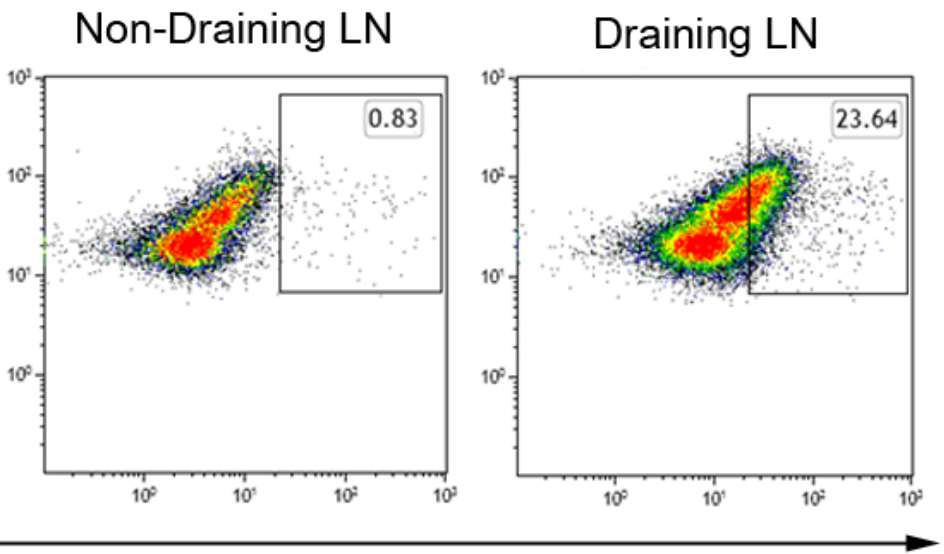

OVA

Fig. 3.2 Percentage of OVA-specific B cells. (A) Splenocytes from mice were stained for OVA-specific $B$ cells among the $\mathrm{CD} 19^{+} \mathrm{B} 220^{+} \mathrm{B}$ cell population with OVA-Biotin tetramers. Representative flow cytometry analyses out of at least 10 independent experiments with non-immunized mice (control, left plot) or immunized mice (middle plot) are shown. Splenocytes from immunized mice were purified by positive selection with OVA-Biotin tetramers (right plot). (B) Single cell suspensions from inguinal and axillary lymph were stained for OVA-specific B cells among the $C D 19^{+} B 220^{+}$B cell population with OVA-Biotin tetramers. Representative flow cytometry analyses out of at least 10 independent experiments of inguinal lymph nodes (draining LN, right plot) and axillary lymph nodes (non-draining LN, middle plot) with immunized mice are shown. Inguinal lymph nodes of nonimmunized mice served as control (left plot). Numbers indicate the percentage of OVA ${ }^{+} B$ cells.

OVA-specific B cells could be enriched from splenocytes by positive selection via antigen tetramers. The efficiency of the enrichment strongly depended on the grade of biotinylation and therefore varied between 20 and $70 \%$. However, under optimal conditions an OVAspecific B cell purity of above $60 \%$ of all $\mathrm{CD}_{19^{+}} \mathrm{B}_{220^{+}} \mathrm{B}$ cells could be reached (Fig. 3.2A, right plot). The overall purity of $\mathrm{CD} 19^{+} \mathrm{B} 220^{+} \mathrm{B}$ cells was around $80 \%$ (Fig. 3.3), but was never higher than $85 \%$ unlike with $\mathrm{CD} 19^{+}$selection of $\mathrm{B}$ cells. Higher $\mathrm{B}$ cell purity could also 
not be reached by negative $B$ cell selection prior to OVA-specific enrichment (data not shown).

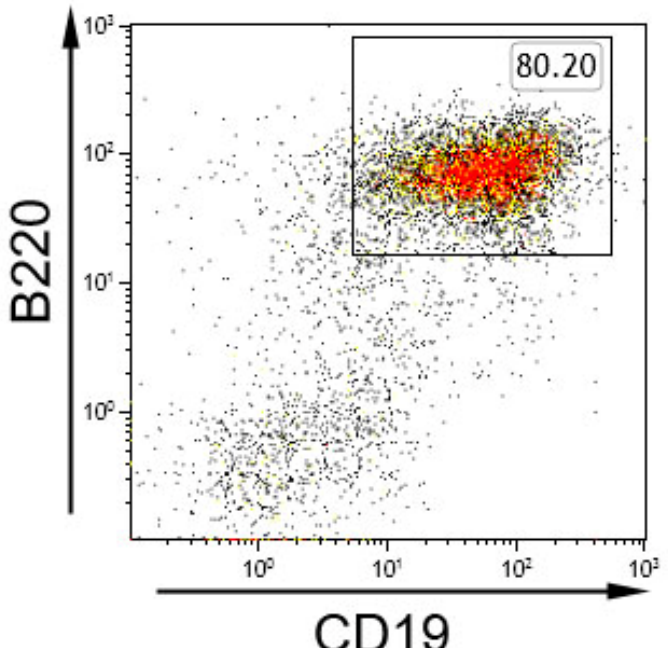

Fig. 3.3 B cell purity of OVA-enriched B cells. Representative flow cytometry analysis of purified OVA-specific B cells out of at least 10 independent experiments is shown. Splenocytes from immunized mice were purified by positive selection with OVA-Biotin tetramers and stained for $\mathrm{CD} 19^{+} \mathrm{B} 220^{+} \mathrm{B}$ cells. Numbers indicate the percentage of $\mathrm{CD} 19^{+} \mathrm{B} 220^{+} \mathrm{B}$ cells.

B cells of other specificities could also be generated and isolated, which was demonstrated by isolation of Keyhole Limpet Hemocyanin (KLH)-specific B cells. According to the generation of OVA-specific B cells, KLH-specific B cells were isolated from immunized mice and stained with $\mathrm{KLH}$ tetramers. About 2-9\% of all $\mathrm{CD} 19^{+} \mathrm{B} 220^{+} \mathrm{B}$ cells in spleens were specific for KLH (Fig. 3.4, middle plot). KLH-specific B cells were enriched using a Memory B cell Isolation Kit, which resulted in a purity of KLH-specific B cells of about $7 \%$ (Fig. 3.4, right plot).

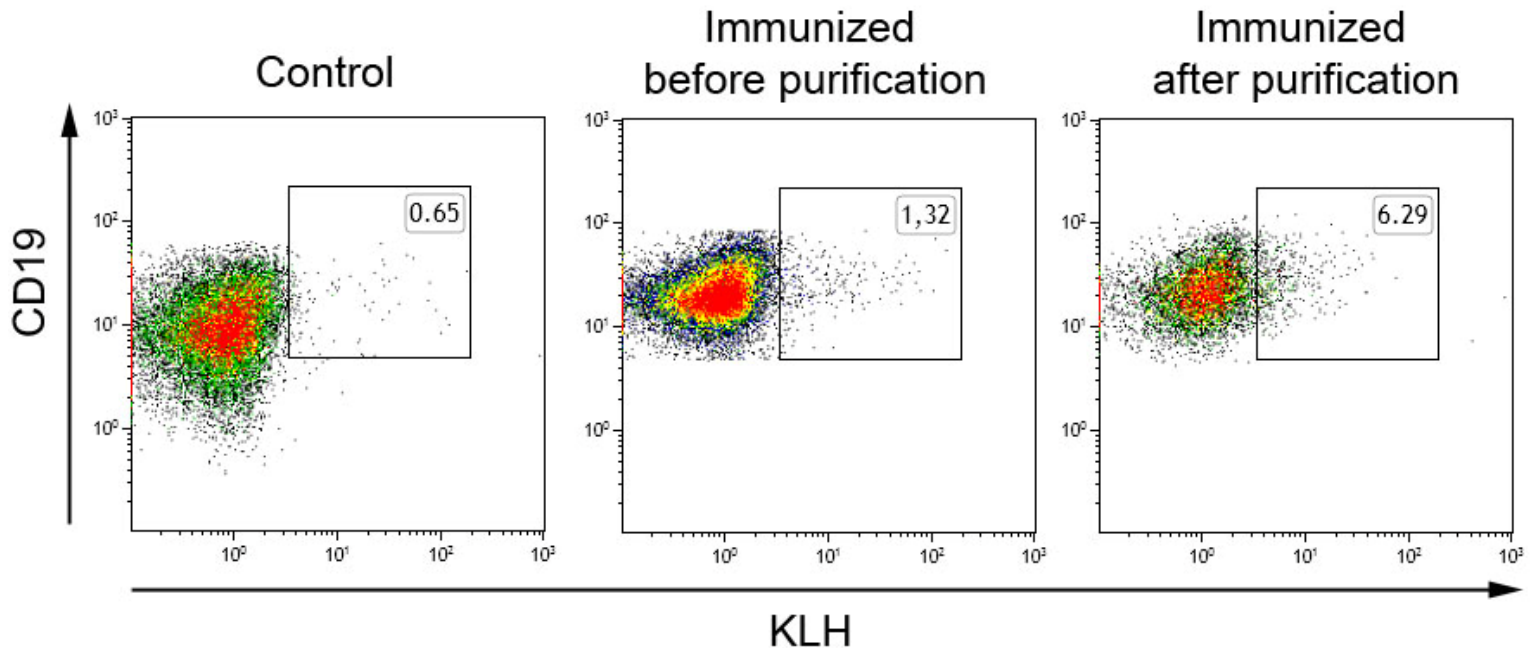

Fig. 3.4 Percentage of KLH-specific B cells. Splenocytes from mice were stained for KLH-specific B cells among the $\mathrm{CD}_{19}{ }^{+} \mathrm{B} 22 \mathrm{O}^{+} \mathrm{B}$ cell population with KLH-Biotin tetramers. Representative flow cytometry analyses out of 3 independent experiments with non-immunized mice (control, left plot) or immunized mice (middle plot) are shown. Splenocytes from immunized mice were purified by memory B cell selection (right plot). Numbers indicate the percentage of $\mathrm{KLH}^{+} \mathrm{B}$ cells. 


\subsubsection{Murine Antigen-Specific B Cells Show a Class-Switched Phenotype}

The isolated antigen-specific B cells were characterized by FACS analyses for several surface markers defining their activation status and developmental state. Bar charts represent results of at least three independent experiments. Expression levels of B cells of immunized mice were normalized to the expression levels of B cells of non-immunized (control) mice.

There was no difference in expression of the B cell marker B220 in OVA-specific CD19+ $B 220^{+}$ B cells compared to control B cells of non-immunized mice or OVA-negative B cells of immunized mice (Fig. 3.5).

A

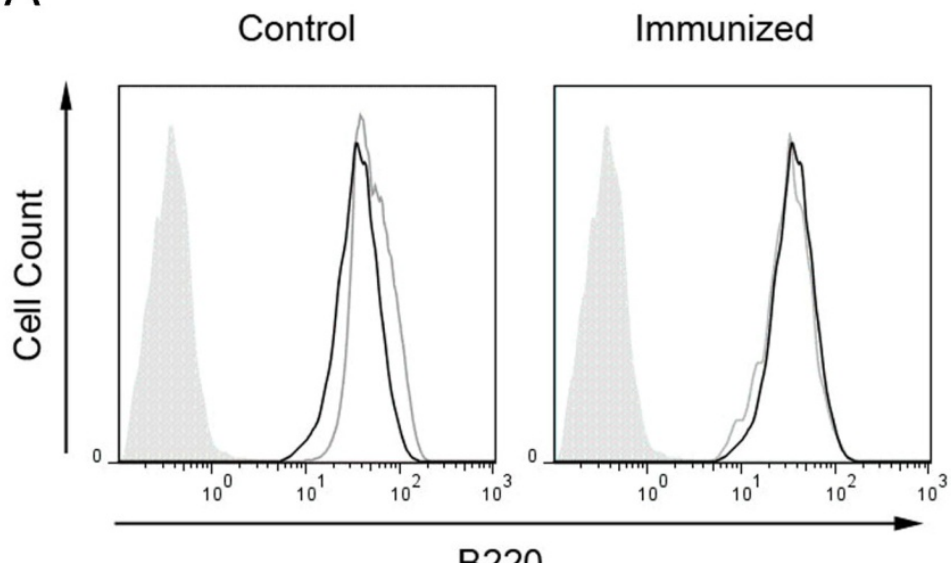

$\mathrm{B}$

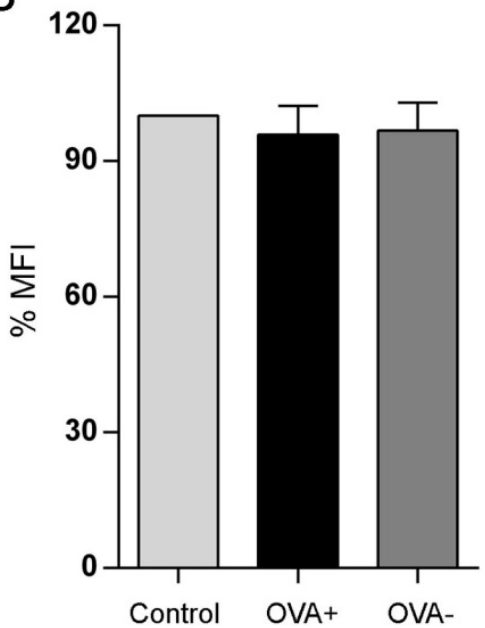

Fig. 3.5 B220 expression in OVA-specific B cells. OVA-specific B cells from immunized mice were stained for their expression of B220 and analyzed by flow cytometry. (A) Representative analyses out of 5 independent experiments are shown. OVA-specific B cells (black line) were compared to $B$ cells of non-immunized (control) mice (left plot, grey line) and OVA-negative B cells of immunized mice (right plot, grey line). Cells that were analyzed without antibody staining served as unstained control (filled histogram). (B) The mean fluorescent intensity (MFI) of B220 of OVA-specific (OVA+) and OVAnegative (OVA-) B cells in immunized mice were normalized to the expression levels in $B$ cells of control mice. Bar charts show mean values \pm SEM of three independent experiments. Significant differences calculated with ordinary one-way ANOVA were not detected.

Although the B cell marker CD19 was upregulated in immunized mice, the difference was not significant (Fig. 3.6). Phenotyping of KLH-specific B cells revealed similar results (data not shown). 
A

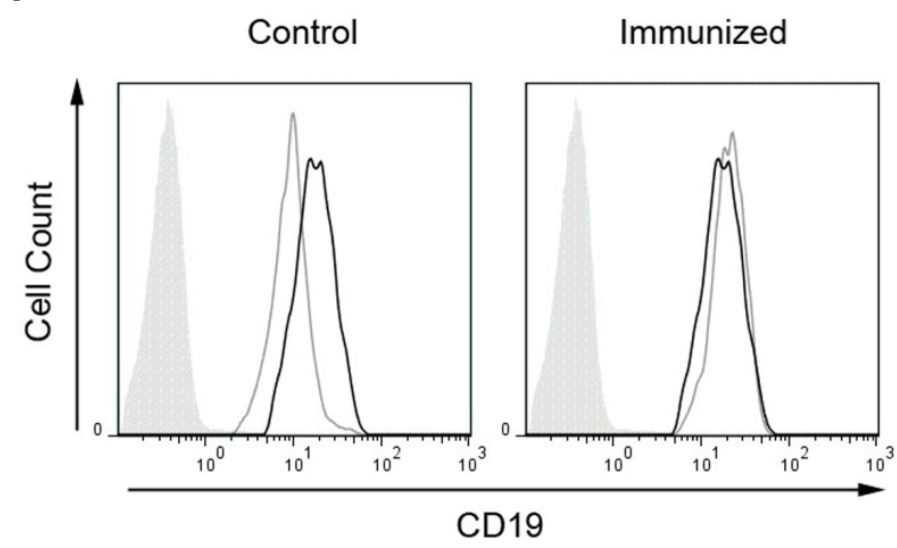

B

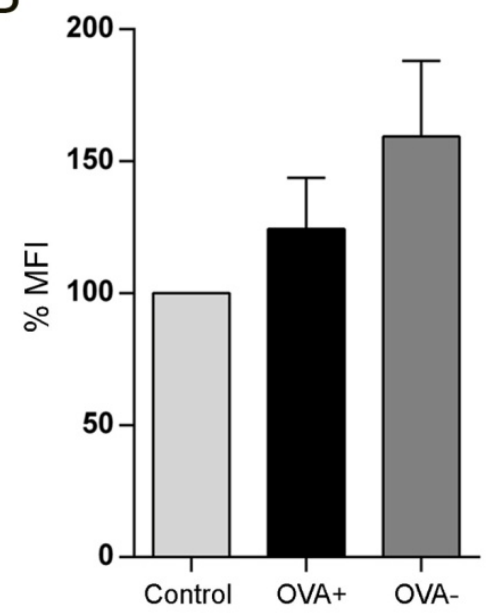

Fig. 3.6 CD19 expression in OVA-specific B cells. OVA-specific B cells from immunized mice were stained for their expression of CD19 and analyzed by flow cytometry. (A) Representative analyses out of 5 independent experiments are shown. OVA-specific B cells (black line) were compared to $B$ cells of non-immunized (control) mice (left plot, grey line) and OVA-negative $B$ cells of immunized mice (right plot, grey line). Cells that were analyzed without antibody staining served as unstained control (filled histogram). (B) The mean fluorescent intensity (MFI) of CD19 of OVA-specific (OVA+) and OVAnegative (OVA-) $B$ cells in immunized mice were normalized to the expression levels in $B$ cells of control mice. Bar charts show mean values \pm SEM of three independent experiments. Significant differences calculated with ordinary one-way ANOVA were not detected.

Interestingly, the percentage of $\operatorname{lgD}^{+}$and $\operatorname{IgM}^{+}$OVA-specific $\mathrm{CD} 19^{+} \mathrm{B} 22 \mathrm{O}^{+} \mathrm{B}$ cells was significantly decreased ( $22.41 \% \pm 18.81$ and $26.37 \% \pm 19.91$, respectively) compared to control B cells of non-immunized mice $(90.33 \% \pm 1.74$ and $82.09 \% \pm 14.98$, respectively) and OVA-negative B cells of immunized mice $(60.20 \% \pm 20.93$ and $77.16 \% \pm 13.44$, respectively; Fig. 3.7 and Fig. 3.8). In contrast, IgG1 ${ }^{+}$OVA-specific B cells were significantly increased $(77.25 \% \pm 18.17)$ compared to control B cells of non-immunized mice $(6.98 \% \pm$ 7.98) or OVA-negative B cells of immunized mice (51.95 \% \pm 2.90 ; Fig. 3.9). This clearly demonstrated a class-switched phenotype of the isolated B cells, confirming the assumption that they are antigen-specific B cells. KLH-specific B cells showed similar percentaged distribution of immunoglobulins (data not shown). 
A

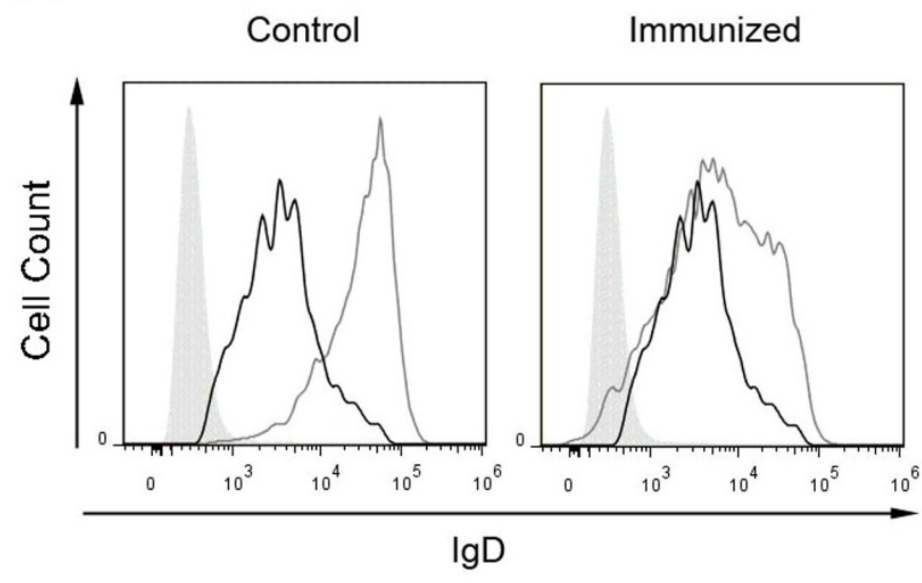

B

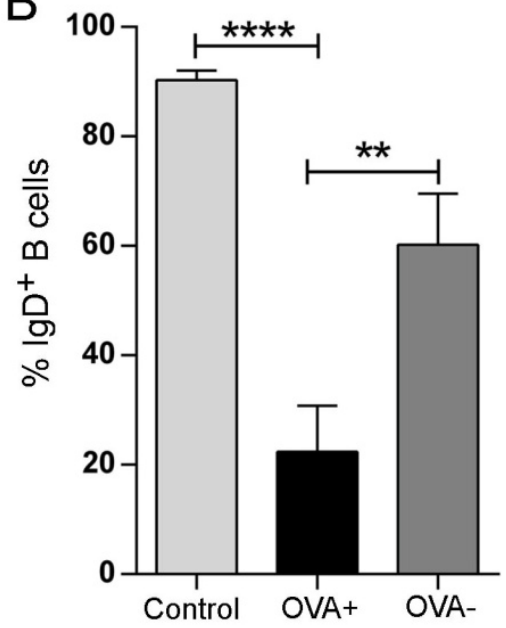

Fig. 3.7 Percentage of IgD ${ }^{+}$B cells. OVA-specific B cells from immunized mice were stained for their expression of IgD and analyzed by flow cytometry. (A) Representative analyses out of 5 independent experiments are shown. OVA-specific B cells (black line) were compared to B cells of non-immunized (control) mice (left plot, grey line) and OVA-negative B cells of immunized mice (right plot, grey line). Cells that were analyzed without antibody staining served as unstained control (filled histogram). (B) The percentage of IgD ${ }^{+} B$ cells among OVA-specific (OVA+), OVA-negative (OVA-) or B cells of control mice are shown. Bar charts show mean values \pm SEM of three independent experiments. Significant differences were calculated with ordinary one-way ANOVA are marked by an asterisk. ${ }^{* *} p \leq 0.01$, $* * * * p \leq 0.0001$.

A

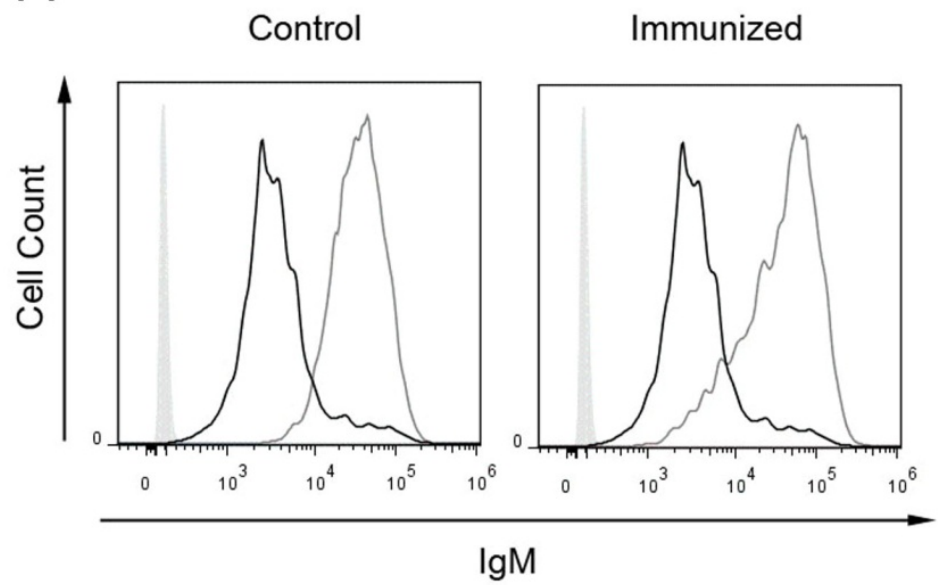

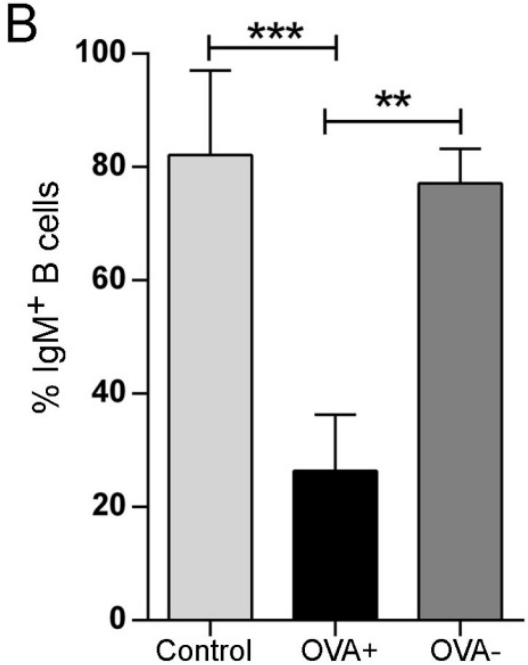

Fig. 3.8 Percentage of IgM $^{+}$B cells. OVA-specific B cells from immunized mice were stained for their expression of IgM and analyzed by flow cytometry. (A) Representative analyses out of 5 independent experiments are shown. OVA-specific B cells (black line) were compared to B cells of non-immunized (control) mice (left plot, grey line) and OVA-negative B cells of immunized mice (right plot, grey line). Cells that were analyzed without antibody staining served as unstained control (filled histogram). (B) The percentage of IgM $^{+} B$ cells among OVA-specific (OVA+), OVA-negative (OVA-) or B cells of control mice are shown. Bar charts show mean values \pm SEM of three independent experiments. Significant differences were calculated with ordinary one-way ANOVA are marked by an asterisk. ${ }^{* *} p \leq 0.01$, $* * * p \leq 0.001$. 
A

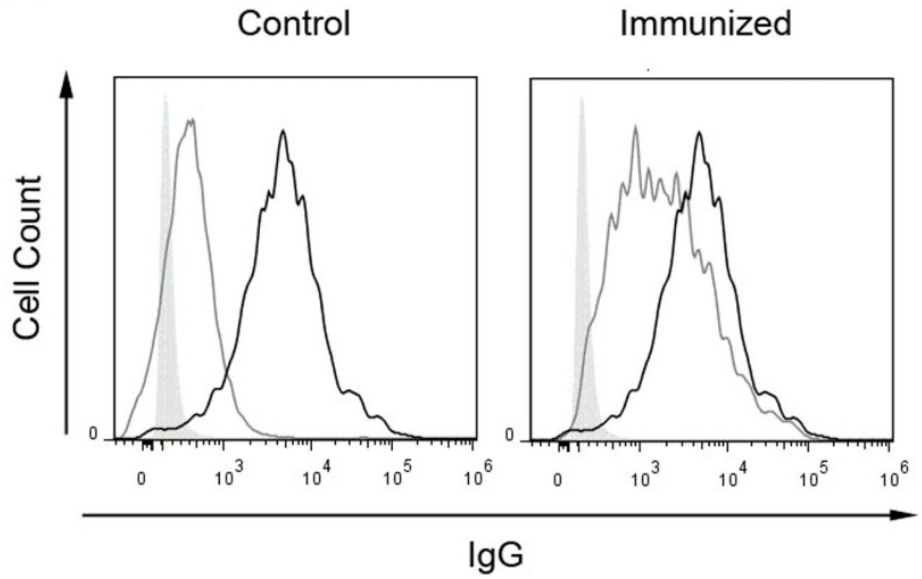

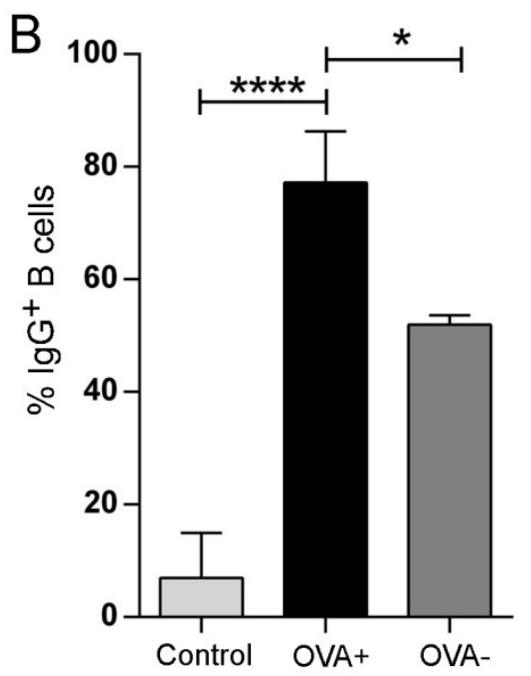

Fig. 3.9 Percentage of IgG ${ }^{+}$B cells. OVA-specific B cells from immunized mice were stained for their expression of IgG and analyzed by flow cytometry. (A) Representative analyses out of 5 independent experiments are shown. OVA-specific B cells (black line) were compared to B cells of non-immunized (control) mice (left plot, grey line) and OVA-negative B cells of immunized mice (right plot, grey line). Cells that were analyzed without antibody staining served as unstained control (filled histogram). (B) The percentage of IgG ${ }^{+} B$ cells among OVA-specific (OVA+), OVA-negative (OVA-) or B cells of control mice are shown. Bar charts show mean values \pm SEM of three independent experiments. Significant differences were calculated with ordinary one-way ANOVA are marked by an asterisk. ${ }^{*} p \leq 0.05$, $* * * * p \leq 0.0001$.

OVA-specific B cells show significantly higher expression of the MHC molecules I $(190.00 \% \pm$ 6.17; Fig. 3.10) and II (202.20\% \pm 3.43 ; Fig. 3.11) compared to control cells of nonimmunized mice or OVA-negative B cells of immunized mice (106.80 \% \pm 13.20 and $84.96 \%$ \pm 3.22 , respectively).

A

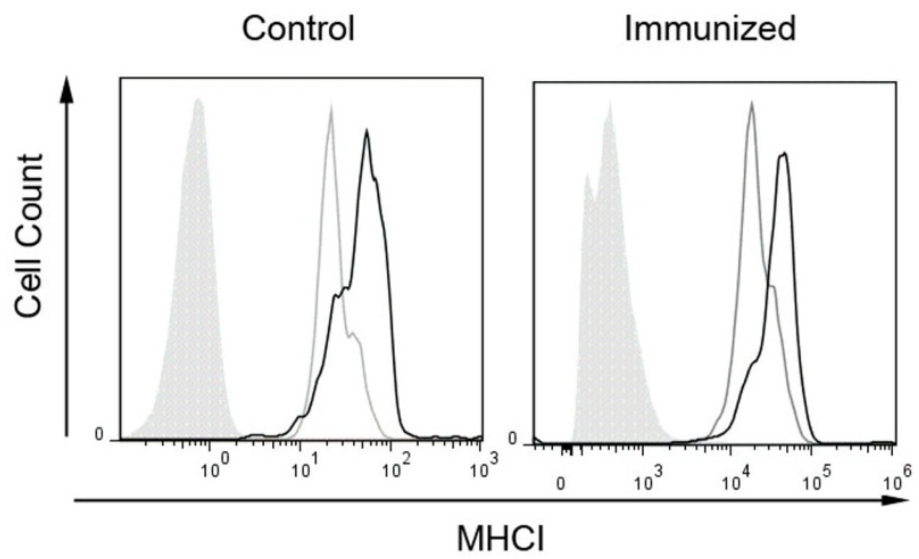

$\mathrm{B}$

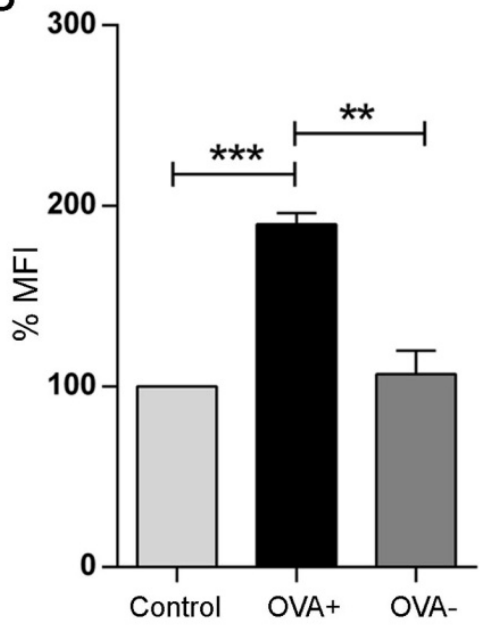

Fig. 3.10 MHC I expression in OVA-specific B cells. OVA-specific B cells from immunized mice were stained for their expression of $\mathrm{MHCI}$ and analyzed by flow cytometry. (A) Representative analyses out of 5 independent experiments are shown. OVA-specific B cells (black line) were compared to B cells of non-immunized (control) mice (left plot, grey line) and OVA-negative B cells of immunized mice (right plot, grey line). Cells that were analyzed without antibody staining served as unstained 
control (filled histogram). (B) The mean fluorescent intensity (MFI) of MHC I of OVA-specific (OVA+) and OVA-negative (OVA-) $B$ cells in immunized mice were normalized to the expression levels in $B$ cells of control mice. Bar charts show mean values \pm SEM of three independent experiments. Significant differences were calculated with ordinary one-way ANOVA are marked by an asterisk. ${ }^{* *} \mathrm{p}$ $\leq 0.01, * * * \mathrm{p} \leq 0.001$.

A

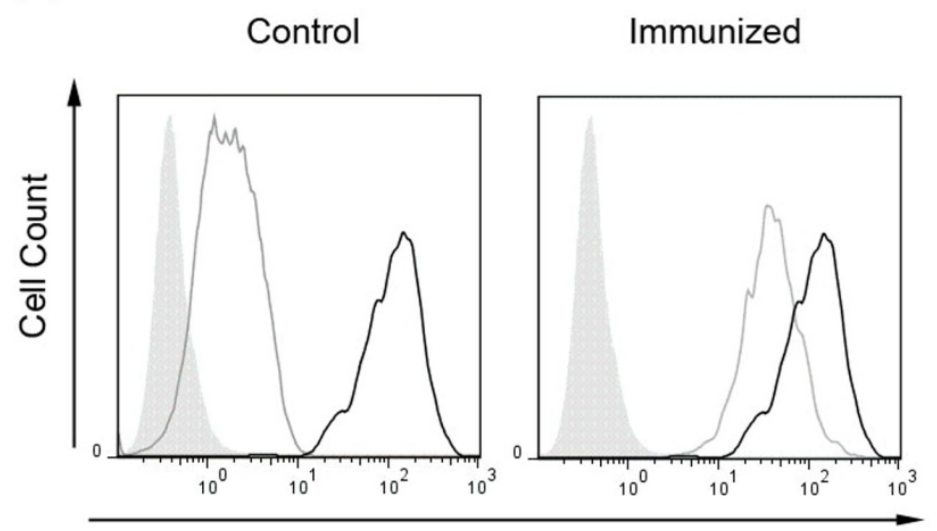

$\mathrm{IAb}$

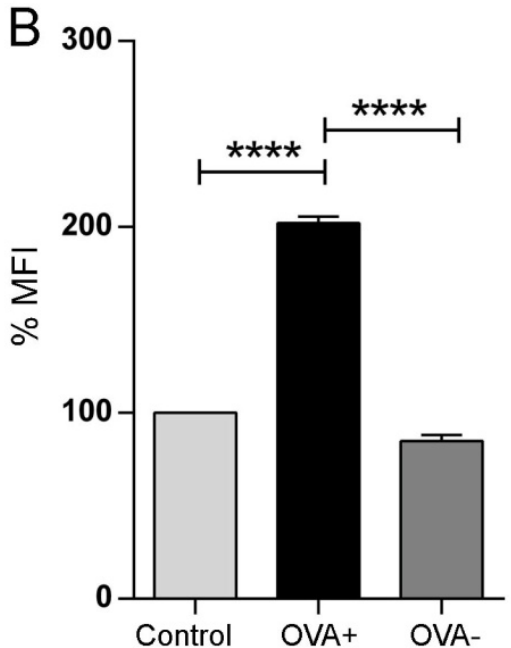

Fig. 3.11 MHC II expression in OVA-specific B cells. OVA-specific B cells from immunized mice were stained for their expression of MHC II and analyzed by flow cytometry. (A) Representative analyses out of 5 independent experiments are shown. OVA-specific B cells (black line) were compared to B cells of non-immunized (control) mice (left plot, grey line) and OVA-negative B cells of immunized mice (right plot, grey line). Cells that were analyzed without antibody staining served as unstained control (filled histogram). (B) The mean fluorescent intensity (MFI) of MHC II of OVA-specific (OVA+) and OVA-negative (OVA-) B cells in immunized mice were normalized to the expression levels in $B$ cells of control mice. Bar charts show mean values \pm SEM of three independent experiments. Significant differences were calculated with ordinary one-way ANOVA are marked by an asterisk. ${ }^{*} p$ $\leq 0.05, * * * * \mathrm{p} \leq 0.0001$.

Moreover, the expression of the costimulatory molecules CD86 was significantly higher in OVA-specific B cells $(182.60 \% \pm 15.30 ;$ Fig. 3.12$)$ than in control B cells or OVA-negative B cells (110.50\% \pm 10.49$)$. Although OVA-specific B cells also upregulated the expression of CD80 (129.90 \% \pm 49.06 ; Fig. 3.13), the differences to control B cells or OVA-negative B cells (128.6\% \pm 14.02$)$ was not significant. KLH-specific B cells showed similar upregulation of the activation markers (data not shown). 
A

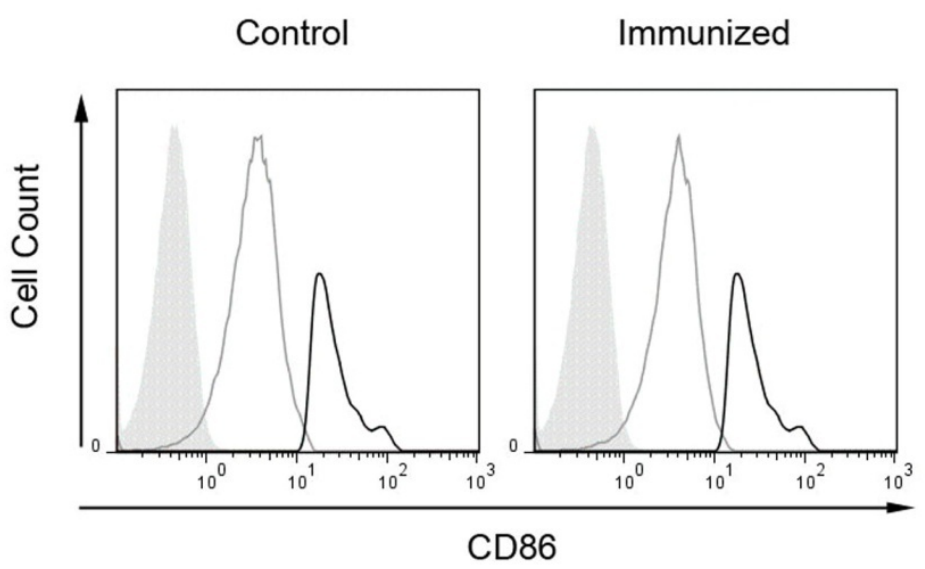

B

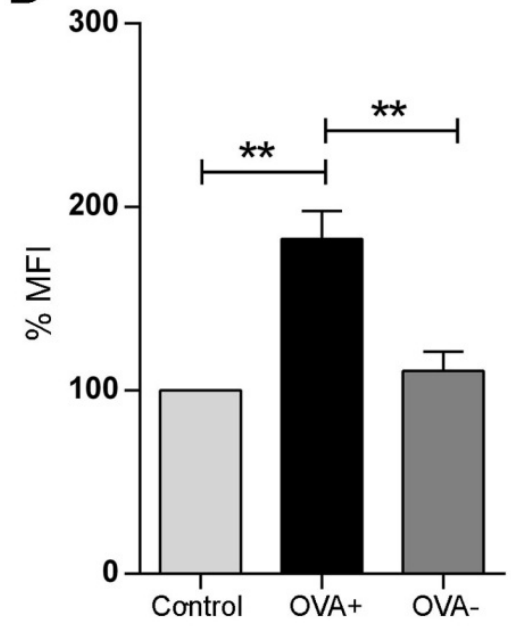

Fig. 3.12 CD86 expression in OVA-specific B cells. OVA-specific B cells from immunized mice were stained for their expression of CD86 and analyzed by flow cytometrry. (A) Representative analyses out of 5 independent experiments are shown. OVA-specific B cells (black line) were compared to B cells of non-immunized (control) mice (left plot, grey line) and OVA-negative B cells of immunized mice (right plot, grey line). Cells that were analyzed without antibody staining served as unstained control (filled histogram). (B) The mean fluorescent intensity (MFI) of CD86 of OVA-specific (OVA+) and OVA-negative (OVA-) B cells in immunized mice were normalized to the expression levels in $B$ cells of control mice. Bar charts show mean values \pm SEM of three independent experiments. Significant differences were calculated with ordinary one-way ANOVA are marked by an asterisk. ${ }^{* *} \mathrm{p}$ $\leq 0.01$.

A

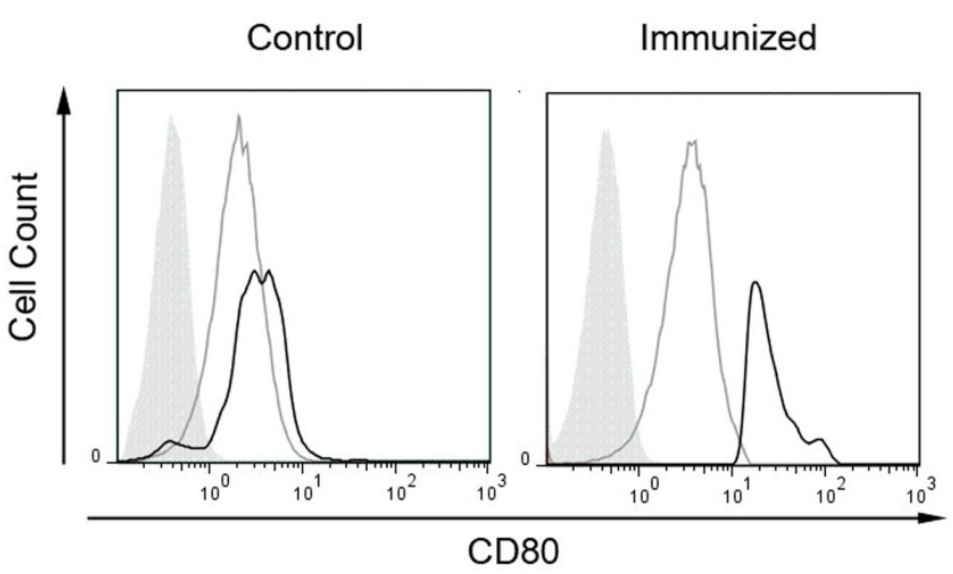

$\mathrm{B}$

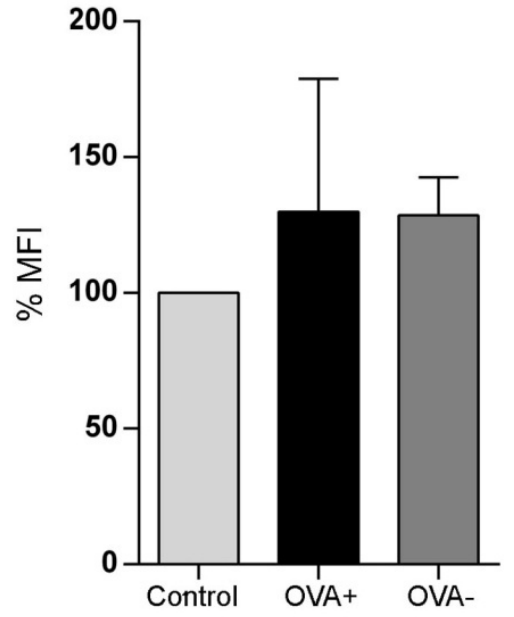

Fig. 3.13 CD80 expression in OVA-specific B cells. OVA-specific $B$ cells from immunized mice were stained for their expression of CD80 and analyzed by flow cytometry. (A) Representative analyses out of 5 independent experiments are shown. OVA-specific B cells (black line) were compared to B cells of non-immunized (control) mice (left plot, grey line) and OVA-negative B cells of immunized mice (right plot, grey line). Cells that were analyzed without antibody staining served as unstained control (filled histogram). (B) The mean fluorescent intensity (MFI) of CD80 of OVA-specific (OVA+) and OVAnegative (OVA-) B cells in immunized mice were normalized to the expression levels in $B$ cells of control mice. Bar charts show mean values \pm SEM of three independent experiments. Significant differences calculated with ordinary one-way ANOVA were not detected. 
The percentage $\mathrm{CD} 138^{+} \mathrm{B}$ cells in OVA-specific B cells varied greatly in the different mice, but overall was increased ( $7.18 \% \pm 5.07$; Fig. 3.14$)$ compared to control B cells $(1.85 \% \pm 0.50)$ and OVA-negative B cells $(2.48 \% \pm 0.73)$. However, the difference was not significant. KLHspecific B cells showed similar increase in $\mathrm{CD}_{138^{+}}$cells (data not shown).

A

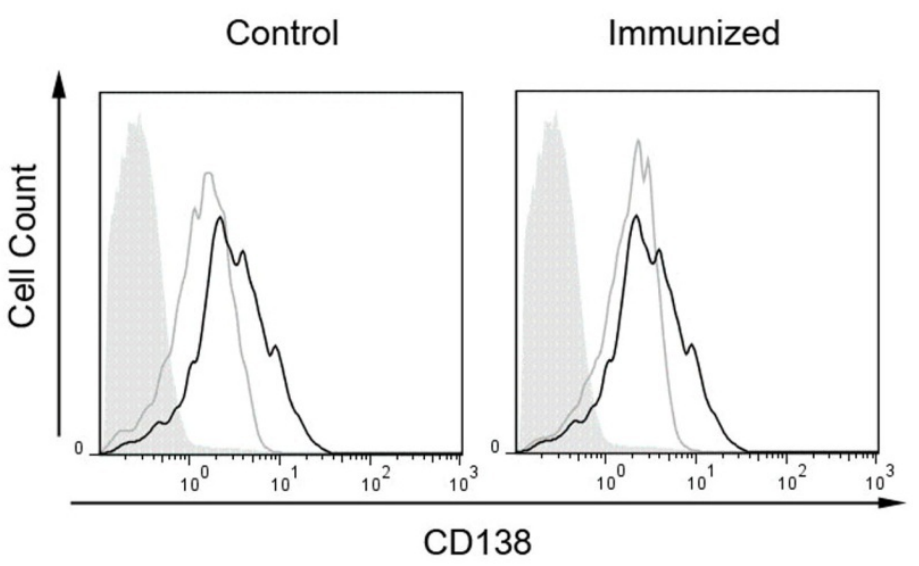

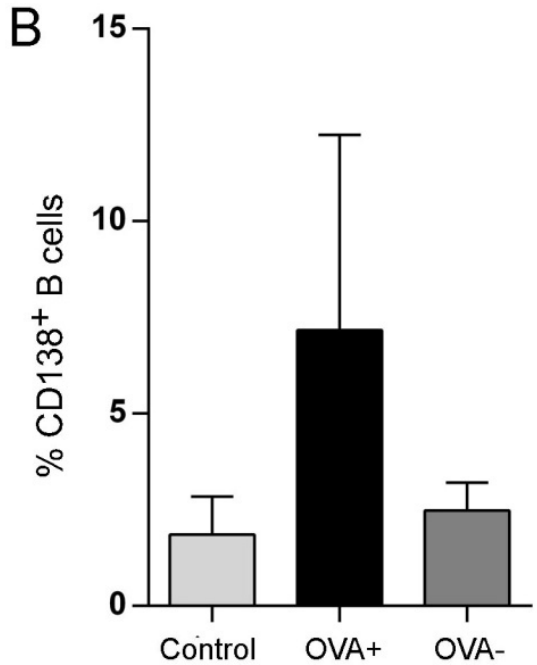

Fig. 3.14 CD138 ${ }^{+}$OVA-specific B cells. OVA-specific B cells from immunized mice were stained for their expression of CD138 and analyzed by flow cytometry. (A) Representative analyses out of 5 independent experiments are shown. OVA-specific B cells (black line) were compared to B cells of non-immunized (control) mice (left plot, grey line) and OVA-negative B cells of immunized mice (right plot, grey line). Cells that were analyzed without antibody staining served as unstained control (filled histogram). (B) The percentage of $\mathrm{CD} 138^{+} \mathrm{B}$ cells among OVA-specific (OVA+), OVA-negative (OVA-) or $B$ cells of control mice are shown. Bar charts show mean values \pm SEM of three independent experiments. Significant differences calculated with ordinary one-way ANOVA were not detected.

These data clearly indicate a class-switched, activated phenotype of the isolated B cells as expected for antigen-specific B cells of recently immunized mice (Good-Jacobson and Shlomchik 2010).

\subsubsection{Efficient Purification of Human Antigen-Specific B Cells Depends on Initial HBV- Specific B Cell Frequency}

Hepatitis-B-Virus (HBV) antigen was chosen as model antigen for the isolation of antigenspecific B cells in a human setting. Blood donors vaccinated against HBV are easily accessible, since by 201279 \% of children in 183 World Health Organization (WHO) member states had received HBV vaccination (WHO 2015). Nevertheless, non-vaccinated donors are still available, since HBV vaccination only became a standard treatment in children in 1982.

The percentage of $\mathrm{HBV}$-specific $\mathrm{B}$ cells among all $\mathrm{CD} 19^{+} \mathrm{CD} 20^{+} \mathrm{B}$ cells was determined by labeling with antigen tetramers and FACS analyses (Fig. 3.1). For identification of the positive 
population, gates were adjusted to negative control donors. The percentage of HBV-specific B cells varied greatly from 0.60 to $2.50 \%$ in vaccinated donors normalized to control donors (Fig. 3.15 middle plot and Fig. 3.16, lower left plot, respectively).

HBV-specific B cells could be enriched from PBMCs by positive selection via antigen tetramers. The efficiency of the enrichment strongly depended on the grade of biotinylation of the antigen as well as the initial frequency of HBV-specific B cells. Therefore, the purity of the enriched cells varied between 13.57 and $34.35 \%$ (Fig. 3.15, right plot and 3.16, lower right plot, respectively). The percentage of B cells that were stained unspecifically for HBV increased when purifying control B cells by antigen tetramers (Fig. 3.16, upper right plot). However, the purity of the HBV-specific population was higher and therefore specific.
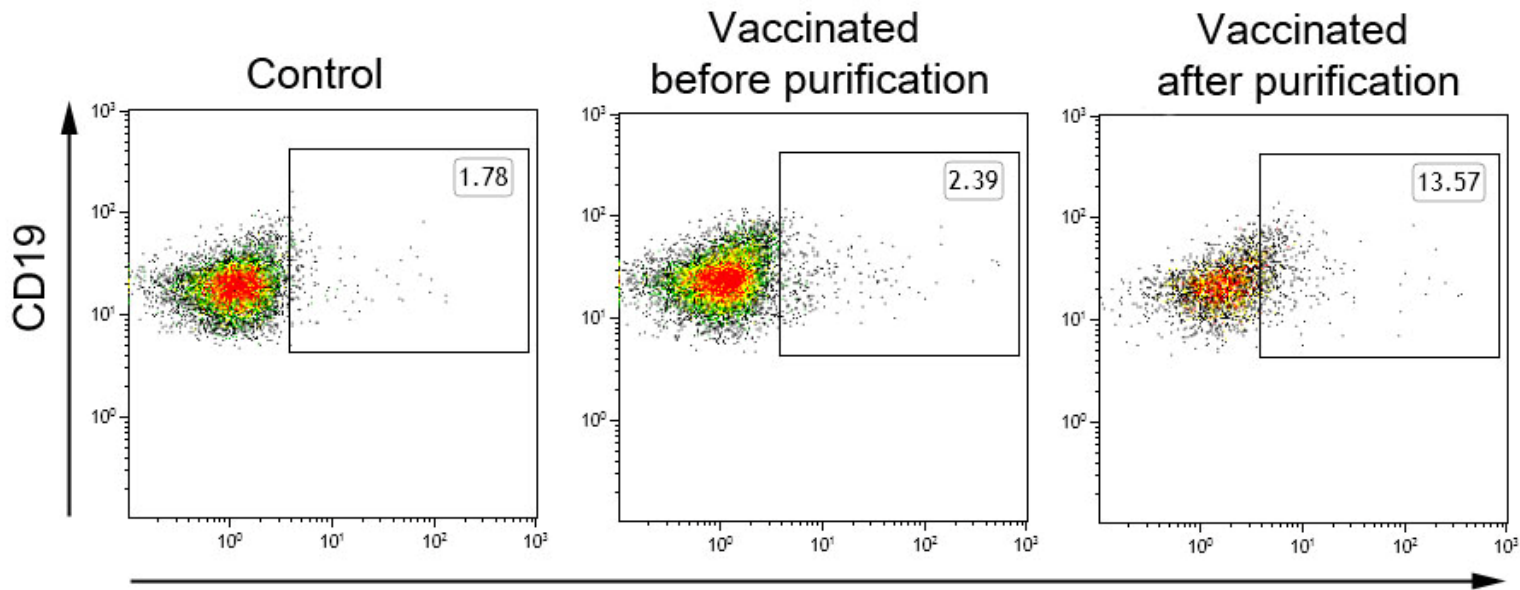

HBV

Fig. 3.15 Purification of HBV-specific B cells from donors with low frequencies. PBMCs from donors were stained for HBV-specific B cells among the $\mathrm{CD} 19^{+} \mathrm{CD} 2 \mathrm{O}^{+} \mathrm{B}$ cell population with HBV-Biotin tetramers. Representative flow cytometry analyses out of 5 independent experiments with nonvaccinated donors (control, left plot) or vaccinated donors (middle and right plot) are shown. HBVspecific $B$ cells of vaccinated donors were purified by positive selection with HBV-Biotin tetramers (right plot). Numbers indicate the percentage of $\mathrm{HBV}^{+} \mathrm{B}$ cells. 


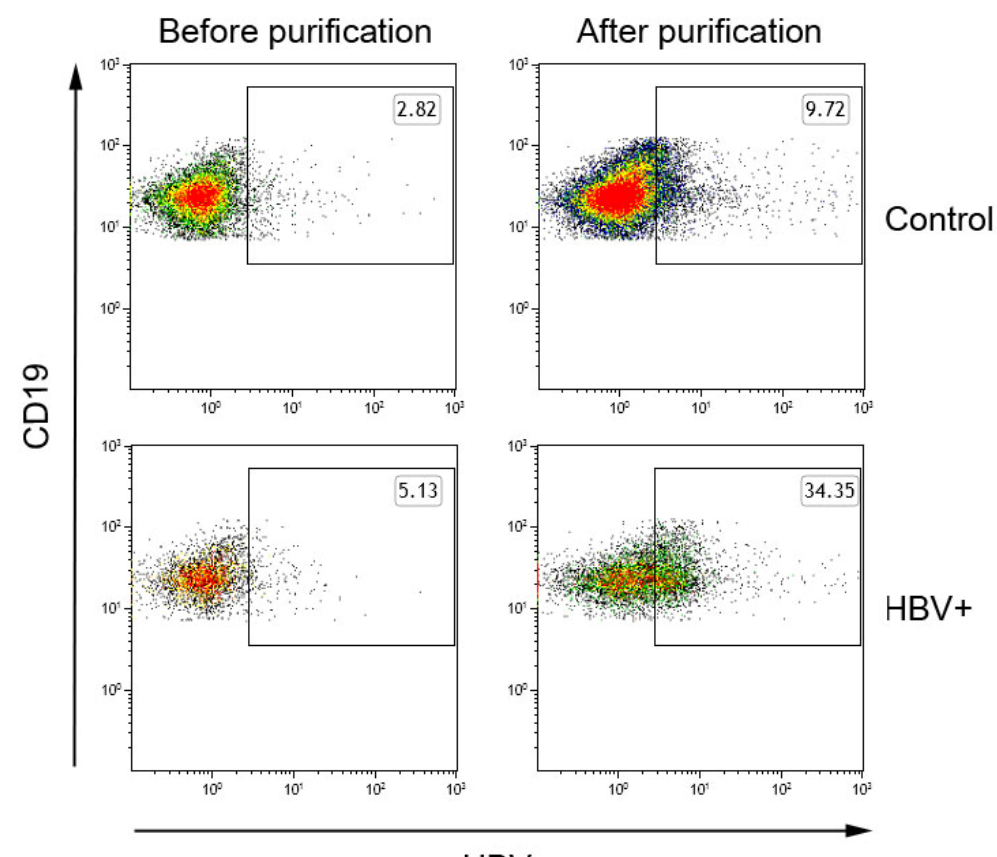

HBV
Fig. 3.16 Purification of HBVspecific $B$ cells from donors with high frequencies. PBMCs from donors were stained for HBV-specific B cells among the $\mathrm{CD}_{19} \mathrm{CD}^{+} \mathrm{O}^{+} \mathrm{B}$ cell population with HBV-Biotin tetramers. Representative flow cytometry analyses out of five independent experiments with nonvaccinated donors (control, upper plots) or vaccinated donors (lower plots) are shown. PBMCs were either stained immediately (left plots) or were purified by positive selection with HBVBiotin tetramers (right plots). Numbers indicate the percentage of $\mathrm{HBV}^{+} \mathrm{B}$ cells.

The overall purity of $\mathrm{CD} 19^{+} \mathrm{CD} 20^{+} \mathrm{B}$ cells varied between $4.24 \%$ and $23.31 \%$ for nonvaccinated non-purified donors and $9.22 \%$ and $20.53 \%$ for vaccinated antigen-enriched donors (Fig. 3.17).

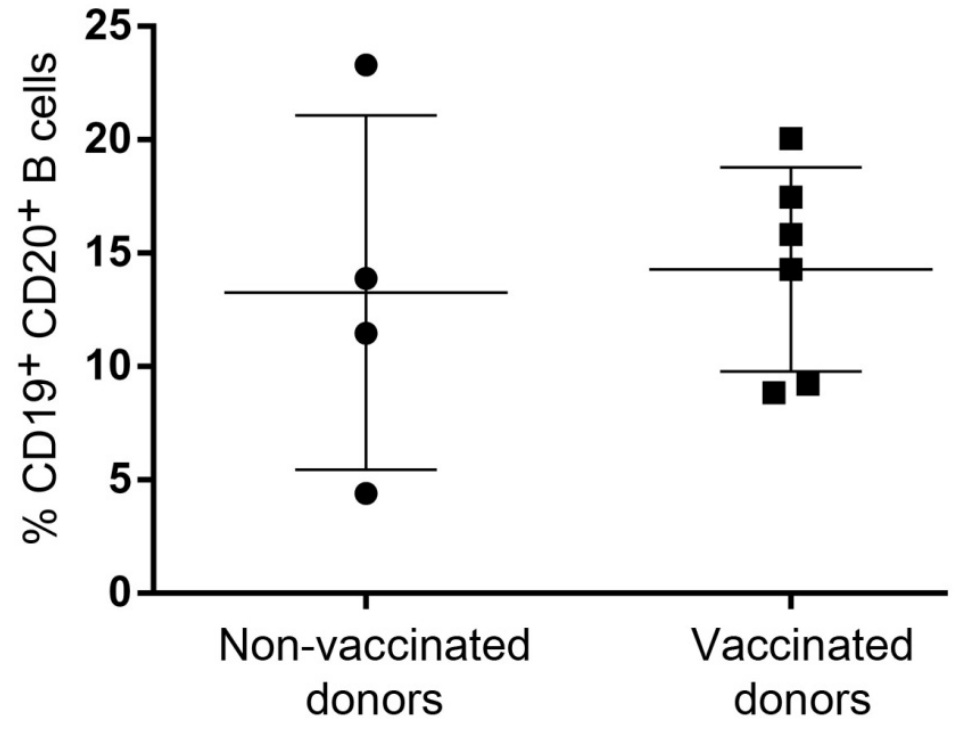

Fig. 3.17 B cell purity of HBVenriched $B$ cells. PBMCs of vaccinated and non-vaccinated donors were purified by positive selection with HBVBiotin tetramers and stained for $\mathrm{CD} 19^{+} \mathrm{CD} 20^{+} \mathrm{B}$ cells. Results of at least 4 donors are shown.

\subsubsection{Human Antigen-Specific B Cells Show a Class-Switched Phenotype}

Analogous to murine antigen-specific B cells, the isolated HBV-specific B cells were characterized by FACS analysis for several surface markers defining their activation status and developmental state. Bar charts represent results of at least three independent 
experiments. Expression levels of B cells of vaccinated donors were normalized to the expression levels of B cells of non-vaccinated donors.

The expression of the B cell marker CD20 was significantly upregulated in HBV-specific CD19 ${ }^{+}$ CD20 ${ }^{+}$B cells $(222.1 \% \pm 21.43)$ compared to control B cells of non-vaccinated donors or HBV-negative B cells of vaccinated donors (122.0 \% \pm 8.69 ; Fig. 3.18). The same was observed for the B cell marker CD19, which was significantly upregulated in HBV-specific B cells $(174.0 \% \pm 9.70)$ compared to control B cells and HBV-negative B cells $(107.5 \% \pm 2.70$; Fig. 3.19).

A

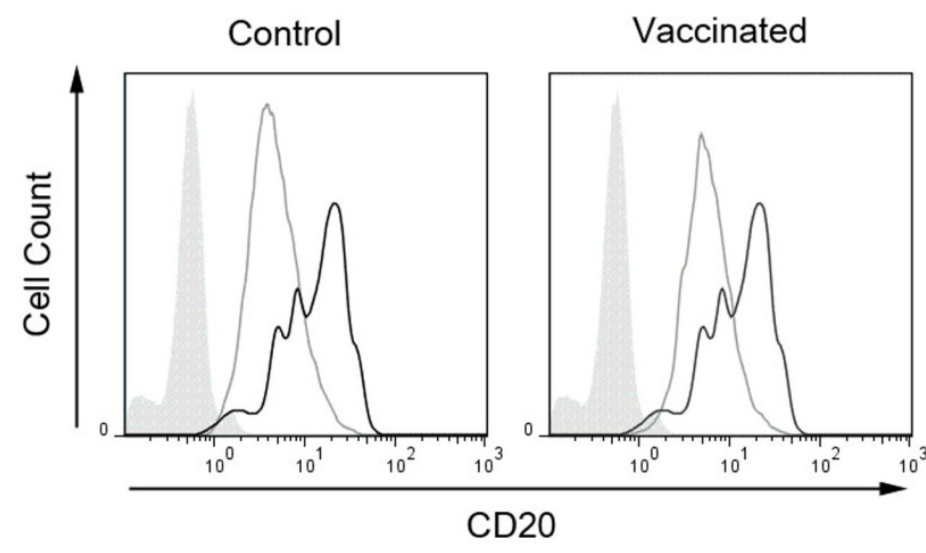

$\mathrm{B}$

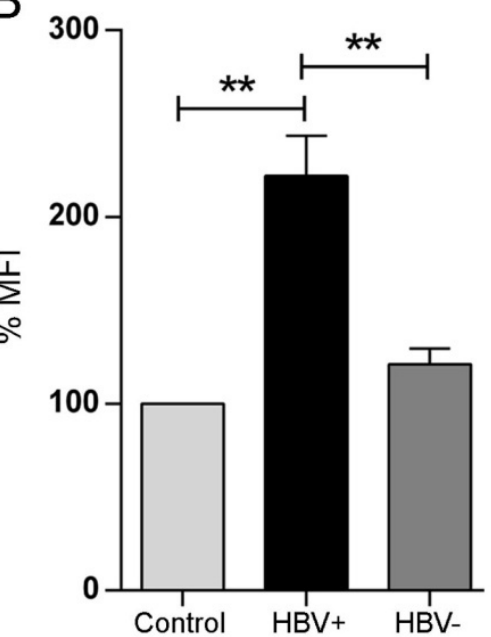

Fig. 3.18 CD20 expression in HBV-specific B cells. HBV-specific B cells from vaccinated donors were stained for their expression of $\mathrm{CD} 20$ and analyzed by flow cytometry. (A) Representative analyses out of 3 independent donors are shown. HBV-specific B cells (black line) were compared to B cells of a non-vaccinated (control) donor (left plot, grey line) and HBV-negative B cells of the same donor (right plot, grey line). Cells that were analyzed without antibody staining served as unstained control (filled histogram). (B) The mean fluorescent intensity (MFI) of CD2O of HBV-specific (HBV+) and HBVnegative (HBV-) B cells in vaccinated donors were normalized to the expression levels in $B$ cells of control donors. Bar charts show mean values \pm SEM of three independent experiments. Significant differences were calculated with ordinary one-way ANOVA are marked by an asterisk. ${ }^{* *} p \leq 0.01$. 
A

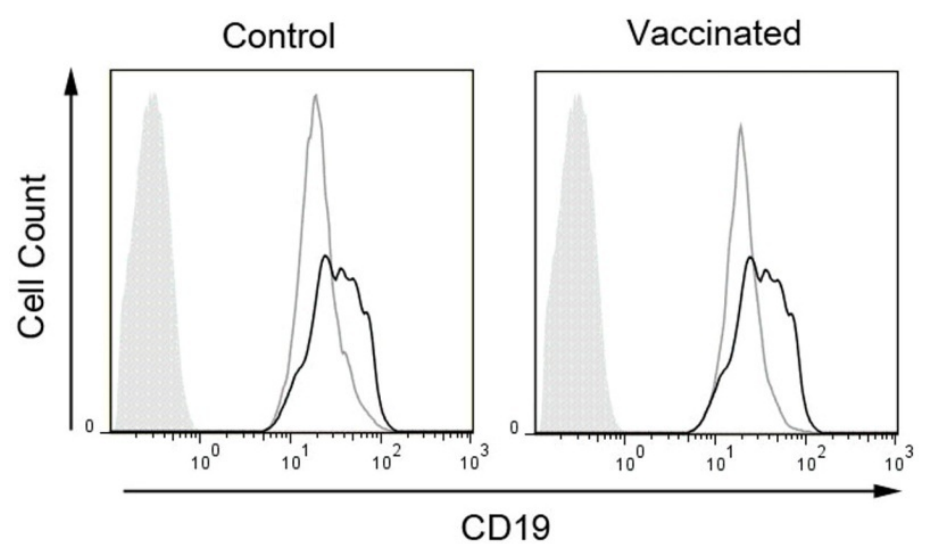

B

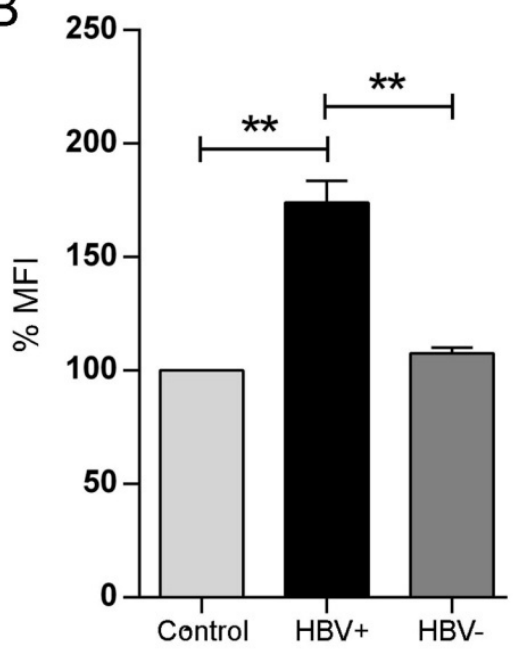

Fig. 3.19 CD19 expression in HBV-specific B cells. HBV-specific B cells from vaccinated donors were stained for their expression of CD19 and analyzed by flow cytometry. (A) Representative analyses out of 3 independent donors are shown. HBV-specific B cells (black line) were compared to B cells of a non-vaccinated (control) donor (left plot, grey line) and HBV-negative B cells of the same donor (right plot, grey line). Cells that were analyzed without antibody staining served as unstained control (filled histogram). (B) The mean fluorescent intensity (MFI) of CD19 of HBV-specific (HBV+) and HBVnegative (HBV-) B cells in vaccinated donors were normalized to the expression levels in $B$ cells of control donors. Bar charts show mean values \pm SEM of three independent experiments. Significant differences were calculated with ordinary one-way ANOVA are marked by an asterisk. ${ }^{* *} p \leq 0.01$.

Interestingly, in contrast to murine antigen-specific B cells, the percentage of $\operatorname{lgD}^{+}$and $\operatorname{lgM} M^{+}$ HBV-specific $\mathrm{CD} 19^{+} \mathrm{CD} 20^{+} \mathrm{B}$ cells was almost as high $(77.67 \% \pm 7.69$ and $83.26 \% \pm 8.83$, respectively) as in control B cells (85.14 \% \pm 8.11 and $76.42 \% \pm 6.87$, respectively) and HBVnegative $B$ cells $(79.07 \% \pm 6.86$ and $77.80 \% \pm 5.71$, respectively; Fig. 3.20 and Fig. 3.21, respectively). $\lg \mathrm{D}^{+} \mathrm{B}$ cells could be subdivided into two types by additional staining for CD27. Classical IgD ${ }^{+}$CD27 naïve B cells were significantly decreased in HBV-specific B cells $(38.78 \%$ $\pm 10.07)$ compared to control $(73.48 \% \pm 21.58)$ and HBV-negative B cells $(59.75 \% \pm 8.01$, Fig. 3.22). $\lg D^{+} C D 27^{+} B$ cells were significantly increased in HBV-specific B cells $(45.89 \% \pm$ 12.67) compared to control $(12.59 \% \pm 11.32)$ and HBV-negative B cells $(23.52 \% \pm 9.04)$. The percentages of classical IgD $\mathrm{CD}^{-} 7^{+}$memory $\mathrm{B}$ cells were more or less equal in the different populations. As expected, significantly more HBV-specific B cells were positive for IgG1 (34.89\% $\%$ 5.66) compared to control B cells $(6.09 \% \pm 1.14)$ or HBV-negative B cells $(3.32 \% \pm$ 0.01; Fig. 3.23). 
A

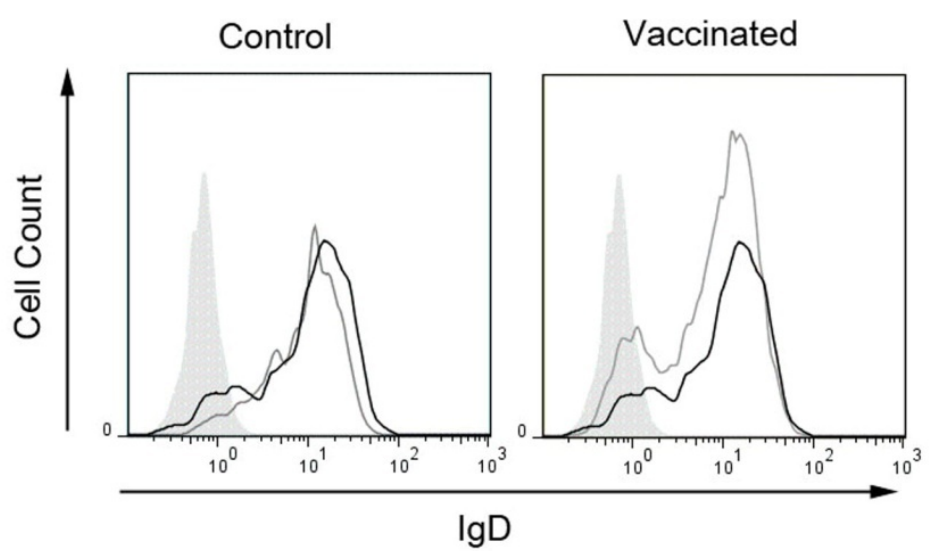

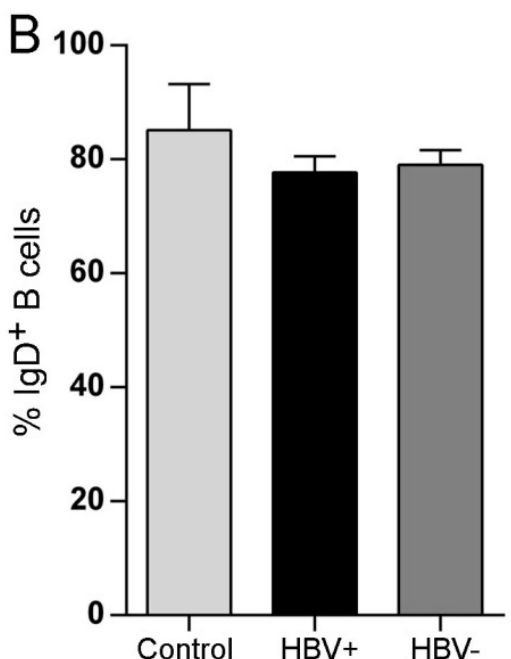

Fig. 3.20 IgD ${ }^{+}$HBV-specific B cells. HBV-specific B cells from vaccinated donors were stained for their expression of IgD and analyzed by flow cytometry. (A) Representative analyses out of 3 independent donors are shown. HBV-specific B cells (black line) were compared to B cells of a non-vaccinated (control) donor (left plot, grey line) and HBV-negative B cells of the same donor (right plot, grey line). Cells that were analyzed without antibody staining served as unstained control (filled histogram). (B) The percentage of IgD ${ }^{+}$B cells among HBV-specific ( $\mathrm{HBV}+$ ), HBV-negative (HBV-) B cells in vaccinated donors and $B$ cells of control donors are shown. Bar charts show mean values \pm SEM of three independent experiments. Significant differences calculated with ordinary one-way ANOVA were not detected.

A

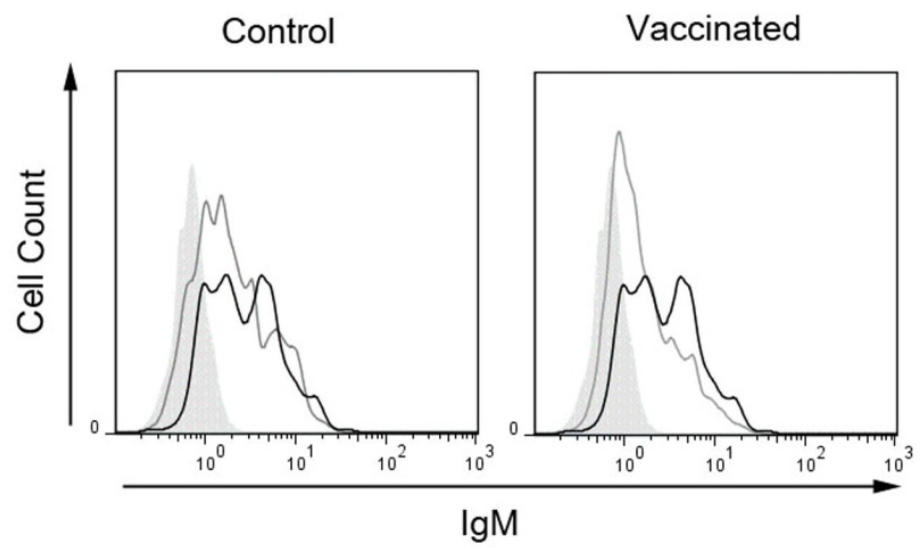

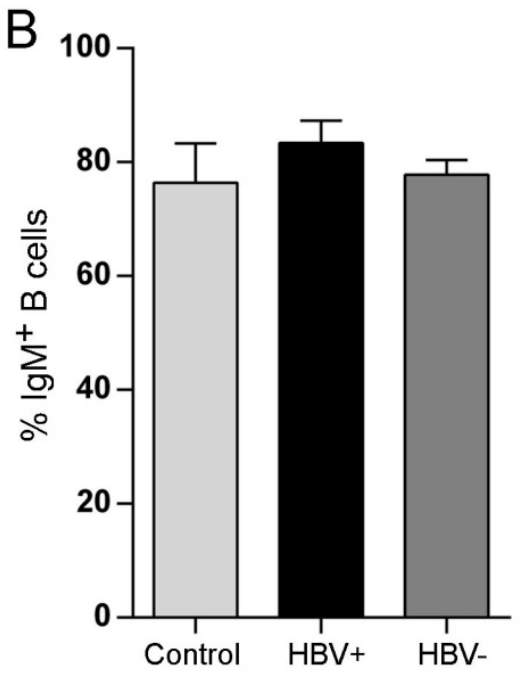

Fig. 3.21 IgM ${ }^{+}$HBV-specific B cells. HBV-specific B cells from vaccinated donors were stained for their expression of IgM and analyzed by flow cytometry. (A) Representative analyses out of 3 independent donors are shown. HBV-specific B cells (black line) were compared to B cells of a non-vaccinated (control) donor (left plot, grey line) and HBV-negative B cells of the same donor (right plot, grey line). Cells that were analyzed without antibody staining served as unstained control (filled histogram). (B) The percentage of $\mathrm{IgM}^{+} \mathrm{B}$ cells among HBV-specific (HBV+), HBV-negative (HBV-) B cells in vaccinated donors and $B$ cells of control donors are shown. Bar charts show mean values \pm SEM of three independent experiments. Significant differences calculated with ordinary one-way ANOVA were not detected. 


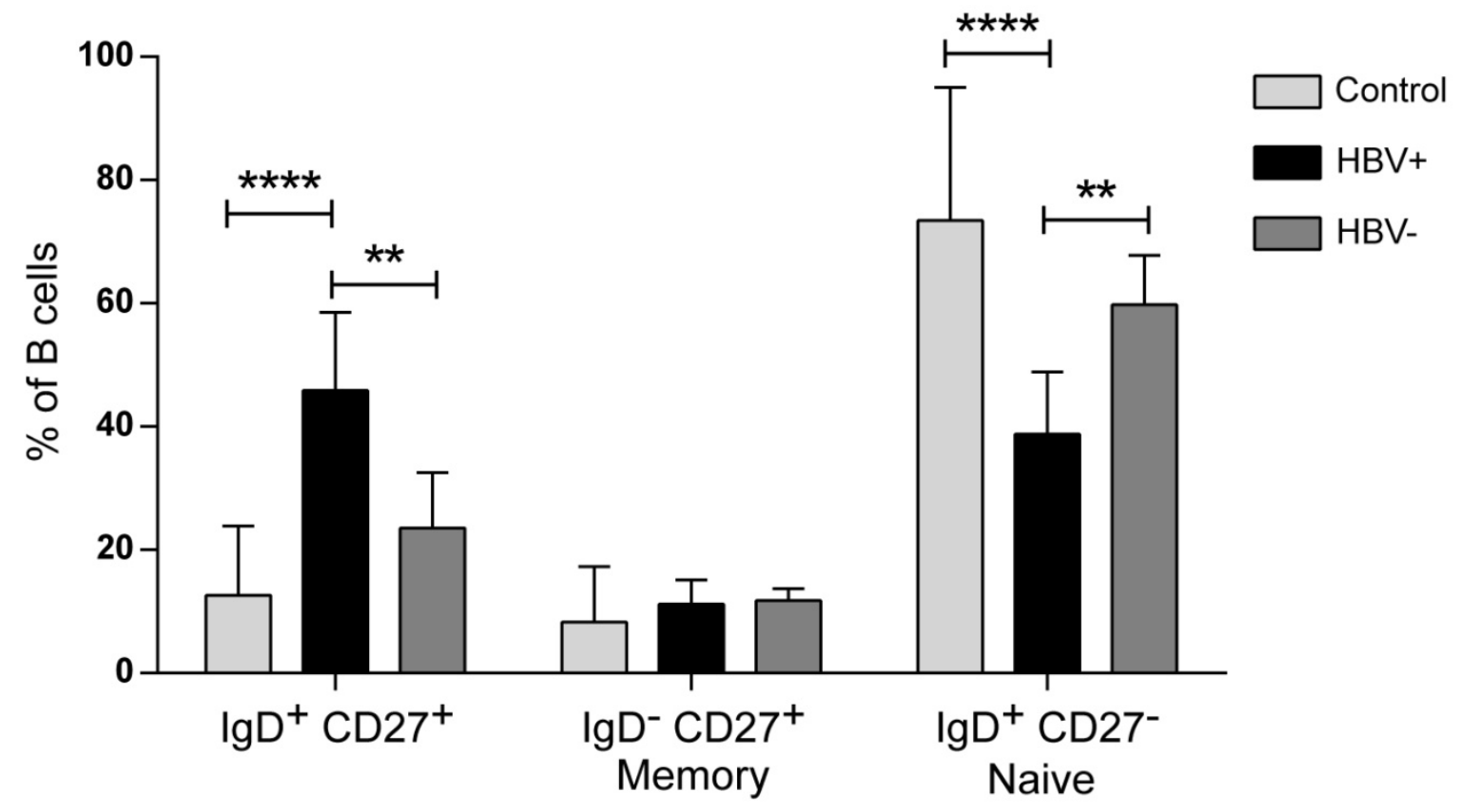

Fig. 3.22 IgD CD27 expression in HBV-specific B cells. HBV-specific B cells from vaccinated donors were stained for their expression of IgD and CD27 analyzed by flow cytometry. The percentages of the different IgD and CD27 subtypes of HBV-specific (HBV+), HBV-negative (HBV-) and control B cells are shown. Bar charts show mean values \pm SD of five independent experiments. Significant differences were calculated with two-way ANOVA are marked by an asterisk. ${ }^{* *} p \leq 0.01, * * * * p \leq$ 0.0001 .

A

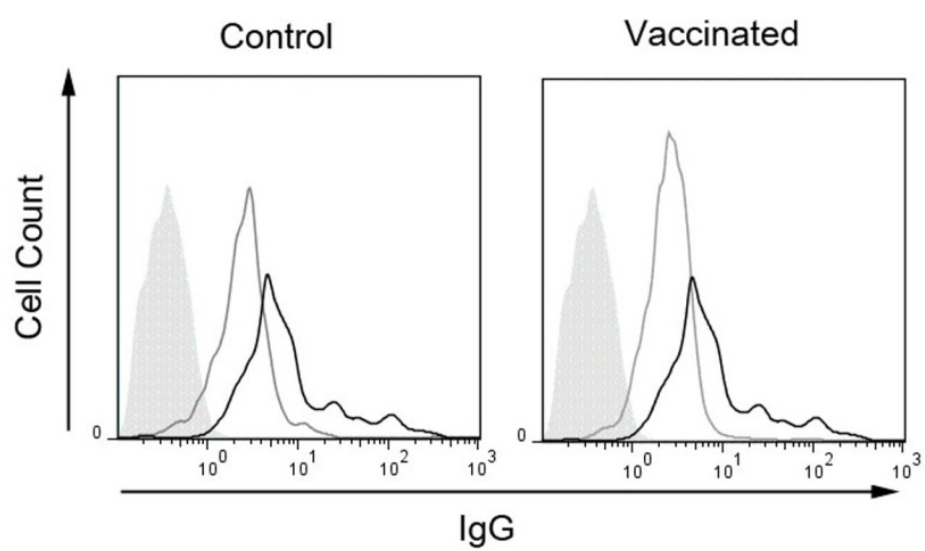

$\mathrm{B}$

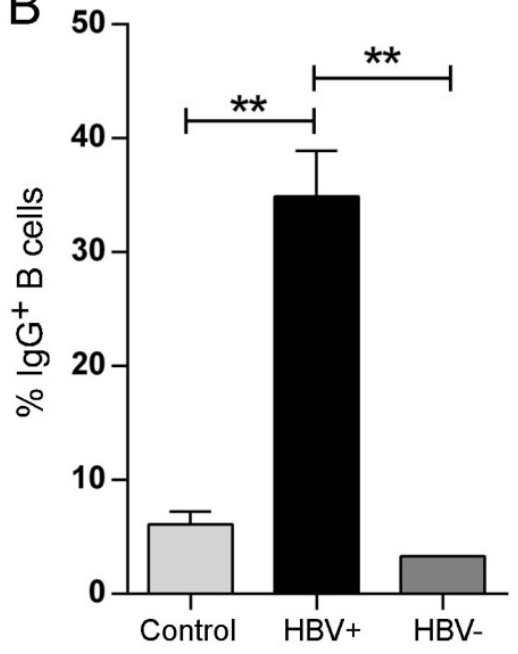

Fig. 3.23 IgG1 ${ }^{+}$HBV-specific B cells. HBV-specific B cells from vaccinated donors were stained for their expression of IgG1 and analyzed by flow cytometry. (A) Representative analyses out of 3 independent donors are shown. HBV-specific B cells (black line) were compared to B cells of a nonvaccinated (control) donor (left plot, grey line) and HBV-negative B cells of the same donor (right plot, grey line). Cells that were analyzed without antibody staining served as unstained control (filled histogram). (B) The percentage of IgG1 ${ }^{+}$B cells among HBV-specific (HBV+), HBV-negative (HBV-) B cells in vaccinated donors and $B$ cells of control donors are shown. Bar charts show mean values \pm SEM of three independent experiments. Significant differences were calculated with ordinary oneway ANOVA are marked by an asterisk. ${ }^{* *} p \leq 0.01$. 
HBV-specific B cells show significantly higher expression of the MHC molecule II HLA-DR (169.7 \% \pm 24.75 ; Fig. 3.24) compared to control B cells. The difference to HBV-negative B cells of the same donor was not significant $(142.8 \% \pm 12.10)$.

A

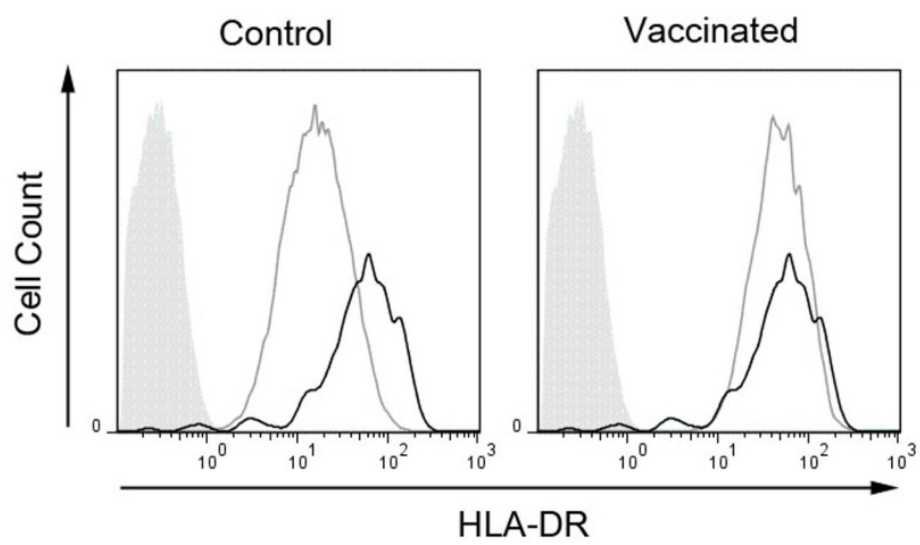

B

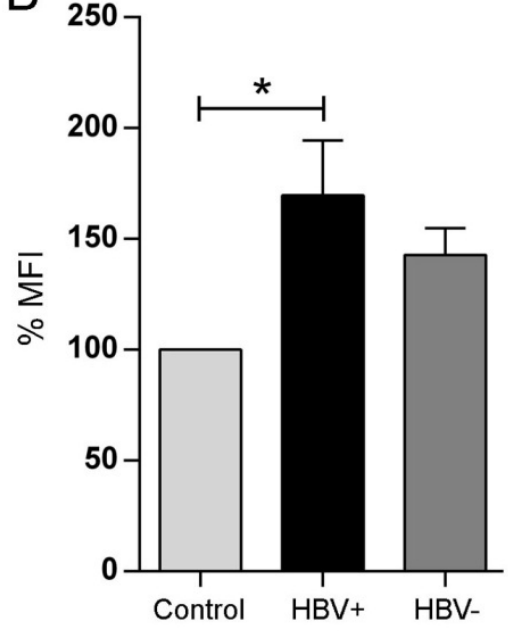

Fig. 3.24 MHC II expression in HBV-specific B cells. HBV-specific B cells from vaccinated donors were stained for their expression of MHC II and analyzed by flow cytometry. (A) Representative analyses out of 3 independent donors are shown. HBV-specific B cells (black line) were compared to B cells of a non-vaccinated (control) donor (left plot, grey line) and HBV-negative B cells of the same donor (right plot, grey line). Cells that were analyzed without antibody staining served as unstained control (filled histogram). (B) The mean fluorescent intensity (MFI) of MHC II of HBV-specific (HBV+) and HBV-negative (HBV-) B cells in vaccinated donors were normalized to the expression levels in B cells of control donors. Bar charts show mean values \pm SEM of three independent experiments. Significant differences were calculated with ordinary one-way ANOVA are marked by an asterisk. ${ }^{*} p \leq 0.05$.

Moreover, the expression of the costimulatory molecule CD86 was significantly higher in HBV-specific B cells (133.3 \% \pm 8.33 ; Fig. 3.25 ) than in control B cells or HBV-negative B cells (94.44 \% \pm 3.67$)$. Although HBV-specific B cells also upregulated the expression of CD80 (230.1 \% \pm 59.58; Fig. 3.26), the differences to control B cells or HBV-negative B cells (152.4 $\% \pm 74.68$ ) was not significant. 
A

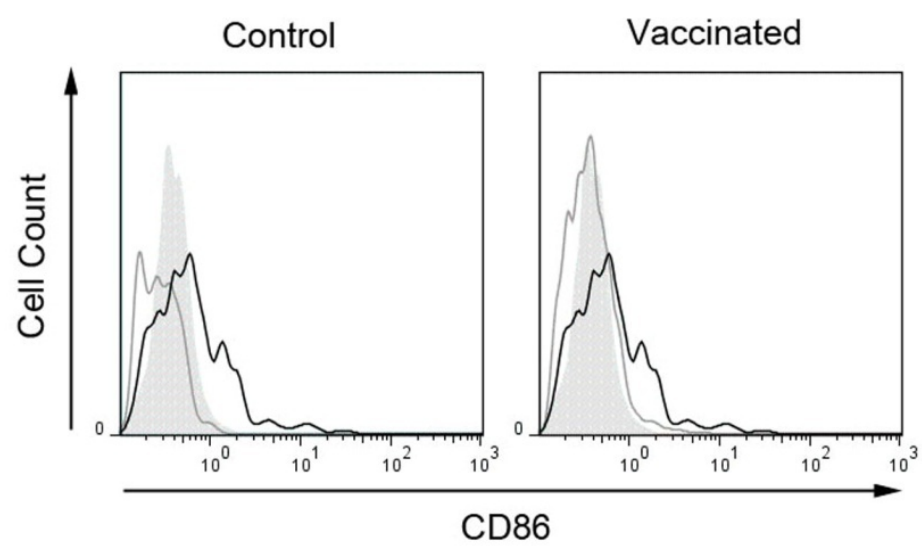

B

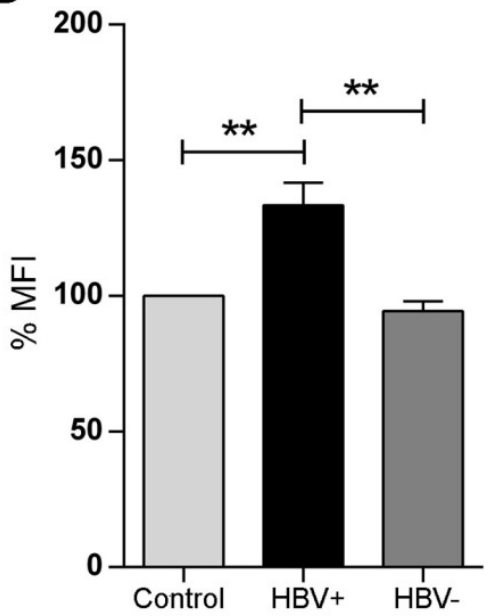

Fig. 3.25 CD86 expression in HBV-specific B cells. HBV-specific B cells from vaccinated donors were stained for their expression of CD86 and analyzed by flow cytometry. (A) Representative analyses out of 3 independent donors are shown. HBV-specific B cells (black line) were compared to B cells of a non-vaccinated (control) donor (left plot, grey line) and HBV-negative B cells of the same donor (right plot, grey line). Cells that were analyzed without antibody staining served as unstained control (filled histogram). (B) The mean fluorescent intensity (MFI) of CD86 of HBV-specific (HBV+) and HBVnegative (HBV-) B cells in vaccinated donors were normalized to the expression levels in $B$ cells of control donors. Bar charts show mean values \pm SEM of three independent experiments. Significant differences were calculated with ordinary one-way ANOVA are marked by an asterisk. ${ }^{* *} p \leq 0.01$.

A

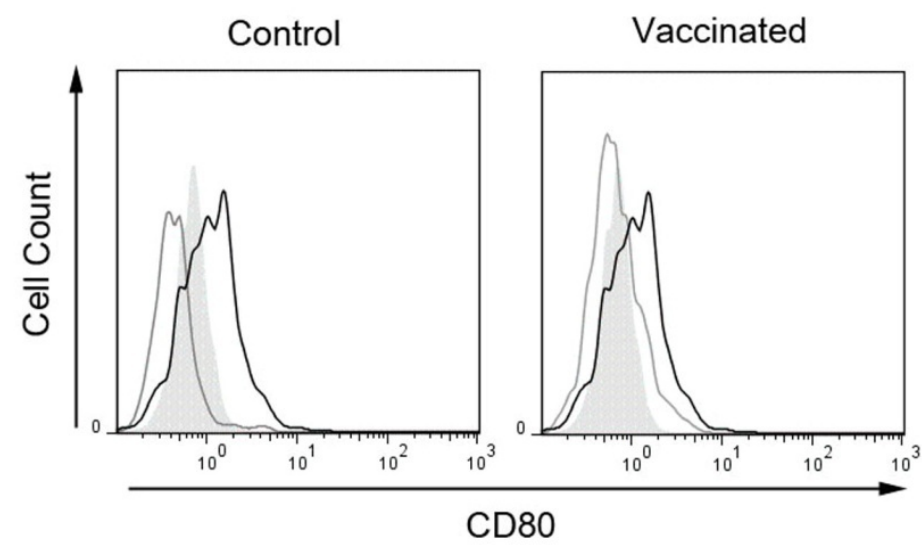

B

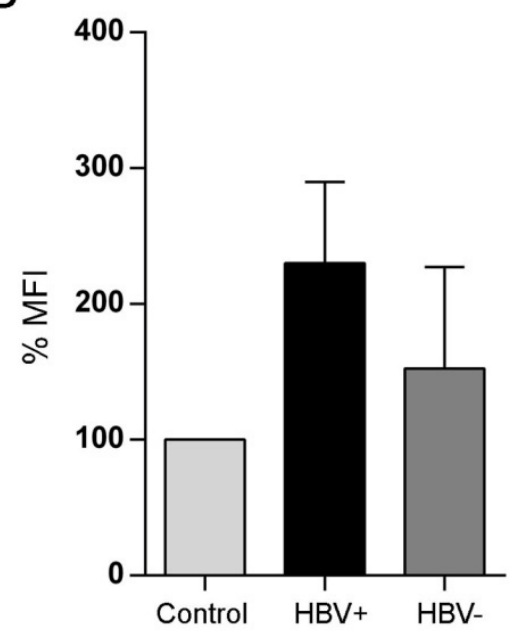

Fig. 3.26 CD80 expression in HBV-specific B cells. HBV-specific B cells from vaccinated donors were stained for their expression of CD80 and analyzed by flow cytometry. (A) Representative analyses out of 3 independent donors are shown. HBV-specific B cells (black line) were compared to B cells of a non-vaccinated (control) donor (left plot, grey line) and HBV-negative B cells of the same donor (right plot, grey line). Cells that were analyzed without antibody staining served as unstained control (filled histogram). (B) The mean fluorescent intensity (MFI) of CD80 of HBV-specific (HBV+) and HBVnegative (HBV-) B cells in vaccinated donors were normalized to the expression levels in $B$ cells of control donors. Bar charts show mean values \pm SEM of three independent experiments. Significant differences calculated with ordinary one-way ANOVA were not detected. 
Interestingly, the percentage of $\mathrm{CD} 138^{+} \mathrm{CD} 20^{+} \mathrm{CD} 19^{+}$was increased in HBV-specific B cells (16.57 \% \pm 1.7 ; Fig. 3.27) compared to control B cells $(8.41 \% \pm 1.3)$ and HBV-negative B cells $(6.77 \% \pm 0.7)$.

A

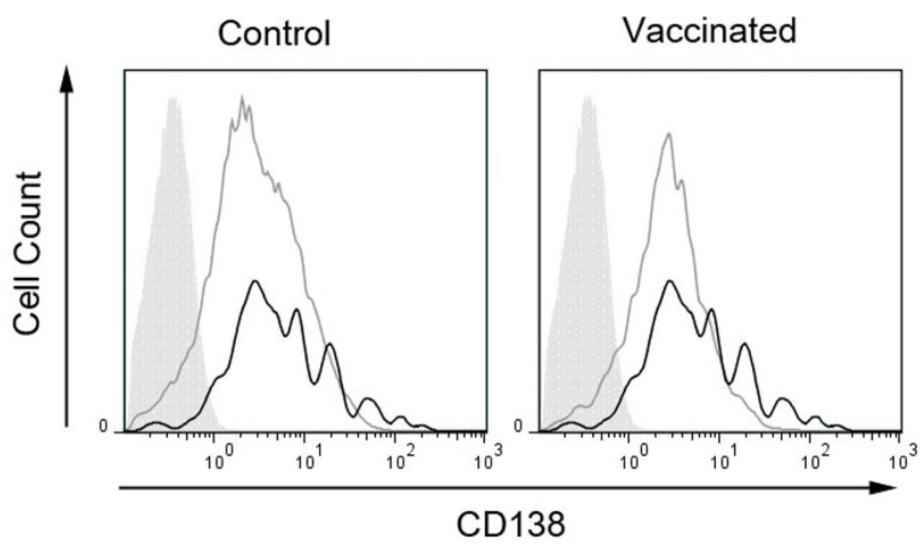

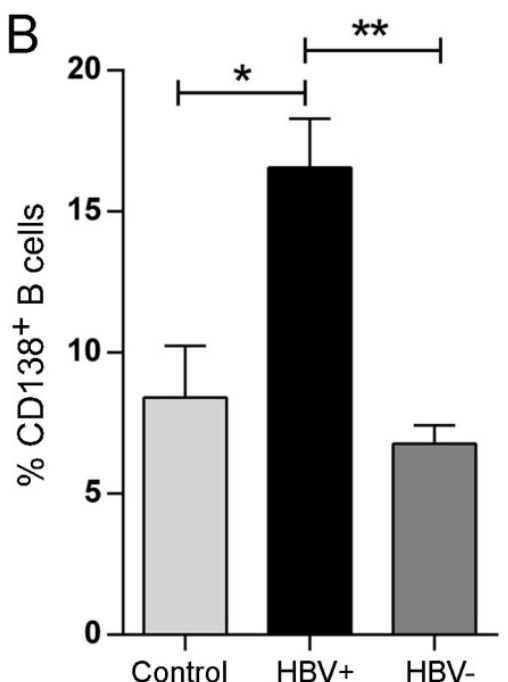

Fig. 3.27 CD138 ${ }^{+}$HBV-specific B cells. HBV-specific B cells from vaccinated donors were stained for their expression of CD138 and analyzed by flow cytometry. (A) Representative analyses out of 3 independent donors are shown. HBV-specific B cells (black line) were compared to B cells of a nonvaccinated (control) donor (left plot, grey line) and HBV-negative B cells of the same donor (right plot, grey line). Cells that were analyzed without antibody staining served as unstained control (filled histogram). (B) The percentage of CD138 ${ }^{+}$B cells among HBV-specific (HBV+), HBV-negative (HBV-) B cells in vaccinated donors and $B$ cells of control donors are shown. Bar charts show mean values \pm SEM of three independent experiments. Significant differences were calculated with ordinary oneway ANOVA are marked by an asterisk. ${ }^{*} p \leq 0.05,{ }^{* *} p \leq 0.01$.

\subsection{Stimulation with the CD40 Ligand Activates Antigen-Specific B Cells}

Through stimulation with the CD40L and IL-4, B cells upregulate costimulatory and MHC molecules and develop into highly efficient APCs (Schultze et al. 1997, Liebig et al. 2010). This in vitro culture system is established for human and murine polyclonal B cells and leads to more than $90 \%$ pure $C D 40 B$ cell cultures after 14 days. In order to use antigen-specific B cells as antigen-presenting cells for cancer immunotherapy, the purified cells were cultivated in the CD40 culture system for up to 14 days.

\subsubsection{Murine Antigen-Specific B Cells Show a Proliferative Disadvantage in the CD40L}

\section{System}

OVA-specific B cells were isolated from spleens of immunized C57BL/6 mice and enriched by positive selection. The enriched OVA-specific B cells ( $80 \%$ B cell purity and $>60 \%$ OVA purity) were cultivated on murine CD40L feeder cells (tmuCD40L HeLa cells). B cells were 
harvested on day 3, 7, 11 and 14 and recultivated on fresh feeder cells. The absolute increase of cells in culture was determined (Fig. 3.28) and pictures (Fig. 3.29) as wells as cells for Pappenheim staining (Fig. 3.30) were taken on day 7, 11 and 14. CD19 $\mathrm{B}$ cells isolated from non-immunized mice (> $90 \%$ pure) served as controls.

The number of cells in OVA-cultures decreased from day 0 to 7 , while the control cultures stayed stable (Fig. 3.28). However, from day 7 to day 11 both cultures highly proliferated resulting in a 1.3 fold expansion of initial cultures on day 14.

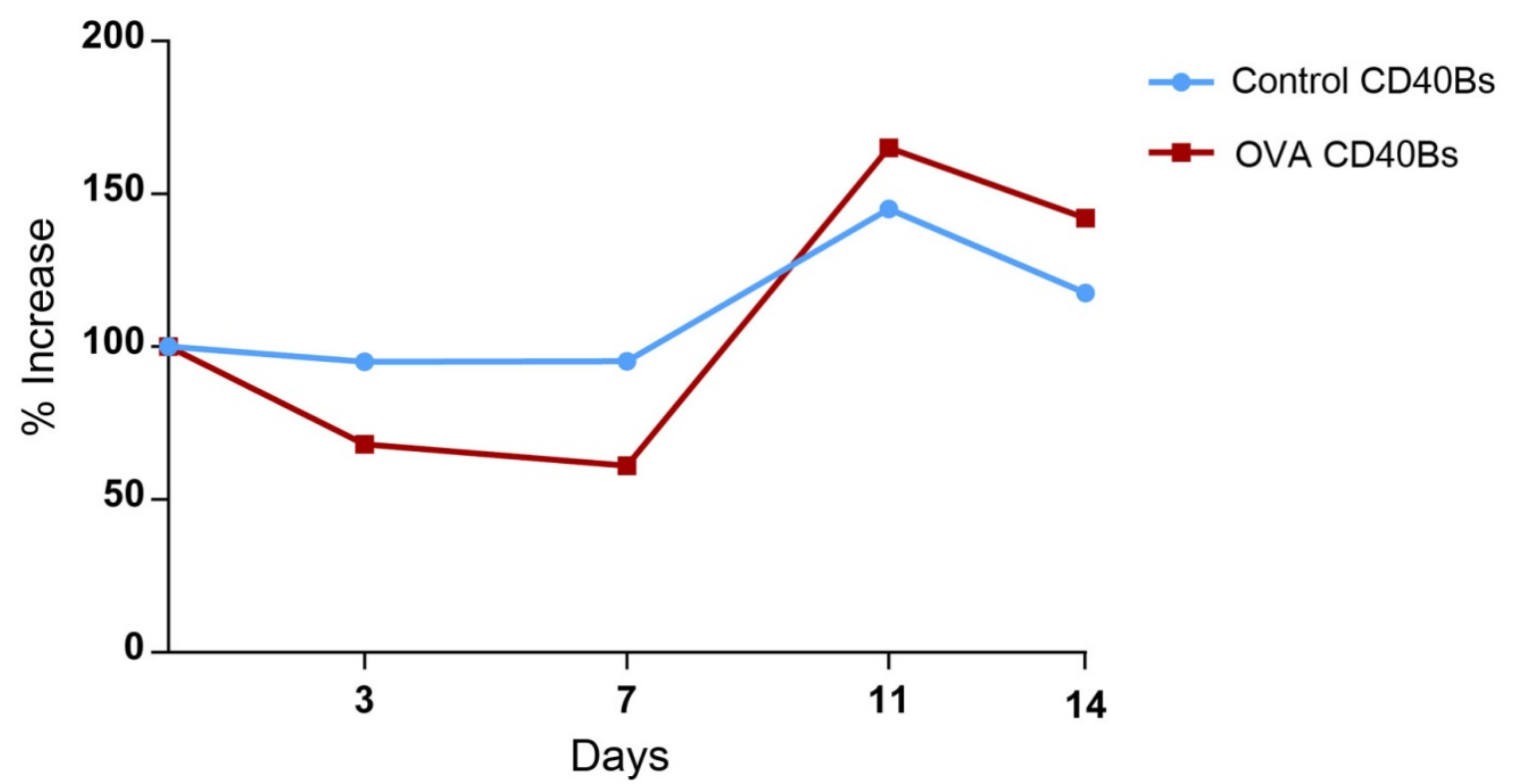

Fig. 3.28 Absolute increase of murine cells in the CD40L culture over 14 days. Purified B cells of immunized or control mice were cultivated on CD40L-expressing HeLa cells over a period of 14 days. The number of viable cells was determined every 3-4 days by trypan blue exclusion test. The absolute increase was determined by calculating the increase between two passages in percentage. One representative growth curve of 5 independent experiments is shown.

Control cultures formed evenly shaped round clusters on day 7 , which stayed stable in size and morphology until day 14 (Fig. 3.29 a-c). Pappenheim staining of control cultures showed a typical lymphocyte appearance with a dark nucleus and a granulated cytoplasm (Fig. 3.30 a-d). As expected, the cell size increased from day 0 to day 7 and the cell shape appeared impaired on day 14.

OVA cultures also formed clusters on day 7. However, their shape was not as round and even as in control cultures and the magnetic beads from the purification process were still visible (Fig. 3.29 d-f). Nevertheless, on day 14 OVA cultures showed a similar morphology to control cultures with large round clusters. In Pappenheim staining, cells of OVA cultures 
showed a dark nucleus and a granulated cytoplasm (Fig. 3.30, e-h). However, compared to control cultures their shape was not as even due to attachment of microbeads. On day 7,11 and 14 cells appeared similar to control cultures with an increase in size and an impaired cell shape on day 14.

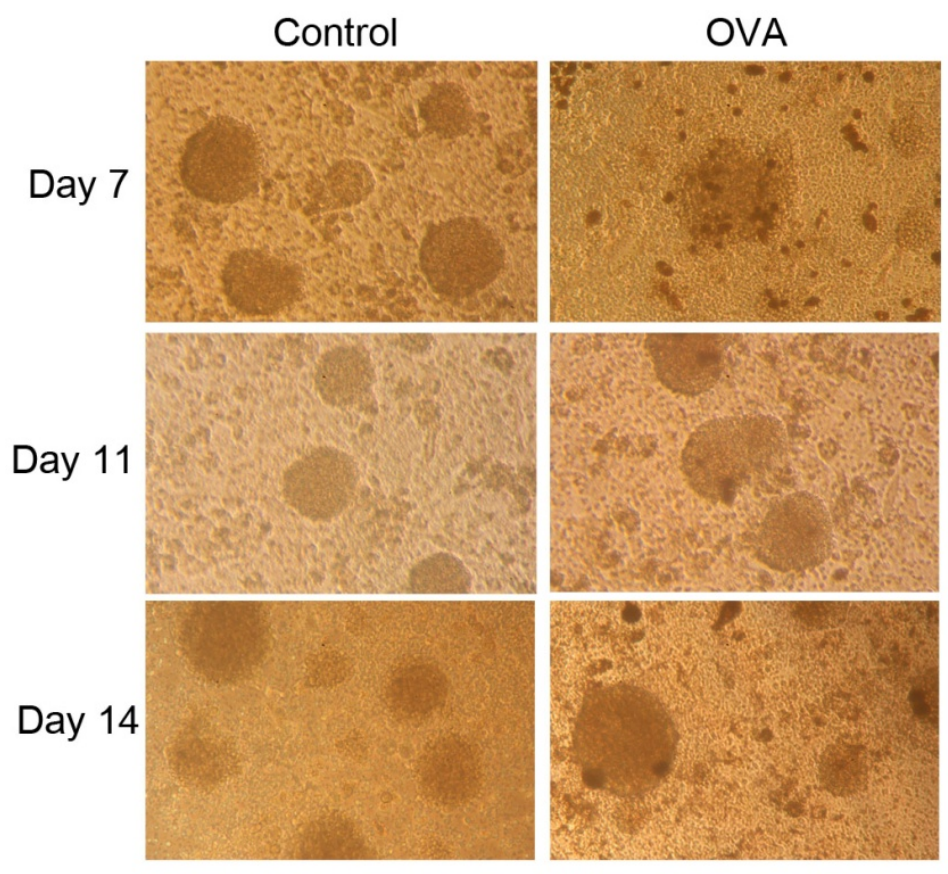

Fig. 3.29 Cluster formation of B cells in CD40L cultures. Purified $B$ cells of immunized or control mice were cultivated on CD40Lexpressing HeLa cells over a period of 14 days. Pictures were taken on day 7,11 and 14 at a 10x magnification using a light optical microscope fitted with a digital camera. Representative pictures of 5 independent experiments are shown.

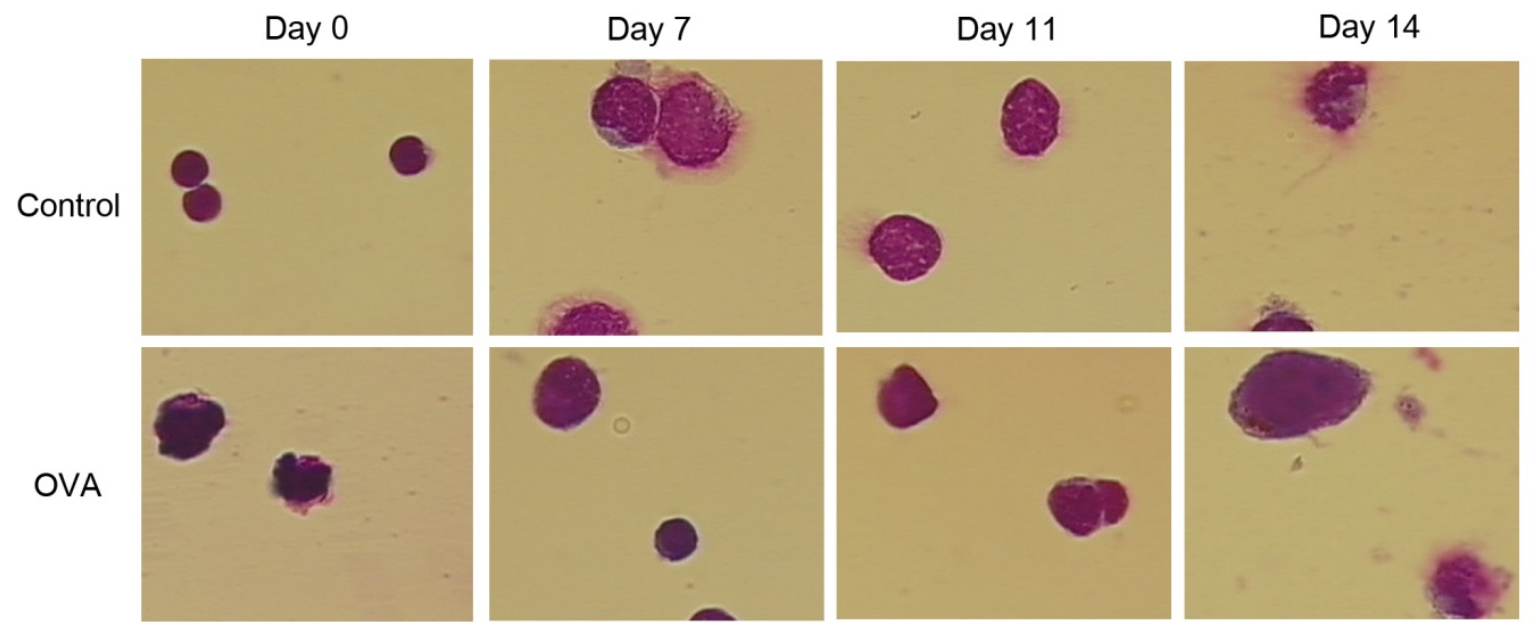

Fig. 3.30 Morphology of B cells in CD40L cultures. Purified B cells of immunized or control mice were cultivated on CD40L-expressing HeLa cells over a period of 14 days. A Pappenheim staining was performed on day $0,7,11$ and 14 of culture and pictures were taken at a $63 x$ magnification using a Zeiss AxioPhot microscope. Representative pictures of 5 independent experiments are shown.

Control and OVA cultures were analyzed by FACS for the percentage of $\mathrm{CD}_{19}{ }^{+} \mathrm{B} 22 \mathrm{O}^{+} \mathrm{B}$ cells and the percentage of OVA-specific among the $\mathrm{CD} 19^{+} \mathrm{B} 22 \mathrm{O}^{+} \mathrm{B}$ cell population. Control 
cultures were $>90 \%$ pure $\mathrm{CD} 19^{+} \mathrm{B} 220^{+} \mathrm{B}$ cells on day 0 and purity stayed stable until day 14

(Fig. 3.31). OVA cultures were about $80 \%$ pure $\mathrm{CD} 19^{+} \mathrm{B} 220^{+} \mathrm{B}$ cells and increased to about $95 \%$ until day 14 . However, the percentage of OVA-specific cells among CD19 ${ }^{+} \mathrm{B} 220^{+} \mathrm{B}$ cells decreased rapidly from more than $60 \%$ on day 0 to about $10 \%$ on day 7 and further decreased until day 14 (Fig. 3.32).

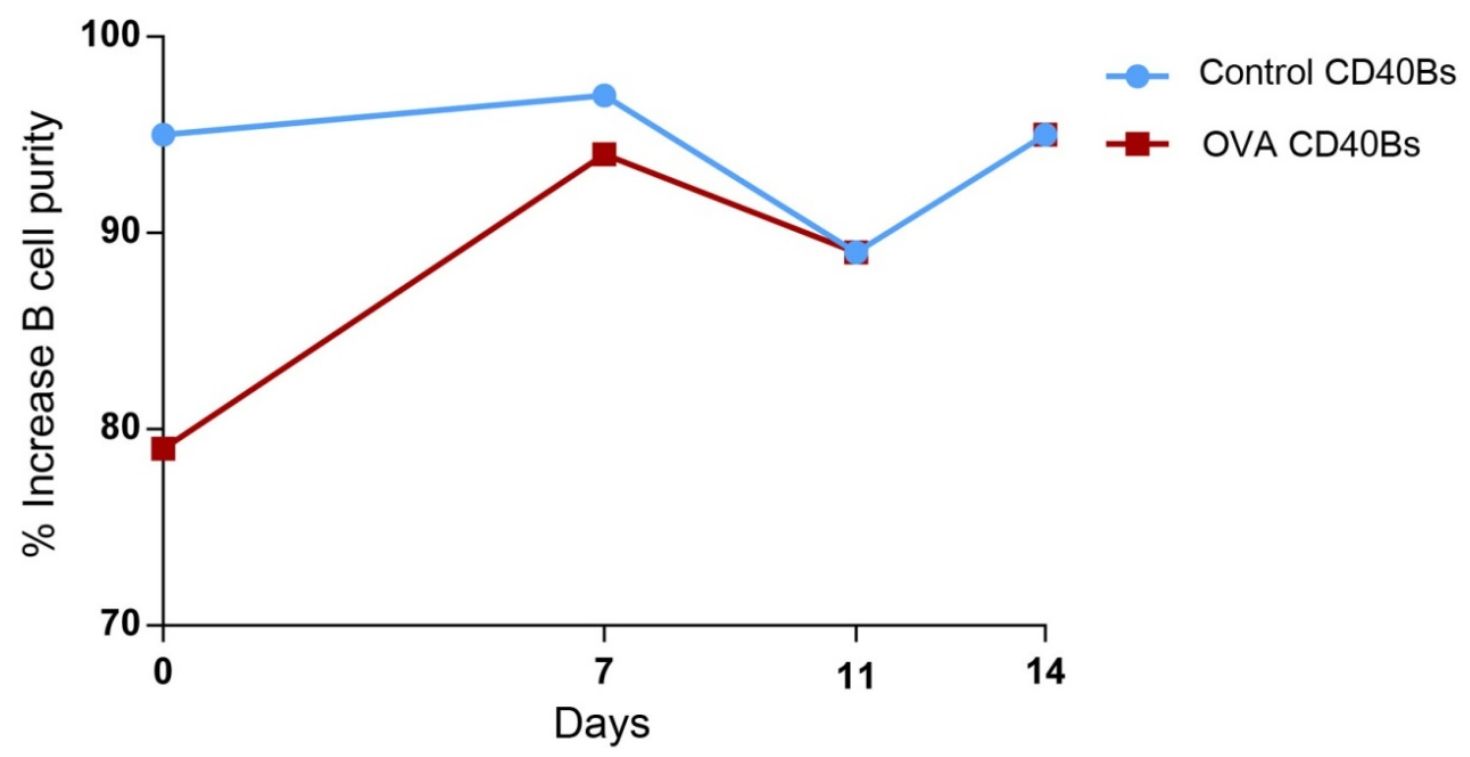

Fig. 3.31 Increase of $\mathrm{CD}^{+} 9^{+} \mathrm{B}^{220^{+}} \mathrm{B}$ cells in CD40L cultures. Purified B cells of immunized or control mice were cultivated on CD40L-expressing HeLa cells over a period of 14 days. Staining for CD19 ${ }^{+}$ B220 ${ }^{+}$B cells was performed on day $0,7,11$ and 14 and the percentage of B cells was determined by flow cytometry analyses. Representative curves out of at least 10 independent experiments are shown.

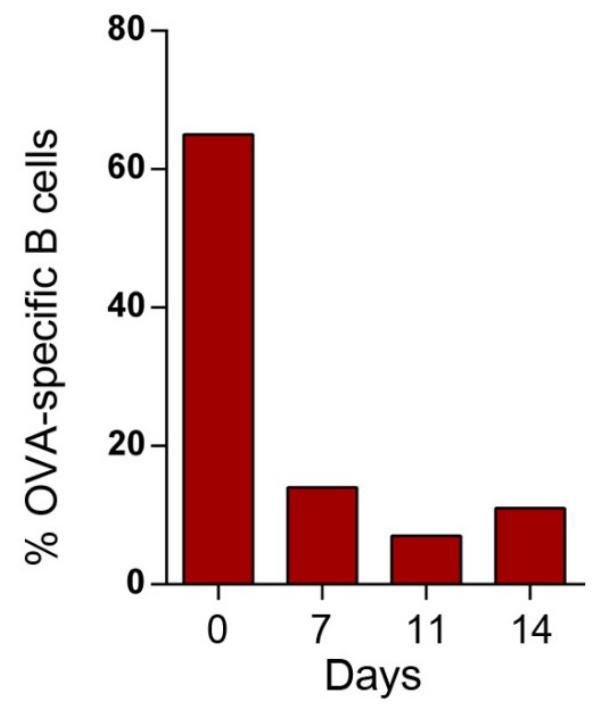

Fig. 3.32 Decrease of OVA-specific $B$ cells in percentage among the $\mathrm{CD}^{2} 9^{+} \mathrm{B220}^{+} \mathrm{B}$ cell population in CD40L cultures. Purified $B$ cells of immunized mice were cultivated on CD40Lexpressing HeLa cells over a period of 14 days. Staining for OVA-specific $\mathrm{CD} 19^{+} \mathrm{B} 22 \mathrm{O}^{+} \mathrm{B}$ cells was performed on day $0,7,11$ and 14 and the percentage of OVA-specific B cells was determined by flow cytometry analyses. Representative bar charts of 3 independent experiments are shown.

Since biotinylated OVA-antigen is used for the enrichment of OVA-specific B cells, the question arose whether the observed decrease in the percentage of OVA-specific B cells is 
simply a failure of the staining method due to a blocked BCR. Therefore, mixed-lymphocyte reactions were performed, in which OVA-specific CD4OB cells from day 1, 7, 11 and 14 were cocultured with OVA-specific OT-I T cells for 5 days. Proliferation of T cells was determined by analyzing the decrease in CFSE labeling. B-to-T-cell ratios were kept constant for the different time points. Therefore, induction of T cell proliferation by $\quad$ CD40B cells should stay stable in case the percentage of OVA-specific B cells in culture was stable, but could simply not be detected. Polyclonal CD40B cells were used as control. As expected, proliferation of $\mathrm{T}$ cells decreased with decreasing B-to-T-cell ratios (Fig. 3.33). Moreover, induction of proliferation decreased with increasing time in CD40B culture (day 7-14) thereby confirming the decreasing percentages of OVA-specific B cells as determined by FACS (Fig. 3.32).

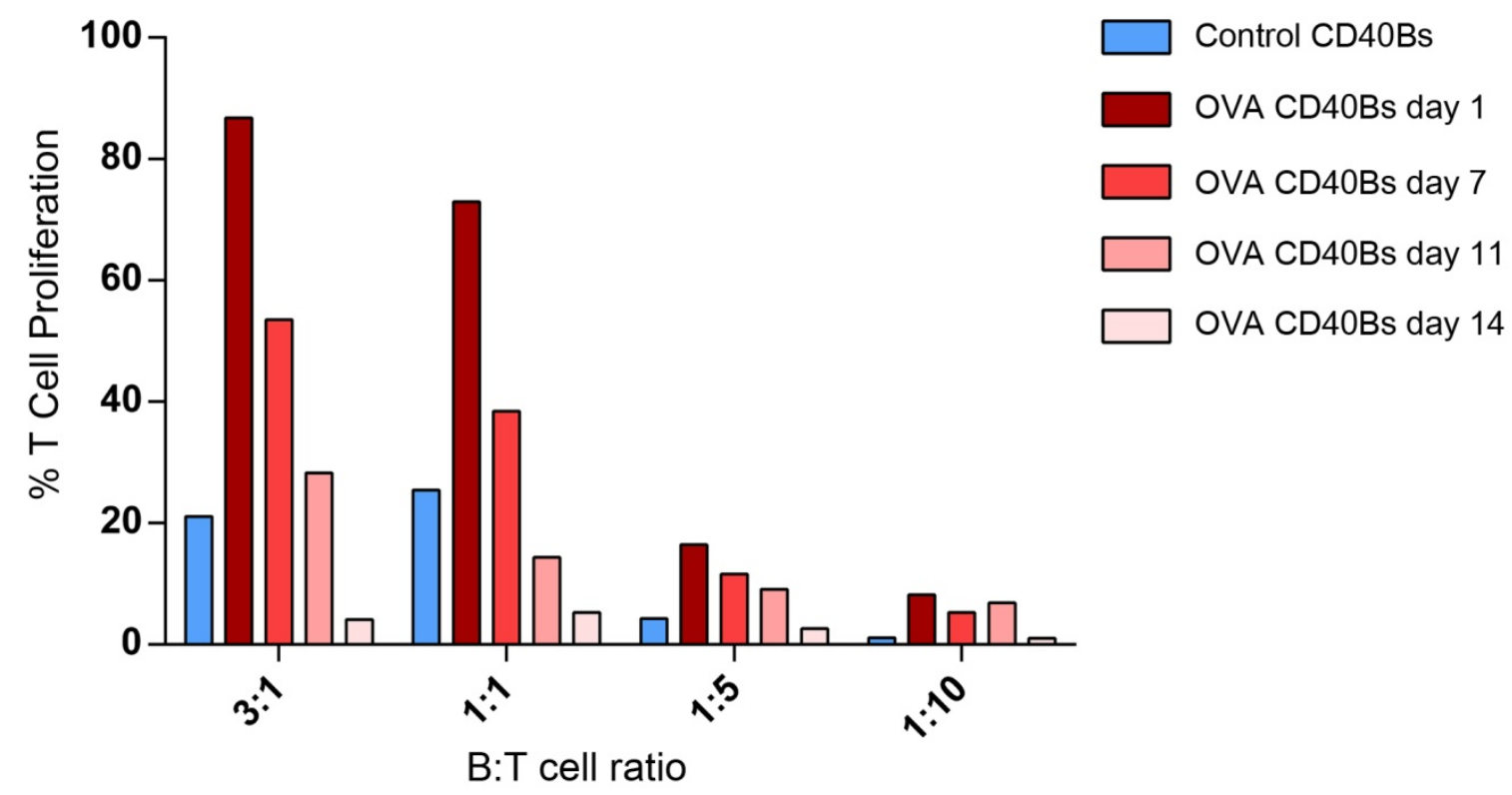

Fig. 3.33 T cell proliferation induced by CD40-activated B cells. Purified B cells of immunized mice were cultivated on CD40L-expressing HeLa cells over a period of 14 days. Cells from day 1, 7, 11 and 14 were cocultured in different ratios together with OT-I T cells for 5 days. Proliferation of T cells was determined by flow cytometry analyses by a decrease in CFSE-staining. CD4OB cells of nonimmunized mice served as control. Bar charts represent one independent experiment.

Another possible explanation for the decrease in the percentages of OVA-specific B cells would be their differentiation into plasma cells and the subsequent loss of the BCR. Therefore, OVA cultures were analyzed for their expression of the plasma cell marker

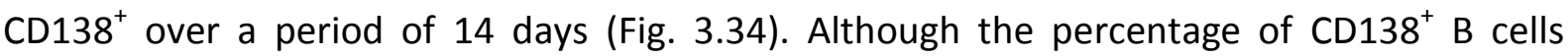
increased in OVA cultures on day 7, it was not high enough to account for the decrease in the percentage of OVA-specific B cells. Furthermore, in Pappenheim staining of OVA cultures 
(Fig. 3.29) no plasma cells could be detected, which would appear larger in size and with a more distinct cytoplasm.

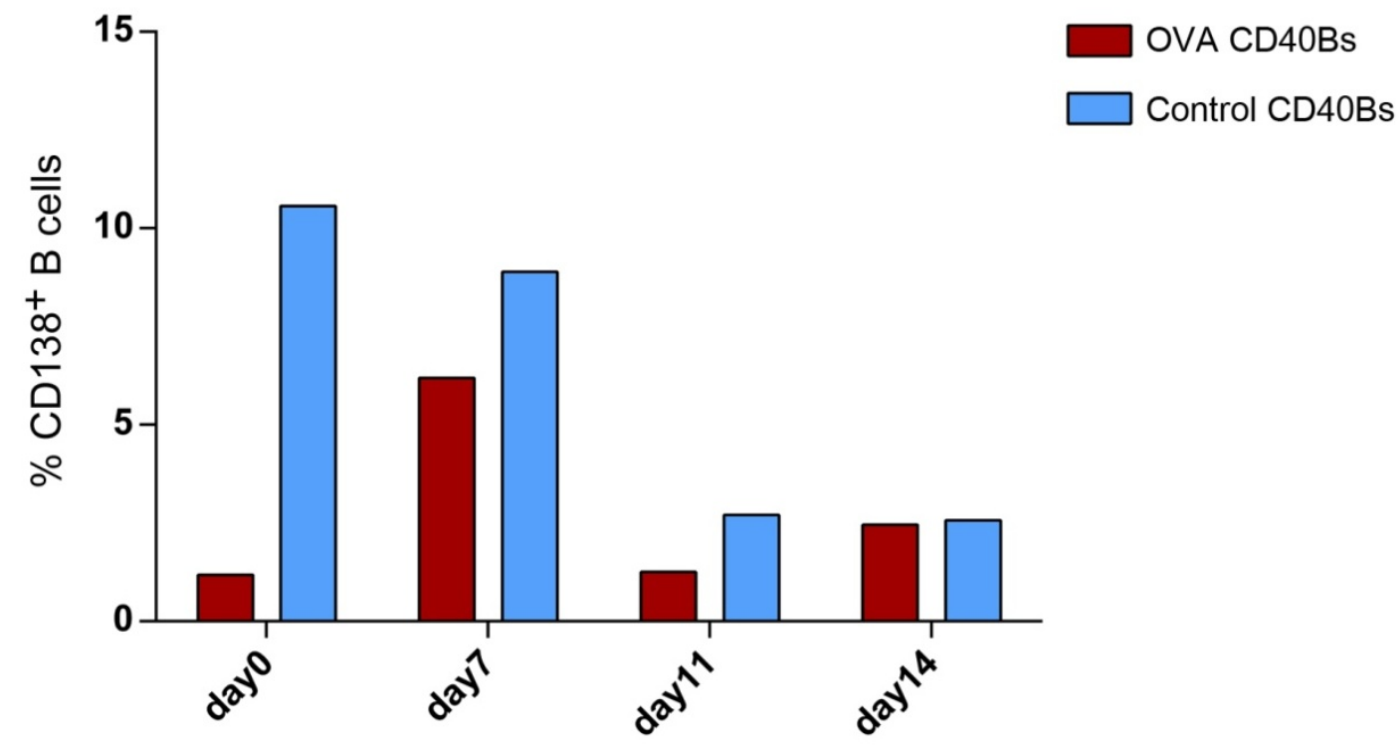

Fig. 3.34 Percentage of CD138 ${ }^{+}$B cells in CD40L cultures. Purified B cells of immunized or control mice were cultivated on CD40L-expressing HeLa cells over a period of 14 days. Staining for CD138 was performed on day $0,7,11$ and 14 and the percentage of $\mathrm{CD} 138^{+} \mathrm{B}$ cells was determined by flow cytometry analyses. Representative bar charts of 3 independent experiments are shown.

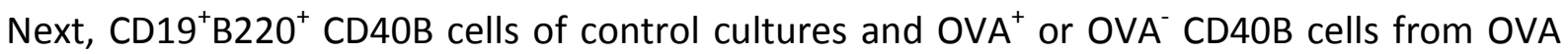
cultures were stained for Annexin $V$ as an indicator for cell apoptosis (Koopman et al. 1994). $\mathrm{OVA}^{+} \mathrm{CD} 40 \mathrm{~B}$ cells of OVA cultures showed a slight increase in Annexin $\mathrm{V}^{+}$staining (Fig. 3.35, left column) compared to control CD4OB cells with or OVA ${ }^{-} \mathrm{CD} 40 \mathrm{~B}$ cells (Fig. 3.35, right and middle column, respectively). However, this difference was not high enough to explain the decrease in the percentage of OVA-specific B cells. 


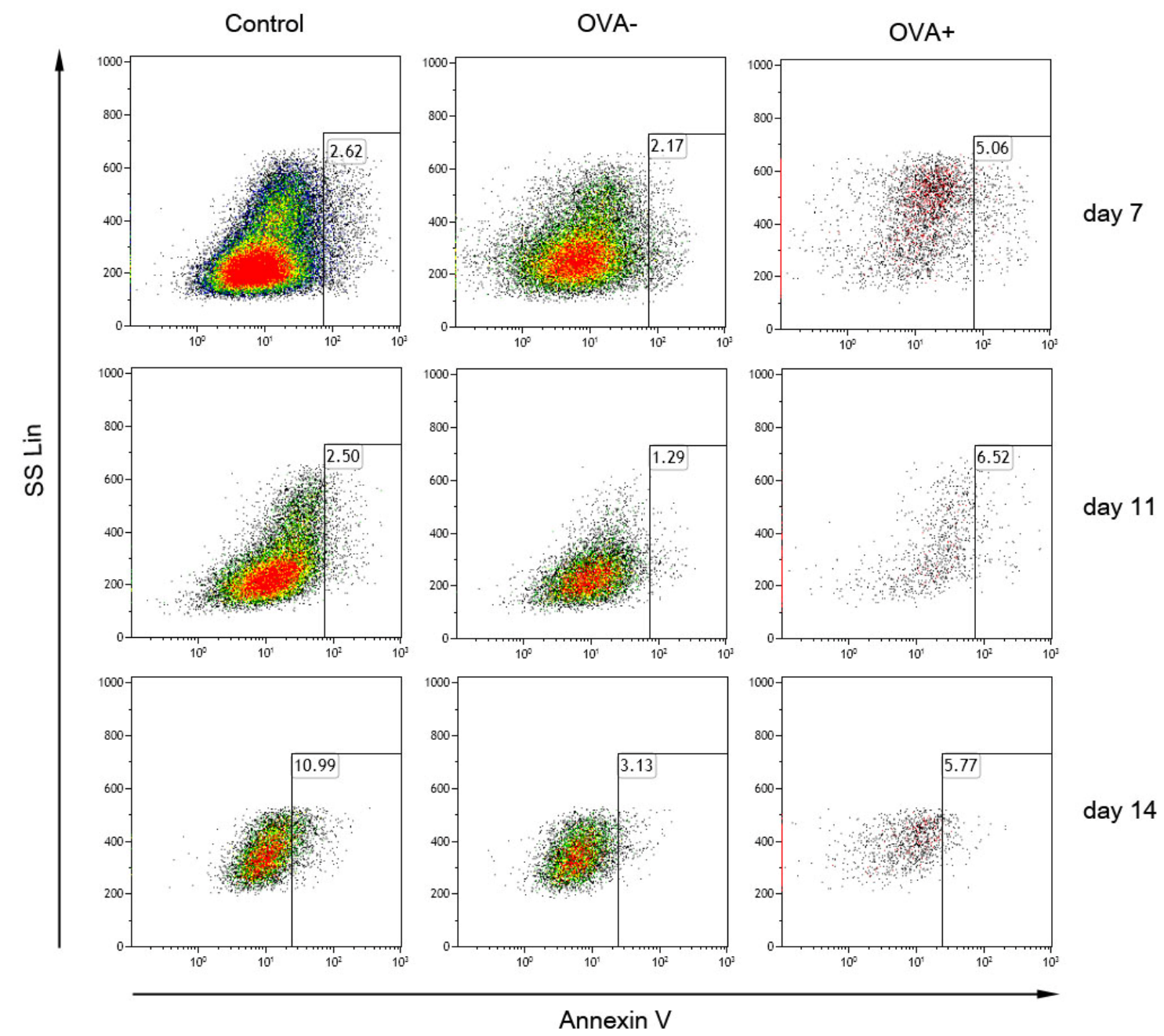

Fig. 3.35 Annexin V staining of CD19 ${ }^{+} \mathrm{B220}^{+} \mathrm{B}$ cells in CD40L cultures. Purified B cells of immunized or control mice were cultivated on CD40L-expressing HeLa cells over a period of 14 days. Staining for Annexin $\mathrm{V}$ was performed on day $0,7,11$ and 14 and the percentage of Annexin $\mathrm{V}^{+}$OVA+ and OVA- B cells of immunized mice or control $B$ cells of non-immunized mice was determined by flow cytometry analyses. Representative plots of 3 independent experiments are shown. Numbers indicate the percentage of Annexin $\mathrm{V}^{+} \mathrm{B}$ cells.

Having ruled out the explanations of an inaccurate staining, the differentiation into plasma cells or an increased apoptosis, OVA-specific B cells were stained with CFSE in order to analyze their proliferation behavior. CFSE segregates equally between daughter cells upon cell division, thereby resulting in the sequential halving of cellular fluorescence intensity with each successive generation. Interestingly, FACS analyses revealed that OVA-specific B cells proliferated less (Fig. 3.36, black line) than B cells in control cultures (Fig. 3.36, filled line) or OVA $^{-}$B cells in OVA cultures (Fig. 3.36, grey line). Detailed analysis of the cultures by Flow Jo's proliferation tool (Flow Jo Version 10, Tree Star) revealed a much lower percentage of dividing cells among OVA-specific B cells compared to control or OVA` B cells over the whole culture period of 14 days (Table 3.1). Accordingly, the division index, which reflects the 
average number of cell divisions that a cell in the original population has undergone, was between 20 and 30 fold smaller on day 7 and day 11 in OVA-specific B cells than in control or OVA $^{-}$B cells, respectively (Table 3.1). Although there was no difference between the three populations in the proliferation index (Table 3.1), which only takes into account the divisions of cells that actually respond, these data indicate a clear proliferative disadvantage of OVAspecific B cells compared to OVA'B cells from the same cultures. The OVA-specific B cells seem to stay in culture, but since they proliferate less than OVA- B cells their percentage in the overall B cell population decreases constantly over a period of 14 days. Therefore, all following experiments were performed with OVA-specific B cells that were cultivated in the CD40 system for a maximum of 2 days.

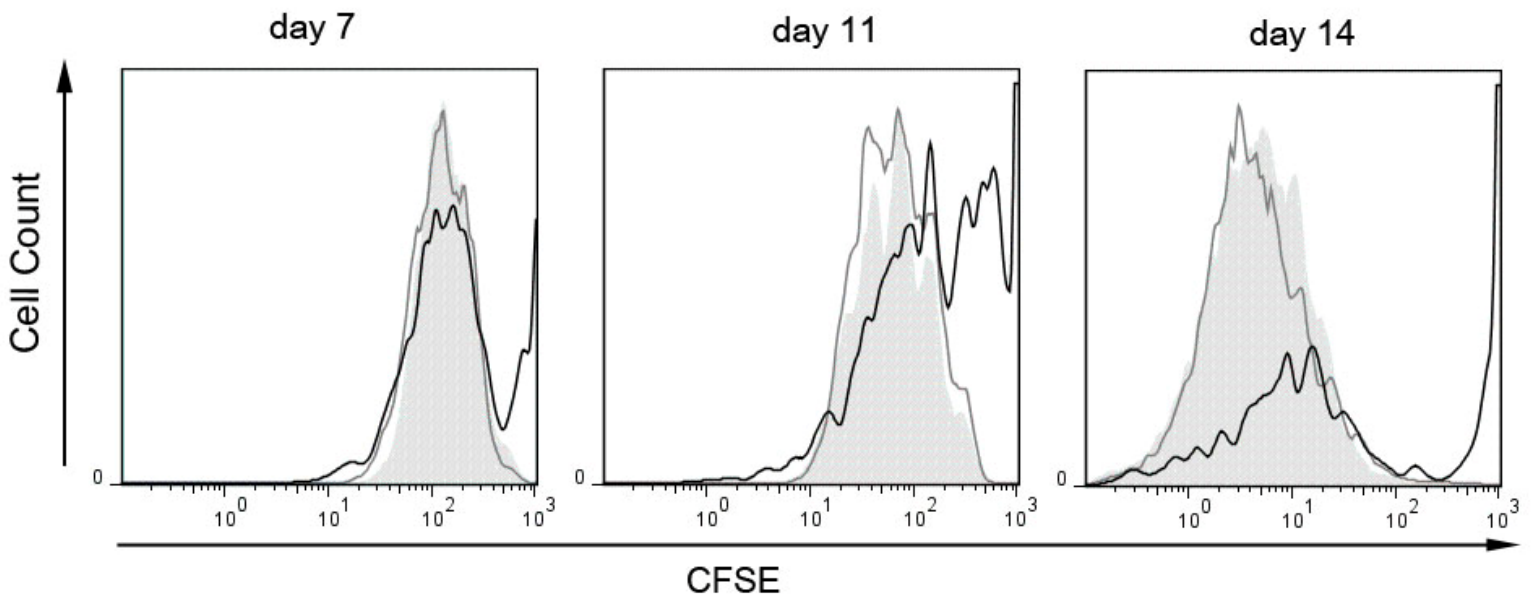

Fig. 3.36 CFSE staining of B cells in CD40L cultures. Purified B cells of immunized or control mice were stained with CFSE and cultivated on CD40L-expressing HeLa cells over a period of 14 days. Proliferation of OVA+ (black line) and OVA- B cells (grey line) of immunized mice and control B cells (filled) of non-immunized mice was determined on day 7,11 and 14 measuring the decrease in CFSE by flow cytometry analyses. Histograms represent one independent experiment. 
Table 3.1 Proliferation Analysis of B cells in CD40L culture. Purified B cells of immunized or control mice were stained with CFSE and cultivated on CD4OL-expressing HeLa cells over a period of 14 days. Proliferation of OVA-specific and OVA-negative CD4OB cells of immunized mice and control CD4OB cells of non-immunized mice was determined on day 7, 11 and 14 and analyzed with the Flow Jo Proliferation Tool (Flow Jo Version 10).

\begin{tabular}{|l|r|r|r|}
\hline & \% Divided Cells & \multicolumn{1}{|l|}{ Division Index } & \multicolumn{1}{|l|}{ Proliferation Index } \\
\hline Day 7 & & & \\
\hline Control & 56.6 & 1 & 1.77 \\
\hline OVA- & 87.4 & 1.58 & 1.81 \\
\hline OVA+ & 3.68 & 0.0534 & 1.45 \\
\hline & & & \\
\hline Day 11 & & & 1.71 \\
\hline Control & 69.4 & 1.19 & 2.06 \\
\hline OVA- & 71.4 & 1.48 & 2.1 \\
\hline OVA+ & 3.74 & 0.0784 & 1.08 \\
\hline & & & 1.24 \\
\hline Day 14 & & & 1.44 \\
\hline Control & 3.69 & 0.04 & \\
\hline OVA- & 18.2 & 0.226 & \\
\hline OVA+ & 2.9 & 0.0417 & \\
\hline
\end{tabular}

\subsubsection{Murine Antigen-Specific B Cells Upregulate Activation Markers upon CD40L}

\section{Stimulation}

OVA-specific and polyclonal B cells that were kept in the CD40 culture for 1 day were analyzed for the expression levels of several surface markers in order to confirm their antigen-presenting phenotype. Bar charts represent results of at least three independent experiments. Expression levels on day 1 of culture of the different CD4OB cells populations (OVA-specific and OVA-negative from immunized mice and control CD40B cells from nonimmunized mice) were normalized to the expression levels of the respective population on day 0.

There was no difference in expression of the B cell marker B220 in any of the observed CD40B cell populations on day 1 compared to their respective population on day 0 (Fig. 3.37). The B cell marker CD19 was upregulated in all CD40B cell populations from day 0 to day 1 (Fig. 3.38), although the difference was only significant in control cells $(121.2 \% \pm$ 47.59). 

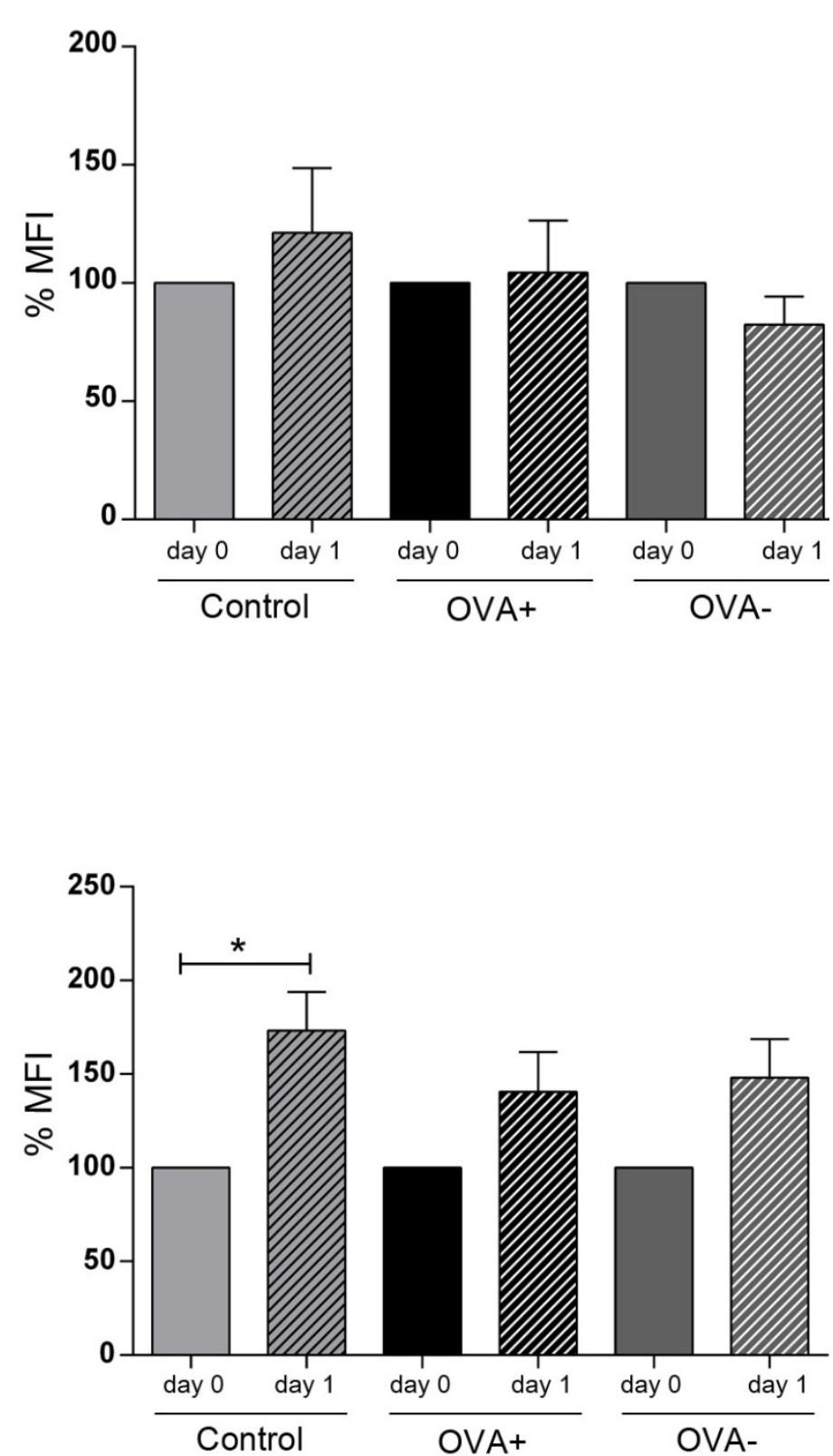

Fig. 3.37 B220 expression in OVAspecific CD40B cells on day 1 of culture. The mean fluorescent intensity (MFI) of B220 of control CD40B cells, OVA-specific (OVA+) or OVA-negative (OVA-) CD4OB cells on day 1 of the CD40 culture was determined by flow cytometry and values were normalized to the expression levels in B cells of the respective populations on day 0 . Bar charts show mean values \pm SEM of three independent experiments. Significant differences calculated with ordinary one-way ANOVA were not detected.

Fig. 3.38 CD19 expression in OVAspecific CD40B cells on day 1 of culture. The mean fluorescent intensity (MFI) of CD19 of control CD40B cells, OVA-specific (OVA+) or OVA-negative (OVA-) CD40B cells on day 1 of the CD40 culture was determined by flow cytometry and values were normalized to the expression levels in B cells of the respective populations on day 0 . Bar charts show mean values \pm SEM of three independent experiments. Significant differences were calculated with ordinary one-way ANOVA are marked by an asterisk. $* p \leq 0.05$.

As expected, the percentage of $\operatorname{IgG} 1^{+} \mathrm{B}$ cells in the three $\mathrm{CD} 40 \mathrm{~B}$ cell populations increased from day 0 to day 1 in culture. The increase was not significant for control CD4OB cells (12.74 $\% \pm 1.01)$, OVA-negative CD40B cells $(74.20 \% \pm 0.81)$ or OVA-specific CD4OB cells $(90.52 \% \pm$ 1.23) on day 1 (Fig. 3.39). 


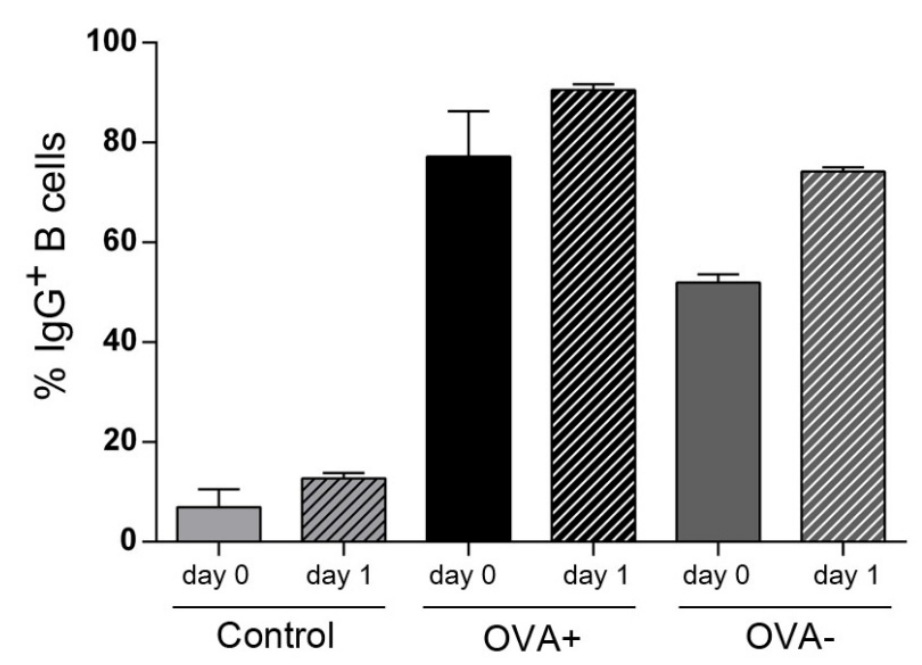

Fig. 3.39 Percentage of $\operatorname{IgG1}^{+}$ CD40B cells on day 1 of culture. The percentage of $\operatorname{IgG1}^{+} \mathrm{B}$ cells among control CD40B cells, OVAspecific (OVA+) or OVA-negative (OVA-) CD40B cells on day 1 of the CD40 culture was determined by flow cytometry and compared to the percentages of the respective populations on day 0 . Bar charts show mean values \pm SEM of three independent experiments.

Significant differences were calculated with ordinary one-way ANOVA are marked by an asterisk. * $p \leq 0.05$.

Moreover, a significant difference in $\operatorname{IgD}^{+} \mathrm{CD} 40 \mathrm{~B}$ cells was observed in control CD40B cells (55.33\% $\% 4.58)$ and OVA-negative CD40B cells $(21.10 \% \pm 7.82)$ on day 1 (Fig. 3.40 ), but the difference was not significant in OVA-specific CD4OB cells $(22.41 \% \pm 8.41)$. Interestingly, $\operatorname{IgM}^{+}$B cells were increased on day 1 in control CD40B cells (96.32 \% \pm 0.98$)$, OVA-specific CD40B cells $(37.83 \% \pm 2.14)$ and OVA-negative CD40B cells $(82.33 \% \pm 5.22$; Fig. 3.41). However, the differences were not significant. These data indicate that B cells further undergo class-switch in the CD40 culture and on day 1 seems to be still in a transitional state from $\operatorname{IgD}^{-} \operatorname{lgM}^{\text {high }} \operatorname{lgG}^{+}$to $\operatorname{lgD}^{-} \operatorname{lgM}^{\text {low }} \operatorname{lgG}^{\text {high }} B$ cells.

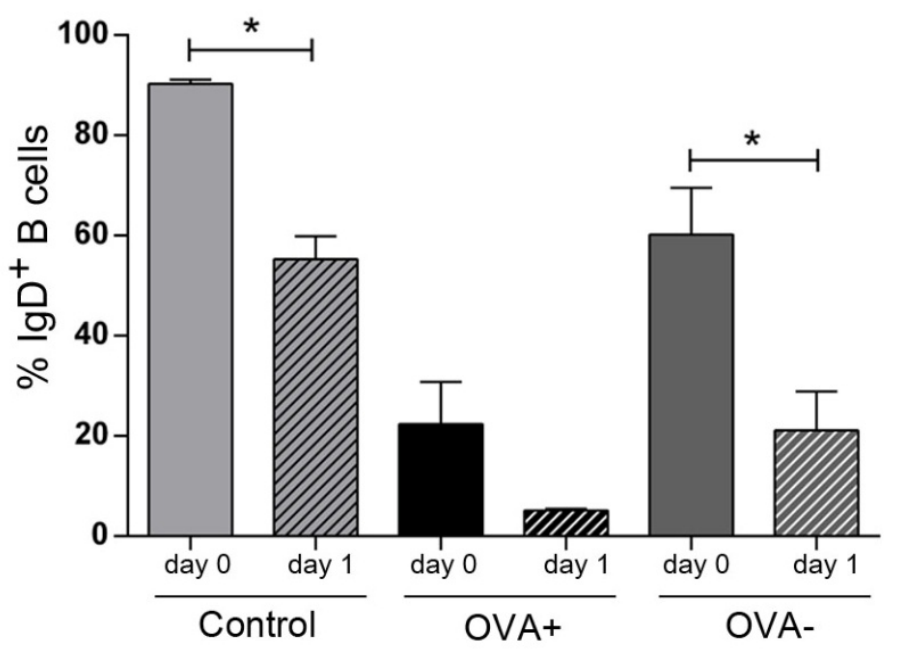

Fig. 3.40 Percentage of $\operatorname{IgD}^{+} \mathrm{CD} 40 \mathrm{~B}$ cells on day 1 of culture. The percentage of $\operatorname{lgD}^{+} B$ cells among control CD40B cells, OVA-specific (OVA+) or OVA-negative (OVA-) CD40B cells on day 1 of the CD40 culture was determined by flow cytometry and compared to the percentages of the respective populations on day 0 . Bar charts show mean values \pm SEM of three independent experiments. Significant differences were calculated with ordinary one-way ANOVA are marked by an asterisk. * $p \leq 0.05$. 


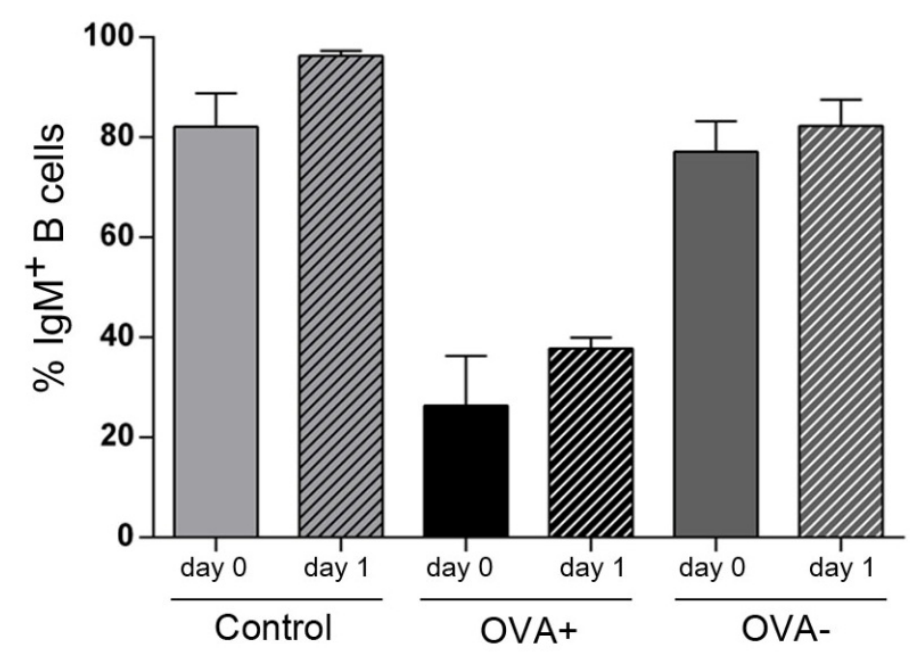

Fig. 3.41 Percentage of $\operatorname{IgM}^{+}$CD40B cells on day 1 of culture. The percentage of $\operatorname{lgM}^{+} B$ cells among control CD40B cells, OVA-specific (OVA+) or OVA-negative (OVA-) CD40B cells on day 1 of the CD40 culture was determined by flow cytometry and compared to the percentages of the respective populations on day 0 . Bar charts show mean values \pm SEM of three independent experiments. Significant differences calculated with ordinary one-way ANOVA were not detected.

The expression of MHC I molecules was highly upregulated on day 1 (Fig. 3.42) in control CD40B cells $(1026.0 \% \pm 48.91)$, OVA-specific CD40B cells $(507.1 \% \pm 10.81)$ and OVAnegative CD40B cells $(901.1 \% \pm 40.38)$. The same was observed for MHC class II molecules (Fig. 3.43) for control CD4OB cells (714.1 \% \pm 155.1), OVA-specific CD4OB cells $(342.9 \% \pm$ 11.42 ) and OVA-negative CD4OB cells (757.0\% \pm 188.7$)$.

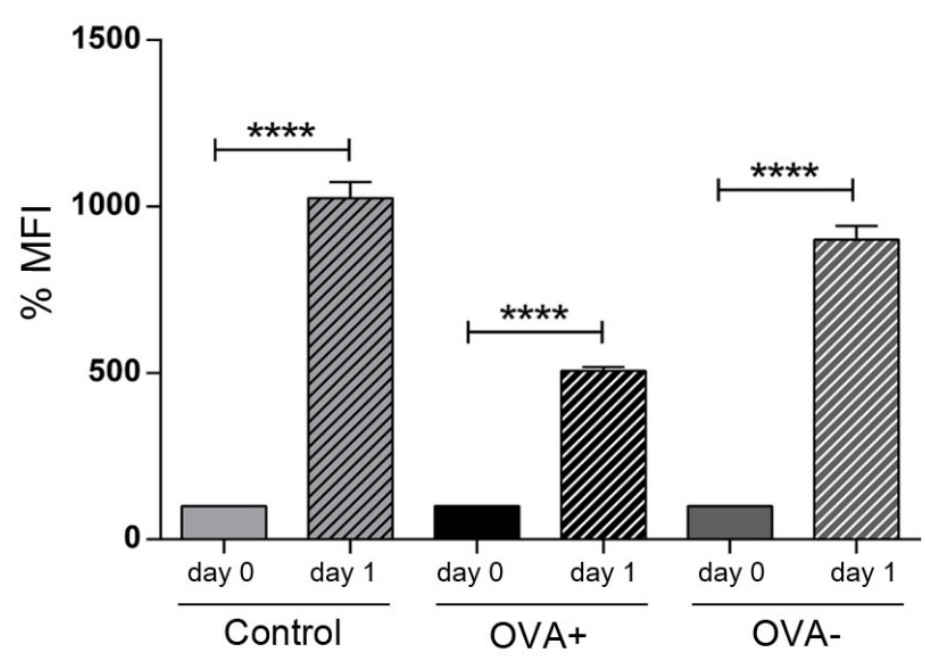

Fig. 3.42 MHC I expression in OVAspecific CD40B cells on day 1 of culture. The mean fluorescent intensity (MFI) of MHC I of control CD40B cells, OVA-specific (OVA+) or OVA-negative (OVA-) CD4OB cells on day 1 of the CD40 culture was determined by flow cytometry and values were normalized to the expression levels in B cells of the respective populations on day 0 . Bar charts show mean values \pm SEM of three independent experiments. Significant differences were calculated with ordinary one-way ANOVA are marked by an asterisk. $* * * * \mathrm{p} \leq 0.0001$. 


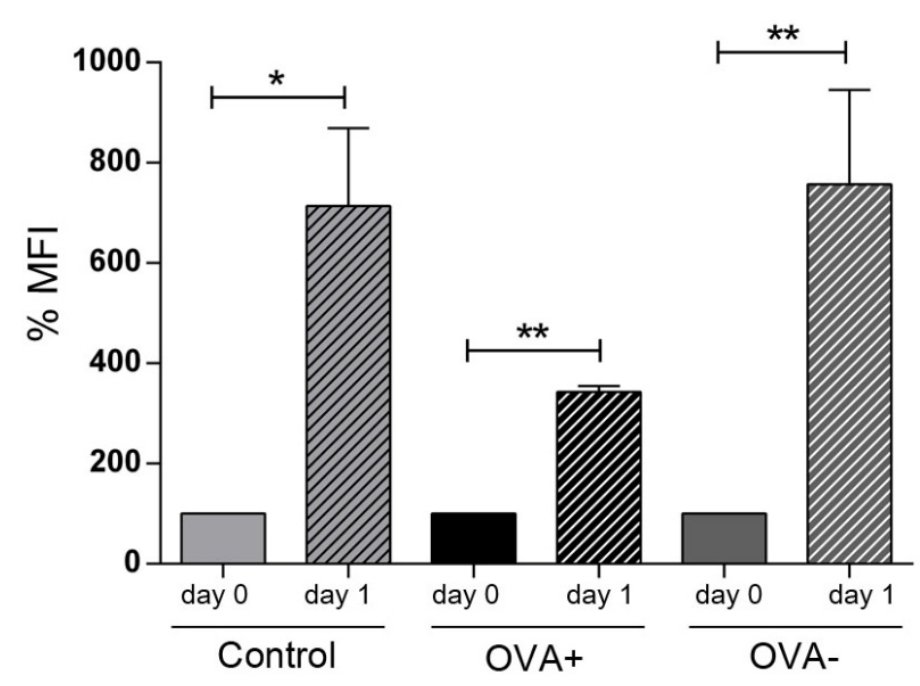

Fig. 3.43 MHC II expression in OVAspecific CD40B cells on day 1 of culture. The mean fluorescent intensity (MFI) of MHC II of control CD40B cells, OVA-specific (OVA+) or OVA-negative (OVA-) CD40B cells on day 1 of the CD40 culture was determined by flow cytometry and values were normalized to the expression levels in B cells of the respective populations on day 0 . Bar charts show mean values \pm SEM of three independent experiments. Significant differences were calculated with ordinary one-way ANOVA are marked by an asterisk. $* p \leq 0.05, * * p \leq 0.01$, $* * * * p \leq 0.0001$.

Moreover, the expression of the costimulatory molecule CD86 (Fig. 3.44) was significantly upregulated in OVA-specific CD4OB cells $(354.3 \% \pm 45.18)$ and OVA-negative CD40B cells (315.6 \% \pm 81.78$)$ and was even higher in control CD4OB cells $(425.8 \% \pm 57.90)$.

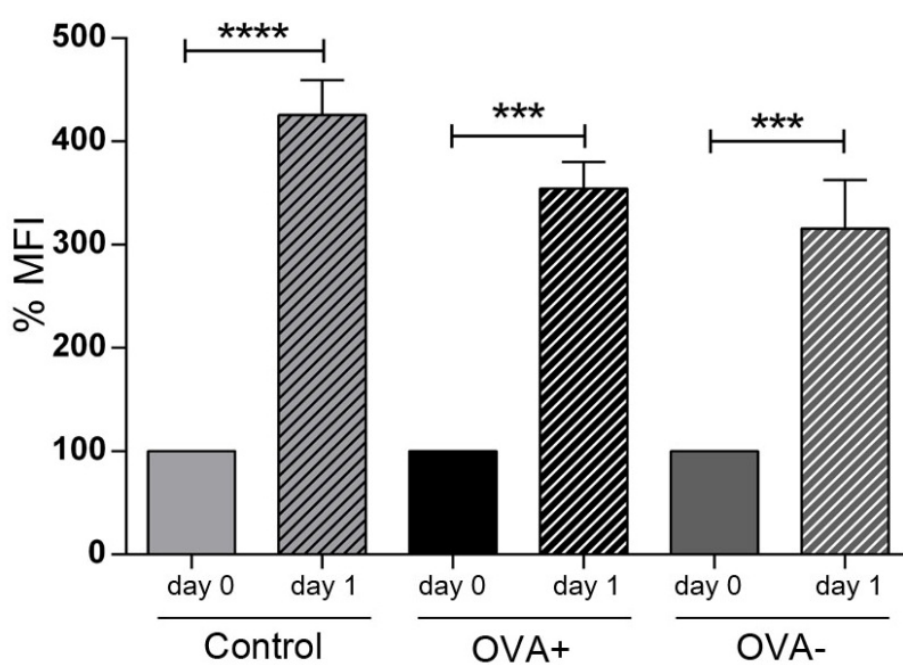

Fig. 3.44 CD86 expression in OVAspecific CD40B cells on day 1 of culture. The mean fluorescent intensity (MFI) of CD86 of control CD40B cells, OVA-specific (OVA+) or OVA-negative (OVA-) CD40B cells on day 1 of the CD40 culture was determined by flow cytometry and values were normalized to the expression levels in B cells of the respective populations on day 0 . Bar charts show mean values \pm SEM of three independent experiments. Significant differences were calculated with ordinary one-way ANOVA are marked by an asterisk. $* * * \mathrm{p} \leq 0.001, * * * * \mathrm{p} \leq 0.0001$.

Although OVA-specific CD40B cells also upregulated the expression of CD80 (137.9\% \pm 1.00 ;

Fig. 3.45) the difference to the expression on day 0 was not significant. No differences in CD80 expression were observed in control CD40B cells $(96.71 \% \pm 24.69)$ or OVA-negative CD40B cells $(76.81 \% \pm 15.77)$ from day 0 to day 1 . 


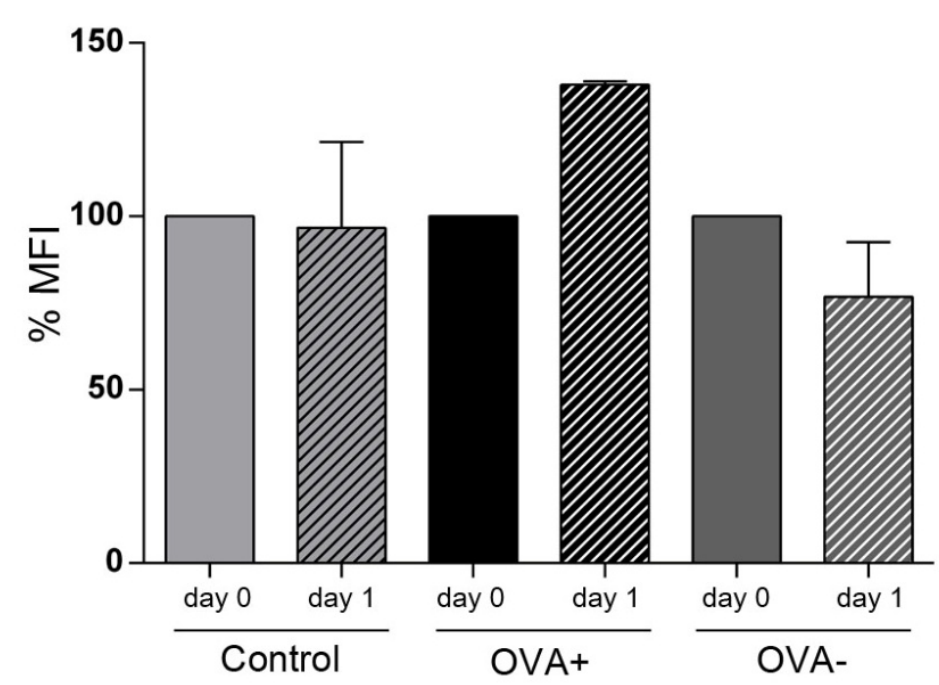

Fig. 3.45 CD80 expression in OVAspecific CD40B cells on day 1 of culture. The mean fluorescent intensity (MFI) of CD80 of control CD40B cells, OVA-specific (OVA+) or OVA-negative (OVA-) CD40B cells on day 1 of the CD40 culture was determined by flow cytometry and values were normalized to the expression levels in B cells of the respective populations on day 0 . Bar charts show mean values \pm SEM of three independent experiments. Significant differences calculated with ordinary one-way ANOVA were not detected.

The percentage of $\mathrm{CD} 138^{+} \mathrm{B}$ cells was increased in all three populations from day 0 to day 1 (control CD40Bs: $4.67 \% \pm 1.28$; OVA-specific CD40Bs: $11.08 \% \pm 3.11$; OVA-negative CD40Bs: $3.41 \% \pm 0.35$ ), although the differences were not significant (Fig. 3.46).

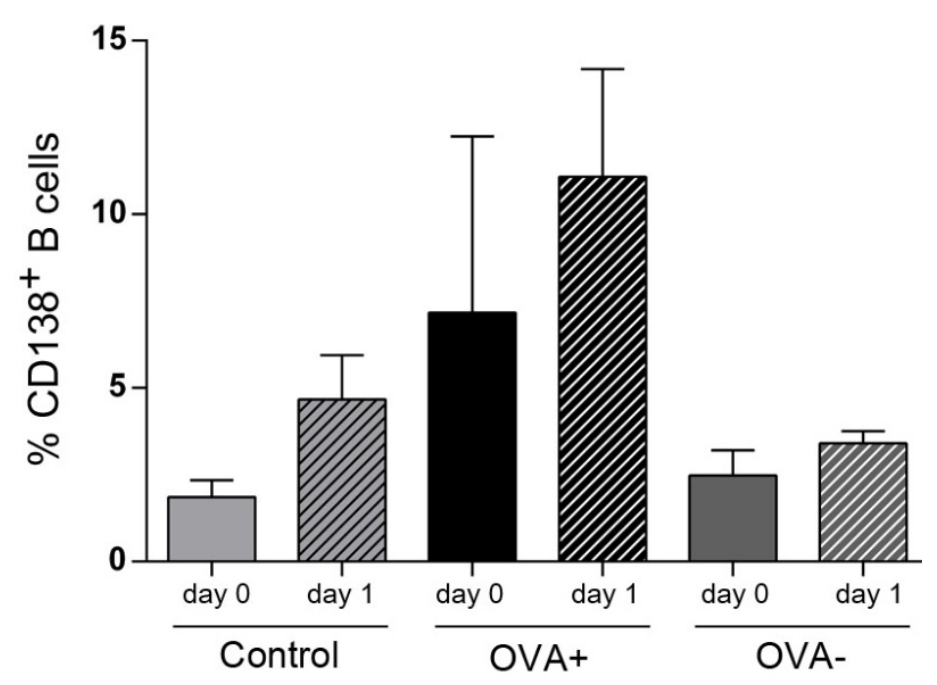

Fig. 3.46 Percentage of $\mathrm{CD} 138^{+}$ CD40B cells on day 1 of culture. The percentage of $\mathrm{CD} 138^{+} \mathrm{B}$ cells among control CD40B cells, OVAspecific (OVA+) or OVA-negative (OVA-) CD40B cells on day 1 of the CD40 culture was determined by flow cytometry and compared to the percentages of the respective populations on day 0 . Bar charts show mean values \pm SEM of three independent experiments. Significant differences calculated with ordinary one-way ANOVA were not detected.

These data clearly indicate that B cells undergo class-switching when stimulated with the CD40 ligand and that antigen-specific B cells upregulate costimulatory and MHC molecules, which has already been shown for polyclonal CD40B cells (Ahmadi et al. 2008, Liebig et al. 2010).

\subsubsection{Human Antigen-Specific B Cells are Activated by Stimulation with the CD40L}

Purified HBV-specific B cells ( $15 \%$ B cell purity, $30 \%$ HBV-specific B cells) were kept in the CD40 culture on CD40L-expressing NIH feeder cells for up to 14 days. B cells were harvested 
on day 7, 11 and 14 and recultivated on fresh feeder cells. The absolute increase of cells in culture was determined (Fig. 3.47) and pictures (Fig. 3.48) were taken on these days. PBMCs isolated from non-vaccinated donors ( $15 \%$ B cells) served as controls.

The number of cells in HBV-cultures increased from day 0 to 7 , while the number of cells in control cultures decreased slightly (Fig. 3.47). From day 7 to day 11 , the control cultures started to proliferate, resulting in a 2 fold increase of the initial culture on day 14 . While proliferation of HBV-specific cultures stayed stable from day 7 to day 11 , they highly proliferated afterwards resulting in a 3.5 fold expansion of initial cultures.

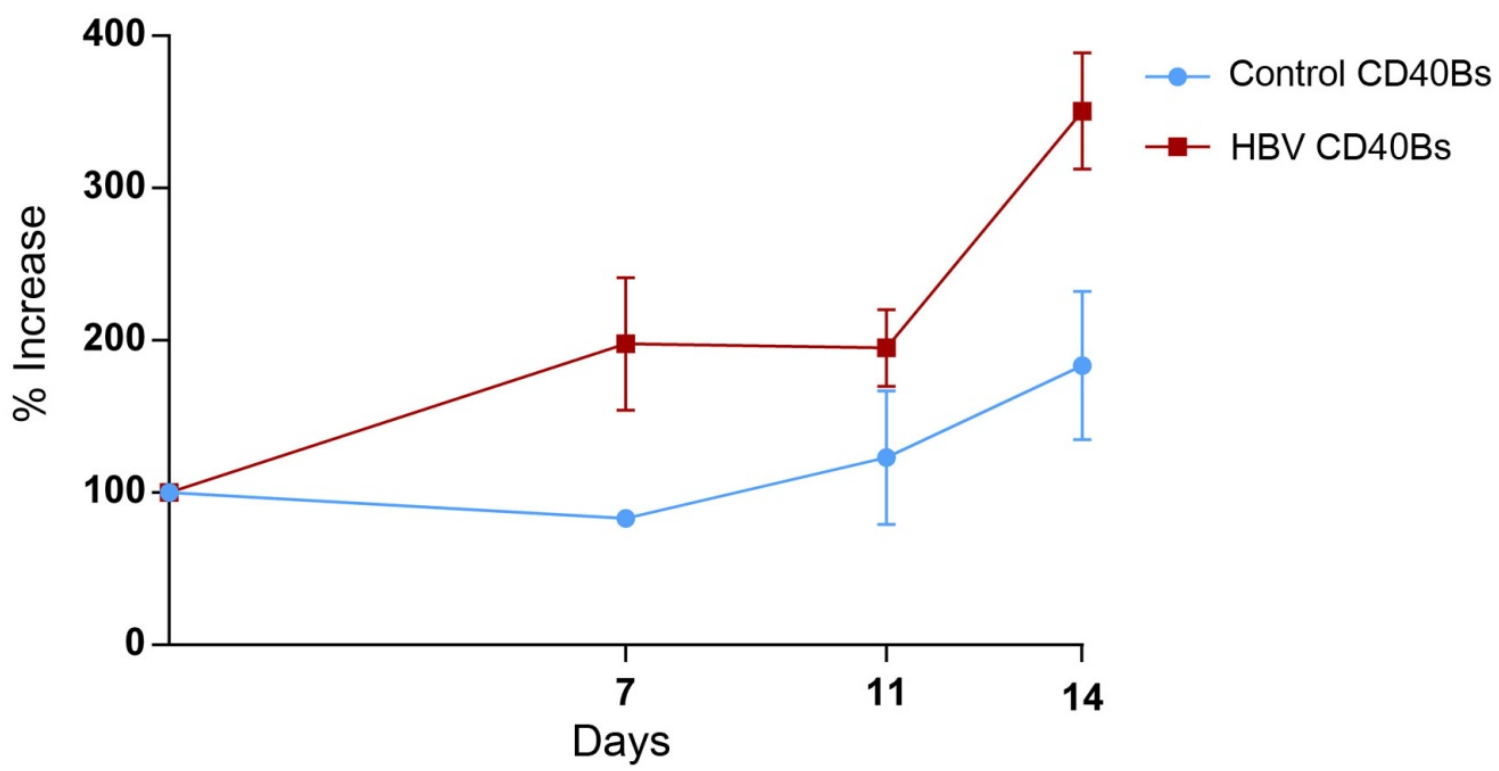

Fig. 3.47 Absolute increase of human cells in the CD40L culture over 14 days. Purified B cells of vaccinated (HBV) or PBMCs of non-vaccinated (control) donors were cultivated on CD40L-expressing $\mathrm{NIH}$ cells over a period of 14 days. The number of viable cells was determined every 3-4 days by trypan blue exclusion test. The absolute increase was determined by calculating the increase between two passages in percentage. Growth curve represent 3 independent donors.

Control cultures formed clusters on day 7 and day 11 with larger and evenly shapes on day 14 in correlation with their proliferative behavior (Fig. 3.48, left column).

HBV cultures formed round and evenly shaped cluster on day 7 , although magnetic beads from the purification process were still apparent (Fig. 3.48, right column). However, from day 11 on HBV cultures showed a less stable morphology with smaller and unevenly shaped clusters. 


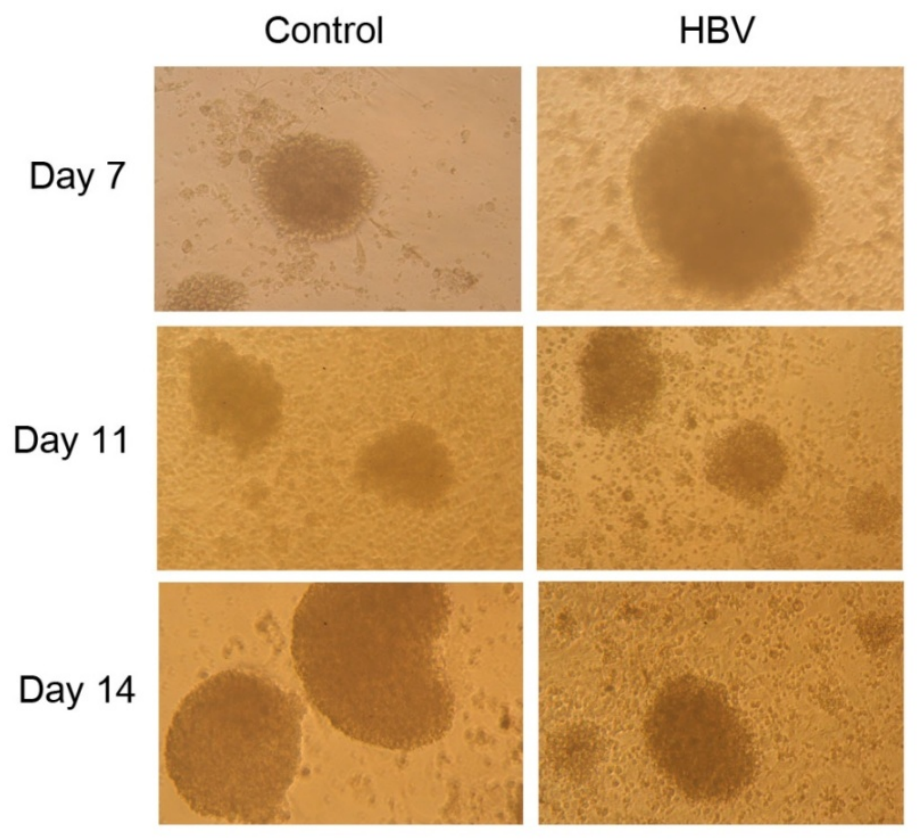

Fig. 3.48 Cluster formation of B cells in CD40L cultures. Purified B cells of vaccinated (HBV) or PBMCs of nonvaccinated (control) donors were cultivated on CD40L-expressing $\mathrm{NIH}$ cells over a period of 14 days. Pictures were taken on day 7, 11 and 14 at a 10x magnification using a light optical microscope fitted with a digital camera. Representative pictures of 3 independent experiments are shown.

Control and HBV cultures were analyzed by FACS for the percentage of $\mathrm{CD} 19^{+} \mathrm{CD} 20^{+} \mathrm{B}$ cells and the percentage of HBV-specific among the $\mathrm{CD} 19^{+} \mathrm{CD} 2 \mathrm{O}^{+} \mathrm{B}$ cell population. $\mathrm{B}$ cell purity constantly increased in control and HBV-specific cultures reaching a maximum on day 14 of $77 \%$ and $97 \%$ purity, respectively (Fig. 3.49). However, control cultures had started with a lower B cells purity than HBV-specific cultures. However, the percentage of HBV-specific cells among $\mathrm{CD} 19^{+} \mathrm{CD} 20^{+} \mathrm{B}$ cells decreased rapidly to about $1 \%$ on day 14 as observed in murine CD40B cultures (data not shown).

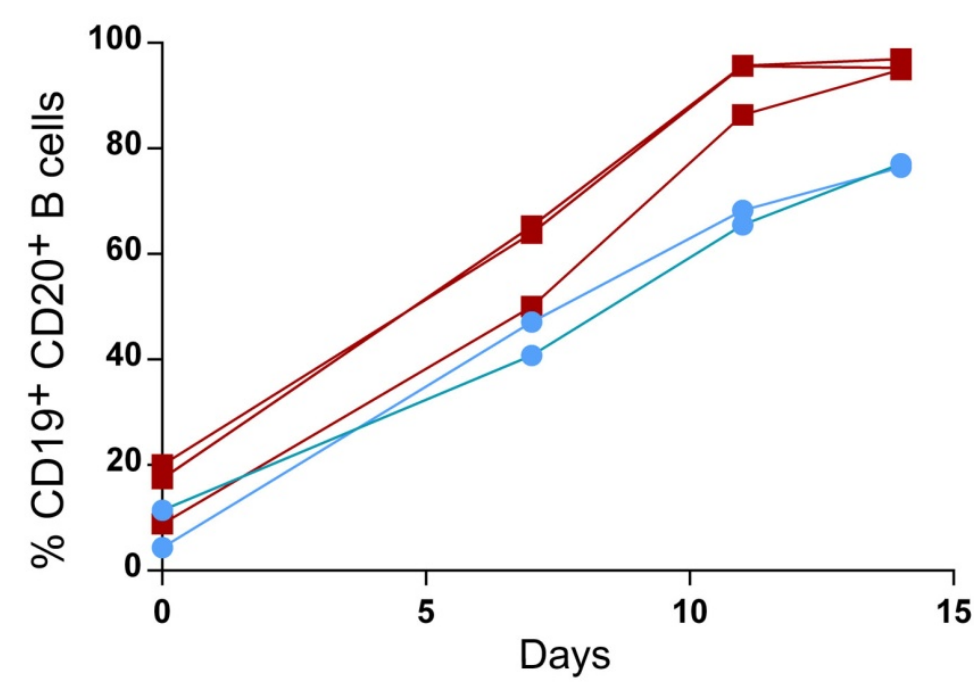

- Control CD40Bs donor 1

- Control CD40Bs donor 2

- HBV+ CD40Bs donor 3

HBV+ CD40Bs donor 4

$\mathrm{HBV}+\mathrm{CD} 40 \mathrm{Bs}$ donor 5

Fig. 3.49 Increase of CD19 ${ }^{+} \mathrm{CD} 2 \mathrm{O}^{+}$B cells in CD40L cultures. Purified B cells of vaccinated (HBV) or PBMCs of non-vaccinated (control) donors were cultivated on CD40L-expressing $\mathrm{NIH}$ cells over a period of 14 days. Staining for $\mathrm{CD}_{1} 9^{+} \mathrm{CD} 2 \mathrm{O}^{+} \mathrm{B}$ cells was performed on day $0,7,11$ and 14 and the percentage of $B$ cells was determined by flow cytometry analyses. Results of 5 independent donors are shown. 
On day 7, 11 and 14 CD40B cells were analyzed for the expression levels of several surface markers to confirm their antigen-presenting phenotype. The MFI of the molecules of interest was normalized to the MFI of their respective culture on day 0.

Interestingly, the expression of MHC II molecules (HLA-DR) in HBV-specific cultures was upregulated at first, but then decreased until day 14 to $59.60 \% \pm 7.66$ (Fig. 3.50, red and green line). Control cultures expressed significantly more HLA-DR on 14 days than HBVspecific cultures, although the total increase was only minor (107.85 \% \pm 6.66 ; Fig. 3.49 blue line).

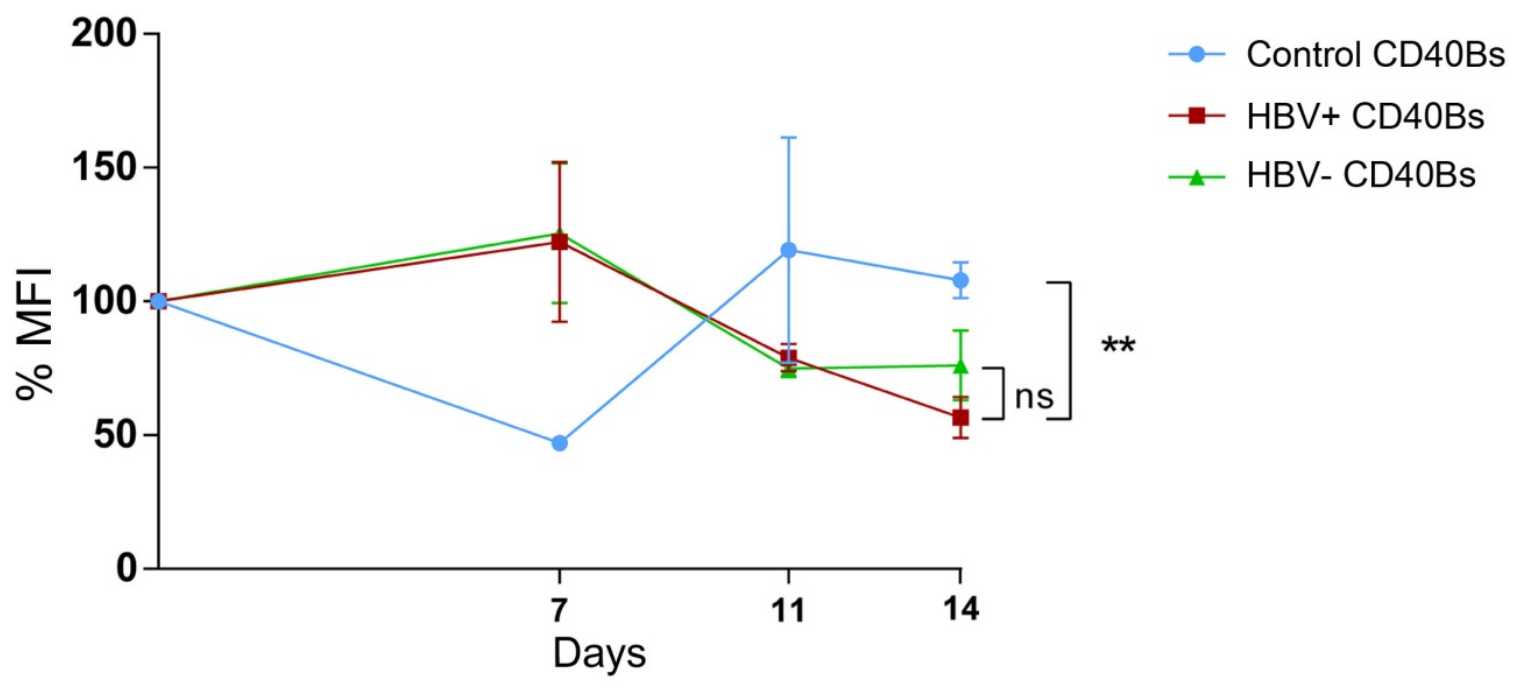

Fig. 3.50 MHC II expression in HBV-specific CD40B cells in the CD40 culture. The mean fluorescent intensity (MFI) of MHC II of Control CD40B cells, HBV-specific (HBV+) or HBV-negative (HBV-) CD4OB cells over a period of 14 days was determined by flow cytometry and values were normalized to the expression levels in $B$ cells of the respective populations on day 0 . Curves show mean values $\pm S D$ of three independent experiments. Significant differences were calculated with two-way ANOVA are marked by an asterisk. ${ }^{* *} \mathrm{p} \leq 0.01$.

The expression of the costimulatory molecules CD86 (Fig. 3.51, red and green line) was highly upregulated in HBV-specific CD40B cultures, although the expression decreased from day 11 to 14 . CD86 was also upregulated in control cultures (2024 \% \pm 114 ), although there was a significant difference to HBV-specific cultures (Fig. 3.51, blue line) 


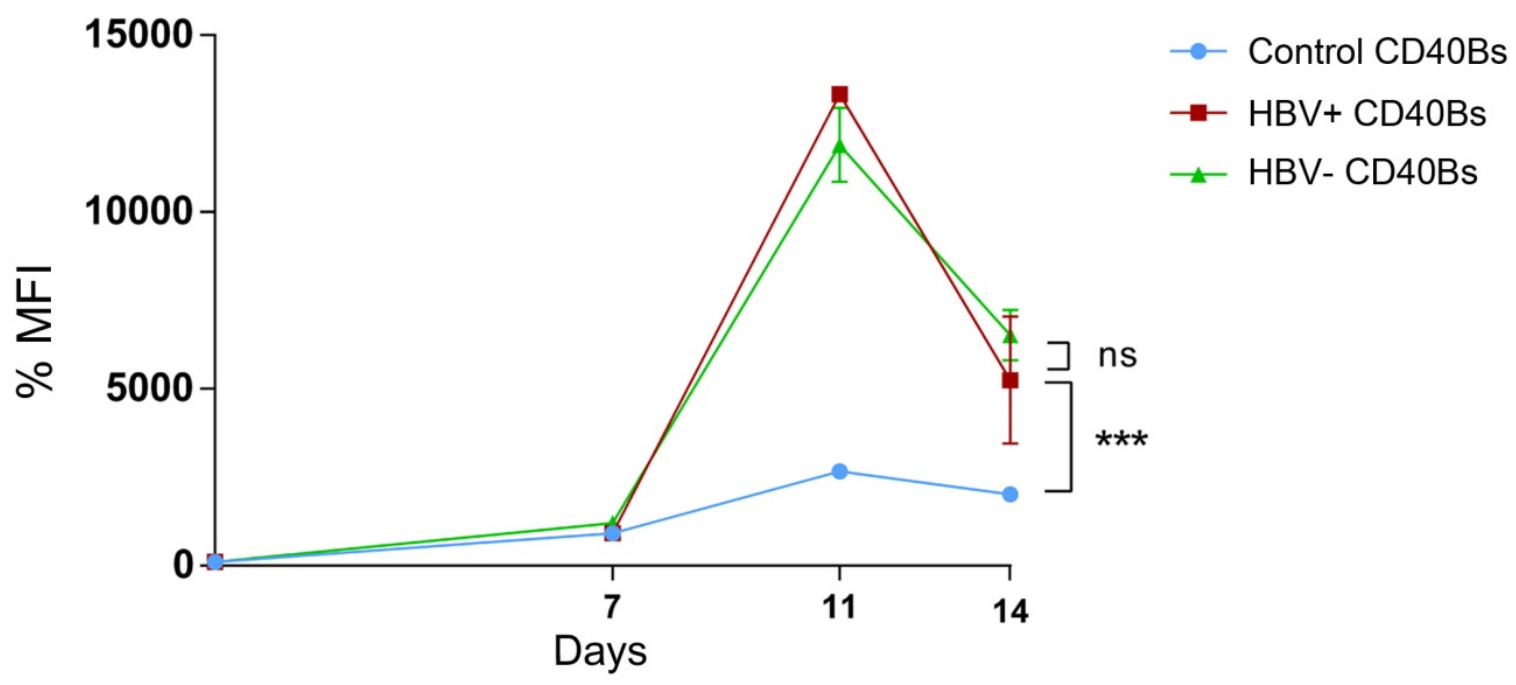

Fig. 3.51 CD86 expression in HBV-specific CD40B cells in the CD40 culture. The mean fluorescent intensity (MFI) of CD86 of Control CD40B cells, HBV-specific (HBV+) or HBV-negative (HBV-) CD4OB cells over a period of 14 days was determined by flow cytometry and values were normalized to the expression levels in $B$ cells of the respective populations on day 0 . Curves show mean values $\pm S D$ of three independent experiments. Significant differences were calculated with two-way ANOVA are marked by an asterisk. ${ }^{* * *} p \leq 0.001$.

HBV-specific CD40B cells $(3046 \% \pm 807)$ and control B cells $(2283 \% \pm 84)$ also highly upregulated the expression of CD80 (Fig. 3.52, red and blue line, respectively). Interestingly, the difference to HBV-negative CD4OB cells in HBV cultures on day 14 was highly significant (7760 \% \pm 594; Fig. 3.52, green line).

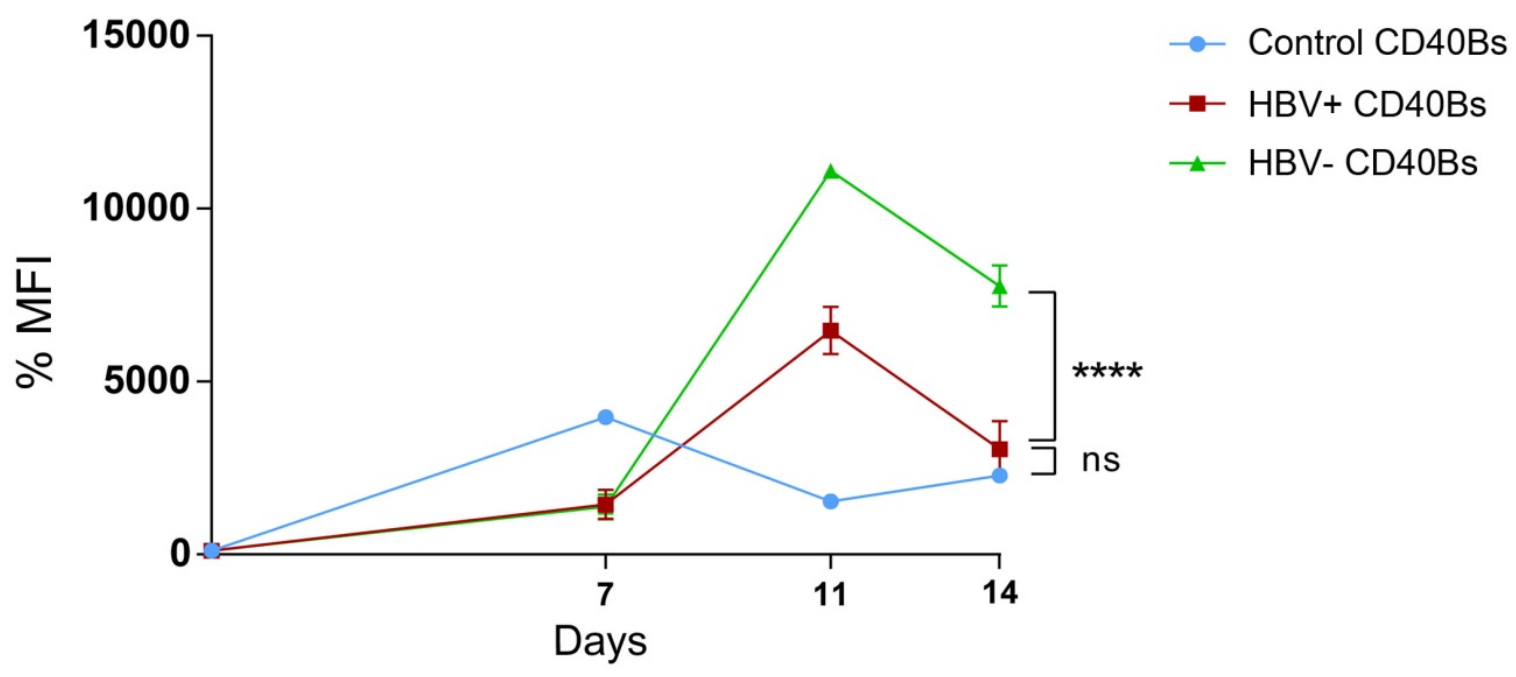

Fig. 3.52 CD80 expression in HBV-specific CD40B cells in the CD40 culture. The mean fluorescent intensity (MFI) of CD80 of Control CD40B cells, HBV-specific (HBV+) or HBV-negative (HBV-) CD4OB cells over a period of 14 days was determined by flow cytometry and values were normalized to the expression levels in $B$ cells of the respective populations on day 0 . Curves show mean values $\pm S D$ of three independent experiments. Significant differences were calculated with two-way ANOVA are marked by an asterisk. $* * * * p \leq 0.0001$. 
The percentage of CD138 ${ }^{+} \mathrm{HBV}$-negative and control CD40B cells decreased from day 0 until day 14 and there was no difference in the two populations (Fig. 3.53, green and blue line, respectively). However, the percentage of $\mathrm{CD} 138^{+} \mathrm{HBV}$-specific CD40B cells increased to $51.87 \% \pm 24.36$ until day 14 (Fig. 3.53, red line). The difference to HBV-negative and control CD40B cells was significant.

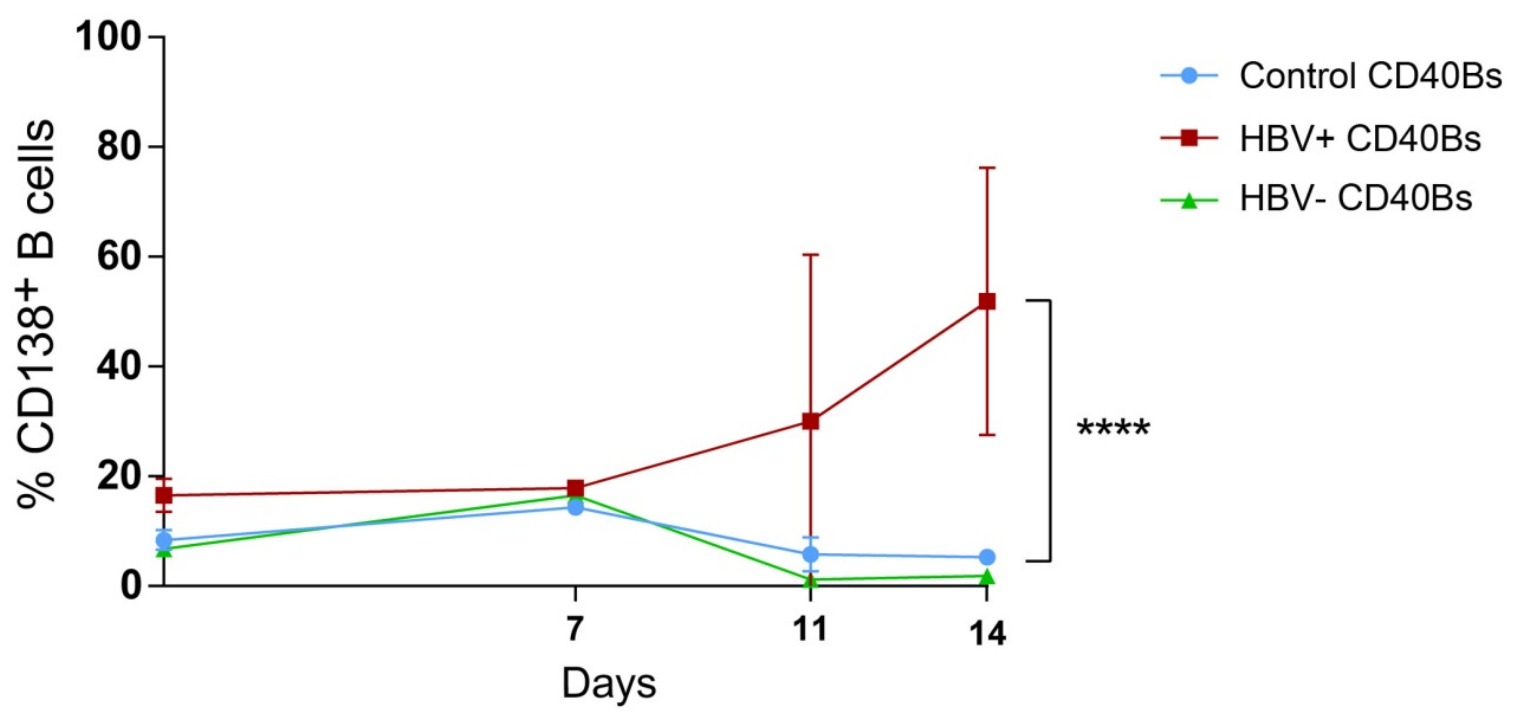

Fig. 3.53 Percentage of $\mathrm{CD} 138^{+} \mathrm{B}$ cells in the CD40 culture. The percentage of $\mathrm{CD} 138^{+} \mathrm{B}$ cells of Control CD4OB cells, HBV-specific (HBV+) or HBV-negative (HBV-) CD40B cells over a period of 14 days was determined by flow cytometry. Curves show mean values \pm SD of three independent experiments. Significant differences were calculated with two-way ANOVA are marked by an asterisk. $* * * * \mathrm{p} \leq 0.0001$.

\subsection{Murine Antigen-Specific CD40B Cells Efficiently Present Antigen to T Cells In Vitro}

To study the antigen-presenting function of murine OVA-specific CD4OB cells, their ability to stimulate an antigen-specific response of $\mathrm{CD}^{+}$or $\mathrm{CD}^{+} \mathrm{T}$ cells in vitro was investigated. For this purpose, a mixed-lymphocyte reaction (MLR) assay was performed (Steinman and Witmer 1978), in which protein-pulsed APCs are co-cultured together with $\mathrm{CD}^{+}$or $\mathrm{CD} 8^{+} \mathrm{T}$ cells from OT-II or OT-I mice, respectively. T cell activation and proliferation is induced via binding of the OVA-specific T-cell receptor to its antigen in the context of MHC presented on APCs. Co-stimulatory signals complete the activation of T cells through binding of CD80 (B71) and CD86 (B7-2) expressed on APCs to its receptor CD28 on the T cell surface (Galvin et al. 1992). 
OVA-specific B cells were purified from immunized C57BL/6 mice and activated in the CD40 system over night (OVA CD4OBs). In addition, they were incubated with 75 nM OVA-protein for 24 hours prior to incubation with T cells. Polyclonal B cells from naïve C57BL/6 mice served as control (control CD4OBs). The activation status of CD40B cells ( $80 \%$ B cell purity and $>60 \%$ OVA purity) was confirmed by analyzing the upregulation of the expression markers CD80, CD86, IA ${ }^{\mathrm{b}}$ (MHC II) and $\mathrm{H}_{2} \mathrm{~K}^{\mathrm{b}}$ (MHC I) by FACS (Fig. 3.42-3.45).

Bone-marrow derived dendritic cells served as alternative source of APCs and positive control in MLRs, since they have long been viewed as the most potent APCs (Inaba et al. 1990). DCs were generated from bone-marrow derived $C D 34^{+}$progenitor cells and matured by addition of anti-CD40 antibody or LPS (herein after referred to as CD40 DCs or LPS DCs, respectively). The two different stimuli were tested to cover the heterogeneity of DC subsets (Shortman and Liu 2002). The phenotype and activation status of mature DCs was confirmed by analyzing the $>90 \%$ pure $\mathrm{CD} 11 \mathrm{~b}^{+} \mathrm{CD} 11 \mathrm{c}^{+}$population (Fig. 3.54) for their upregulation of the activation markers CD80, CD83, CD86 and IA ${ }^{\text {b }}$ by FACS (Fig. 3.55).
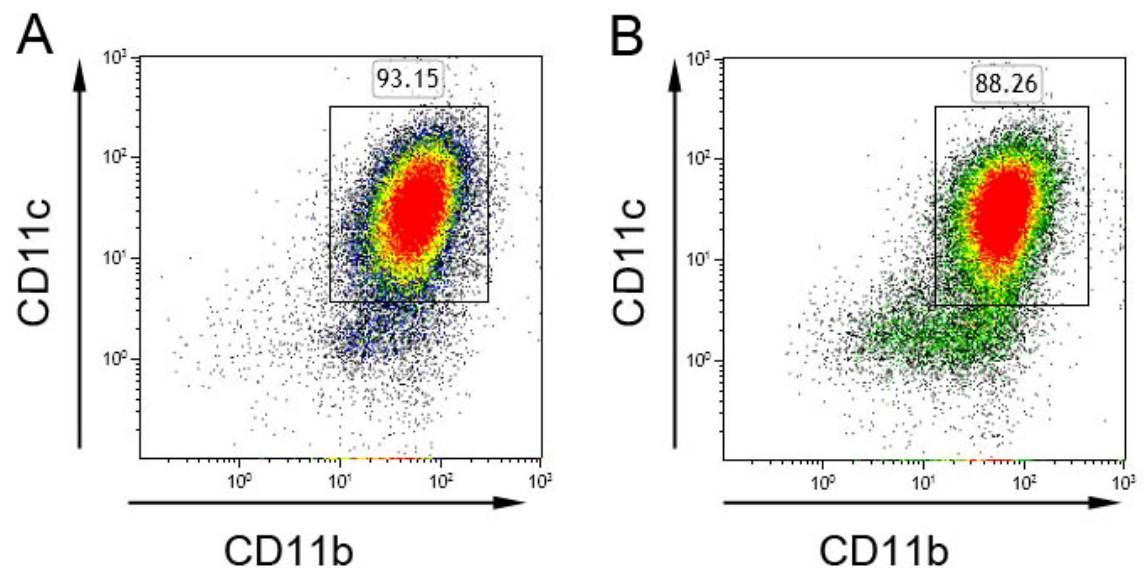

Fig. 3.54 Purity of matured CD40 DCs and LPS DCs. Representative flow cytometry analyses of CD40L-matured DCs (CD40 DCs, A) and LPS-matured DCs (LPS DCs, B) out of at least 5 independent experiments are shown. Mature DCs were stained for $\mathrm{CD}_{11} \mathrm{~b}^{+} \mathrm{CD} 11 \mathrm{c}^{+}$cells. Numbers indicate the percentages. 

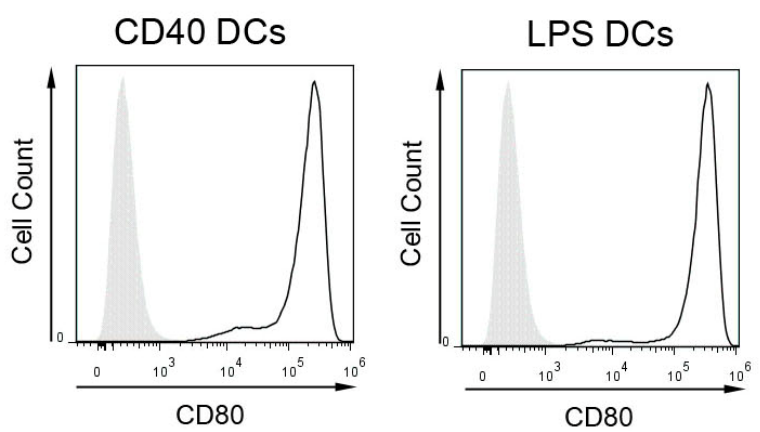

Fig. 3.55 Expression of activation markers in mature DCs. Representative flow cytometry analyses of CD40L-matured DCs (CD40 DCs, A) and LPS-matured DCs (LPS DCs, B) out of at least 5 independent experiments are shown. Mature DCs were stained for the activation markers CD80, CD83, CD86 and IAb were indicated (black line). Unstained cells served as control
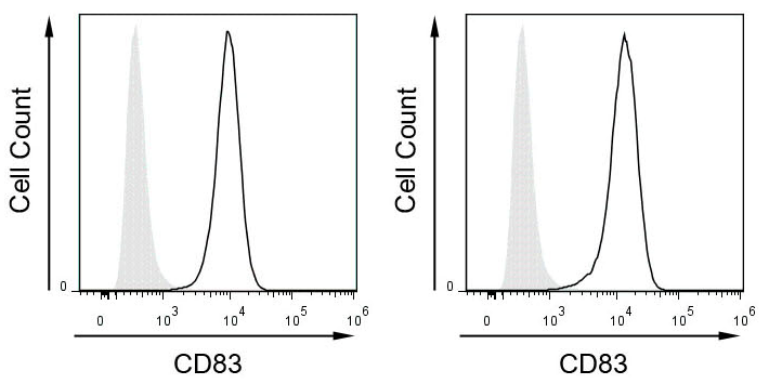
(filled line).
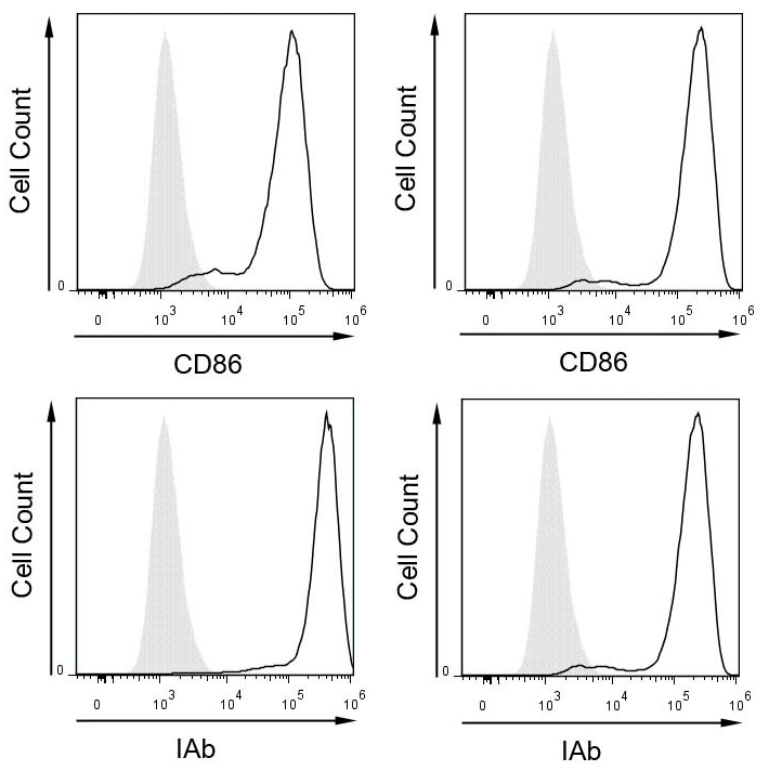

Antigen-specific $\mathrm{CD}^{+}$or $\mathrm{CD}^{+} \mathrm{T}$ cells were isolated from OT-II or OT-I mice, respectively. Antigen-specificity of the $>90 \% \mathrm{CD}^{+} \mathrm{T}$ cell population (Fig. 3.56, upper row) was determined by analyzing the expression of TCR-chain VB5 on $\mathrm{CD}^{+}$or $\mathrm{CD} 8^{+} \mathrm{T}$ cells by FACS (Fig. 3.56, lower row). 

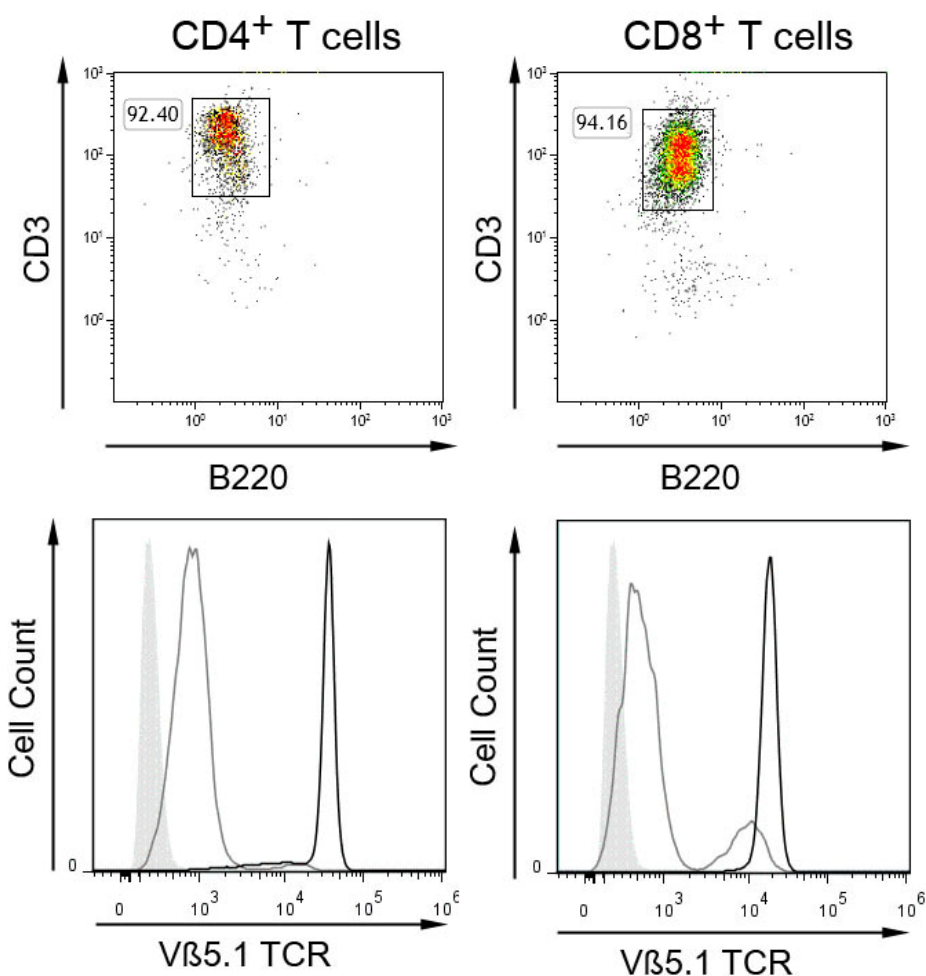

Fig. 3.56 Purity of OT-I and OT-II T cells. Representative flow cytometry analyses of OT-II (CD4 ${ }^{+} \mathrm{T}$ cells, left column) and OT-II (CD8 ${ }^{+} \mathrm{T}$ cells, right column) $T$ cells out of at least 5 independent experiments are shown. Purified T cells were stained for $\mathrm{CD}^{+}{ }^{+} \mathrm{B}^{2} 20^{-}$cells (upper row) and their expression of $V \beta 5.1$ TCR (lower row, black line). Numbers indicate the percentages. T cells from C57BL/6 mice served as negative controls (grey line) and unstained cells as staining control (filled line).

T cells were labeled with CFSE to measure their proliferation and were cultured together with control CD40Bs (with or without protein), OVA CD40Bs (with or without protein), CD40 DCs or LPS DCs at various APC-to-T cell ratios $(0: 1,3: 1,1: 1,1: 5,1: 10,1: 20,1: 50)$ for 5 days. CFSE segregates equally between daughter cells upon cell division. When analyzed by FACS, this sequential halving of fluorescence is visualized as distinct peaks in the histogram (Fig. 3.58).

Incubation of either $\mathrm{CD}^{+}$or $\mathrm{CD}^{+} \mathrm{T}$ cells with protein-pulsed control CD40Bs in a ratio 1:1 resulted in proliferation of a small proportion of T cells $(8.43 \% \pm 3.45$ and $4.61 \% \pm 2.45$, respectively; Fig. 3.57 A and B). However, this induction of proliferation was not significantly higher than in non-pulsed control CD40Bs. Cocultures of protein-pulsed OVA CD40Bs with either $\mathrm{CD}^{+}$or $\mathrm{CD}^{+} \mathrm{T}$ cells in a ratio of 1:1 resulted in high proliferation of the T cells $(61.56$ $\% \pm 12.40$ and $72.66 \% \pm 14.15$, respectively). Although protein-pulsed OVA CD4OBs induced more proliferation than OVA CD4OBs that received no additional OVA-protein $(38.18 \% \pm$ 19.57 and $50.77 \% \pm 33.07$, respectively), the difference was not significant. Moreover, culture of T cells with CD40 DCs or LPS DCs in a ratio of 1:1 induced high proliferation of T cells. Proliferation of $\mathrm{CD}^{+}$or $\mathrm{CD} 8^{+} \mathrm{T}$ cells was higher in CD40 DCs $(77.11 \% \pm 6.02$ and 97.62 $\% \pm 1.09$, respectively) than in LPS DCs $(68.55 \% \pm 1.41$ and $76.85 \% \pm 19.13$, respectively). However, the difference was not significant. Furthermore, there was no significant 
difference in induction of T cell proliferation between OVA CD40Bs and the two DC subtypes, but proliferation was significantly lower in cocultures with control CD4OBs than with OVA CD40Bs or DCs.
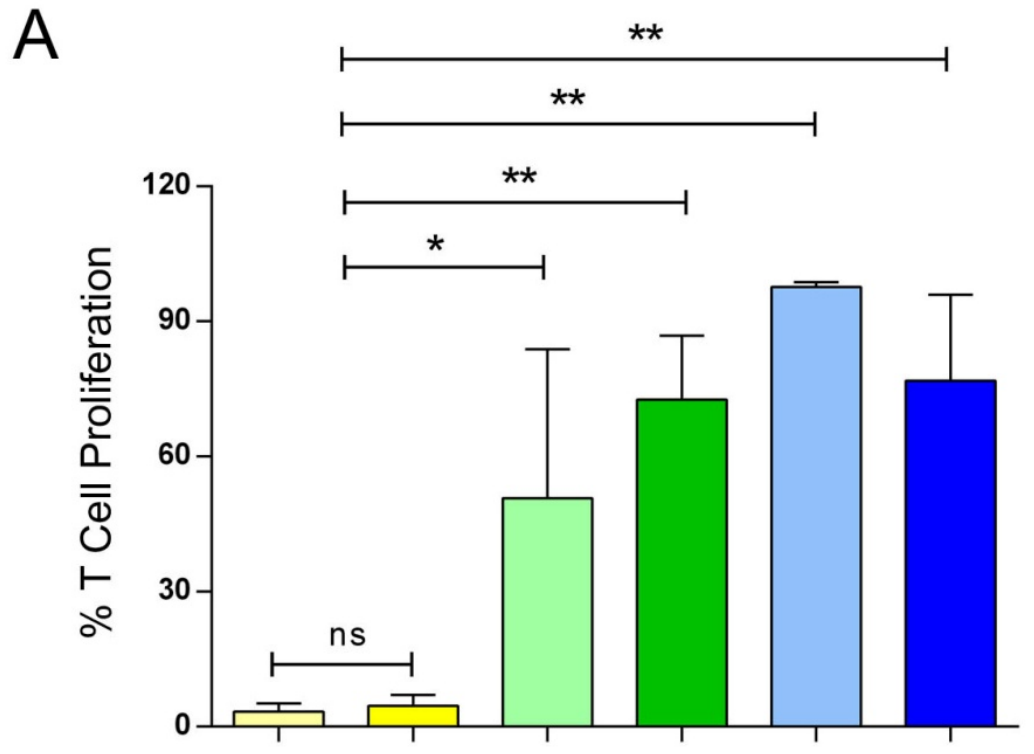

Ratio 1:1

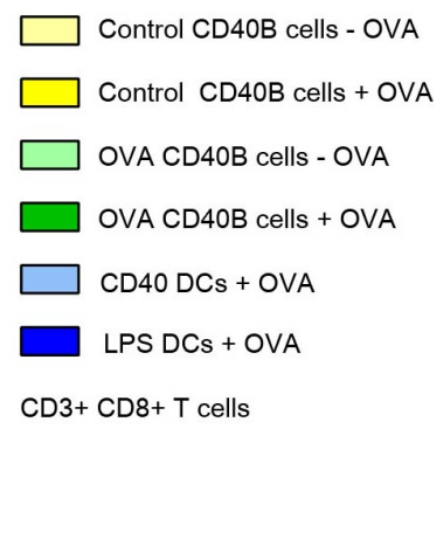

B

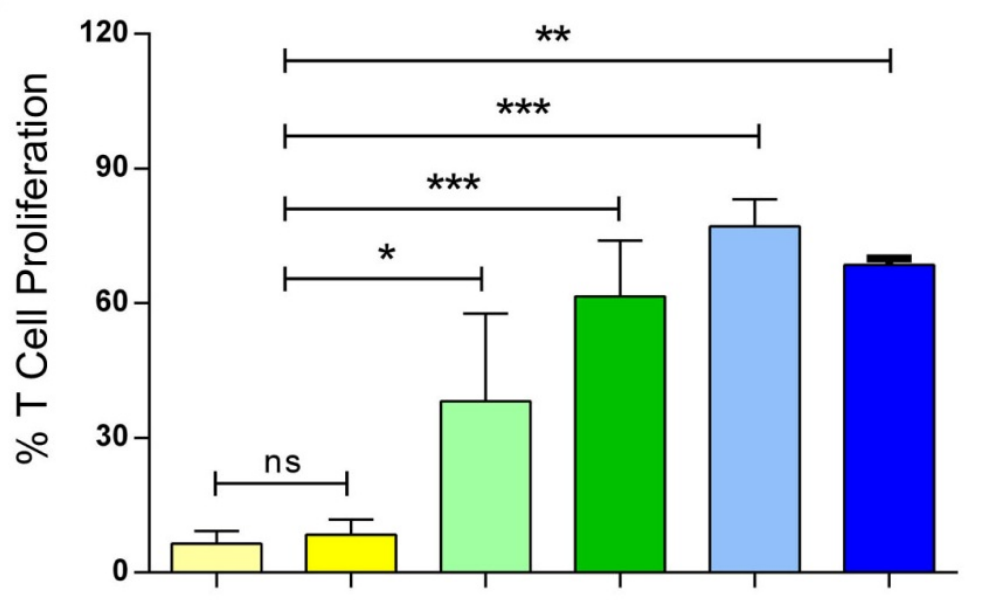

Ratio 1:1

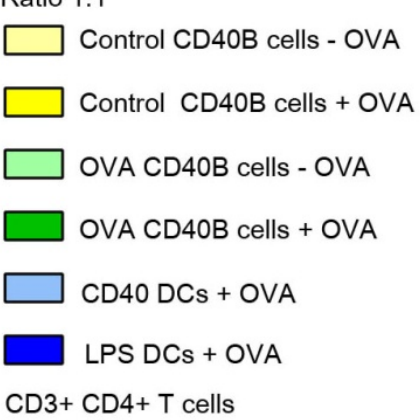

Fig. 3.57 T cell proliferation in co-cultures of APCs and OT-II or OT-I T cells in the ratio 1:1. Polyclonal CD40B cells (Control CD40B cells), OVA-specific CD40B cells (OVA CD40B cells) CD40matured DCs (CD40 DCs) or LPS-matured DCs (LPS DCs) were left untreated (- OVA) or pulsed with protein (+ OVA) over night. Afterwards, they were mixed with OT-I (A) or OT-II (B) T cells in a ratio of 1:1 and incubated for 5 days. Proliferation of T cells was determined by flow cytometry by a decrease in CFSE labeling. Bar charts represent mean values \pm SD of 4 independent experiments. Significant differences were calculated with two-way ANOVA are marked by an asterisk. ${ }^{*} p \leq 0.05,{ }^{* *} p \leq 0.01$, $* * * \mathrm{p} \leq 0.001$.

Performing four independent MLRs, it was observed that induction of proliferation by peptide-pulsed OVA CD4OBs is dependent on APC-to-T-cell ratio. Increasing the ratio to 3:1 induced even more proliferation of $\mathrm{CD}^{+}$or $\mathrm{CD}^{+} \mathrm{T}$ cells $(77.66 \% \pm 11.01$ and $80.99 \% \pm$ 
17.17, respectively; Fig. 3.58 and Fig. 3.59). By decreasing the APC-to-T-cell ratio to $1: 5$ the differences between protein-pulsed OVA CD4OBs cells $\left(29.46 \% \pm 12.97\right.$ for CD4 ${ }^{+} \mathrm{T}$ cells and $34.12 \% \pm 20.15$ for $\mathrm{CD}^{+}$T cells; Fig. 3.60) and OVA CD40Bs that did not receive additional protein (14.20\% \pm 9.68 for $\mathrm{CD}^{+} \mathrm{T}$ cells and $5.76 \% \pm 3.66$ for $\mathrm{CD}^{+} \mathrm{T}$ cells) became more obvious, although it was not significant. Induction of proliferation further decreased in an OVA CD40Bs-to-T-cell ratio of 1:10 $\left(7.81 \% \pm 4.53\right.$ for $\mathrm{CD}^{+} \mathrm{T}$ cells and $8.90 \% \pm 4.50$ for $\mathrm{CD} 8^{+}$ T cells; Fig. 3.61) and reduced to almost zero in ratios of 1:20 and 1:50 (data not shown). CD40 DCs or LPS DCs were less affected by lower APCs-to-T-cell ratios. In CD40 DC cocultures at a ratio of 1:10 induction of proliferation was still significantly higher than in control CD40Bs (53.06 \% \pm 25.26 for $\mathrm{CD}^{+} \mathrm{T}$ cells and $89.40 \% \pm 4.06$ for $\mathrm{CD}^{+} \mathrm{T}$ cells; Fig. 3.61). Although induction of proliferation was lower in LPS DC cocultures $\left(33.82 \% \pm 0.40\right.$ for $C D 4^{+} T$ cells and $42.54 \% \pm 29.71$ for $\mathrm{CD}^{+} \mathrm{T}$ cells) the difference to control CD40Bs was significant. Even at low DC-to-T-cell ratios of 1:20 and 1:50, proliferation was still detected, although differences to control CD40Bs were not significant anymore (data not shown). 

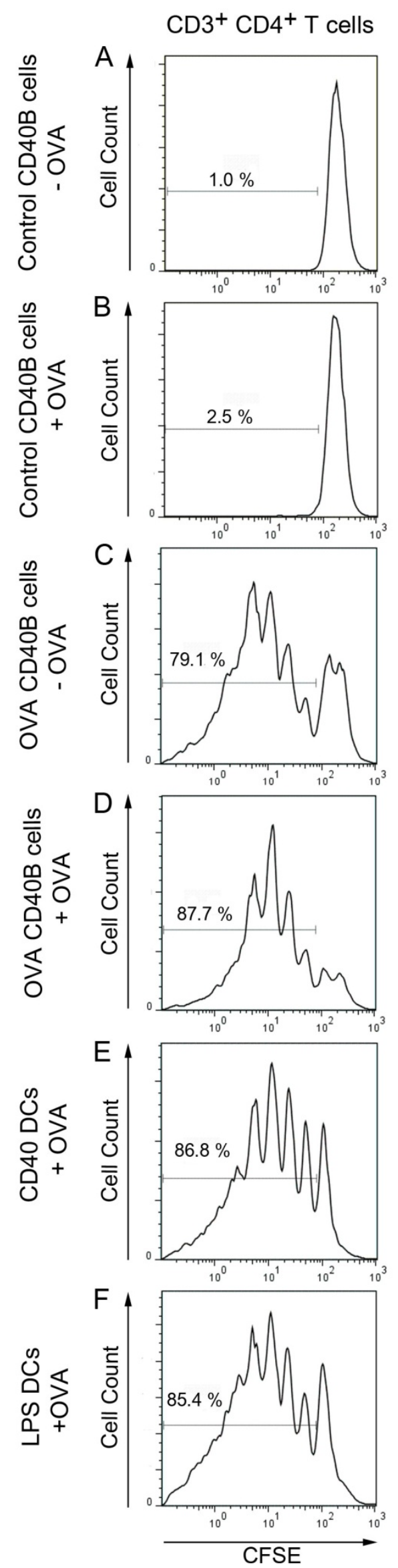

$\mathrm{CD}^{+}{ }^{+} \mathrm{CD}^{+}{ }^{+} \mathrm{T}$ cells
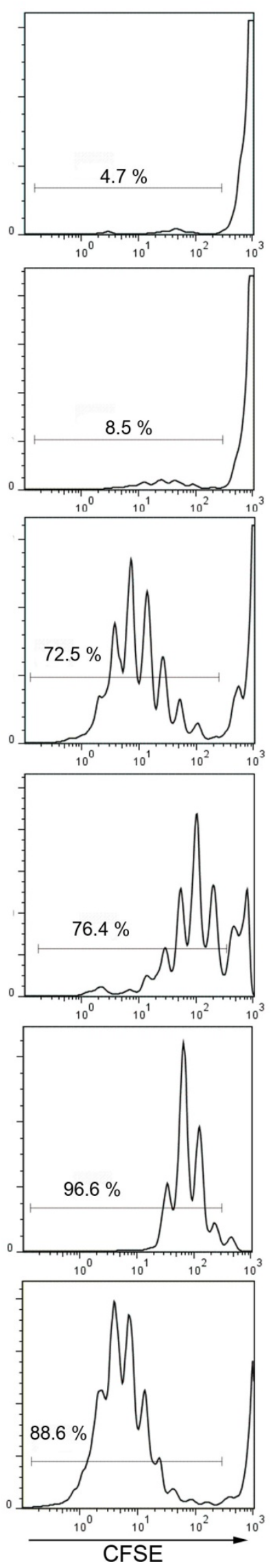

Fig. $3.58 \mathrm{~T}$ cell proliferation in co-cultures of APCs and OT-II or OT-I T cells in the ratio 3:1. Polyclonal CD40B cells (control CD40B cells), OVA-specific CD40B cells (OVA CD40B cells) CD40-matured DCs (CD40 DCs) or LPS-matured DCs (LPS DCs) were left untreated (- OVA) or pulsed with protein (+ OVA) over night. Afterwards, they were mixed with OT-I (left column) or OT-II (right column) $T$ cells in a ratio of 3:1 and incubated for 5 days. Proliferation of $T$ cells was determined by flow cytometry by a decrease in CFSE labeling. Representative flow cytometry histograms out of 4 independent experiments are shown. Numbers indicate percentage of proliferated T cells. 


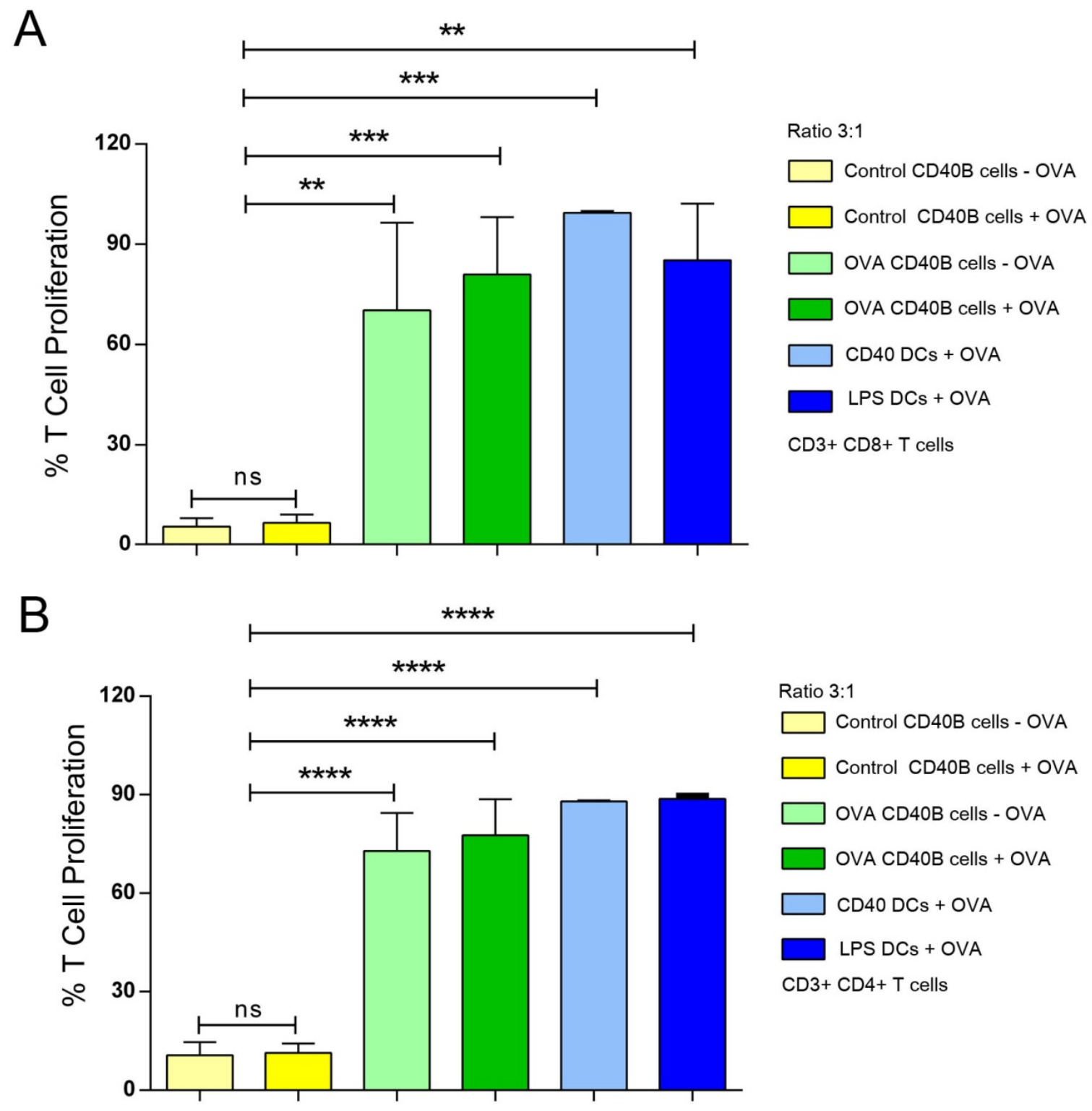

Fig. 3.59 T cell proliferation in co-cultures of APCs and OT-II or OT-I T cells in the ratio 3:1. Polyclonal CD40B cells (control CD40B cells), OVA-specific CD40B cells (OVA CD40B cells) CD40matured DCS (CD40 DCs) or LPS-matured DCs (LPS DCs) were left untreated (- OVA) or pulsed with protein (+ OVA) over night. Afterwards, they were mixed with OT-I (A) or OT-II (B) T cells in a ratio of 3:1 and incubated for 5 days. Proliferation of T cells was determined by flow cytometry by a decrease in CFSE labeling. Bar charts represent mean values \pm SD of 4 independent experiments. Significant differences were calculated with two-way ANOVA are marked by an asterisk. ${ }^{* *} p \leq 0.01, * * * p \leq$ $0.001, * * * * \mathrm{p} \leq 0.0001$. 


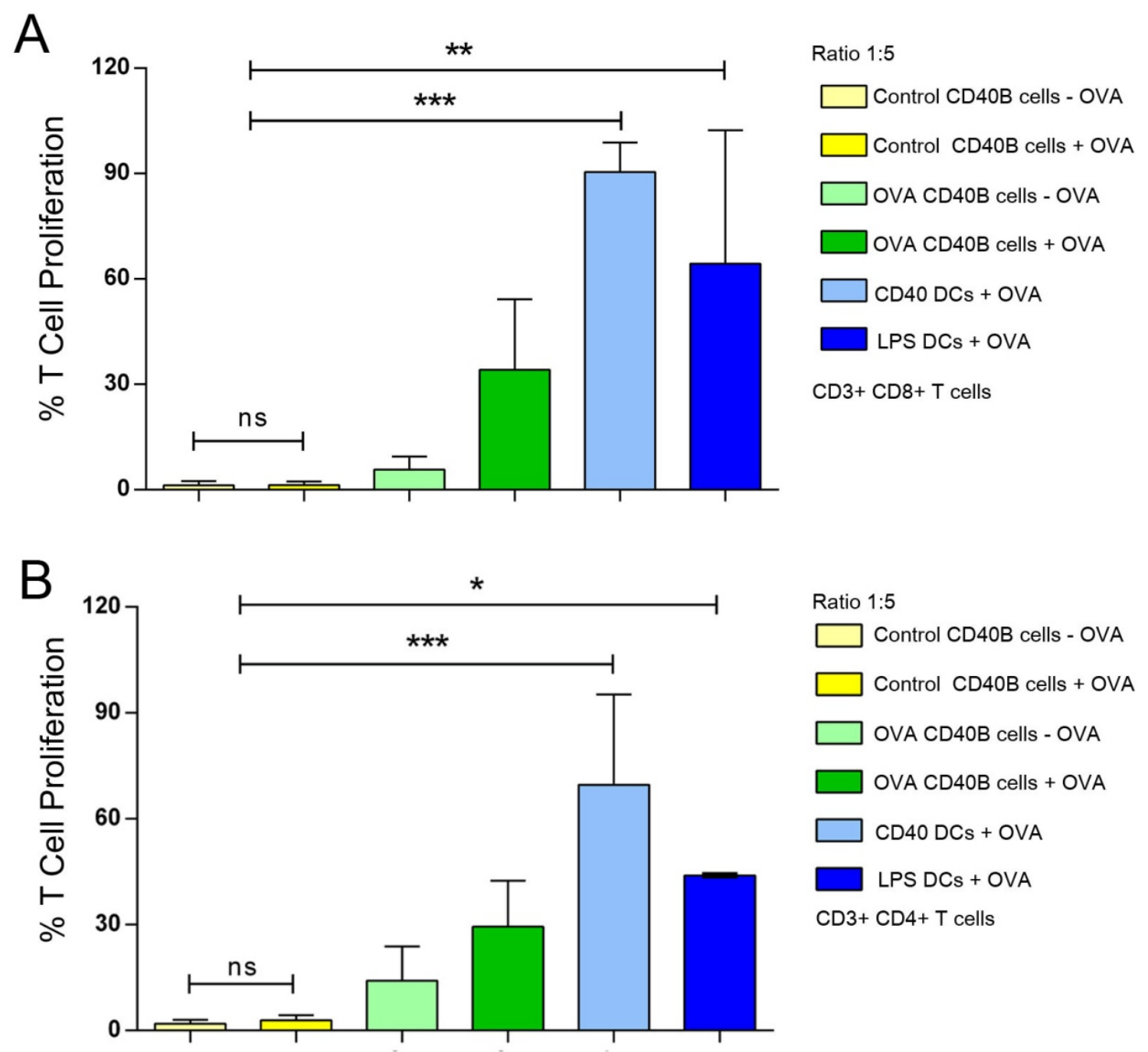

Fig. 3.60 T cell proliferation in co-cultures of APCs and OT-II or OT-I T cells in the ratio 1:5. Polyclonal CD40B cells (control CD40B cells), OVA-specific CD40B cells (OVA CD40B cells) CD40matured DCs (CD40 DCs) or LPS-matured DCs (LPS DCs) were left untreated (- OVA) or pulsed with protein (+ OVA) over night. Afterwards, they were mixed with OT-I (A) or OT-II (B) T cells in a ratio of 1:5 and incubated for 5 days. Proliferation of T cells was determined by flow cytometry by a decrease in CFSE labeling. Bar charts represent mean values \pm SD of 4 independent experiments. Significant differences were calculated with two-way ANOVA are marked by an asterisk. ${ }^{*} p \leq 0.05,{ }^{* *} p \leq 0.01$, $* * * \mathrm{p} \leq 0.001$. 


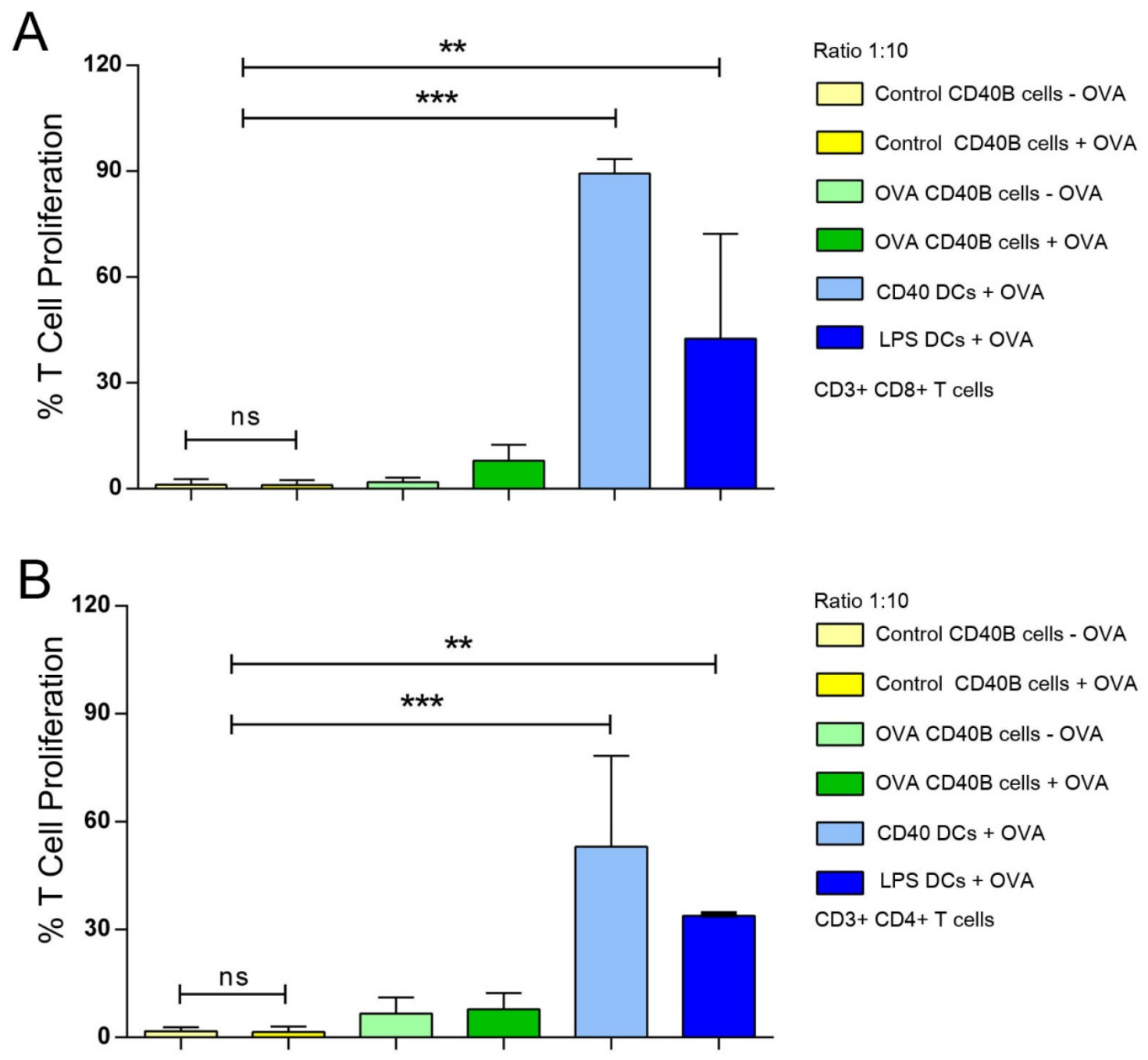

Fig. 3.61 T cell proliferation in co-cultures of APCs and OT-II or OT-I T cells in the ratio 1:10. Polyclonal CD40B cells (control CD40B cells), OVA-specific CD40B cells (OVA CD40B cells) CD40matured DCs (CD40 DCs) or LPS-matured DCs (LPS DCs) were left untreated (- OVA) or pulsed with protein (+ OVA) over night. Afterwards, they were mixed with OT-I (A) or OT-II (B) T cells in a ratio of 1:10 and incubated for 5 days. Proliferation of $T$ cells was determined by flow cytometry by a decrease in CFSE labeling. Bar charts represent mean values \pm SD of 4 independent experiments. Significant differences were calculated with two-way ANOVA are marked by an asterisk. ${ }^{* *} p \leq 0.01$, $* * * p \leq 0.001$.

In addition, stimulation of T cell proliferation by either source of APC was accompanied by an upregulation CD25 expression indicating T cell activation (Fig. 3.62- 3.65). In cocultures of OVA CD40Bs and $\mathrm{CD}^{+}$or $\mathrm{CD} 8^{+}$T cells, upregulation of CD25 was high in ratios 3:1 $154.93 \% \pm$ 20.57 for $\mathrm{CD}^{+} \mathrm{T}$ cells and $85.54 \% \pm 5.80$ for $\mathrm{CD} 8^{+} \mathrm{T}$ cells; Fig. 3.62$)$ and $1: 1(46.45 \% \pm 16.39$ for $\mathrm{CD}^{+} \mathrm{T}$ cells and $72.64 \% \pm 11.95$ for $\mathrm{CD}^{+} \mathrm{T}$ cells; Fig. 3.63) and decreased in lower ratios (Fig. 3.64 and Fig. 3.65 and data not shown) according to T cell proliferation. In ratios 3:1 and $1: 1$, the differences to control CD40Bs cells were significant and the influence of additional protein pulsing became more obvious than in T cell proliferation. Although in cocultures with 
control CD40Bs upregulation of $\mathrm{CD} 25$ was detected in $\mathrm{CD}^{+} \mathrm{T}$ cells in ratios 3:1 and 1:1 (3.42 $\% \pm 2.70$ and $2.27 \% \pm 2.03$, respectively), it dropped to almost zero in lower control CD40Bsto- $\mathrm{CD}^{+}{ }^{+}$-cell ratios (Fig. 3.64 and Fig. 3.65, and data not shown). $\mathrm{CD}^{+}$or $\mathrm{CD}^{+}{ }^{+}$cell activation in a 3:1 ratio was high in CD40 DCs $(60.72 \% \pm 20.48$ and $84.54 \% \pm 5.80$, respectively) and LPS DCs (56.54 \% \pm 5.78 and $71.15 \% \pm 26.42$, respectively;) and remained above $10 \%$ in all testes ratios.

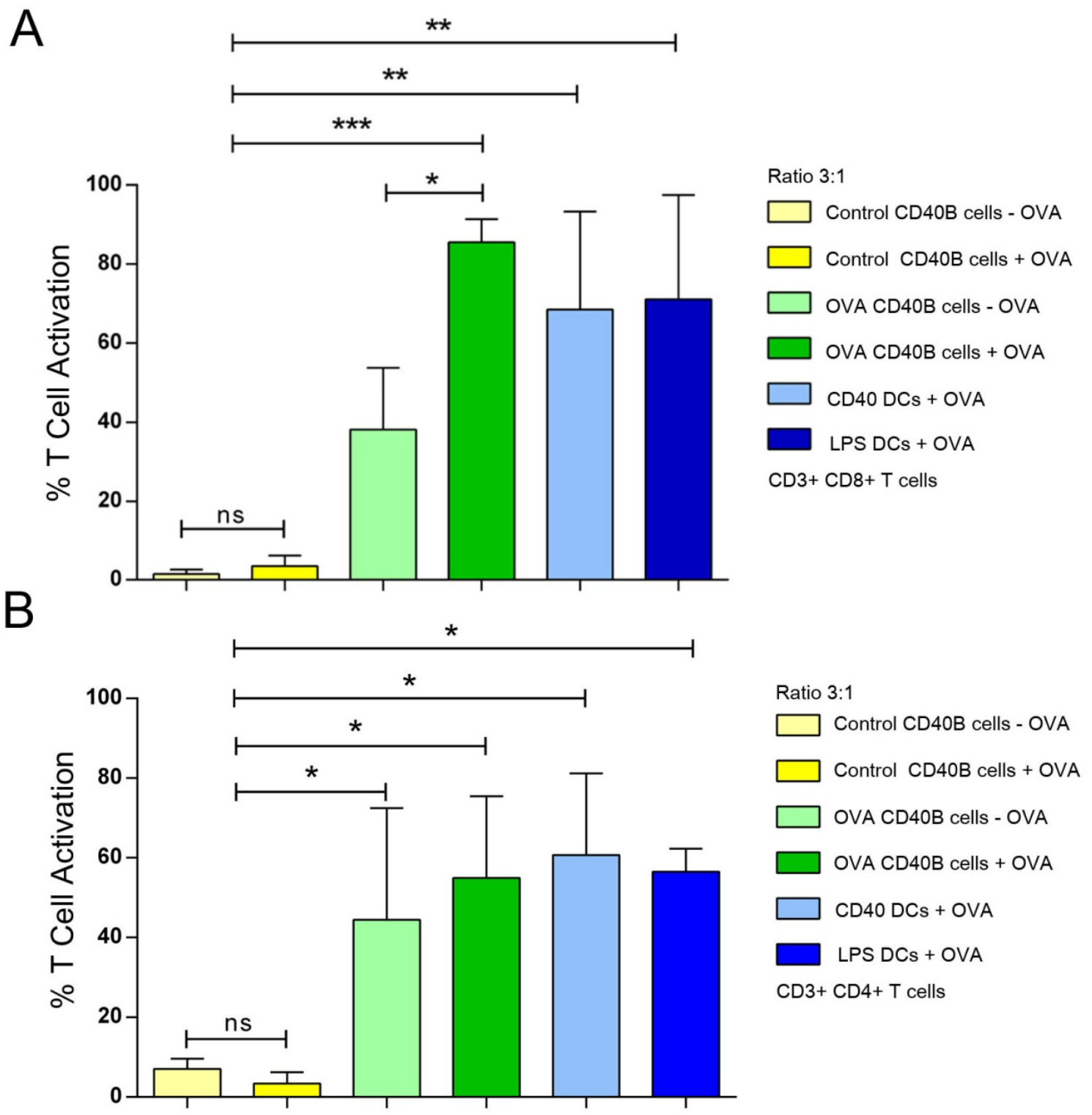

Fig. 3.62 T cell activation in co-cultures of APCs and OT-II or OT-I T cells in the ratio 3:1. Polyclonal CD40B cells (control CD40B cells), OVA-specific CD40B cells (OVA CD40B cells) CD40-matured DCs (CD40 DCs) or LPS-matured DCs (LPS DCs) were left untreated (- OVA) or pulsed with protein (+ OVA) over night. Afterwards, they were mixed with OT-I (A) or OT-II (B) T cells in a ratio of 3:1 and incubated for 5 days. Activation of $T$ cells was determined by staining for CD25 expression and subsequent flow cytometry analysis. Bar charts represent mean values \pm SD of 4 independent 
experiments. Significant differences were calculated with two-way ANOVA are marked by an asterisk. $* p \leq 0.05, * * p \leq 0.01, * * * p \leq 0.001$.

A

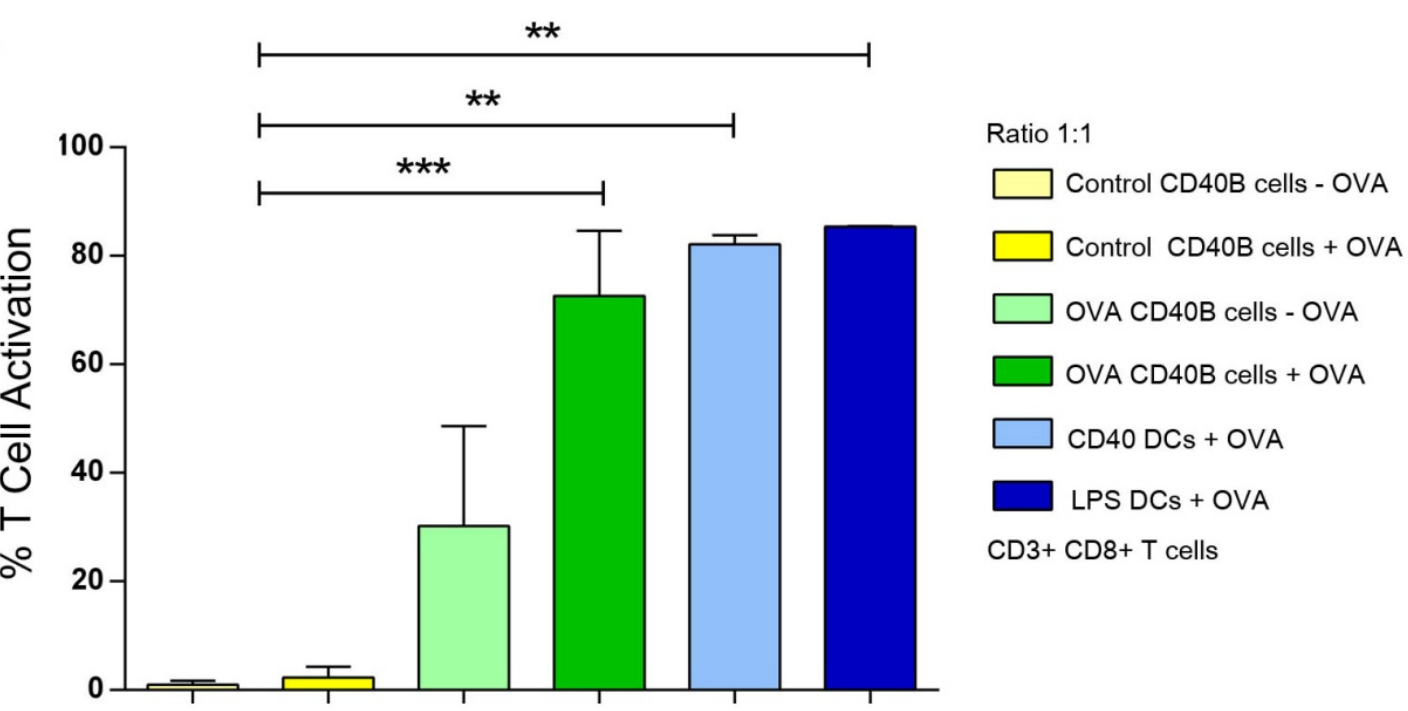

B

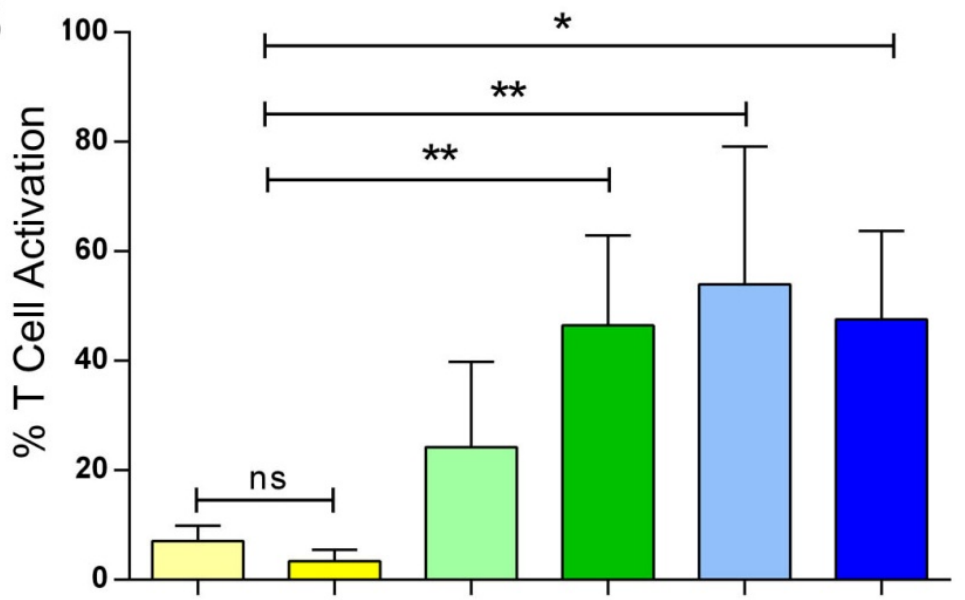

Ratio 1:1

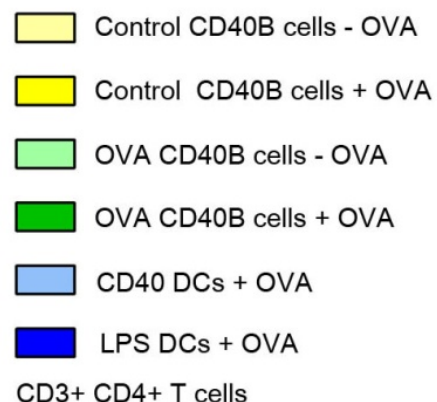

Fig. 3.63 T cell activation in co-cultures of APCs and OT-II or OT-I T cells in the ratio 1:1. Polyclonal CD40B cells (control CD40B cells), OVA-specific CD40B cells (OVA CD40B cells) CD40-matured DCs (CD40 DCs) or LPS-matured DCs (LPS DCs) were left untreated (- OVA) or pulsed with protein (+ OVA) over night. Afterwards, they were mixed with OT-I (A) or OT-II (B) T cells in a ratio of 1:1 and incubated for 5 days. Activation of T cells was determined by staining for CD25 expression and subsequent flow cytometry analysis. Bar charts represent mean values \pm SD of 4 independent experiments. Significant differences were calculated with two-way ANOVA are marked by an asterisk. $* p \leq 0.05, * * p \leq 0.01, * * * p \leq 0.001$. 

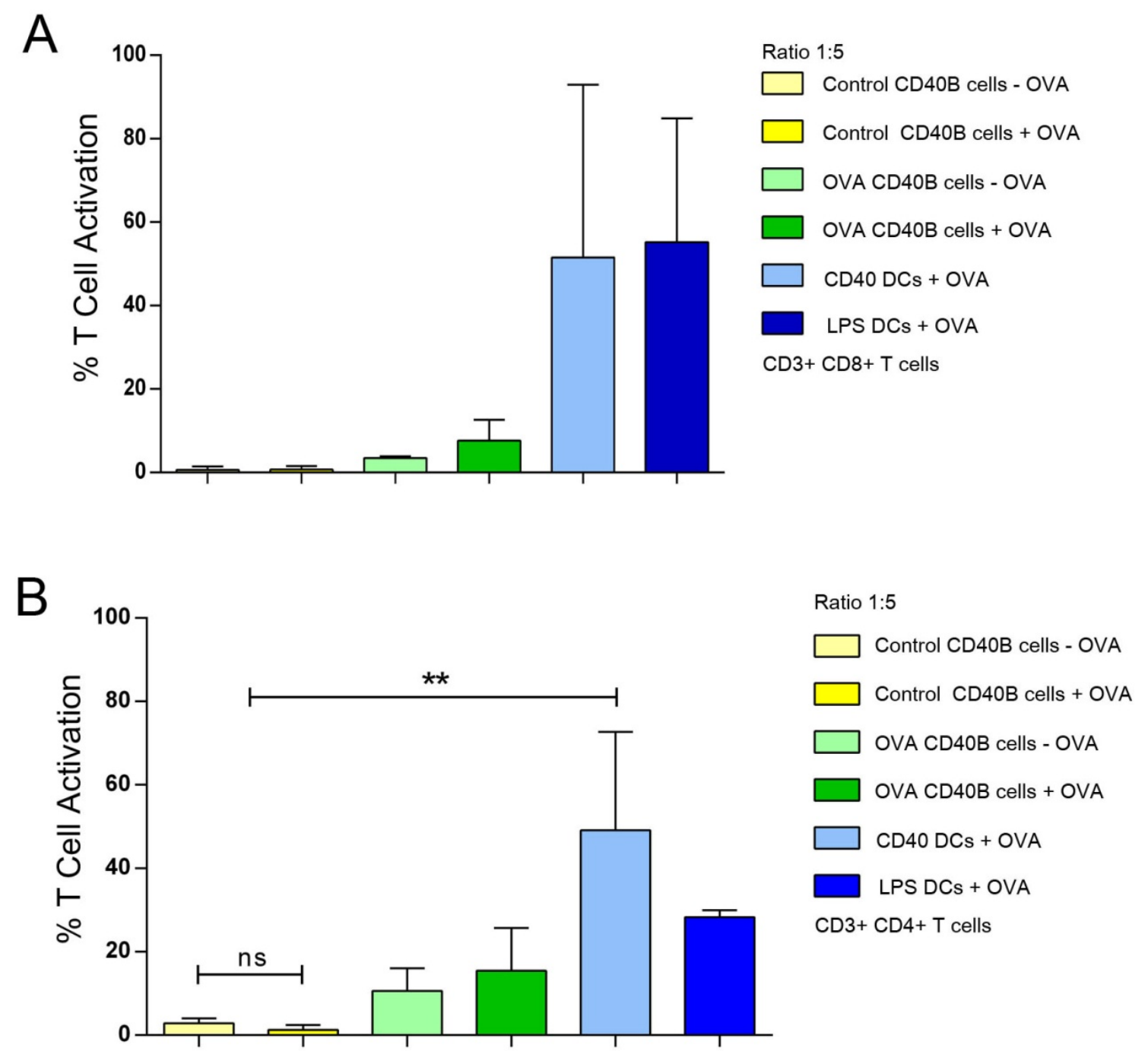

Fig. 3.64 T cell activation in co-cultures of APCs and OT-II or OT-I T cells in the ratio 1:5. Polyclonal CD40B cells (control CD40B cells), OVA-specific CD40B cells (OVA CD40B cells) CD40-matured DCs (CD40 DCs) or LPS-matured DCs (LPS DCs) were left untreated (- OVA) or pulsed with protein (+ OVA) over night. Afterwards, they were mixed with OT-I (A) or OT-II (B) T cells in a ratio of 1:5 and incubated for 5 days. Activation of T cells was determined by staining for CD25 expression and subsequent flow cytometry analysis. Bar charts represent mean values \pm SD of 4 independent experiments. Significant differences were calculated with two-way ANOVA are marked by an asterisk. $* * p \leq 0.01$. 
A

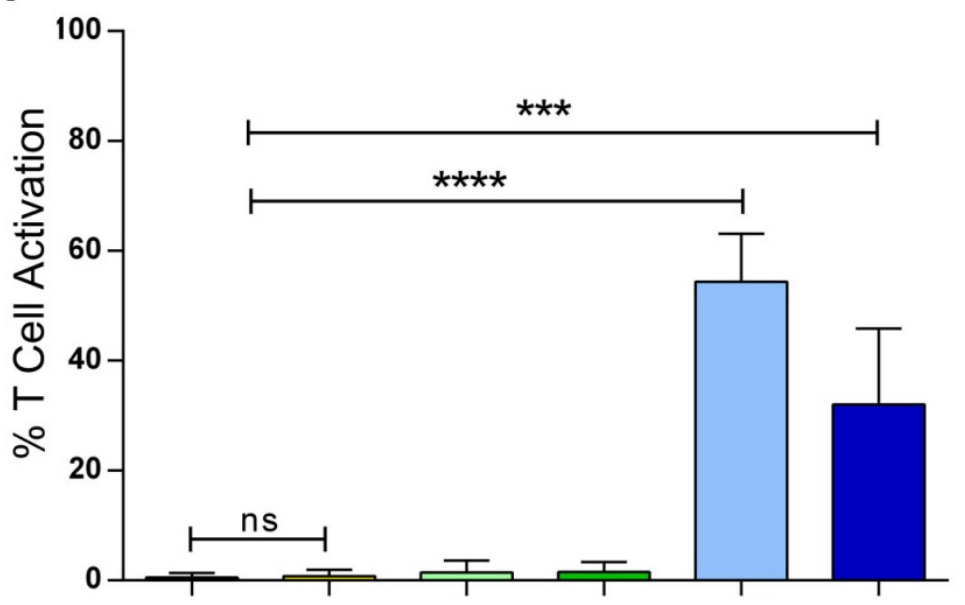

Ratio 1:10

Control CD4OB cells - OVA

$\square$ Control CD40B cells + OVA

$\square$ OVA CD40B cells - OVA

$\square$ OVA CD40B cells + OVA

CD40 DCs + OVA

LPS DCs + OVA

$\mathrm{CD} 3+\mathrm{CD} 8+\mathrm{T}$ cells

\section{B}

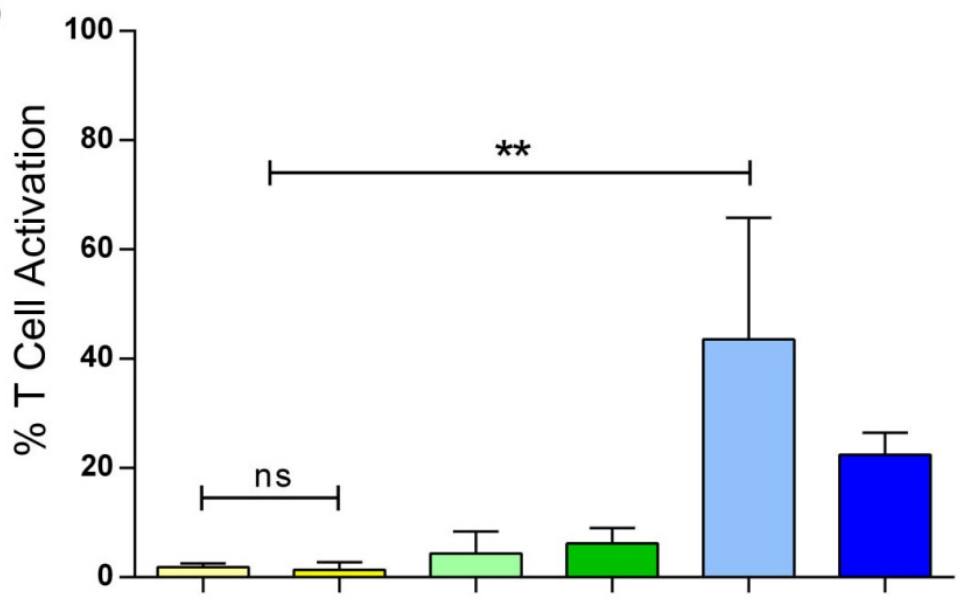

Ratio 1:10

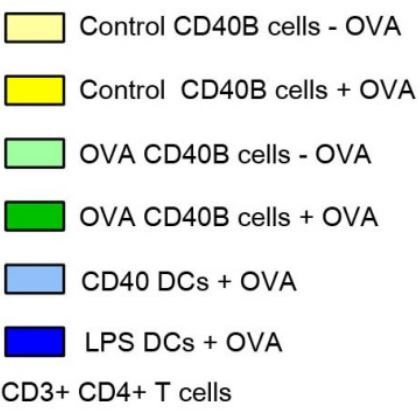

Fig. 3.65 T cell activation in co-cultures of APCs and OT-II or OT-I T cells in the ratio 1:10. Polyclonal CD40B cells (control CD40B cells), OVA-specific CD40B cells (OVA CD40B cells) CD40-matured DCs (CD40 DCs) or LPS-matured DCs (LPS DCs) were left untreated (- OVA) or pulsed with protein (+ OVA) over night. Afterwards, they were mixed with OT-I (A) or OT-II (B) T cells in a ratio of 1:10 and incubated for 5 days. Activation of T cells was determined by staining for CD25 expression and subsequent flow cytometry analysis. Bar charts represent mean values \pm SD of 4 independent experiments. Significant differences were calculated with two-way ANOVA are marked by an asterisk. $* * \mathrm{p} \leq 0.01,{ }^{* * *} \mathrm{p} \leq 0.001, * * * * \mathrm{p} \leq 0.0001$. 
Results

\subsection{Antigen-Specific CD40B Cells Migrate to Secondary Lymphoid Organs and to the Tumor}

For the induction of immunity, APCs need to encounter T cells. This process is particularly regulated by chemokine gradients in the T-cell areas of secondary lymphoid organs (von Andrian and Mempel 2003). By expressing molecules and receptors crucial for homing to secondary lymphoid organs or attraction of T cells (von Bergwelt-Baildon et al. 2006, Guo et al. 2009), they fulfill an important criterion for an efficient APC. However, little is known about the in vivo migration of CD40-activated B cells. To assess their migration kinetics in vivo, CD40B cells were generated from $\mathrm{Luc}^{+} \mathrm{C} 57 \mathrm{BL} / 6$ mice. B cells from $\mathrm{Luc}^{+}$mice could be stimulated with the CD40L without affecting their autofluorescence. Moreover, these cells displayed the typical APC phenotype (Fig. 3.42-3.45). Polyclonal CD40B cells were injected via two different routes and their location was tracked after different time points. Administration routes were chosen to assess the following biological and clinical aspects: first, possible paths of homing to peripheral lymph nodes, lymph or blood vessels; and second, their relevance for clinical application. The general migration of polyclonal CD4OB cells after intravenous (i.v.) or subcutaneous (s.c.) injection was assessed by injecting $5 \times 10^{6}$ Luc $^{+}$CD40B cells (> $90 \%$ pure) into C57BL/6 mice. Subcutaneous injection did not result in migration to the secondary lymphoid organs at any of the observed time points (Fig. 3.66, right column). However, after i.v. injection the $\mathrm{Luc}^{+} \mathrm{CD} 40 \mathrm{~B}$ cells appeared in the spleen within 12 hours and over a period of 5 day migrated to the abdominal lymph nodes (Fig. 3.66, left column). Luc ${ }^{+} \mathrm{CD} 40 \mathrm{~B}$ cells could be detected in the abdominal lymph nodes for up to 15 days (data not shown). 


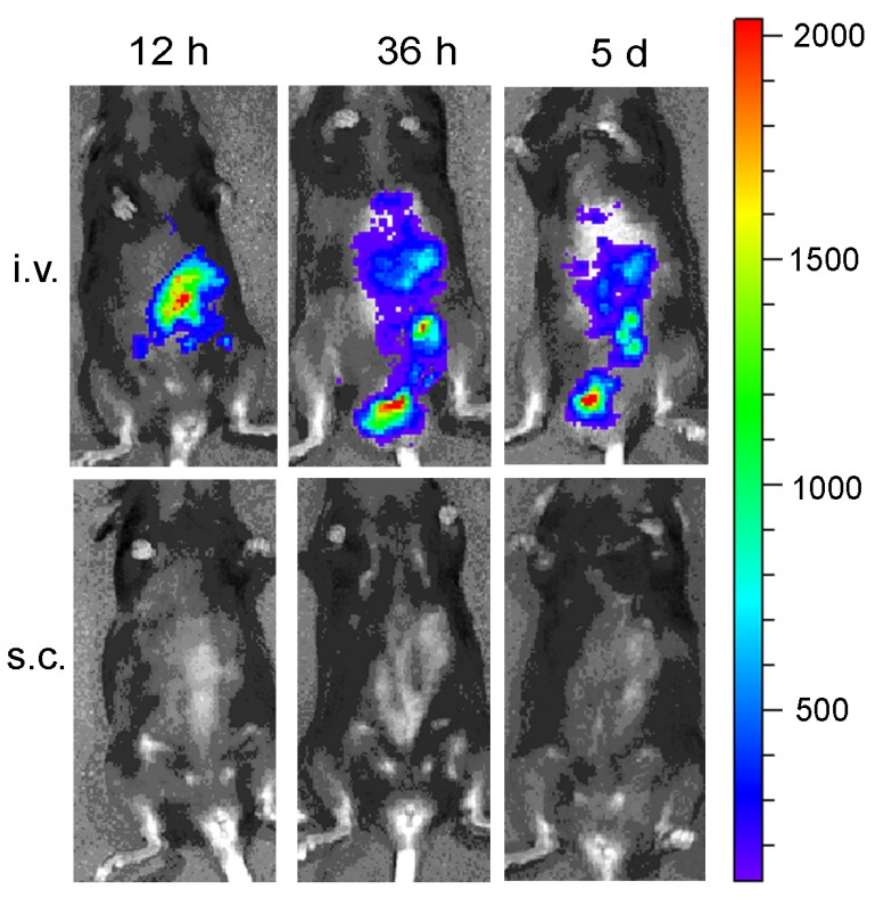

Fig. 3.66 CD40B cells migrate to spleens and lymph nodes in vivo. 5$10 \times 10^{6} \mathrm{CD} 40 \mathrm{~B}$ cells from $\mathrm{Luc}^{+}$mice were injected into $\mathrm{C} 57 \mathrm{BL} / 6$ mice subcutaneously

(s.c.) or intravenously (i.v.). 12 hours (12 h), 36 hours ( $36 \mathrm{~h}$ ) or 5 days ( $5 \mathrm{~d}$ ) after injection, mice were analyzed for the presence of the CD40B cells by imaging in the IVIS 200 system. Representative pictures out of 3 experiments are shown.

To observe the migration behavior of OVA-specific CD40B cells in healthy mice, $1 \times 10^{6}$ Luc $^{+}$ OVA-specific CD4OB cells (> $80 \%$ B cells purity and > $60 \%$ OVA purity) were injected i.v. into C57BL/6 mice. Their migration behavior was similar to that of polyclonal CD40B cells. OVAspecific CD4OB cells appeared in the spleen within 12 hours and over a period of 5 days migrated to the abdominal lymph nodes (Fig. 3.67). They could be detected for up to 15 days (data not shown).

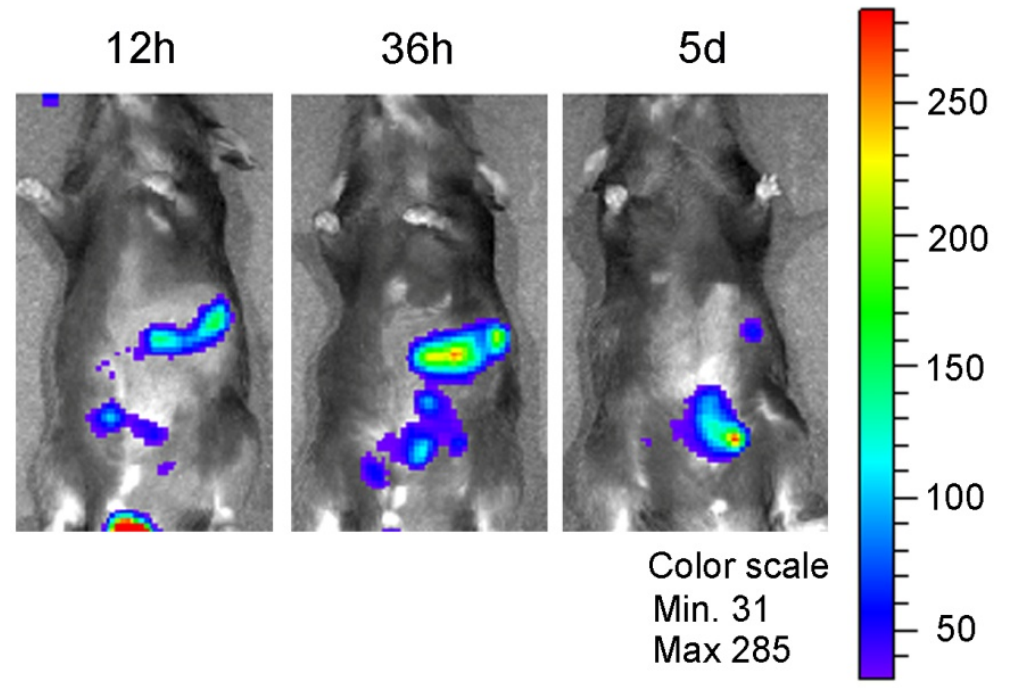

Fig. 3.67 OVA-specific CD40B cells migrate to spleens and lymph nodes in vivo. $1 \times 10^{6}$ CD40B cells from Luc ${ }^{+}$mice were injected into C57BL/6 mice intravenously. 12 hours (12 h), 36 hours $(36 \mathrm{~h})$ or 5 days $(5 \mathrm{~d})$ after injection, mice were analyzed for the presence of the CD40B cells by imaging in the IVIS 200 system. Representative pictures out of 3 experiments are shown.

Tumor-infiltrating B cells in human breast cancer (Hansen et al. 2001) and B cells in the tumor-draining lymph nodes in mice (Li et al. 2009) were shown to produce autoantibodies against tumor targets, thereby suggestion an infiltration of tumors by antigen-specific B 
cells. In order to test this hypothesis, the migration behavior of polyclonal and OVA-specific CD40B cells was assessed in tumor-bearing mice. For this purpose, OVA-expressing E.G7 lymphoma cells were injected s.c. into the right flanks of C57BL/6 mice and tumors were allowed to grow for 10 days until they became clearly apparent. EG.7 lymphoma cells were analyzed for their expression of OVA-protein in the context of MHC II by FACS (Fig. 3.68).

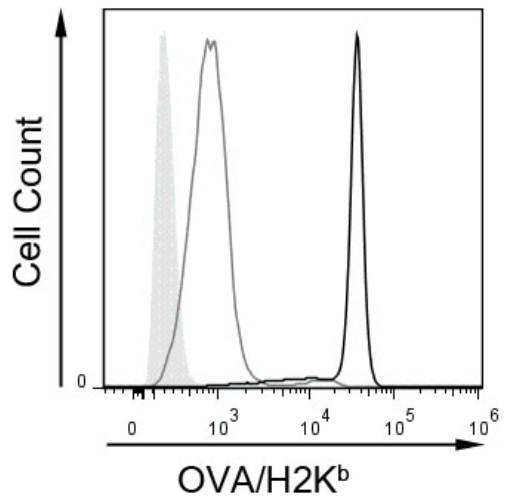

Fig. 3.68 Expression of OVA-protein in the context of MHC II on E.G7 lymphoma cells. Representative flow cytometry analyses of E.G7 lymphoma cells out of at least 5 independent experiments are shown. Cells were stained for the expression of OVA-peptide in the context of MHC II (black line). EL4 cells served as negative controls (grey line). Unstained cells served as staining control (filled line).

$1 \times 10^{6}$ Luc $^{+}$OVA-specific or polyclonal CD40B cells were injected i.v. into tumor-bearing mice and their location was tracked for 14 days. Polyclonal CD40B cells showed the same migration behavior as in healthy mice by traveling to the spleen and the lymph nodes and in addition to the tumor draining lymph nodes. No Luc ${ }^{+}$cells showed up in the tumor (Fig. 3.69).

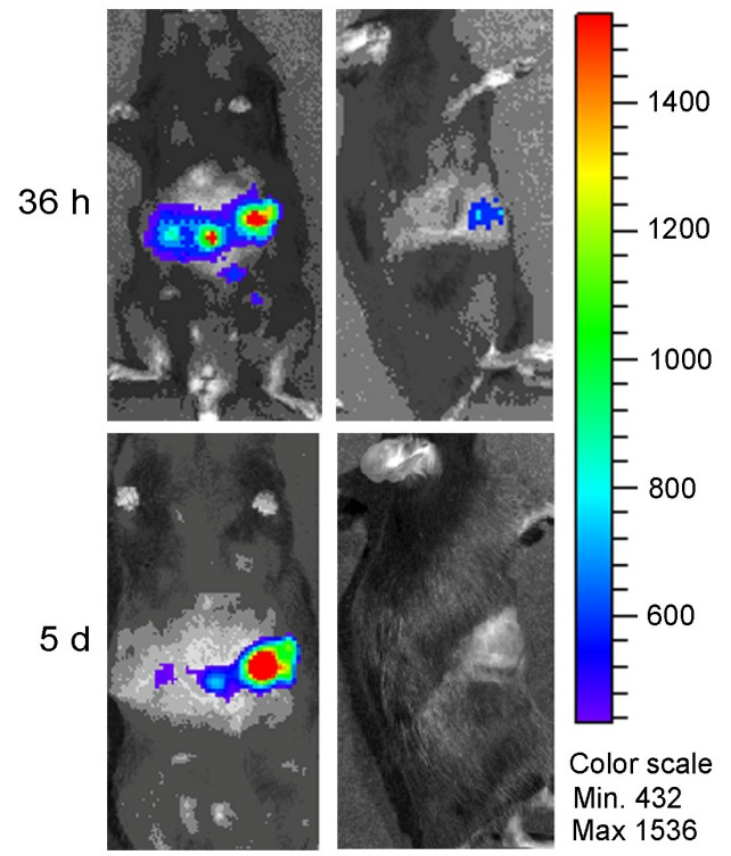

Fig. 3.69 Polyclonal CD40B cells migrate to lymphoid organs in tumor-bearing mice. $1 \times 10^{6}$ CD40B cells from Luc $^{+}$mice were injected into C57BL/ 6 mice intravenously. 36 hours ( 36 h) or 5 days ( $5 \mathrm{~d}$ ) after injection, mice were analyzed for the presence of the CD40B cells by imaging in the IVIS 200 system. Representative pictures out of 3 experiments are shown. 
OVA-specific CD40B cells migrated to the spleen and the lymph node over a period of 36 hours (Fig. 3.70). However, in contrast to polyclonal CD40B cells, they also appeared at the tumor site in high amounts where they still could be detected on day 5 . Some Luc ${ }^{+}$cells stayed in the spleen and the abdominal lymph nodes.

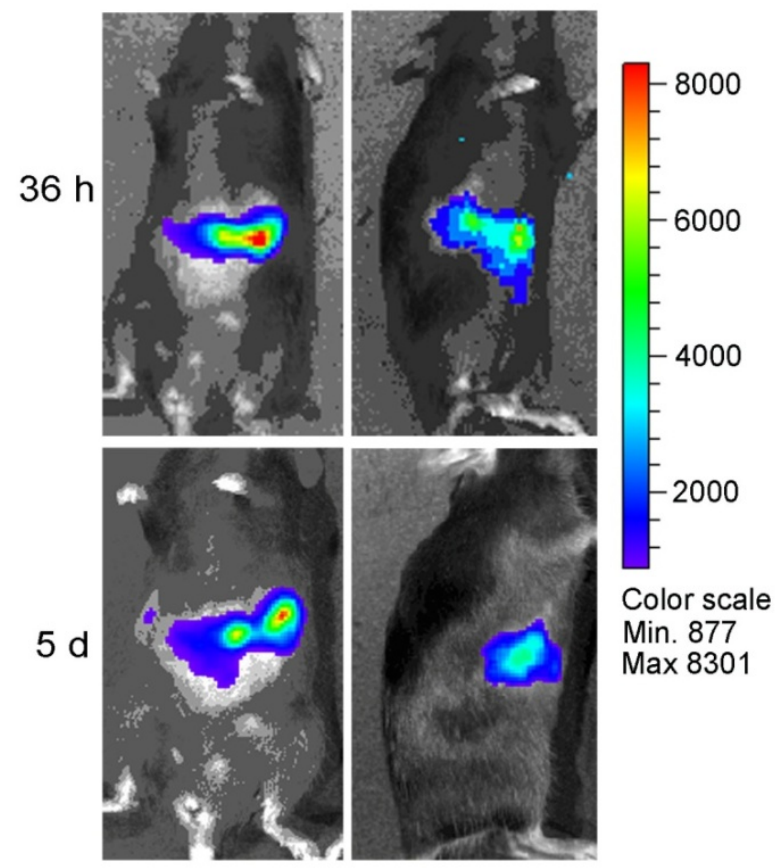

Fig. 3.70 CD40B cells migrate to lymphoid organs and the tumor in tumor-bearing mice. $1 \times 10^{6} \mathrm{CD} 40 \mathrm{~B}$ cells from $\mathrm{Luc}^{+}$mice were injected into C57BL/6 mice intravenously. 36 hours $(36 \mathrm{~h})$ or 5 days $(5 \mathrm{~d})$ after injection, mice were analyzed for the presence of the CD40B cells by imaging in the IVIS 200 system. Representative pictures out of 3 experiments are shown.

\subsection{Antigen-Specific CD40B Cells Induce an Antigen-Specific Immune}

\section{Response In Vivo}

When used as cellular adjuvant, polyclonal CD40B cells are able to present antigen to $T$ cells in order to induce an antigen-specific immune response in vivo (Wennhold et al. 2013) and unpublished data). Moreover, OVA-specific CD4OB cells migrate to the spleen and lymph nodes when administered i.v. Based on the previous in vitro data, antigen-specific CD40B cells should be more effective in inducing an antigen-specific immune response in vivo than polyclonal CD40B cells. In order to test this hypothesis, in vivo cytotoxicity assays were performed. For this purpose, different APCs were loaded with protein by protein-pulsing, which is a common technique to deliver antigens to APCs (Inaba et al. 1990, Zitvogel et al. 1996). Protein pulsed APCs were injected according to a previously optimized vaccination protocol (unpublished data) (Fig. 3.71): C57BL/6N mice were immunized i.v. three times in 
an interval of seven days with $5 \times 10^{6}$ APCs. On day $21, \mathrm{CFSE}^{+}$OVA-peptide pulsed target cells were injected into immunized mice.

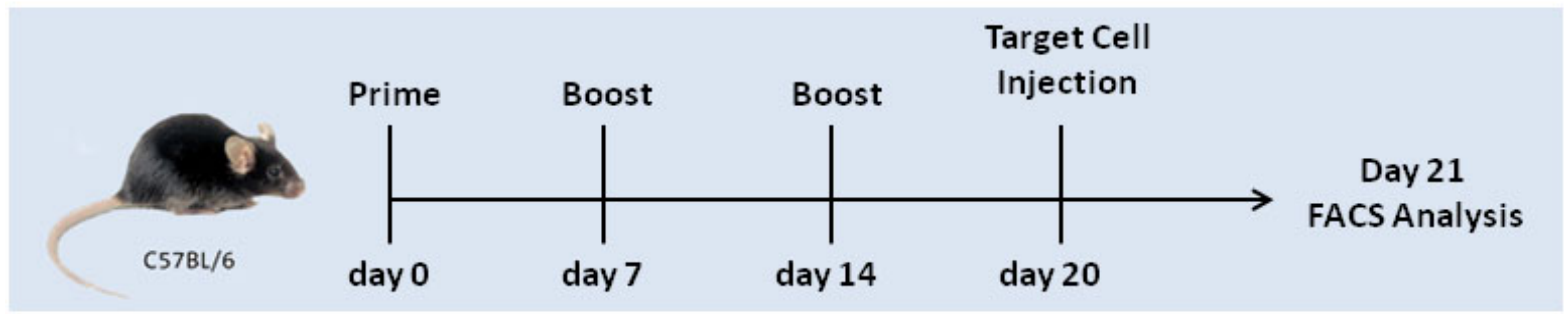

Fig. 3.71 Overview of the in vivo cytotoxicity assay. Different APCs loaded with protein were injected three times in an interval of seven days. Peptide-pulsed CFSE-labeled target cells were injected on day 21 . On day 22 , specific lysis of the target cells was analyses by flow cytometry.

The in vivo cytolytic activity of antigen-specific $\mathrm{CD}^{+} \mathrm{T}$ cells was determined by calculating the specific lysis from the ratio of target cells in spleens. The specific lysis of immunized mice was normalized to the specific lysis of negative controls.

Polyclonal and OVA-specific CD4OB cells were generated from non-immunized or OVAimmunized mice, respectively, by $\mathrm{CD} 19^{+}$selection and subsequent $\mathrm{CD} 40$-activation. Proteinpulsed DCs served as standard to which CD4OB cells were compared. Since isolated $C D 19^{+} B$ cells of OVA-immunized mice consisted of 1-4 \% OVA-specific B cells, DCs were either injected at $93 \%$ purity as a control (Fig. 3.54) or they were supplemented with $96-99 \%$ polyclonal CD40B cells (DCs+CD40Bs) to mimic OVA-specific B cell purity. All APCs showed upregulation of activation markers before injection (Fig. 3.42-3.45+3.55). Positive controls were immunized with OVA-protein and CpG in PBS and IFA. Negative controls were treated with IFA and PBS only.

The antigen-specific response was significantly higher in mice that were immunized with OVA-specific CD40B cells $(27.51 \% \pm 14.57)$ than in mice that were immunized with polyclonal control CD40B cells (11.12 \% \pm 4.92$)$ (Fig. 3.72). Immunization with OVA-specific CD40B cells, pure DCs, and positive controls resulted in similarly effective induction of specific lysis. However, induction of immunity was significantly higher in mice immunized with OVA-specific CD40B cells than in mice immunized with DCs+CD40Bs $(8.17 \% \pm 3.12)$. 


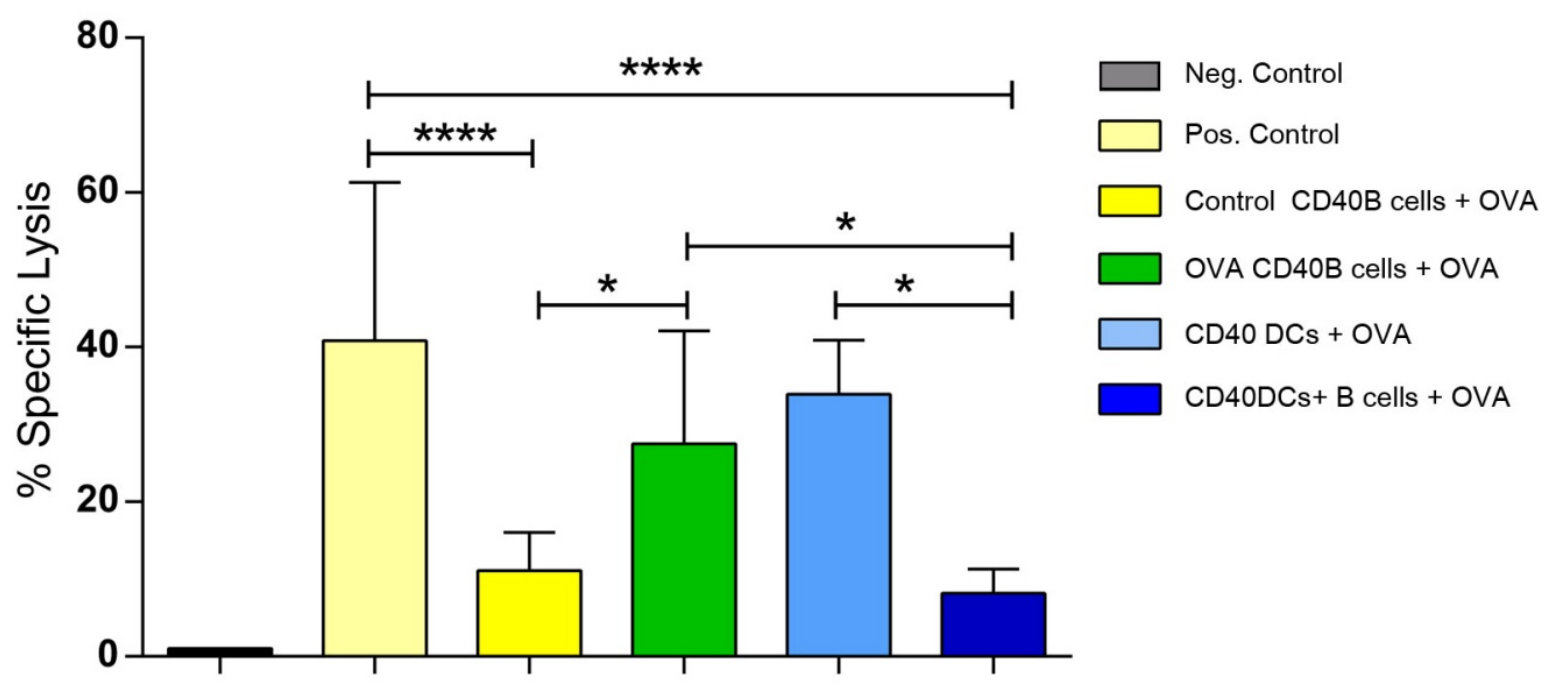

Fig. 3.72 Antigen-specific CD40B cells induce specific lysis of target cells. C57BL/6N mice were immunized with $5 \times 10^{6} \mathrm{APCs}$, i.e. polyclonal CD40B cells (Control CD40Bs), OVA-specific CD40B cells (OVA CD40Bs), pure dendritic cells (CD40 DCs) or DCs + polyclonal CD40B cells (CD40DCs+B cells), PBS+IFA as negative controls (Neg. Control), or IFA+PBS+OVA+CpG as positive controls (Pos. Control) three times i.v. in an interval of seven days. The ratio of $\mathrm{CFSE}^{+}$target cells in spleens was determined by FACS (A) and the specific lysis was calculated by normalizing values to negative controls (B). Representative FACS data of at least three independent experiments are shown (A). Bar charts show mean values $\pm S D$ of specific lysis of three independent experiments with three mice per group (B). Significant differences calculated with ordinary one-way ANOVA are marked by an asterisk. $* \mathrm{p} \leq$ $0.05, * * * * p \leq 0.0001$.

\subsection{Antigen-Specific B Cells Differentiate into Antibody-Secreting B Cells upon}

\section{Stimulation}

The classic effector function of B cells is not the presentation of antigens to T cells, but the secretion of antibodies upon differentiation into plasma cells. Although several monoclonal antibodies are approved for the treatment of cancer in humans, they have several drawbacks, e.g. their development is expensive and laborious (Chames et al. 2009, Weiner et al. 2010) and even humanized monoclonal antibodies can be antigenic (Klee 2000). Therefore, it seems plausible to transfer patient-derived plasma cells that produce tumorantigen-specific antibodies (Moutai et al. 2014). Moreover, the combination of both effector functions offers additional advantages for cancer immunotherapy.

Therefore, a protocol was developed to stimulate differentiation of antigen-specific B cells into antibody secreting plasma cells using the well known stimulator of plasma cell differentiation, IL-21 (Ozaki et al. 2004, Moutai et al. 2014), in combination with other 
stimulating cytokines and BCR antagonists. For this purpose, OVA-specific B cells were isolated from immunized C57BL/6 mice ( $80 \%$ B cell purity and $>60 \%$ OVA purity) and were incubated with the stimuli for 3 days. Plasma cell differentiation was determined by FACS analyses for the percentage of $\mathrm{CD}_{138}{ }^{+} \mathrm{B}$ cells. Polyclonal B cells from non-immunized mice served as control.

The addition of IL-21 to OVA-specific B cell cultures increased the percentage of B220 $\mathrm{CD} 9^{+} \mathrm{CD} 138^{+}$plasma cells from $13.28 \% \pm 7.70$ to $18.27 \% \pm 6.94$ (Fig. 3.73, left panel). In addition of soluble CD40L the percentage of $\mathrm{CD} 138^{+}$plasma cells significantly increased to $44.24 \% \pm 0.76$. However, IL-21 was crucial for this effect, since stimulation of OVA-specific $B$ cells with OVA-antigen, IL-4 and CD4OL alone resulted in reduced percentage of $\mathrm{CD} 138^{+} \mathrm{B}$ cells $(16.89 \% \pm 13.55)$. OVA-specific B cells that were treated with IgM and IL-4 alone were used as negative controls $(12.22 \% \pm 7.22)$ and OVA-specific B cells that were treated with LPS served as positive controls (58.09 \% \pm 23.28$)$. LPS and IgM treated cells were not additionally treated with OVA-antigen. However, the amount of OVA-antigen added during the purification process is sufficient to results in stimulation as indicated by MLRs of 'unpulsed' OVA-specific B cells (chapter 3.3).

Control B cells from non-immunized mice, which showed a different phenotype upon isolation, reacted differently to the stimuli than OVA-specific B cells (Fig. 3.73, right panel). Treatment with OVA-antigen and IL-4 alone or in combination with IL-21 and treatment with IgM and IL-4 almost similarly induced plasma cell differentiation $(23.63 \% \pm 16.10,22.69 \% \pm$ 14.44 and $25.42 \% \pm 13.70$, respectively). In comparison, treatment with CD4OL reduced the differentiation into CD138 ${ }^{+}$plasma cells $(15.71 \% \pm 2.56$ with OVA+ IL-4+ IL-21+ CD40L and $10.79 \% \pm$ with OVA+ IL-4+ CD4OL). However, stimulation with LPS resulted in the highest percentages of $\mathrm{CD} 138^{+} \mathrm{B}$ cells $(38.99 \% \pm 20.59)$. 


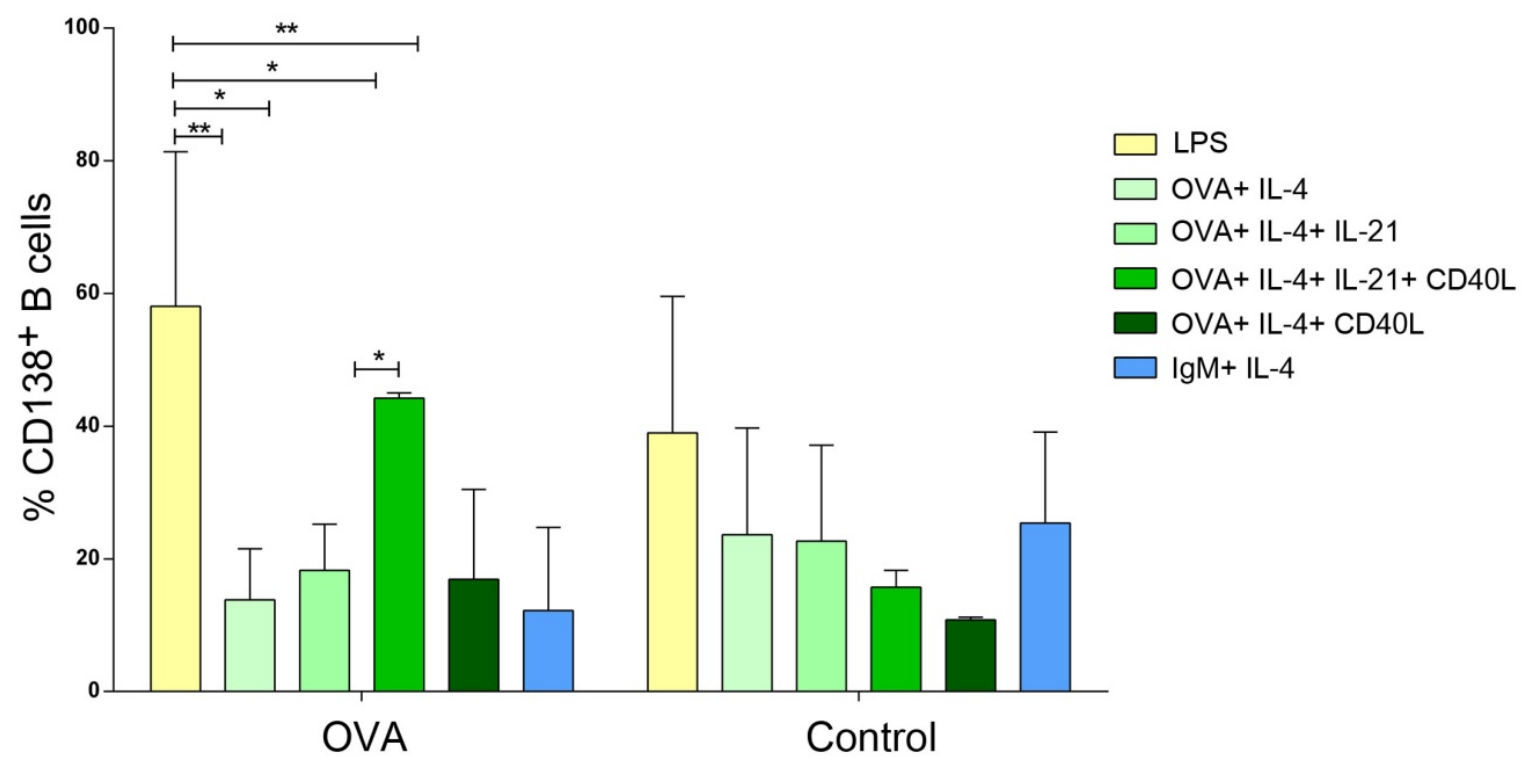

Fig. 3.73 Induction of plasma cell differentiation in antigen-specific and polyclonal $B$ cells. OVAspecific $B$ cells (OVA) of immunized mice or $\mathrm{CD}^{1} 9^{+} \mathrm{B}$ cells of non-immunized mice (Control) were stimulated with the indicated stimuli for 3 days and thereafter analyzed by flow cytometry for expression of CD138. Bar charts show mean values \pm SD of the percentage of $C D 138^{+} B$ cells in three independent experiments. Significant differences calculated with two-way ANOVA are marked by an asterisk. ${ }^{*} p \leq 0.05,{ }^{* *} p \leq 0.01$.

Another possibility to enrich antigen-specific B cells is a memory isolation kit, which works by specifically isolating $\operatorname{lgG}^{+}$B cells. In order to confirm that stimulation with the specific antigen is necessary for the differentiation into plasma cells from antigen-specific B cells, KLH-specific B cells were purified by the memory isolation kit and stimulated with the same stimuli as shown for OVA-specific B cells. However, instead of stimulating KLH-specific B cells with their specific antigen, the non-specific OVA-protein was added. Unexpectedly, even though the specific antigen KLH was not present, this experiment showed similar results as OVA-specific B cells (Fig. 3.74 compared to Fig. 3.73). KLH-specific B cells seem to be stimulated by anti-lgG antibodies contained in the memory isolation kit and therefore respond by differentiation into plasma cells when stimulated with additional cytokines. In OVA-specific B cells, purification with the memory isolation kit and additional treatment with OVA-protein does not result in a higher differentiation into plasma cells than with OVAprotein alone (data not shown). The lack of a method for the isolation of specific B cells without stimulating the $B C R$, makes it difficult to determine the role of $B C R$ signaling in plasma cell differentiation. 


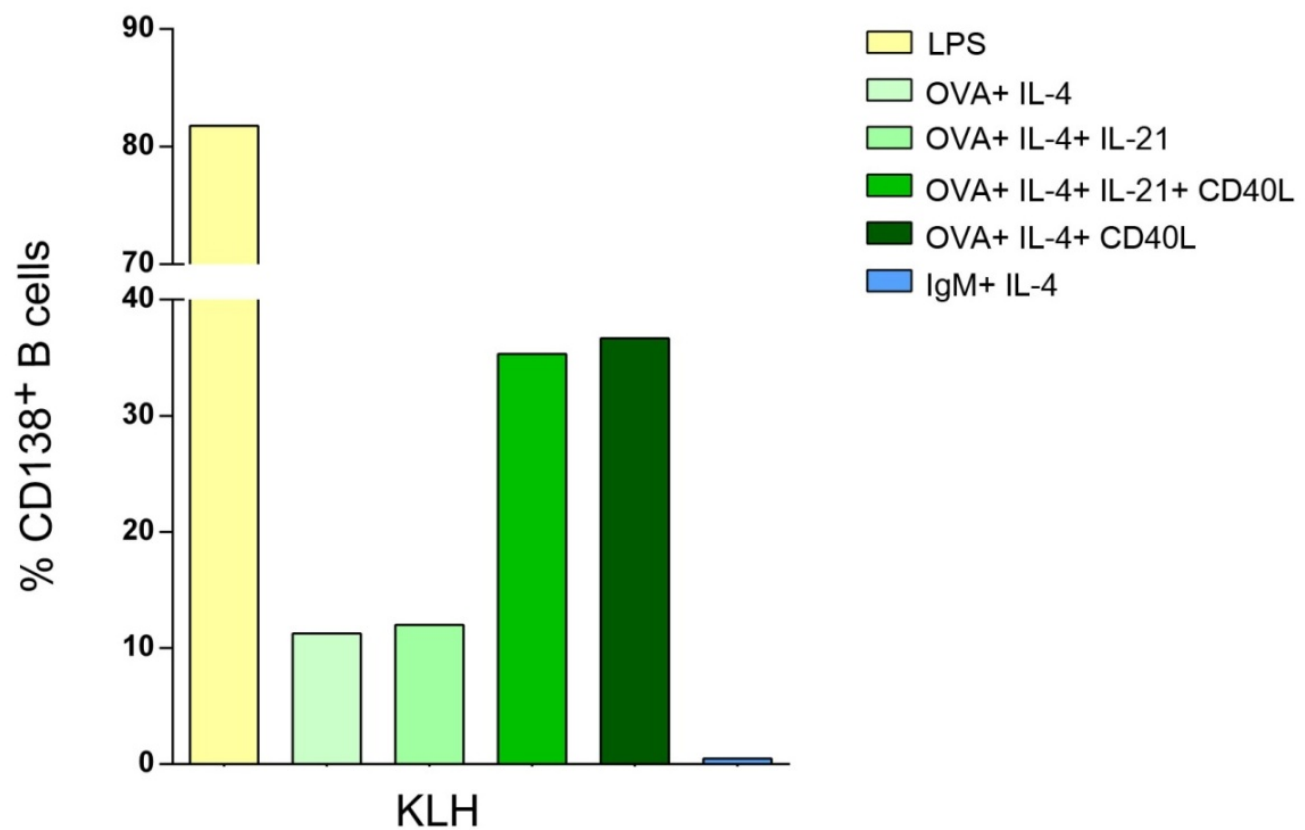

Fig. 3.74 Induction of plasma cell differentiation in antigen-specific B cells. KLH-specific B cells (KLH) of immunized mice were stimulated with the indicated stimuli for 3 days and thereafter analyzed by flow cytometry for expression of CD138. Bar charts show values of the percentage of CD138 ${ }^{+} \mathrm{B}$ cells of one representative experiment.

Clearly the most important aspect when thinking about OVA-specific plasma cells for immunotherapy is the actual secretion of OVA-specific antibodies. Therefore, the supernatant of the differentiation cultures were collected and an OVA-specific Elisa was performed. As expected, control B cells and KLH-specific B cells did not secrete any OVAspecific IgG1 antibodies when treated with OVA-protein or any of the other stimuli (Fig. 3.75). In accordance with the observed changes in $\mathrm{CD}_{138^{+}} \mathrm{B}$ cells, OVA-specific B cells secreted OVA-specific IgG1 antibodies when treated with OVA-protein and IL-4 $(4.53 \mathrm{ng} / \mathrm{ml})$, OVA-protein + IL-4 + IL-21 (5.72 ng/ml) or OVA-protein + IL-4 + IL-21 + CD40L (6.44 ng/ml). Moreover, stimulation with OVA-protein, IL-4 and CD40L without IL-21 led to a reduced secretion of OVA-specific IgG1 antibodies $(3.64 \mathrm{ng} / \mathrm{ml})$. As expected, OVA-specific B cells produced higher amounts of OVA-specific IgG1 antibodies when treated with LPS (25.52 $\mathrm{ng} / \mathrm{ml})$. In contrast to the low percentage of $\mathrm{CD} 138^{+} \mathrm{B}$ cells in OVA-specific $\mathrm{B}$ cells that were treated with IgM and IL-4, the secretion of OVA-specific IgG1 antibodies was relatively high $(11.65 \mathrm{ng} / \mathrm{ml})$. 


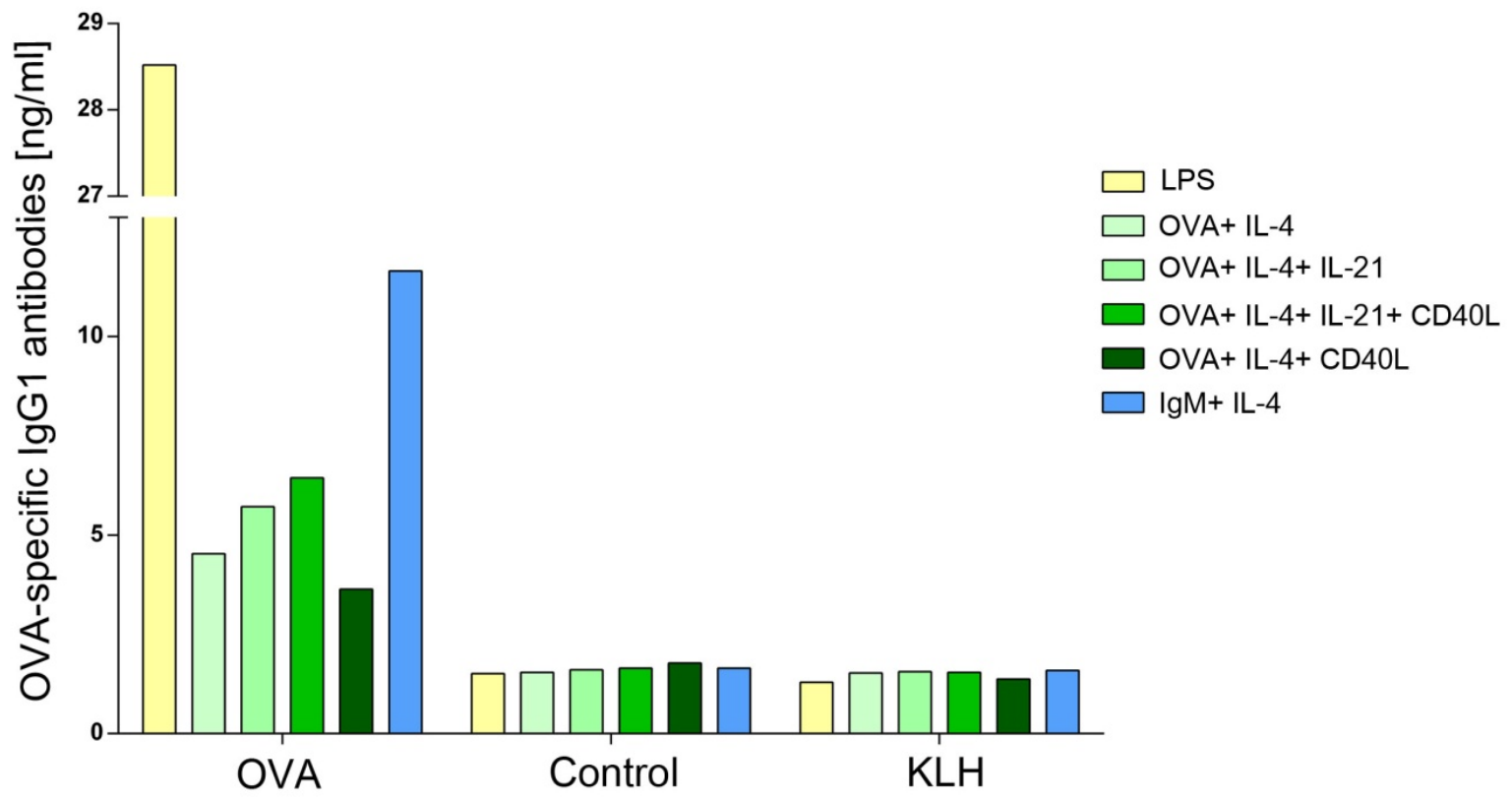

Fig. 3.75 Secretion of OVA-specific IgG1 antibodies in stimulated culture supernatants. OVA-specific $B$ cells (OVA) or KLH-specific B cells (KLH) of immunized mice or $C D 19^{+} B$ cells of non-immunized mice (Control) were stimulated with the indicated stimuli for 3 days and supernatants were analyzed by ELISA for the production of OVA-specific IgG1 antibodies. Bar charts show mean values \pm SD of antibody concentration in $\mathrm{ng} / \mathrm{ml}$ of one independent experiment.

For in vivo experiments, OVA-specific B cells were stimulated with the combination of OVAprotein, IL-4, IL-21 and CD40L, since differentiation into CD138 ${ }^{+}$cells and secretion of specific antibodies were high and cells survived best over the culture period of 3 days (data not shown).

\subsection{The Combined Vaccination with Antigen-Specific CD40B Cells and Plasma}

\section{Cells Induces Anti-Tumor Immunity and Prolongs Survival}

The experiments so far have confirmed the hypothesis that OVA-specific CD4OB cells more efficiently induce an antigen-specific T cell response than polyclonal CD4OB cells in vitro and in vivo. Therefore, the influence of a preventive immunization with tumor-antigen specific CD40B cells on tumor establishment and growth was investigated. The E.G7 lymphoma cells express OVA-protein in the context of $\mathrm{MHCI}$ and gives rise to $\mathrm{H}-2 \mathrm{~Kb}$ restricted cytotoxic lymphocytes specific for the OVA 258-276 peptide and was therefore chosen as tumor model. For preventive immunization, protein-pulsed APCs were injected into C57BL/6N mice i.v. three times in an interval of seven days with APCs numbers varying between 0.1 and 0.6 $x 10^{6}$ (Fig. 3.76). On day 21, immunized mice were inoculated with E.G7 tumor cells, which 
were analyzed for their expression of OVA-protein in the context of MHC I by FACS (Fig. 3.68). Tumor growth was observed the earliest on day 7 after tumor inoculation and daily measured from there on.

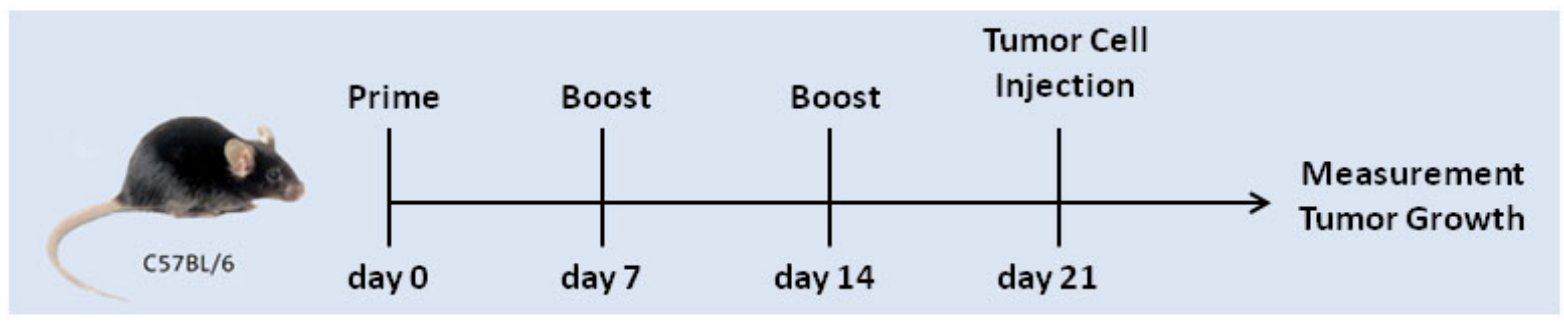

Fig. 3.76 Overview of the tumor vaccination scheme. Different APCS loaded with protein were injected three times in an interval of seven days. EG.7 lymphoma cells were injected on day 21. Tumor growth was measured thereafter.

Polyclonal $\mathrm{CD} 40 \mathrm{~B}$ cells were isolated from non-immunized mice by $\mathrm{CD}_{19^{+}}$selection. OVAspecific CD40B cells were isolated from immunized mice and purified by positive selection. Both B cell populations were activated by CD40 stimulation for 2 days. On the day of injection, polyclonal B cell cultures consisted of $>95 \% \mathrm{CD} 19^{+} \mathrm{B}$ cells and OVA-specific B cell cultures consisted of $>80 \% \mathrm{CD} 19^{+}>60 \%$ OVA-specific B cells. Protein-pulsed DCs served as standard, to which CD4OB cells were compared, and DCs were injected at $93 \%$ purity (Fig. 3.54). The APC phenotype of B cells and DCs was confirmed by FACS analyses before injection (Fig. 3.42-3.45+3.55). 0.1-0.6 × $10^{6}$ cells were injected in $100 \mu \mathrm{l}$ PBS. Positive controls were immunized with OVA-protein and CpG in IFA. Negative controls were treated with IFA and PBS only. For plasma cell differentiation, OVA-specific B cells were stimulated with the combination of OVA-protein, IL-4, IL-21 and CD40L. Secretion of OVA-specific IgG1 antibodies was confirmed by ELISA (Fig. 3.77).

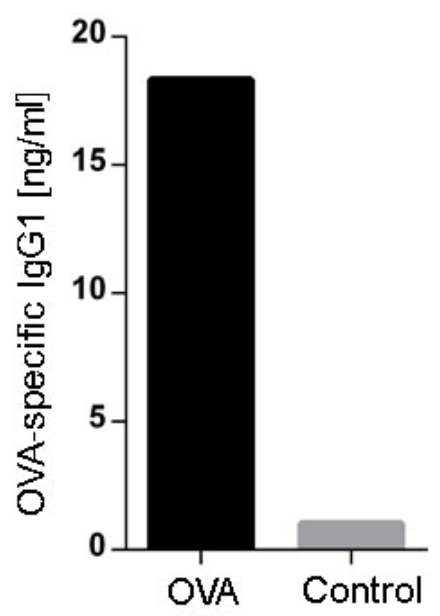

Fig. 3.77 Secretion of OVA-specific IgG1 antibodies. OVAspecific B cells were stimulated with OVA-protein, IL-4, IL-21 and $\mathrm{CD} 40 \mathrm{~L}$ for 3 days in order to stimulate plasma cell differentiation. The supernatant was collected and the concentration of OVA-specific IgG1 antibodies was determined by ELISA. Polyclonal $\mathrm{CD} 19^{+} \mathrm{B}$ cells served as control. Bar charts represent one representative experiment. 
Mice that were immunized with APCs before tumor inoculation showed a delayed tumor growth compared to negative controls (IFA + PBS only) and positive controls (IFA + CpG+ OVA-Protein + PBS) (Fig. 3.78). On day 16 after tumor inoculation, the difference in tumor volume of negative controls was significant to all other tested groups. Mice of the negative control group died much earlier than the mice in all other groups and this difference was significant to mice treated with $\mathrm{CD} 40 \mathrm{~B}$ cells alone or in combination with plasma cells (Fig. 3.79). Although tumors of positive controls grew faster and the mice died earlier than mice that were treated with APCs, the differences were not significant.

Comparing the different APC treated groups, CD4OB cells prolonged survival more efficiently than did DCs. Tumors of mice that were treated with control CD40Bs grew faster than tumors of mice treated with DCs. Nevertheless, mice of the control CD40B group died later than mice that were treated with DCs. Both differences were not significant. Tumors of mice that were treated with control CD40Bs grew also faster than tumors of mice that were treated with OVA CD4OBs alone or combination with plasma cells. This difference was significant on day 18. Moreover, mice treated with OVA CD40Bs alone or in combination with plasma cells survived longer than control CD40B treated mice.

Tumors in DC treated mice grew faster and the mice died earlier than tumors of mice treated with OVA CD40Bs alone or in combination with plasma cells. The difference was not significant on day 24 to OVA CD40Bs alone, but to OVA CD4OBs in combination with plasma cells.

The addition of plasma cells also resulted in a significant difference in tumor growth between the mice treated with OVA CD4OBs and mice treated with CD40Bs in combination with plasma cells.

The difference in survival of mice that were treated with OVA CD40Bs in combination with plasma cells was significant to all other groups except for mice treated with OVA CD4OBs alone or plasma cell alone.

Tumors of mice that were treated with plasma cells alone grew slower than tumors of negative controls for the first 13 days, but afterwards in two of three mice the tumor volume increased rapidly. However, one mouse stayed tumor free until the end of the experiment and this difference was significant to negative controls. 


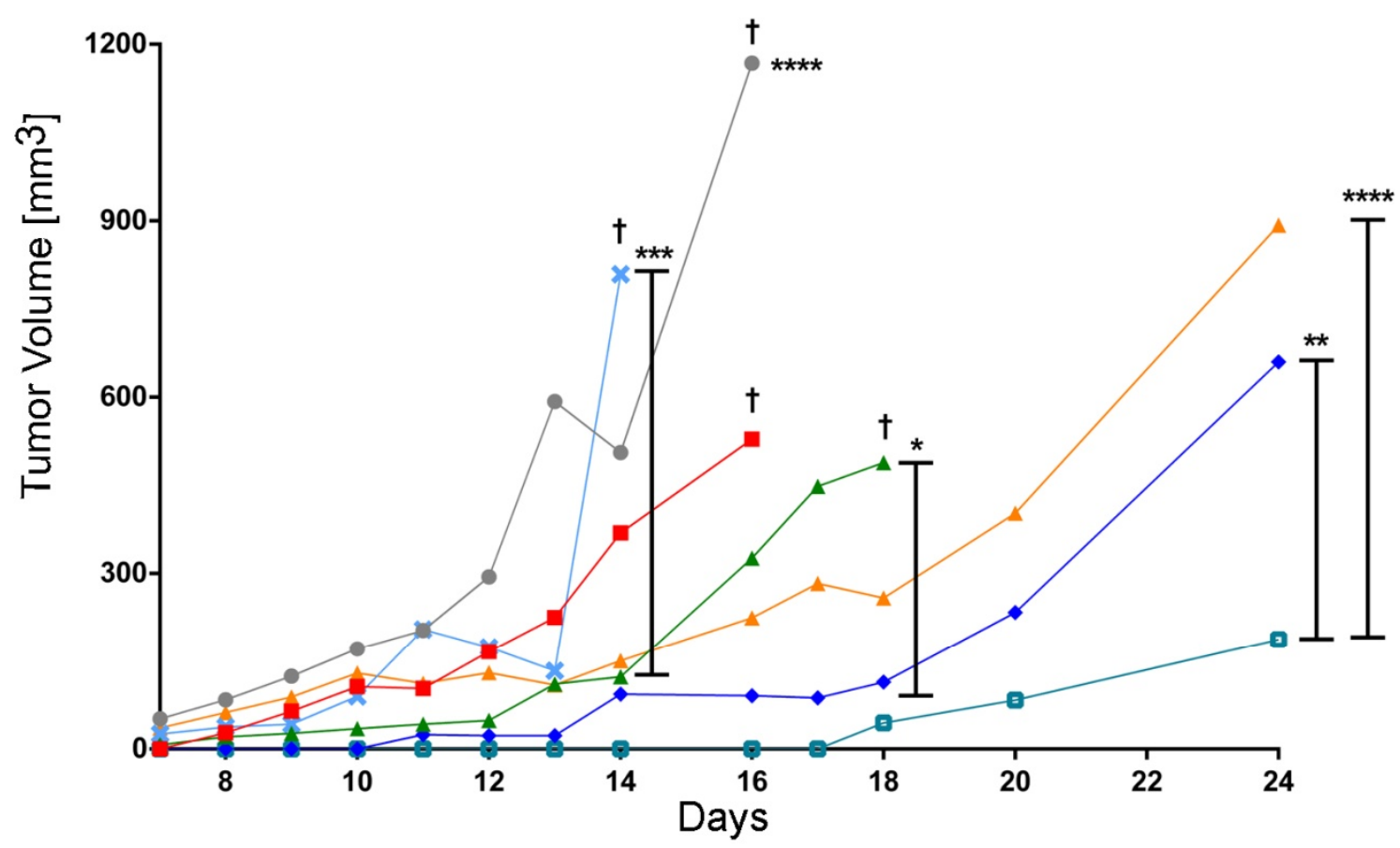

Fig. 3.78 Antigen-specific CD40B cells and plasma cells induce anti-tumor immunity. C57BL/6N mice were immunized with 0.1-0.6 $\times 10^{6} \mathrm{APCs}$, i.e. polyclonal CD40B cells (Control CD40Bs), OVA-specific CD40B cells (OVA CD40Bs), pure CD40-activated dendritic cells (CD40 DCs); PBS+IFA as negative controls (NC), IFA+OVA+CpG as positive controls (PC); OVA-specific plasma cells alone (OVA PCs) or in combination with OVA-specific CD40B cells (OVA CD40Bs+ OVA PCs) three times i.v. in an interval of seven days. On day $21,0.4 \times 10^{6}$ E.G7 lymphoma

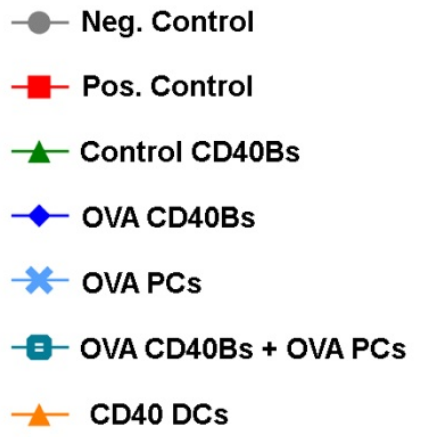
cells were injected into immunized mice and tumor growth was measured every day thereafter. Growth curves show increase of tumor volume in $\mathrm{mm}^{3}$ of one out of three representative experiments with three mice per group. Significant differences calculated with two-way ANOVA are marked by an asterisk. ${ }^{*} p \leq 0.05,{ }^{* *} p \leq 0.01,{ }^{* * *} p \leq$ $0.001, * * * * p \leq 0.0001$. Curves had to be terminated when one mouse of the group had to be sacrificed due to extensive tumor growth as indicated by $\boldsymbol{\dagger}$. 


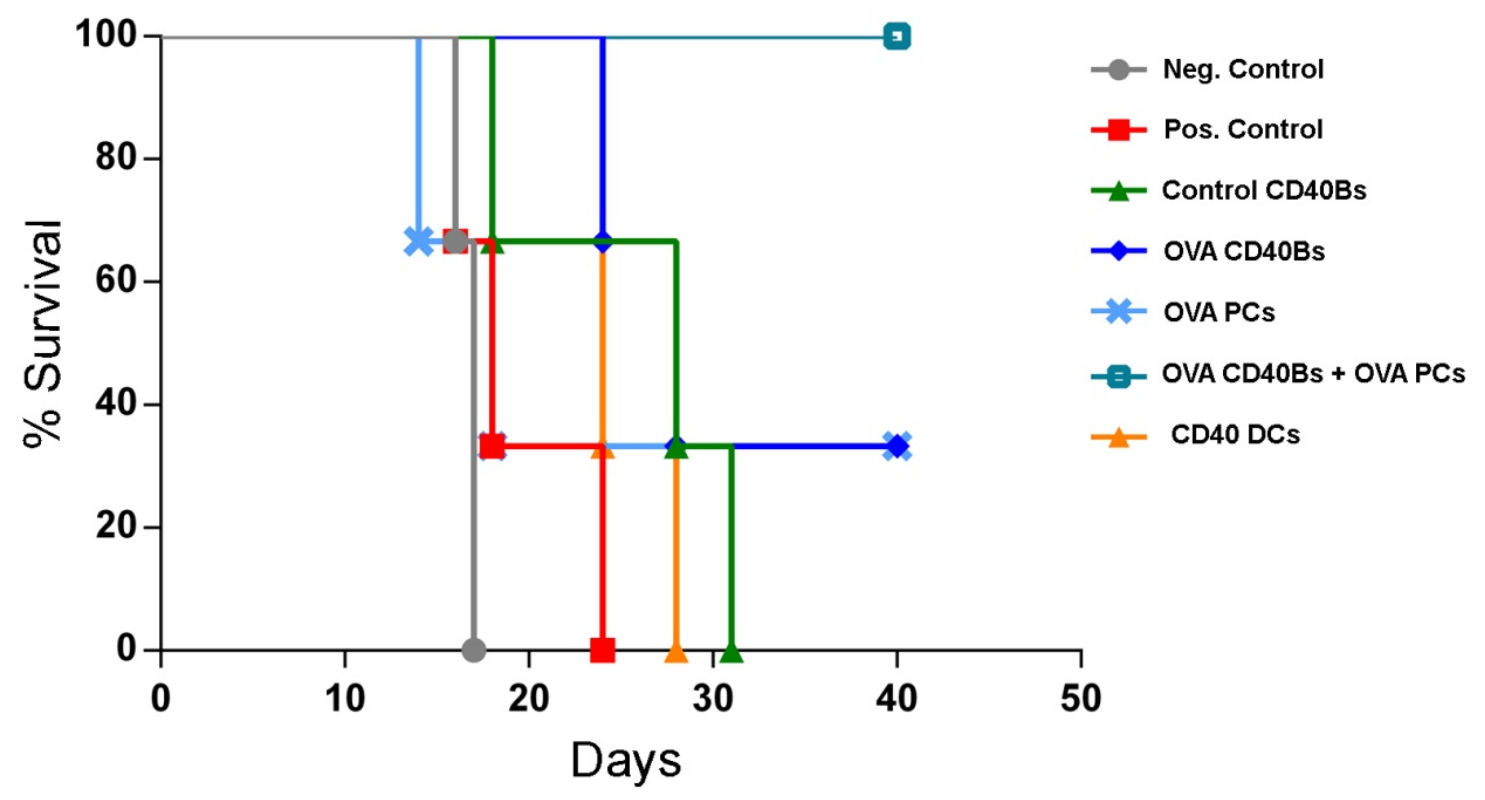

Fig. 3.79 Antigen-specific CD40B cells and plasma cells prolong survival of tumor-bearing mice. C57BL/6N mice were immunized with 0.1-0.6 $\times 10^{6} \mathrm{APCs}$, i.e. polyclonal CD40B cells (Control CD40Bs), OVA-specific CD40B cells (OVA CD40Bs), pure CD40-activated dendritic cells (CD40 DCs); PBS+IFA as negative controls (NC), IFA+OVA+CpG as positive controls (PC); OVA-specific plasma cells alone (OVA PCs) or in combination with OVA-specific CD40B cells (OVA CD40Bs+ OVA PCs) three times i.v. in an interval of seven days. On day 21, $0.4 \times 10^{6} \mathrm{E} . \mathrm{G} 7$ lymphoma cells were injected into immunized mice. Survival of mice was observed over a period of 40 days. Survival curves show one out of three representative experiments with three mice per group. Significant differences were as follows: * OVA PCs vs. Neg. Controls; * OVA CD40Bs vs. Neg. Controls; * Control CD40Bs vs. Neg. Controls; * OVA CD40Bs+ OVA PCs vs. Neg. Controls/ Pos. Controls/ Control CD40Bs/ CD40 DCs. Significant differences calculated with the Mantel-Cox test: ${ }^{*} p \leq 0.05$. 
Discussion 



\section{Discussion}

The present study strongly underlines the potential of CD40B cells as APCs for cancer immunotherapy and adds further advantages by combining induction of T cell immunity with the secretion of specific antibodies, the B cell's most prominent function in immune response.

\subsection{Murine Antigen-Specific B Cells are Class-Switched and Develop an APC Phenotype when Stimulated with the CD40L}

Several studies reported the detection of antigen-specific B cells in immunized mice (Julius et al. 1976, Hayakawa et al. 1987, Hoven et al. 1989, Lalor et al. 1992, Townsend et al. 2001, Newman et al. 2003), but only few focused on the isolation of those cells and some rather relied on the use of mouse models with genetically modified B cells (Phan et al. 2003, Avalos et al. 2014). None of the groups studied the antigen-presenting function of the antigenspecific $B$ cells.

In this study, antigen-specific B cells were isolated from mice and successfully enriched by the use of antigen tetramers. Enrichment highly depended on the grade of biotinylation of the antigen and the efficiency of immunization. This is in accordance with other studies showing that a tetrameric antigen-labeling is crucial to include the whole antigen-specific $B$ cell repertoire (Franz et al. 2011). Detection of antigen-specific B cells in the mesenteric lymph nodes varied, since they do not represent the only draining lymph nodes after i.p. injection (Parungo et al. 2007). Therefore, lymph nodes were not included in the isolation of antigen-specific $B$ cells.

Compared to polyclonal B cells of non-immunized mice and immunized-mice, the antigenspecific $B$ cells showed a class-switched phenotype by downregulation of IgD and switch from IgM to IgG. The enrichment of class-switched B cells is in agreement with antigenspecificity of the isolated B cells, since it is well known that encounter of B cells with their specific antigen results in isotype switching and somatic hypermutation (Good-Jacobson and Shlomchik 2010). As expected for antigen-specific B cells that recently underwent activation, MHC and costimulatory molecules were upregulated in OVA- and KLH-specific B cells 
(Lenschow et al. 1994, Nashar and Drake 2005, Rodriguez-Pinto 2005). In line with this, no class-switch or activation was observed in OVA- or KLH-negative B cells in immunized mice. The increase in the percentage of $\mathrm{CD} 138^{+}$antigen-specific $\mathrm{B}$ cells probably represents an intermediate state of a part of the antigen-specific $B$ cell population undergoing differentiation into plasma cells (Lacotte et al. 2013). It was not expected that B220 expression stayed unchanged in antigen-specific B cell compared to control B cells. B220 is suggested to regulate antigen-receptor mediated signal transduction by controlling phosphorylation of multiple compartments (Brown et al. 1994). Moreover, downregulation of B220 has been described previously in antigen-specific B cells of immunized mice (Lalor et al. 1992, Smith et al. 1996) and after in vitro activation of B cells (Dustin et al. 1995). However, the analyzed B cells in those studies were antibody-secreting CD138 ${ }^{+}$B cells. In contrast, the B cells in the present study are mostly CD138 antigen-specific B cells and therefore rather of the classical memory B cell phenotype that expresses B220 (McHeyzerWilliams et al. 2000). The upregulation of CD19 in immunized mice was expected, since it plays an important role in affinity maturation upon antigen encounter and the subsequent antibody response (Del Nagro et al. 2005).

Stimulation with the CD40 ligand and IL-4 was shown to efficiently stimulate murine cells to proliferate and develop an antigen-presenting phenotype (Ahmadi et al. 2008, Liebig et al. 2010). Accordingly, OVA-specific B cells proliferated when cultivated on CD40Lexpressing HeLa cells and showed the expected cluster formation and morphology (Liebig et al. 2010). In addition, B cells could be highly enriched over a period of 14 days by stimulation with the CD40L even when starting with a low B cell purity.

As expected from earlier studies on polyclonal CD40B cells (Ahmadi et al. 2008, Liebig et al. 2010), antigen-specific B cells further upregulate molecules that are involved in antigenpresentation. Furthermore, they downregulate the expression of IgD and upregulate the expression of $\operatorname{lgM}$ and IgG. This change was also observed in polyclonal B cells, thus indicating an induction of class-switching and differentiation into memory $B$ cells or plasma cells (Good-Jacobson and Shlomchik 2010). The slight increase in the percentage of $\mathrm{CD} 138^{+} \mathrm{CD} 40 \mathrm{~B}$ cells after one day in culture was expected, since it was shown that CD40 stimulation has an influence on the differentiation into plasma cells (Spriggs et al. 1992, Grabstein et al. 1993, Maliszewski et al. 1993). B220 expression was slightly changed in 
control CD4OB cells, OVA-specific and OVA-negative CD40B cells. However, this change was not significant. The observed upregulation of CD19 after CD40L stimulation in antigenspecific B cells was expected, since CD19 signaling decreases the threshold for BCR stimulation (Depoil et al. 2008) and plays an important role in particular in class-switched B cells, i.e. deficiency in CD19 signaling leads to an impaired affinity maturation and antibody response (Kanegane et al. 2007, van Zelm et al. 2014).

Unexpectedly, antigen-specific murine B cells could not be expanded in the CD40 culture. The percentage of OVA-specific B cells among the entire B cell population decreased rapidly, even though the initial antigen-specific B cell purity was high. This observation is in line with a study by Ahamdi et al. (Ahmadi et al. 2008), who cultivated B cells from antigenimmunized mice on CD40L-expressing feeder cells and reported a drop in the percentage of antigen-specific B cells in the first days of culture. They did in fact report a stabilization of the percentages of antigen-specific B cells. However, these data were not shown in the publication. Although the number of cells in culture decreased in the first seven days, the loss of antigen-specific B cells due to apoptosis was only minor. Instead, antigen-specific class-switched B cells seem to possess a proliferative disadvantage compared to the polyclonal B cells that did not undergo class-switching. This hypothesis is supported by other studies, which observed that the expansion capacity of murine $\operatorname{lgG}^{+} \mathrm{B}$ was lower than that of $\operatorname{lgM}^{+}$B cells (Kometani et al. 2013). These results were different to studies with human B cells, which do not show a dependence of proliferation on a class-switched phenotype (Tangye et al. 2003).

The question arises why a strong activation occurs in antigen-specific CD4OB cells as seen by upregulation of $\mathrm{MHC}$ and costimulatory markers, although the proliferation is poor. However, several experiments with soluble anti-CD40 antibodies indicate that the activation of $B$ cells is independent of the proliferation especially when using the CD4OL for stimulation (unpublished data).

Several factors might influence the outcome of antigen-specific B cell enrichment: from varying immunization efficiency leading to low antigen-specific B cell frequency or low affinity BCRs, over the grade of antigen biotinylation, to the strength of antigen-BCR crosslinking during purification and the resulting activation signaling before culture (Avalos et al. 2014). Although it remains challenging to control these factors, in this study a reliable and 
efficient method was developed that provides antigen-specific B cell enrichment with stable purity for the production of antigen-presenting B cells.

\subsection{Human Antigen-Specific B Cells can be Isolated from PBMCs and Develop an APC Phenotype when Stimulated with the CD40L}

Various studies reported the detection of antigen-specific B cells in human PBMCs of vaccinated donors, but only few focused on the isolation of those cells (Leyendeckers et al. 1999, Ward et al. 2008, Franz et al. 2011). Most studies relied on expansion and conversion of memory B cells into antibody-secreting plasma cells to monitor B cell responses (Crotty et al. 2003, Buisman et al. 2009, Fecteau et al. 2009, Corti et al. 2010). A recent approach achieved the isolation of human tetanus toxoid-specific memory B cells by use of antigentetramers (Franz et al. 2011) and further confirmed antigen-specificity by demonstrating high affinity binding of the BCR antibodies to its antigen. However, the group did not characterize the phenotype of the isolated B cells, nor did they stimulate their antigenpresenting function.

In this study, antigen-specific B cells were isolated from vaccinated donors and enriched by the use of antigen tetramers. The efficiency of the enrichment highly depended on initial antigen-specific B cell frequency and the number of B cell available for purification. Enrichment of HBV-specific B cells with high initial frequencies resulted in $30 \%$ pure populations, although the overall B cell purity could not be increased. The relatively low frequency of B cells in PBMCs and the limitation of the number of PBMCs that can be obtained from a donor, makes it challenging to increase the enrichment of antigen-specific $B$ cells. Higher B cell frequencies in spleens of mice and the overall higher number of available splenocytes probably account for the differences observed in murine and human antigenspecific B cell enrichment. Nevertheless, the here described enrichment technique achieved higher purities of antigen-specific B cells than formerly described methods (Leyendeckers et al. 1999, Ward et al. 2008).

The isolated antigen-specific B cells express several molecules defining their phenotype. As expected, antigen-specific B cells upregulated IgG1 in comparison to non-specific B cells. At first sight, the slight increase in $\operatorname{IgD}^{+}$in antigen-specific $B$ cells seems astonishing, since 
antigen-specific B cells would be expected to possess a classical memory B cells phenotype (Coico et al. 1983, Ziegner et al. 1994). High expression of IgD would rather point towards a naïve $B$ cell phenotype. However, a closer analysis of the $\operatorname{lgD}^{+}$subtypes revealed a lower percentage of naïve $\operatorname{IgD}^{+} \mathrm{CD} 27^{-} \mathrm{B}$ cells in the antigen-specific $\mathrm{B}$ cell compartment compared to control B cells and HBV-negative B cells. Moreover, another IgD ${ }^{+}$B cell subpopulation has been described that additionally expresses CD27 and thereby resembling neither the classical naïve nor the classical memory B cell phenotype (Klein et al. 1999, van Zelm 2012). These $\lg \mathrm{D}^{+} \mathrm{CD} 27^{+} \mathrm{B}$ cells appear to contain a high frequency of autoreactive cells (Koelsch et al. 2007) and therefore are presumably antigen-specific. Interestingly, in the present study the percentage of $\operatorname{lgD}^{+} \mathrm{CD} 27^{+}$antigen-specific $\mathrm{B}$ cells was increased among the $\operatorname{IgD}^{+}$ subpopulation probably accounting for the observed overall increase in the percentage of $\lg D^{+} B$ cells. The function of this antigen-specific subpopulation is however unclear.

Although antigen-specific B cells of the donors have not been recently activated, they express higher levels of CD86 and MHC II than polyclonal B cells of the same donor. In this context, human memory $B$ cells were shown to express CD86, even after circulating in the body for several years (Good et al. 2009) and unpublished data).

The percentage of $\mathrm{CD} 138^{+}$antigen-specific B cells was increased probably representing an intermediate state of a part of the antigen-specific $B$ cell population undergoing differentiation into plasma blasts or plasma cells (Fink 2012). The upregulation of CD19 expression was expected in human antigen-specific B cells. Like in mice, CD19 is important for affinity maturation and in addition for the development of memory B cells (van Zelm et al. 2014). CD20 was upregulated in antigen-specific B cells. Although CD20 was the one of the first B cell-specific differentiation antigen that was identified (Stashenko et al. 1980), its specific function or ligand are still unknown. Nevertheless, a role in the regulation of $B$ cell activation and proliferation was demonstrated (Tedder et al. 1985) and CD20 was identified to be a component of a multimeric cell surface complex that regulates $\mathrm{Ca}^{2+}$ transport across the plasma membrane (Bubien et al. 1993, Kanzaki et al. 1997). However, its role in antigenspecific B cells is even less well defined. On the one hand, CD20 was shown to be downregulated upon CD40 signaling (Anolik et al. 2003) and to enable optimal B-cell responses, specifically against T-independent antigens (Kuijpers et al. 2010). These observations would be in contrast to the present data, since antigen-specific class-switched 
B cells usually develop through interaction with T cells and subsequent CD40 signaling (Danese et al. 2004, Elgueta et al. 2009). On the other hand, in the context of BCR signaling, CD20 induces calcium flux supporting BCR signaling (Walshe et al. 2008, Franke et al. 2011) and anti-CD20 treatment results in a time-dependent inhibition of the BCR cascade (van de Ven et al. 2012). This rather suggests an important role of CD20 in the BCR signaling and therefore in antigen-specific $B$ cells.

Stimulation with the CD40 ligand and IL-4 was shown to highly stimulate human B cells proliferation and the development of an antigen-presenting phenotype (Schultze et al. 1997, von Bergwelt-Baildon et al. 2004, Liebig et al. 2009). Accordingly, HBV-specific B cells proliferated when cultivated on CD4OL-expressing NIH cells and showed the expected cluster formation and morphology (Liebig et al. 2009). B cell expansion over a period of 14 days was not as high as expected from literature. This difference probably resulted from downsizing the culture conditions in order to adjust to the low number of HBV-specific B cells. This has been shown to impair B cell proliferation in preceding studies (unpublished data). Moreover, the percentage of HBV-specific B cells stayed stable or decreased in culture (data not shown). $\operatorname{lgG}^{+}$antigen-specific $B$ cells should not have a proliferative disadvantage as it was observed for murine $\operatorname{lgG}^{+} \mathrm{B}$ cells (Tangye et al. 2003). However, other factors might as well contribute to the decrease of specific B cells in the culture: Initial HBV-purity was low, overall proliferation was not high. Moreover, the percentage of $\mathrm{CD}_{13}{ }^{+} \mathrm{B}$ cells increased significantly in HBV-specific cultures from day 7 to day 14 , which might indicate a differentiation into plasma cells. Nevertheless, the overall B cells could be highly enriched over a period of 14 days by stimulation with the CD40L even when starting with a low $B$ cell purity.

As expected from earlier studies on polyclonal CD40B cells (Lapointe et al. 2003, Liebig et al. 2009), antigen-specific B cells highly upregulate costimulatory molecules that are involved in antigen-presentation. $B$ cell activation in terms of CD86 and CD80 upregulation was shown to be independent of $B$ cell proliferation. Thus, the observed high activation despite the poor proliferation was not contradictory. Unexpectedly, MHC II molecules stayed stable in polyclonal $B$ cells of non-vaccinated donors and where even downregulated in antigenspecific and polyclonal B cells of vaccinated donors. This effect might also be explained by insufficient culture conditions due to downsizing of the culture system. Whether the 
acquired antigen-presenting phenotype is effective enough to induce an antigen-specific T cell response remains to be examined in human mixed-lymphocyte reactions.

\subsection{Murine Antigen-Specific CD40B Cells Induce an Antigen-Specific T Cell Response In Vitro and In Vivo}

Murine and human $\mathrm{CD} 40 \mathrm{~B}$ cells were shown to present antigen to $\mathrm{T}$ cells, thereby inducing an antigen-specific T cells response (Schultze et al. 1997, von Bergwelt-Baildon et al. 2002, Ahmadi et al. 2008). In vitro induction of a T cell response in autologous MLRs requires TCR signalling through binding to an autoantigen-MHC complex and T-cell activation through costimulation by APCs. In our model, antigen-specific CD40B cells rapidly upregulate the expression of the costimulatory molecules CD80 and CD86 and MHC I and II molecules. Therefore, it was not astonishing that antigen-specific CD40B cells induce significant proliferation and activation of both $\mathrm{CD}^{+}$and $\mathrm{CD} 8^{+}$antigen-specific T cells. In high B-to-T cell ratios, OVA-specific CD4OB cells were superior to polyclonal CD40B cells, thereby supporting the hypothesis that the use of antigen-specific $B$ cells improves antigenpresentation by the $\mathrm{B}$ cells and thereby induction of a $\mathrm{T}$ cell response. The advantage of antigen-specific $B$ cells for the use as antigen-presenting CD40B cells over polyclonal B cells lies in two characteristics provided by antigen uptake via their specific BCR. First, the BCR affinity was shown to be directly proportional to the capacity of B cells to present antigen to $\mathrm{CD}^{+} \mathrm{T}$ cells (Rodriguez-Pinto 2005), i.e. B cells with a very high affinity BCR ( $\mathrm{K}_{\mathrm{a}}$ of $5 \times 10^{10} \mathrm{M}^{-}$

$\left.{ }^{1}\right)$ could induce $\mathrm{CD}^{+} \mathrm{T}$ cell proliferation after being incubated with soluble antigen at concentrations as low as $0.05 \mathrm{nM}$ (Batista and Neuberger 1998). In the present study, the antigen concentration for pulsing of APCs was 1500 times higher (75 nM). Nevertheless, presentation after fluid-phase pinocytosis of antigen, as it occurs in polyclonal B cells, required concentrations about 5000 times higher (Batista and Neuberger 1998).

Proliferation and activation by APCs was equally high in $\mathrm{CD}^{+}$and $\mathrm{CD} 8^{+} \mathrm{T}$ cells. One would expect a higher response in $\mathrm{CD}^{+}{ }^{+} \mathrm{T}$ cells, since presentation of exogenous antigens via $\mathrm{MHC}$ II molecules is the classical pathway in professional APCs. However, the process of crosspresentation has been described in dendritic cells and B cells (de Wit et al. 2010, Kurts et al. 2010), which allow professional APCs to process and present exogenously derived antigens 
on $\mathrm{MHC}$ class I molecules. Since $\mathrm{CD} 40 \mathrm{~B}$ cells were also observed to upregulate the expression of $\mathrm{MHCI}$ molecules in the culture, it is no longer surprising that $\mathrm{CD}^{+} \mathrm{T}$ cells show equally high responses. In lower B-to-T cell ratios OVA-specific CD40B cells were still more potent in T cell induction than polyclonal CD40B cells. DCs are usually considered to be more potent APCs than B cells (Salio et al. 2001). Therefore, it was astonishing that there was no difference between OVA-specific CD4OB cells and DCs in higher APC-to-T cell ratios. As expected, differences between DCs and CD40B cells became apparent in lower APC-to-T cell ratios. However, from a biological aspect it might be delusive to directly compare two such fundamentally differing cell types. Murine DCs posses a 4.5 times higher surface than CD4OB cells (data not shown) and therefore express more costimulatory and MHC molecules on their surface. Since both DCs and CD40B cells were shown to make contact with more than one $T$ cell at a time (Klein-Gonzalez et al. accepted 2015), a higher surface might lead to an advantage in antigen presentation when the same numbers of APCs are compared in the system. Nevertheless, from the aspect of clinical application it is of high relevance that antigen-specific CD40B cells are equally potent APCs as DCs, especially when considering the fact that, with regard to the clinical application as cellular adjuvant, CD40B cells in contrast to DCs are available at almost unlimited numbers.

Very few studies have focused on the in vivo antigen-presenting capacity of CD40B cells. Two independent reported that B cell activated with a soluble anti-CD40 antibody (HM40-3) alone or in combination with CpG are weak inducers of cytotoxic T cell responses (Lee et al. 2008, Guo et al. 2009). However, two different studies contradicted these results by showing that polyclonal CD4OB cells induced LCMV-specific $\mathrm{CD}^{+} \mathrm{T}$ cell responses after i.p. vaccination (Ritchie et al. 2004, Liebig et al. 2011). In the present study, OVA-specific CD40B cells induced antigen-specific killing of target cells. Although antigen-specific B cells were only enriched for $\mathrm{CD}_{19}{ }^{+} \mathrm{B}$ cells and not for their antigen-specificity, therefore representing only 2 $\%$ of the $B$ cell population, they induced a significantly higher cytotoxic $T$ cell response than polyclonal CD40B cells. Vaccination with enriched OVA-specific CD40B cells would probably result in even stronger $T$ cell responses. In order to match conditions of the antigen-specific CD40B cells (i.e. 2 \% OVA-specific B cells with $98 \%$ polyclonal B cells), mature DCs were mixed with polyclonal $\mathrm{CD} 40 \mathrm{~B}$ cells before injection. Interestingly, this combination did result in only very weak $\mathrm{CD}^{+} \mathrm{T}$ cell induction, which was significantly lower than after vaccination 
with antigen-specific CD40B cells. Only when $95 \%$ pure DCs were injected for vaccination, equally high or higher specific lysis than with OVA-specific CD40B cells was observed. Polyclonal CD40B cells might exert some inhibiting functions on DCs in the mixture (Tretter et al. 2008), thereby accounting for the observed weak induction of T cell responses.

Taken together, these data provide evidence for the capacity of CD40B cells to induce $\mathrm{T}$ cell responses in vitro and in vivo and that antigen-specific CD4OB cells are indeed more efficient APCs than polyclonal CD40B cells.

\subsection{Tumorantigen-Specific CD40B Cells Home to Secondary Lymphoid Organs and to the Tumor}

An APC must physically encounter a T cell in order to induce an immune response. This takes place in the secondary lymphoid organs. In the setting of the anti-tumor immune response these interactions usually must occur in the tumor draining lymph nodes in order to achieve a strong response. The in vivo migration experiments confirmed homing of polyclonal and antigen-specific $\mathrm{CD} 40 \mathrm{~B}$ cells into the spleen and the abdominal lymph nodes. A five-day period seems to be sufficient for the CD4OB cells to arrive in the secondary lymphoid organs after i.v. injection. This is in line with another study by Ahmadi et al., who could detect GFP ${ }^{+}$ CD40B cells of immunized mice in the spleen and lymph nodes seven days after injection by flow cytometry analyses (Ahmadi et al. 2008). After s.c. injection polyclonal CD40B cells were not detected by luciferase imaging. Therefore, s.c. injection was not included in subsequent experiments. However, a recent study with $\mathrm{GFP}^{+}$cells showed that a small number of polyclonal CD40B cells homes to the secondary lymphoid organs after s.c. injection (KleinGonzalez et al. accepted 2015). The luciferase detection method is probably not sensitive enough to detect this small number of cells. In the lymphoid tissue, CD40B cells were recently shown to accumulate in the B-cell follicles, but also to a small extend in the T-cell zone or at the edge of the T-cell zone (Klein-Gonzalez et al. accepted 2015). These results suggest that $\mathrm{CD} 40 \mathrm{~B}$ cells interact with $\mathrm{T}$ cells in the lymphoid organs. These findings imply a clear advantage of CD4OB cells over DCs, of which only $4 \%$ of injected cells migrate to the draining lymph nodes in mice and humans and rather stay at the site of injection (Steinman and Banchereau 2007). 
As expected, polyclonal CD40B cells do not home to the tumor in E.G7 tumor-bearing mice, but localize in the spleen, the abdominal lymph nodes and the tumor draining lymph nodes. Excitingly, tumorantigen-specific CD4OB cells appeared in the tumor three days after i.v. injection while about half of the $\mathrm{LuC}^{+} \mathrm{CD} 40 \mathrm{~B}$ cells remained in the spleen and the tumor draining lymph nodes. Tumor-infiltrating B cells in human breast cancer (Hansen et al. 2001) and B cells in the tumor-draining lymph nodes in mice (Li et al. 2009) were shown to produce autoantibodies against tumor targets, thereby suggesting that at least some of the tumor infiltrating B cells are antigen-specific. Tumor-infiltrating B cells also contribute to tumor cell death by expression of granzyme B (Hagn et al. 2009) and TRAIL (Kemp et al. 2004), an apoptosis inducing protein. Therefore, it was not completely unexpected to detect tumorantigen-specific $\mathrm{CD} 40 \mathrm{~B}$ cells in the tumor. These findings offer new application possibilities for $\mathrm{CD} 40 \mathrm{~B}$ cells in cancer immunotherapy. Loading of tumorantigen-specific CD40B cells with magnetic beads that can be detected in MRI could allow early detection of tumors. Moreover, tumorantigen-specific CD4OB cells could be used as drug delivery vehicle by loading them with oncolytic viruses.

\subsection{Antigen-Specific B Cells can be Stimulated to Differentiate into Antibody- Secreting Plasma Cells}

Several protocols have been reported to differentiate B cells into antibody-secreting plasma cells (Spriggs et al. 1992, Grabstein et al. 1993, Maliszewski et al. 1993, Kawabe et al. 1994, Arpin et al. 1995, Callard et al. 1995, Han et al. 1995, Silvy et al. 1996, Randall et al. 1998, Ozaki et al. 2004, Kometani et al. 2013). Most of these protocols have been focusing on B cells in general rather than antigen-specific B cells, therefore leaving the class-switched phenotype without consideration. However, at least one study reported the differentiation of antigen-experienced $\operatorname{lgG}^{+} \mathrm{B}$ cells into plasma cells after stimulation with the BCR ligand (Kometani et al. 2013). In the present study, antigen-specific B cells were therefore stimulated with crosslinked OVA-protein in order to trigger differentiation into plasma cells. The combination of OVA-protein, IL-4, IL-21 and soluble CD40L induced expression of CD138, a plasma cells marker, in about stimulated $50 \%$ of B cells. The crucial influence of IL-21 was not surprising, since this cytokines was reported in other studies to be essential for plasma cell differentiation (Ozaki et al. 2004). However, the influence of CD40L was unexpected, 
because contradictory results have been reported on its role in plasma cell differentiation. While several in vitro studies have suggested that CD40 signaling promotes B cell differentiation and Ig secretion (Spriggs et al. 1992, Grabstein et al. 1993, Maliszewski et al. 1993), in vitro and in vivo studies in CD40-deficient mice have suggested that CD40 engagement may actively inhibit B cell differentiation into plasma cells (Kawabe et al. 1994, Arpin et al. 1995, Callard et al. 1995, Han et al. 1995, Silvy et al. 1996, Randall et al. 1998). However, those studies focused on differentiation of naïve B cells. In contrast, at least one study supported the observation that CD40L stimulation is beneficial for differentiation of antigen-experienced B cells into plasma cells (Kometani et al. 2013). Therefore, these contradictory results might simply display the difference between naïve B cells and antigenexperienced $\operatorname{lgG}^{+} \mathrm{B}$ cells. Although LPS, which was used as positive control in differentiation assays, induced stronger differentiation into $\mathrm{CD} 138^{+}$cells and higher antibody secretion than other stimuli, it was not considered as stimulus for in vivo experiments. B cells that were activated with bacterial stimuli were shown to possess regulatory functions (Tretter et al. 2008). Since only about $60 \%$ of B cells in the assay differentiated into plasma cells when stimulated with LPS, the risk was too high to induce immunosuppression rather than an antitumor response when using LPS-differentiated cells for immunotherapy. Moreover, injection of LPS is a known to induce a septic shock in humans and mice (Opal 2010).

Interestingly, beside LPS-treated cells the highest amount of OVA-specific IgG1 antibodies was secreted by cells that were treated with IgM and IL-4. This was unexpected since only about $12 \%$ of IgM and IL-4 treated B cells differentiated into CD $138^{+}$cells. However, this discrepancy can probably be explained by the existence of early antibody-secreting cells, so called plasma blasts, that express MHC class II on their surface, but not yet CD138 (Manz et al. 1998, Hoyer et al. 2004, Racine et al. 2011, Lacotte et al. 2013). However, this hypothesis has to be confirmed by flow cytometry analyses of antibody-secreting cells in differentiation assays. The secretion of $\lg G 1$, the most frequent antibody isotype in human serum and probably the major effector of antigen clearance (Collins and Jackson 2013), by differentiated cells could be established in this study. However, the secretion of other antibody isotypes than IgG1 remains to be confirmed. 


\subsection{Immunotherapy with Antigen-Specific CD40B Cells in Combination with Plasma Cells Leads to Anti-Tumor Immunity and Prolongs Survival}

So far antigen-specific CD40B cells proved to be superior APCs to polyclonal CD40B cells in vitro and in vivo. Therefore, their ability to induce anti-tumor immunity in a preventive vaccination approach was examined. With the expectation to enhance the anti-tumor immune response, cellular and humoral immune functions of $B$ cells were joined by vaccinating mice with antigen-specific CD40B cells as APCS in combination with antibodysecreting plasma cells. The vaccination with tumorantigen-specific CD40B cells induced antitumor immunity, which resulted in the delayed growth of E.G7 lymphomas and a prolonged survival of tumor-bearing mice. Excitingly, this response could be further improved with the combinatorial vaccination of tumorantigen-specific CD4OB cells and anti-tumor antibodysecreting plasma cells. The differences in tumor growth and survival after combined immunotherapy were significant to all other tested cell types and conditions. Polyclonal $\mathrm{CD} 40 \mathrm{~B}$ cells were also shown to reduce tumor growth and prolong survival of tumor-bearing mice. These data are supported by two studies that use CD40B cells for preventive vaccination in LL-LCMV tumors or B16 melanomas (Ritchie et al. 2004, Liebig et al. 2011) reporting a reduced tumor growth. In the later study, $1 \times 10^{7} \mathrm{CD} 40 \mathrm{~B}$ cells were injected per vaccination, which is 100 times more than were used here. It seems that even at low numbers, $C D 40 B$ cells overcome inhibitory mechanisms by the tumor. This idea is also supported by a study on tumor-mediated immune suppression of CD40B cells. Phenotype, migratory potential and antigen-presenting function of human CD40B cells were shown to be resistant to PGE-2, IL-10, TGF- $\beta$ and VEGF (Shimabukuro-Vornhagen et al. 2012). Tumor growth in mice vaccinated with peptide-pulsed DCs was reduced and survival prolonged, however, not as efficiently as in mice that were treated with antigen-specific CD4OB cells alone or in combination with plasma cells. This was unexpected, since so far in vivo CD4OB cells have never been shown to be equal or superior to DCs as cellular adjuvants for immunotherapy.

Whether antigen-specific CD40B cells or the combined immunotherapy with plasma cells will be efficient when used in a therapeutic approach remains to be examined. Guo et al. reported failure of $1 \times 10^{5}$ OVA-peptide pulsed CD4OB cells to reduce tumor growth of E.G7 lymphomas in a therapeutic setting (Guo et al. 2009). Furthermore, Lee et al. showed no 
reduction of tumor growth when treating B16 melanoma-bearing mice with $4 \times 10^{5}$ RNA and costimulatory-transfected CD40B cells (Lee et al. 2008). However, these studies used a soluble anti-CD40 antibody (HM40-3) for the activation of B cells, which was demonstrated to result in a weaker CD40 stimulus than activation by CD40L-expressing feeder cells (Fanslow et al. 1994, Neron et al. 2011). When comparing the APC phenotype of the CD40B cells in the studies of Lee et al. and Guo et al. with those obtained here, it becomes apparent that stimulation with anti-CD40 antibodies does not result in the high upregulation of MHC and costimulatory molecules that were observed in the present study. Therefore, it is likely that in a therapeutic setting antigen-specific CD40B cells will prove to induce anti-tumor immunity anyhow.

\subsection{Concluding Remarks}

Taken together, this study took the first step towards a use of human antigen-specific CD4OB cells as cellular adjuvant in cancer immunotherapy. Isolation and expansion of CD4OB cells is simple and fast resulting in high numbers of $\mathrm{CD} 40 \mathrm{~B}$ cells for adoptive transfer. Thus, they provide several advantages over dendritic cell vaccines. Although human antigen-specific $B$ cells could not yet be expanded in the CD40 culture system, they were highly activated and acquired an antigen-presenting phenotype. Moreover, a mixture of highly expanding polyclonal B cells and few tumorantigen-specific CD40B cells is probably still advantageous for $T$ cell induction, since polyclonal $B$ cells on their own where shown to be efficient antigen-presenting cells. Although the combined preventive vaccination with antigenspecific $C D 40 B$ cells and plasma cells resulted in prolonged survival of mice, it remains to be proven that they have an influence on tumor growth in a therapeutic setting. Treatment with CD40B cells could also be combined with checkpoint blockade, i.e. anti-PD-L1 or antiCXCL4 antibodies, to further enhance the anti-tumor immunity. The next steps that need to be taken into the direction of a clinical application are the isolation of tumorantigen-specific B cells from cancer patients. One major challenge for the application of CD40B cells in the clinic was recently solved by the development of a soluble CD40L that induces both proliferation and activation of human B cells (Garcia-Marquez et al. 2014). 
Another exciting property of human and murine $\mathrm{CD} 40 \mathrm{~B}$ cells, which might be related to their APC function, was recently discovered. CD40B cells form long extensions to T cells and other CD40B cells when incubated together in a 3D-matrixgel (Fig. 4.1).
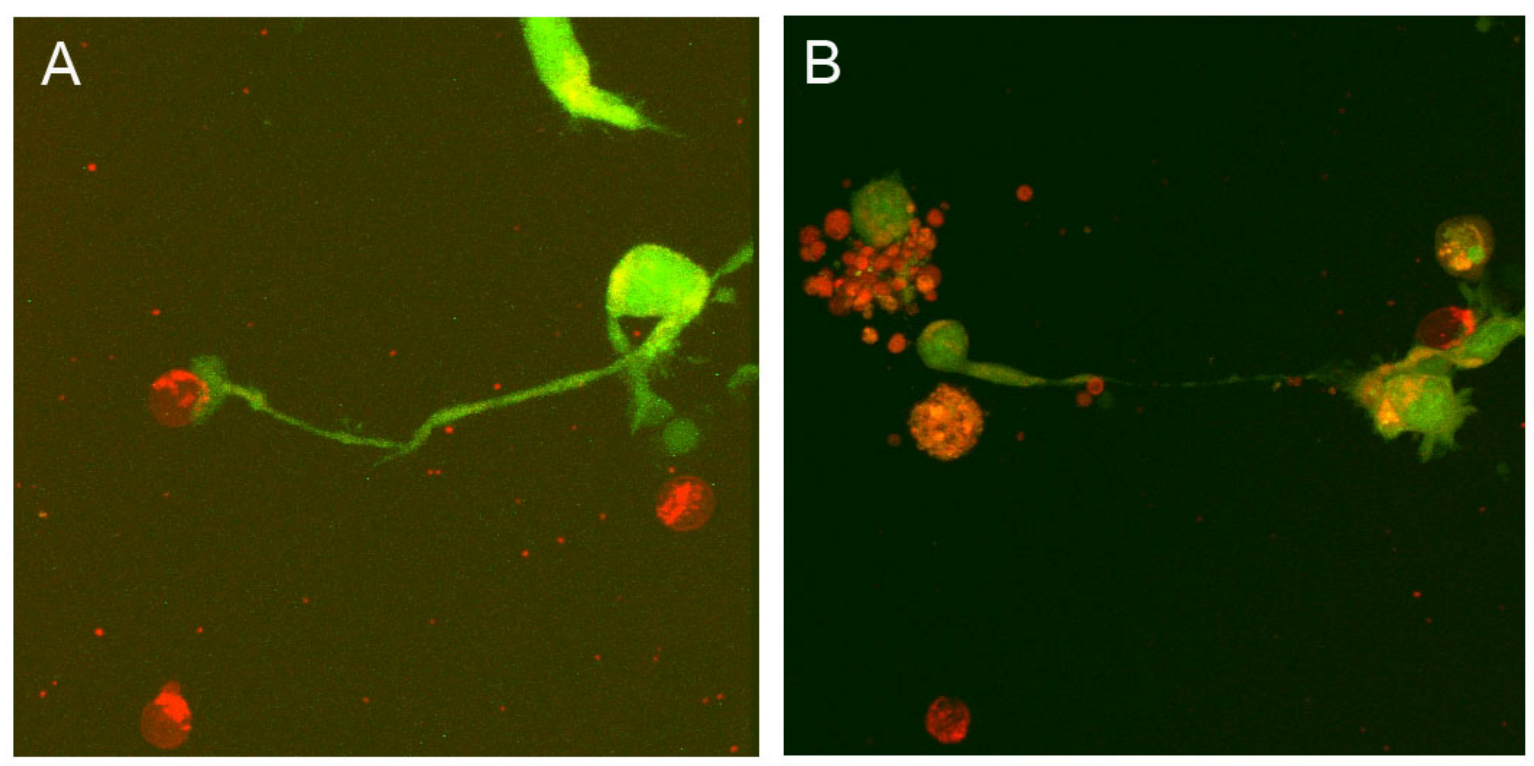

Fig. 4.1 Human CD40B cells form nanotubes. CD40B cells (green) were incubated in 3D-matrigel together with human IL-2-stimulated $\mathrm{CD}^{+} \mathrm{T}$ cells (red). Images were taken with a confocal microscope using a $\times 63$ objective and an appropriate filter set.

These kind of extensions were observed in other types of immune cells including $T$ cells (Sowinski et al. 2008), NK cells (Chauveau et al. 2010), EBV-transfected B cells (Rainy et al. 2013) and others (Zhang 2011) with varying functions. A role of nanotubes in antigenpresentation has not yet been shown. However, the fact that CD40B cells establish these connections with $T$ cells and that the shape of the connection point resembles the shape of an immunological synapse (Robbiati and Guadagnini unpublished data) hints very much to the involvement in antigen-presentation. However, more detailed analyses need to be performed in order to reveal their function in B cell-T cell interaction. 
References 



\section{References}

Abbas, A. K., S. Haber and K. L. Rock (1985). "Antigen presentation by hapten-specific B lymphocytes. II. Specificity and properties of antigen-presenting B lymphocytes, and function of immunoglobulin receptors." J Immunol 135(3): 1661-1667.

Ahmadi, T., A. Flies, Y. Efebera and D. H. Sherr (2008). "CD40 Ligand-activated, antigenspecific $B$ cells are comparable to mature dendritic cells in presenting protein antigens and major histocompatibility complex class I- and class II-binding peptides." Immunology 124(1): 129-140.

Albert, M. L., M. Jegathesan and R. B. Darnell (2001). "Dendritic cell maturation is required for the cross-tolerization of CD8+ T cells." Nat Immunol 2(11): 1010-1017.

Albert, M. L., B. Sauter and N. Bhardwaj (1998). "Dendritic cells acquire antigen from apoptotic cells and induce class I-restricted CTLs." Nature 392(6671): 86-89.

Alfonso, C., M. Liljedahl, O. Winqvist, C. D. Surh, P. A. Peterson, W. P. Fung-Leung, et al. (1999). "The role of H2-O and HLA-DO in major histocompatibility complex class II-restricted antigen processing and presentation." Immunol Rev 172: 255-266.

Allman, D. and S. Pillai (2008). "Peripheral B cell subsets." Curr Opin Immunol 20(2): 149-157.

Allman, D., B. Srivastava and R. C. Lindsley (2004). "Alternative routes to maturity: branch points and pathways for generating follicular and marginal zone B cells." Immunol Rev 197: 147-160.

Anolik, J., R. J. Looney, A. Bottaro, I. Sanz and F. Young (2003). "Down-regulation of CD20 on B cells upon CD40 activation." Eur J Immunol 33(9): 2398-2409.

Arpin, C., J. Dechanet, C. Van Kooten, P. Merville, G. Grouard, F. Briere, et al. (1995). "Generation of memory B cells and plasma cells in vitro." Science 268(5211): 720-722.

Askew, D., J. Gatewood, E. Olivas, K. Havenith and W. S. Walker (1995). "A subset of splenic macrophages process and present native antigen to naive antigen-specific CD4+ T-cells from mice transgenic for an alpha beta T-cell receptor." Cell Immunol 166(1): 62-70.

Attanavanich, K. and J. F. Kearney (2004). "Marginal zone, but not follicular B cells, are potent activators of naive CD4 T cells." J Immunol 172(2): 803-811. 
Avalos, A. M., A. M. Bilate, M. D. Witte, A. K. Tai, J. He, M. P. Frushicheva, et al. (2014). "Monovalent engagement of the BCR activates ovalbumin-specific transnuclear B cells." J Exp Med 211(2): 365-379.

Banchereau, J., F. Briere, C. Caux, J. Davoust, S. Lebecque, Y. J. Liu, et al. (2000). "Immunobiology of dendritic cells." Annu Rev Immunol 18: 767-811.

Banchereau, J., P. de Paoli, A. Valle, E. Garcia and F. Rousset (1991). "Long-term human B cell lines dependent on interleukin-4 and antibody to CD40." Science 251(4989): 70-72.

Banchereau, J. and A. K. Palucka (2005). "Dendritic cells as therapeutic vaccines against cancer." Nat Rev Immunol 5(4): 296-306.

Banchereau, J. and F. Rousset (1991). "Growing human B lymphocytes in the CD40 system." Nature 353(6345): 678-679.

Banchereau, J. and R. M. Steinman (1998). "Dendritic cells and the control of immunity." Nature 392(6673): 245-252.

Batista, F. D. and M. S. Neuberger (1998). "Affinity dependence of the B cell response to antigen: a threshold, a ceiling, and the importance of off-rate." Immunity 8(6): 751-759.

Berard, F., P. Blanco, J. Davoust, E. M. Neidhart-Berard, M. Nouri-Shirazi, N. Taquet, et al. (2000). "Cross-priming of naive CD8 T cells against melanoma antigens using dendritic cells loaded with killed allogeneic melanoma cells." J Exp Med 192(11): 1535-1544.

Bevan, M. J. (2004). "Helping the CD8(+) T-cell response." Nat Rev Immunol 4(8): 595-602.

Bielas, J. H., K. R. Loeb, B. P. Rubin, L. D. True and L. A. Loeb (2006). "Human cancers express a mutator phenotype." Proc Natl Acad Sci U S A 103(48): 18238-18242.

Bishop, G. A., C. R. Moore, P. Xie, L. L. Stunz and Z. J. Kraus (2007). "TRAF proteins in CD40 signaling." Adv Exp Med Biol 597: 131-151.

Boczkowski, D., S. K. Nair, D. Snyder and E. Gilboa (1996). "Dendritic cells pulsed with RNA are potent antigen-presenting cells in vitro and in vivo." J Exp Med 184(2): 465-472.

Bos, J. L. (1989). "ras oncogenes in human cancer: a review." Cancer Res 49(17): 4682-4689.

Bourgeois, C., H. Veiga-Fernandes, A. M. Joret, B. Rocha and C. Tanchot (2002). "CD8 lethargy in the absence of CD4 help." Eur J Immunol 32(8): 2199-2207. 
Bremnes, R. M., K. Al-Shibli, T. Donnem, R. Sirera, S. Al-Saad, S. Andersen, et al. (2011). "The role of tumor-infiltrating immune cells and chronic inflammation the tumor site on cancer development, progression, and prognosis: emphasis on non-small cell lung cancer." J Thorac Oncol 6(4): 824-833.

Bretscher, P. A. (1999). "A two-step, two-signal model for the primary activation of precursor helper T cells." Proc Natl Acad Sci U S A 96(1): 185-190.

Broere, Apasov, Sitkovsky and v. Eden (2011). T cell subsets and T cell-mediated immunity, Birkhäuser Basel.

Brown, V. K., E. W. Ogle, A. L. Burkhardt, R. B. Rowley, J. B. Bolen and L. B. Justement (1994). "Multiple components of the $B$ cell antigen receptor complex associate with the protein tyrosine phosphatase, CD45." J Biol Chem 269(25): 17238-17244.

Bubien, J. K., L. J. Zhou, P. D. Bell, R. A. Frizzell and T. F. Tedder (1993). "Transfection of the CD20 cell surface molecule into ectopic cell types generates a Ca2+ conductance found constitutively in B lymphocytes." J Cell Biol 121(5): 1121-1132.

Buisman, A. M., C. G. de Rond, K. Ozturk, H. I. Ten Hulscher and R. S. van Binnendijk (2009). "Long-term presence of memory B-cells specific for different vaccine components." Vaccine 28(1): 179-186.

Burnet, F. M. (1970). "The concept of immunological surveillance." Prog Exp Tumor Res 13: $1-27$.

Burnet, F. M. (1971). "Immunological surveillance in neoplasia." Transplant Rev 7: 3-25.

Caballero, O. L. and Y. T. Chen (2009). "Cancer/testis (CT) antigens: potential targets for immunotherapy." Cancer Sci 100(11): 2014-2021.

Callard, R. E., J. Herbert, S. H. Smith, R. J. Armitage and K. E. Costelloe (1995). "CD40 crosslinking inhibits specific antibody production by human B cells." Int Immunol 7(11): 18091815.

Carbone, E., G. Ruggiero, G. Terrazzano, C. Palomba, C. Manzo, S. Fontana, et al. (1997). "A new mechanism of NK cell cytotoxicity activation: the CD40-CD40 ligand interaction." J Exp Med 185(12): 2053-2060.

Carter, R. H. and R. Myers (2008). "Germinal center structure and function: lessons from CD19." Semin Immunol 20(1): 43-48. 
Cassell, D. J. and R. H. Schwartz (1994). "A quantitative analysis of antigen-presenting cell function: activated $B$ cells stimulate naive CD4 T cells but are inferior to dendritic cells in providing costimulation." J Exp Med 180(5): 1829-1840.

Caux, C., C. Dezutter-Dambuyant, D. Schmitt and J. Banchereau (1992). "GM-CSF and TNFalpha cooperate in the generation of dendritic Langerhans cells." Nature 360(6401): 258261.

Chames, P., M. Van Regenmortel, E. Weiss and D. Baty (2009). "Therapeutic antibodies: successes, limitations and hopes for the future." Br J Pharmacol 157(2): 220-233.

Chauveau, A., A. Aucher, P. Eissmann, E. Vivier and D. M. Davis (2010). "Membrane nanotubes facilitate long-distance interactions between natural killer cells and target cells." Proc Natl Acad Sci U S A 107(12): 5545-5550.

Cheever, M. A. (2008). "Twelve immunotherapy drugs that could cure cancers." Immunol Rev 222: 357-368.

Cheever, M. A. and C. S. Higano (2011). "PROVENGE (Sipuleucel-T) in prostate cancer: the first FDA-approved therapeutic cancer vaccine." Clin Cancer Res 17(11): 3520-3526.

Chen, Q., V. Daniel, D. W. Maher and P. Hersey (1994). "Production of IL-10 by melanoma cells: examination of its role in immunosuppression mediated by melanoma." Int J Cancer 56(5): 755-760.

Chesnut, R. W., S. M. Colon and H. M. Grey (1982). "Requirements for the processing of antigens by antigen-presenting $B$ cells. I. Functional comparison of $B$ cell tumors and macrophages." J Immunol 129(6): 2382-2388.

Chiba, I., T. Takahashi, M. M. Nau, D. D'Amico, D. T. Curiel, T. Mitsudomi, et al. (1990). "Mutations in the p53 gene are frequent in primary, resected non-small cell lung cancer. Lung Cancer Study Group." Oncogene 5(10): 1603-1610.

Coico, R. F., B. S. Bhogal and G. J. Thorbecke (1983). "Relationship of germinal centers in lymphoid tissue to immunologic memory. VI. Transfer of B cell memory with lymph node cells fractionated according to their receptors for peanut agglutinin." J Immunol 131(5): 2254-2257.

Collins, A. M. and K. J. Jackson (2013). "A Temporal Model of Human IgE and IgG Antibody Function." Front Immunol 4: 235. 
Corti, D., J. P. Langedijk, A. Hinz, M. S. Seaman, F. Vanzetta, B. M. Fernandez-Rodriguez, et al. (2010). "Analysis of memory B cell responses and isolation of novel monoclonal antibodies with neutralizing breadth from HIV-1-infected individuals." PLoS One 5(1): e8805.

Coughlin, C. M., B. A. Vance, S. A. Grupp and R. H. Vonderheide (2004). "RNA-transfected CD40-activated $B$ cells induce functional T-cell responses against viral and tumor antigen targets: implications for pediatric immunotherapy." Blood 103(6): 2046-2054.

Couzin-Frankel, J. (2013). "Breakthrough of the year 2013. Cancer immunotherapy." Science 342(6165): 1432-1433.

Crotty, S., P. Felgner, H. Davies, J. Glidewell, L. Villarreal and R. Ahmed (2003). "Cutting edge: long-term B cell memory in humans after smallpox vaccination." J Immunol 171(10): 49694973.

Danese, S., M. Sans and C. Fiocchi (2004). "The CD40/CD40L costimulatory pathway in inflammatory bowel disease." Gut 53(7): 1035-1043.

de Wit, J., Y. Souwer, T. Jorritsma, H. Klaasse Bos, A. ten Brinke, J. Neefjes, et al. (2010). "Antigen-specific $B$ cells reactivate an effective cytotoxic $T$ cell response against phagocytosed Salmonella through cross-presentation." PLoS One 5(9): e13016.

Del Nagro, C. J., D. C. Otero, A. N. Anzelon, S. A. Omori, R. V. Kolla and R. C. Rickert (2005). "CD19 function in central and peripheral B-cell development." Immunol Res 31(2): 119-131.

Denzin, L. K., J. L. Fallas, M. Prendes and W. Yi (2005). "Right place, right time, right peptide: DO keeps DM focused." Immunol Rev 207: 279-292.

Depoil, D., S. Fleire, B. L. Treanor, M. Weber, N. E. Harwood, K. L. Marchbank, et al. (2008). "CD19 is essential for B cell activation by promoting B cell receptor-antigen microcluster formation in response to membrane-bound ligand." Nat Immunol 9(1): 63-72.

Dhodapkar, M. V., R. M. Steinman, M. Sapp, H. Desai, C. Fossella, J. Krasovsky, et al. (1999). "Rapid generation of broad T-cell immunity in humans after a single injection of mature dendritic cells." J Clin Invest 104(2): 173-180.

Diehl, L., A. T. den Boer, S. P. Schoenberger, E. I. van der Voort, T. N. Schumacher, C. J. Melief, et al. (1999). "CD40 activation in vivo overcomes peptide-induced peripheral cytotoxic T-lymphocyte tolerance and augments anti-tumor vaccine efficacy." Nat Med 5(7): 774-779.

Disis, M. L. and M. A. Cheever (1997). "HER-2/neu protein: a target for antigen-specific immunotherapy of human cancer." Adv Cancer Res 71: 343-371. 
Dorner, T., A. Radbruch and G. R. Burmester (2009). "B-cell-directed therapies for autoimmune disease." Nat Rev Rheumatol 5(8): 433-441.

Draube, A., N. Klein-Gonzalez, S. Mattheus, C. Brillant, M. Hellmich, A. Engert, et al. (2011). "Dendritic cell based tumor vaccination in prostate and renal cell cancer: a systematic review and meta-analysis." PLoS One 6(4): e18801.

Dunn, G. P., A. T. Bruce, H. Ikeda, L. J. Old and R. D. Schreiber (2002). "Cancer immunoediting: from immunosurveillance to tumor escape." Nat Immunol 3(11): 991-998.

Dustin, L. B., E. D. Bullock, Y. Hamada, T. Azuma and D. Y. Loh (1995). "Antigen-driven differentiation of naive Ig-transgenic B cells in vitro." J Immunol 154(10): 4936-4949.

Ehrlich (1909). "Ueber den jetzigen Stand der Karzinomforschung." Ned Tijdschr Geneeskd 5 (Part1): 273-290.

Elgueta, R., M. J. Benson, V. C. de Vries, A. Wasiuk, Y. Guo and R. J. Noelle (2009). "Molecular mechanism and function of CD40/CD40L engagement in the immune system." Immunol Rev 229(1): 152-172.

Eliopoulos, A. G. and L. S. Young (2004). "The role of the CD40 pathway in the pathogenesis and treatment of cancer." Curr Opin Pharmacol 4(4): 360-367.

Evans, D. E., M. W. Munks, J. M. Purkerson and D. C. Parker (2000). "Resting B lymphocytes as APC for naive T lymphocytes: dependence on CD40 ligand/CD40." J Immunol 164(2): 688697.

Faassen, A. E., D. P. Dalke, M. T. Berton, W. D. Warren and S. K. Pierce (1995). "CD40-CD40 ligand interactions stimulate B cell antigen processing." Eur J Immunol 25(12): 3249-3255.

Fanslow, W. C., S. Srinivasan, R. Paxton, M. G. Gibson, M. K. Spriggs and R. J. Armitage (1994). "Structural characteristics of CD40 ligand that determine biological function." Semin Immunol 6(5): 267-278.

Fecteau, J. F., A. Roy and S. Neron (2009). "Peripheral blood CD27+ IgG+ B cells rapidly proliferate and differentiate into immunoglobulin-secreting cells after exposure to low CD154 interaction." Immunology 128(1 Suppl): e353-365.

Ferrone, S. and F. M. Marincola (1995). "Loss of HLA class I antigens by melanoma cells: molecular mechanisms, functional significance and clinical relevance." Immunol Today 16(10): 487-494. 
Figdor, C. G., I. J. de Vries, W. J. Lesterhuis and C. J. Melief (2004). "Dendritic cell immunotherapy: mapping the way." Nat Med 10(5): 475-480.

Fink, K. (2012). "Origin and Function of Circulating Plasmablasts during Acute Viral Infections." Front Immunol 3: 78.

Fishman, M. A. and A. S. Perelson (1999). "Th1/Th2 differentiation and cross-regulation." Bull Math Biol 61(3): 403-436.

Fong, L. and E. G. Engleman (2000). "Dendritic cells in cancer immunotherapy." Annu Rev Immunol 18: 245-273.

Fontenot, J. D., M. A. Gavin and A. Y. Rudensky (2003). "Foxp3 programs the development and function of CD4+CD25+ regulatory T cells." Nat Immunol 4(4): 330-336.

Franke, A., G. J. Niederfellner, C. Klein and H. Burtscher (2011). "Antibodies against CD20 or B-cell receptor induce similar transcription patterns in human lymphoma cell lines." PLoS One 6(2): e16596.

Franz, B., K. F. May, Jr., G. Dranoff and K. Wucherpfennig (2011). "Ex vivo characterization and isolation of rare memory B cells with antigen tetramers." Blood 118(2): 348-357.

Fujiwara, H., J. J. Melenhorst, F. El Ouriaghli, S. Kajigaya, M. Grube, G. Sconocchia, et al. (2005). "In vitro induction of myeloid leukemia-specific CD4 and CD8 T cells by CD40 ligandactivated B cells gene modified to express primary granule proteins." Clin Cancer Res 11(12): 4495-4503.

Galvin, F., G. J. Freeman, Z. Razi-Wolf, W. Hall, Jr., B. Benacerraf, L. Nadler, et al. (1992). "Murine B7 antigen provides a sufficient costimulatory signal for antigen-specific and MHCrestricted T cell activation." J Immunol 149(12): 3802-3808.

Garcia-Marquez, M., A. Shimabukuro-Vornhagen, T. Liebig, K. Wennhold, A. Dauben, A. Dzionek, et al. (2014). "A multimerized form of recombinant human CD40 ligand supports long term activation and proliferation of B cells." Cytotherapie.

Gilboa, E. (1999). "The makings of a tumor rejection antigen." Immunity 11(3): 263-270.

Good-Jacobson, K. L. and M. J. Shlomchik (2010). "Plasticity and heterogeneity in the generation of memory $B$ cells and long-lived plasma cells: the influence of germinal center interactions and dynamics." J Immunol 185(6): 3117-3125. 
Good, K. L., D. T. Avery and S. G. Tangye (2009). "Resting human memory B cells are intrinsically programmed for enhanced survival and responsiveness to diverse stimuli compared to naive B cells." J Immunol 182(2): 890-901.

Grabbe, S., S. Beissert, T. Schwarz and R. D. Granstein (1995). "Dendritic cells as initiators of tumor immune responses: a possible strategy for tumor immunotherapy?" Immunol Today 16(3): 117-121.

Grabstein, K. H., C. R. Maliszewski, K. Shanebeck, T. A. Sato, M. K. Spriggs, W. C. Fanslow, et al. (1993). "The regulation of T cell-dependent antibody formation in vitro by CD40 ligand and IL-2." J Immunol 150(8 Pt 1): 3141-3147.

Graf, D., S. Muller, U. Korthauer, C. van Kooten, C. Weise and R. A. Kroczek (1995). "A soluble form of TRAP (CD40 ligand) is rapidly released after T cell activation." Eur J Immunol 25(6): 1749-1754.

Graff, J. N. and E. D. Chamberlain (2015). "Sipuleucel-T in the treatment of prostate cancer: an evidence-based review of its place in therapy." Core Evid 10: 1-10.

Grakoui, A., S. K. Bromley, C. Sumen, M. M. Davis, A. S. Shaw, P. M. Allen, et al. (1999). "The immunological synapse: a molecular machine controlling $T$ cell activation." Science 285(5425): 221-227.

Grammer, A. C., R. Slota, R. Fischer, H. Gur, H. Girschick, C. Yarboro, et al. (2003). "Abnormal germinal center reactions in systemic lupus erythematosus demonstrated by blockade of CD154-CD40 interactions." J Clin Invest 112(10): 1506-1520.

Grey, H. M., S. M. Colon and R. W. Chesnut (1982). "Requirements for the processing of antigen by antigen-presenting B cells. II. Biochemical comparison of the fate of antigen in B cell tumors and macrophages." J Immunol 129(6): 2389-2395.

Grulich, A. E., M. T. van Leeuwen, M. O. Falster and C. M. Vajdic (2007). "Incidence of cancers in people with HIV/AIDS compared with immunosuppressed transplant recipients: a meta-analysis." Lancet 370(9581): 59-67.

Guo, S., J. Xu, W. Denning and Z. Hel (2009). "Induction of protective cytotoxic T-cell responses by a B-cell-based cellular vaccine requires stable expression of antigen." Gene Ther 16(11): 1300-1313.

Haanen, J. B. and T. N. Schumacher (2007). "Vaccine leads to memory loss." Nat Med 13(3): 248-250. 
Hagn, M., E. Schwesinger, V. Ebel, K. Sontheimer, J. Maier, T. Beyer, et al. (2009). "Human B cells secrete granzyme $B$ when recognizing viral antigens in the context of the acute phase cytokine IL-21." J Immunol 183(3): 1838-1845.

Han, S., K. Hathcock, B. Zheng, T. B. Kepler, R. Hodes and G. Kelsoe (1995). "Cellular interaction in germinal centers. Roles of CD40 ligand and B7-2 in established germinal centers." J Immunol 155(2): 556-567.

Hansen, M. H., H. Nielsen and H. J. Ditzel (2001). "The tumor-infiltrating B cell response in medullary breast cancer is oligoclonal and directed against the autoantigen actin exposed on the surface of apoptotic cancer cells." Proc Natl Acad Sci U SA 98(22): 12659-12664.

Hardy, R. R. (2003). "B-cell commitment: deciding on the players." Curr Opin Immunol 15(2): 158-165.

Hardy, R. R., C. E. Carmack, S. A. Shinton, J. D. Kemp and K. Hayakawa (1991). "Resolution and characterization of pro-B and pre-pro-B cell stages in normal mouse bone marrow." J Exp Med 173(5): 1213-1225.

Hardy, R. R. and K. Hayakawa (2001). "B cell development pathways." Annu Rev Immunol 19: 595-621.

Hardy, R. R., Y. S. Li, D. Allman, M. Asano, M. Gui and K. Hayakawa (2000). "B-cell commitment, development and selection." Immunol Rev 175: 23-32.

Hart, D. N. (1997). "Dendritic cells: unique leukocyte populations which control the primary immune response." Blood 90(9): 3245-3287.

Hayakawa, K., R. Ishii, K. Yamasaki, T. Kishimoto and R. R. Hardy (1987). "Isolation of highaffinity memory B cells: phycoerythrin as a probe for antigen-binding cells." Proc Natl Acad Sci U SA 84(5): 1379-1383.

He, Y., H. Qian, Y. Liu, L. Duan, Y. Li and G. Shi (2014). "The roles of regulatory B cells in cancer." J Immunol Res 2014: 215471.

Herberman, R. B., C. W. Reynolds and J. R. Ortaldo (1986). "Mechanism of cytotoxicity by natural killer (NK) cells." Annu Rev Immunol 4: 651-680.

Hoven, M. Y., L. De Leij, J. F. Keij and T. H. The (1989). "Detection and isolation of antigenspecific B cells by the fluorescence activated cell sorter (FACS)." J Immunol Methods $\mathbf{1 1 7 ( 2 ) :}$ 275-284. 
Hoyer, B. F., K. Moser, A. E. Hauser, A. Peddinghaus, C. Voigt, D. Eilat, et al. (2004). "Shortlived plasmablasts and long-lived plasma cells contribute to chronic humoral autoimmunity in NZB/W mice." J Exp Med 199(11): 1577-1584.

Inaba, K., J. P. Metlay, M. T. Crowley and R. M. Steinman (1990). "Dendritic cells pulsed with protein antigens in vitro can prime antigen-specific, MHC-restricted T cells in situ." J Exp Med 172(2): 631-640.

Ivanov, R., T. Aarts, A. Hagenbeek, S. Hol and S. Ebeling (2005). "B-cell expansion in the presence of the novel 293-CD40L-sCD40L cell line allows the generation of large numbers of efficient xenoantigen-free APC." Cytotherapy 7(1): 62-73.

Jacob, J., J. Przylepa, C. Miller and G. Kelsoe (1993). "In situ studies of the primary immune response to (4-hydroxy-3-nitrophenyl)acetyl. III. The kinetics of $\mathrm{V}$ region mutation and selection in germinal center B cells." J Exp Med 178(4): 1293-1307.

Julius, M. H., C. A. Janeway, Jr. and L. A. Herzenberg (1976). "Isolation of antigen-binding cells from unprimed mice. II. Evidence for monospecificity of antigen-binding cells." Eur J Immunol 6(4): 288-292.

Kanegane, H., K. Agematsu, T. Futatani, M. M. Sira, K. Suga, T. Sekiguchi, et al. (2007). "Novel mutations in a Japanese patient with CD19 deficiency." Genes Immun 8(8): 663-670.

Kantor, A. B. and L. A. Herzenberg (1993). "Origin of murine B cell lineages." Annu Rev Immunol 11: 501-538.

Kanwar, R. K., N. Singh, S. Gurudevan and J. R. Kanwar (2011). "Targeting hepatitis B virus and human papillomavirus induced carcinogenesis: novel patented therapeutics." Recent Pat Antiinfect Drug Discov 6(2): 158-174.

Kanzaki, M., M. A. Lindorfer, J. C. Garrison and I. Kojima (1997). "Activation of the calciumpermeable cation channel CD20 by alpha subunits of the Gi protein." J Biol Chem 272(23): 14733-14739.

Kaplan, D. H., V. Shankaran, A. S. Dighe, E. Stockert, M. Aguet, L. J. Old, et al. (1998). "Demonstration of an interferon gamma-dependent tumor surveillance system in immunocompetent mice." Proc Natl Acad Sci U S A 95(13): 7556-7561.

Kappler, J. W., N. Roehm and P. Marrack (1987). "T cell tolerance by clonal elimination in the thymus." Cell 49(2): 273-280. 
Kawabe, T., T. Naka, K. Yoshida, T. Tanaka, H. Fujiwara, S. Suematsu, et al. (1994). "The immune responses in CD40-deficient mice: impaired immunoglobulin class switching and germinal center formation." Immunity 1(3): 167-178.

Kearney, J. F., W. J. Won, C. Benedict, C. Moratz, P. Zimmer, A. Oliver, et al. (1997). "B cell development in mice." Int Rev Immunol 15(3-4): 207-241.

Kemp, T. J., J. M. Moore and T. S. Griffith (2004). "Human B cells express functional TRAIL/Apo-2 ligand after CpG-containing oligodeoxynucleotide stimulation." J Immunol 173(2): 892-899.

Kennedy, M. K., K. M. Mohler, K. D. Shanebeck, P. R. Baum, K. S. Picha, C. A. Otten-Evans, et al. (1994). "Induction of $B$ cell costimulatory function by recombinant murine CD40 ligand." Eur J Immunol 24(1): 116-123.

Kirk, A. D., P. J. Blair, D. K. Tadaki, H. Xu and D. M. Harlan (2001). "The role of CD154 in organ transplant rejection and acceptance." Philos Trans R Soc Lond B Biol Sci 356(1409): 691-702.

Kirk, A. D., D. M. Harlan, N. N. Armstrong, T. A. Davis, Y. Dong, G. S. Gray, et al. (1997). "CTLA4-Ig and anti-CD40 ligand prevent renal allograft rejection in primates." Proc Natl Acad Sci U S A 94(16): 8789-8794.

Kisielow, P., H. Bluthmann, U. D. Staerz, M. Steinmetz and H. von Boehmer (1988). "Tolerance in T-cell-receptor transgenic mice involves deletion of nonmature $\mathrm{CD} 4+8+$ thymocytes." Nature 333(6175): 742-746.

Klee, G. G. (2000). "Human anti-mouse antibodies." Arch Pathol Lab Med 124(6): 921-923.

Klein-Gonzalez, N., K. Wennhold, S. Balkow, E. Kondo, T. Weber, M. Garcia-Marquez, et al. (accepted 2015). "In vitro and in vivo imaging of initial T-B cell interactions in the setting of B cell-based cancer immunotherapy." Oncoimmunology.

Klein, J. and A. Sato (2000). "The HLA system. First of two parts." N Engl J Med 343(10): 702709.

Klein, U., K. Rajewsky and R. Kuppers (1999). "Phenotypic and molecular characterization of human peripheral blood B-cell subsets with special reference to $\mathrm{N}$-region addition and $\mathrm{J}$ kappa-usage in $\mathrm{V}$ kappa J kappa-joints and kappa/lambda-ratios in naive versus memory Bcell subsets to identify traces of receptor editing processes." Curr Top Microbiol Immunol 246: 141-146; discussion 147.

Kodituwakku, A. P., C. Jessup, H. Zola and D. M. Roberton (2003). "Isolation of antigenspecific B cells." Immunol Cell Biol 81(3): 163-170. 
Koelsch, K., N. Y. Zheng, Q. Zhang, A. Duty, C. Helms, M. D. Mathias, et al. (2007). "Mature B cells class switched to IgD are autoreactive in healthy individuals." J Clin Invest 117(6): 15581565.

Kometani, K., R. Nakagawa, R. Shinnakasu, T. Kaji, A. Rybouchkin, S. Moriyama, et al. (2013). "Repression of the transcription factor Bach2 contributes to predisposition of IgG1 memory B cells toward plasma cell differentiation." Immunity 39(1): 136-147.

Kondo, E., L. Gryschok, J. L. Schultze and M. S. von Bergwelt-Baildon (2009). "Using CD40activated B cells to efficiently identify epitopes of tumor antigens." J Immunother 32(2): 157160.

Koopman, G., C. P. Reutelingsperger, G. A. Kuijten, R. M. Keehnen, S. T. Pals and M. H. van Oers (1994). "Annexin V for flow cytometric detection of phosphatidylserine expression on B cells undergoing apoptosis." Blood 84(5): 1415-1420.

Kraal, G. (1992). "Cells in the marginal zone of the spleen." Int Rev Cytol 132: 31-74.

Kuijpers, T. W., R. J. Bende, P. A. Baars, A. Grummels, I. A. Derks, K. M. Dolman, et al. (2010). "CD20 deficiency in humans results in impaired T cell-independent antibody responses." J Clin Invest 120(1): 214-222.

Kurts, C., B. W. Robinson and P. A. Knolle (2010). "Cross-priming in health and disease." Nat Rev Immunol 10(6): 403-414.

Lacotte, S., M. Decossas, C. Le Coz, S. Brun, S. Muller and H. Dumortier (2013). "Early differentiated CD138(high) MHCll+ IgG+ plasma cells express CXCR3 and localize into inflamed kidneys of lupus mice." PLoS One 8(3): e58140.

Lalor, P. A., G. J. Nossal, R. D. Sanderson and M. G. McHeyzer-Williams (1992). "Functional and molecular characterization of single, (4-hydroxy-3-nitrophenyl)acetyl (NP)-specific, IgG1+ B cells from antibody-secreting and memory B cell pathways in the C57BL/6 immune response to NP." Eur J Immunol 22(11): 3001-3011.

Lanzavecchia, A. (1985). "Antigen-specific interaction between T and B cells." Nature 314(6011): 537-539.

Lanzavecchia, A. (1990). "Receptor-mediated antigen uptake and its effect on antigen presentation to class II-restricted T lymphocytes." Annu Rev Immunol 8: 773-793. 
Lapointe, R., A. Bellemare-Pelletier, F. Housseau, J. Thibodeau and P. Hwu (2003). "CD40stimulated $B$ lymphocytes pulsed with tumor antigens are effective antigen-presenting cells that can generate specific T cells." Cancer Res 63(11): 2836-2843.

Larsen, C. P., E. T. Elwood, D. Z. Alexander, S. C. Ritchie, R. Hendrix, C. Tucker-Burden, et al. (1996). "Long-term acceptance of skin and cardiac allografts after blocking CD40 and CD28 pathways." Nature 381(6581): 434-438.

Le, D. T., D. M. Pardoll and E. M. Jaffee (2010). "Cellular vaccine approaches." Cancer J 16(4): 304-310.

Lee, J., C. M. Dollins, D. Boczkowski, B. A. Sullenger and S. Nair (2008). "Activated B cells modified by electroporation of multiple mRNAs encoding immune stimulatory molecules are comparable to mature dendritic cells in inducing in vitro antigen-specific T-cell responses." Immunology 125(2): 229-240.

Lenschow, D. J., A. I. Sperling, M. P. Cooke, G. Freeman, L. Rhee, D. C. Decker, et al. (1994). "Differential up-regulation of the B7-1 and B7-2 costimulatory molecules after Ig receptor engagement by antigen." J Immunol 153(5): 1990-1997.

Leyendeckers, H., M. Odendahl, A. Lohndorf, J. Irsch, M. Spangfort, S. Miltenyi, et al. (1999). "Correlation analysis between frequencies of circulating antigen-specific IgG-bearing memory B cells and serum titers of antigen-specific IgG." European Journal of Immunology 29(4): 1406-1417.

Li, Q., S. Teitz-Tennenbaum, E. J. Donald, M. Li and A. E. Chang (2009). "In vivo sensitized and in vitro activated $B$ cells mediate tumor regression in cancer adoptive immunotherapy." J Immunol 183(5): 3195-3203.

Li, Y., M. N. Wang, H. Li, K. D. King, R. Bassi, H. Sun, et al. (2002). "Active immunization against the vascular endothelial growth factor receptor flk1 inhibits tumor angiogenesis and metastasis." J Exp Med 195(12): 1575-1584.

Liebig, Shimabukuro-Vornhagen, Samir-Ghali and v. Bergwelt-Baildon (2011). In vivo evaluation of a CD40-activated B cell-based tumor vaccine for use in cellular immunotherapy. CIMT 9th Annual Meeting, Mainz.

Liebig, T. M., A. Fiedler, N. Klein-Gonzalez, A. Shimabukuro-Vornhagen and M. von BergweltBaildon (2010). "Murine model of CD40-activation of B cells." J Vis Exp(37).

Liebig, T. M., A. Fiedler, S. Zoghi, A. Shimabukuro-Vornhagen and M. S. von Bergwelt-Baildon (2009). "Generation of human CD40-activated B cells." J Vis Exp(32). 
Liu, Q., C. Zhang, A. Sun, Y. Zheng, L. Wang and X. Cao (2009). "Tumor-educated CD11bhighlalow regulatory dendritic cells suppress $T$ cell response through arginase I." J Immunol 182(10): 6207-6216.

Mackey, M. F., J. R. Gunn, C. Maliszewsky, H. Kikutani, R. J. Noelle and R. J. Barth, Jr. (1998). "Dendritic cells require maturation via CD40 to generate protective antitumor immunity." J Immunol 161(5): 2094-2098.

Mager, D. L. (2006). "Bacteria and cancer: cause, coincidence or cure? A review." J Transl Med 4: 14.

Mahnke, K., E. Schmitt, L. Bonifaz, A. H. Enk and H. Jonuleit (2002). "Immature, but not inactive: the tolerogenic function of immature dendritic cells." Immunol Cell Biol 80(5): 477483.

Mailliard, R. B., A. Wankowicz-Kalinska, Q. Cai, A. Wesa, C. M. Hilkens, M. L. Kapsenberg, et al. (2004). "alpha-type-1 polarized dendritic cells: a novel immunization tool with optimized CTL-inducing activity." Cancer Res 64(17): 5934-5937.

Malati, T. (2007). "Tumour markers: An overview." Indian J Clin Biochem 22(2): 17-31.

Maliszewski, C. R., K. Grabstein, W. C. Fanslow, R. Armitage, M. K. Spriggs and T. A. Sato (1993). "Recombinant CD40 ligand stimulation of murine B cell growth and differentiation: cooperative effects of cytokines." Eur J Immunol 23(5): 1044-1049.

Mantovani, A. and A. Sica (2010). "Macrophages, innate immunity and cancer: balance, tolerance, and diversity." Curr Opin Immunol 22(2): 231-237.

Manz, R. A., M. Lohning, G. Cassese, A. Thiel and A. Radbruch (1998). "Survival of long-lived plasma cells is independent of antigen." Int Immunol 10(11): 1703-1711.

Martin, F. and J. F. Kearney (2002). "Marginal-zone B cells." Nat Rev Immunol 2(5): 323-335.

Martin, F., A. M. Oliver and J. F. Kearney (2001). "Marginal zone and B1 B cells unite in the early response against T-independent blood-borne particulate antigens." Immunity 14(5): 617-629.

Mayordomo, J. I., T. Zorina, W. J. Storkus, L. Zitvogel, C. Celluzzi, L. D. Falo, et al. (1995). "Bone marrow-derived dendritic cells pulsed with synthetic tumour peptides elicit protective and therapeutic antitumour immunity." Nat Med 1(12): 1297-1302. 
Mazzei, G. J., M. D. Edgerton, C. Losberger, S. Lecoanet-Henchoz, P. Graber, A. Durandy, et al. (1995). "Recombinant soluble trimeric CD40 ligand is biologically active." J Biol Chem 270(13): 7025-7028.

McHeyzer-Williams, L. J., M. Cool and M. G. McHeyzer-Williams (2000). "Antigen-specific B cell memory: expression and replenishment of a novel b220(-) memory b cell compartment." J Exp Med 191(7): 1149-1166.

Melchers, F., A. Rolink, U. Grawunder, T. H. Winkler, H. Karasuyama, P. Ghia, et al. (1995). "Positive and negative selection events during B lymphopoiesis." Curr Opin Immunol 7(2): 214-227.

Mellman, I., G. Coukos and G. Dranoff (2011). "Cancer immunotherapy comes of age." Nature 480(7378): 480-489.

Mosmann, T. R., H. Cherwinski, M. W. Bond, M. A. Giedlin and R. L. Coffman (1986). "Two types of murine helper $T$ cell clone. I. Definition according to profiles of lymphokine activities and secreted proteins." J Immunol 136(7): 2348-2357.

Moutai, T., H. Yamana, T. Nojima and D. Kitamura (2014). "A novel and effective cancer immunotherapy mouse model using antigen-specific B cells selected in vitro." PLoS One 9(3): e92732.

Nashar, T. O. and J. R. Drake (2005). "The pathway of antigen uptake and processing dictates MHC class II-mediated B cell survival and activation." J Immunol 174(3): 1306-1316.

Neefjes, J., M. L. Jongsma, P. Paul and O. Bakke (2011). "Towards a systems understanding of MHC class I and MHC class II antigen presentation." Nat Rev Immunol 11(12): 823-836.

Neron, S., P. J. Nadeau, A. Darveau and J. F. Leblanc (2011). "Tuning of CD40-CD154 interactions in human B-lymphocyte activation: a broad array of in vitro models for a complex in vivo situation." Arch Immunol Ther Exp (Warsz) 59(1): 25-40.

Newman, J., J. S. Rice, C. Wang, S. L. Harris and B. Diamond (2003). "Identification of an antigen-specific B cell population." J Immunol Methods 272(1-2): 177-187.

Opal, S. M. (2010). "Endotoxins and other sepsis triggers." Contrib Nephrol 167: 14-24.

Oshiba, A., H. Renz, J. Yata and E. W. Gelfand (1994). "Isolation and characterization of human antigen-specific B lymphocytes." Clin Immunol Immunopathol 72(3): 342-349. 
Osmond, D. G. (1990). "B cell development in the bone marrow." Semin Immunol 2(3): 173180.

Osmond, D. G., A. Rolink and F. Melchers (1998). "Murine B lymphopoiesis: towards a unified model." Immunol Today 19(2): 65-68.

Ozaki, K., R. Spolski, R. Ettinger, H. P. Kim, G. Wang, C. F. Qi, et al. (2004). "Regulation of B cell differentiation and plasma cell generation by IL-21, a novel inducer of Blimp-1 and Bcl6." J Immunol 173(9): 5361-5371.

Palucka, K., H. Ueno, J. Fay and J. Banchereau (2011). "Dendritic cells and immunity against cancer." J Intern Med 269(1): 64-73.

Palucka, K., H. Ueno, L. Roberts, J. Fay and J. Banchereau (2010). "Dendritic cells: are they clinically relevant?" Cancer J 16(4): 318-324.

Parkhurst, M. R., M. L. Salgaller, S. Southwood, P. F. Robbins, A. Sette, S. A. Rosenberg, et al. (1996). "Improved induction of melanoma-reactive CTL with peptides from the melanoma antigen gp100 modified at HLA-A*0201-binding residues." J Immunol 157(6): 2539-2548.

Parungo, C. P., D. I. Soybel, Y. L. Colson, S. W. Kim, S. Ohnishi, A. M. DeGrand, et al. (2007). "Lymphatic drainage of the peritoneal space: a pattern dependent on bowel lymphatics." Ann Surg Oncol 14(2): 286-298.

Paus, D., T. G. Phan, T. D. Chan, S. Gardam, A. Basten and R. Brink (2006). "Antigen recognition strength regulates the choice between extrafollicular plasma cell and germinal center B cell differentiation." J Exp Med 203(4): 1081-1091.

Pearson, T. C., J. Trambley, K. Odom, D. C. Anderson, S. Cowan, R. Bray, et al. (2002). "AntiCD40 therapy extends renal allograft survival in rhesus macaques." Transplantation 74(7): 933-940.

Pereira, J. P., L. M. Kelly and J. G. Cyster (2010). "Finding the right niche: B-cell migration in the early phases of T-dependent antibody responses." Int Immunol 22(6): 413-419.

Peters, A. L., L. L. Stunz and G. A. Bishop (2009). "CD40 and autoimmunity: the dark side of a great activator." Semin Immunol 21(5): 293-300.

Phan, T. G., M. Amesbury, S. Gardam, J. Crosbie, J. Hasbold, P. D. Hodgkin, et al. (2003). "B cell receptor-independent stimuli trigger immunoglobulin (Ig) class switch recombination and production of IgG autoantibodies by anergic self-reactive B cells." J Exp Med 197(7): 845860. 
Popi, A. F., J. D. Lopes and M. Mariano (2004). "Interleukin-10 secreted by B-1 cells modulates the phagocytic activity of murine macrophages in vitro." Immunology 113(3): 348-354.

Quillien, V., A. Moisan, A. Carsin, T. Lesimple, C. Lefeuvre, H. Adamski, et al. (2005). "Biodistribution of radiolabelled human dendritic cells injected by various routes." Eur J Nucl Med Mol Imaging 32(7): 731-741.

Racine, R., M. McLaughlin, D. D. Jones, S. T. Wittmer, K. C. MacNamara, D. L. Woodland, et al. (2011). "IgM production by bone marrow plasmablasts contributes to long-term protection against intracellular bacterial infection." J Immunol 186(2): 1011-1021.

Rainy, N., D. Chetrit, V. Rouger, H. Vernitsky, O. Rechavi, D. Marguet, et al. (2013). "H-Ras transfers from B to T cells via tunneling nanotubes." Cell Death Dis 4: e726.

Ramsay, A. G. (2013). "Immune checkpoint blockade immunotherapy to activate anti-tumour T-cell immunity." Br J Haematol 162(3): 313-325.

Randall, T. D., A. W. Heath, L. Santos-Argumedo, M. C. Howard, I. L. Weissman and F. E. Lund (1998). "Arrest of B lymphocyte terminal differentiation by CD40 signaling: mechanism for lack of antibody-secreting cells in germinal centers." Immunity 8(6): 733-742.

Ranheim, E. A. and T. J. Kipps (1993). "Activated T cells induce expression of B7/BB1 on normal or leukemic B cells through a CD40-dependent signal." J Exp Med 177(4): 925-935.

Ribas, A., L. H. Butterfield, J. A. Glaspy and J. S. Economou (2002). "Cancer immunotherapy using gene-modified dendritic cells." Curr Gene Ther 2(1): 57-78.

Ridolfi, R., A. Riccobon, R. Galassi, G. Giorgetti, M. Petrini, L. Fiammenghi, et al. (2004). "Evaluation of in vivo labelled dendritic cell migration in cancer patients." J Transl Med 2(1): 27.

Ritchie, D. S., J. Yang, I. F. Hermans and F. Ronchese (2004). "B-Lymphocytes activated by CD40 ligand induce an antigen-specific anti-tumour immune response by direct and indirect activation of CD8(+) T-cells." Scand J Immunol 60(6): 543-551.

Robbiati and Guadagnini (unpublished data). "The shape of the immunological synapse." Lymphocyte Cell Biology Unit Institut http://www.pasteur.fr/en/research/immunology/units-groups/lymphocyte-cellbiology/figures. 
Robson, N. C., A. M. Donachie and A. M. Mowat (2008). "Simultaneous presentation and cross-presentation of immune-stimulating complex-associated cognate antigen by antigenspecific B cells." Eur J Immunol 38(5): 1238-1246.

Rodriguez-Pinto, D. (2005). "B cells as antigen presenting cells." Cell Immunol 238(2): 67-75.

Rolink, A. G., E. ten Boekel, T. Yamagami, R. Ceredig, J. Andersson and F. Melchers (1999). "B cell development in the mouse from early progenitors to mature B cells." Immunol Lett 68(1): 89-93.

Romani, N., S. Gruner, D. Brang, E. Kampgen, A. Lenz, B. Trockenbacher, et al. (1994). "Proliferating dendritic cell progenitors in human blood." J Exp Med 180(1): 83-93.

Rosenberg, S. A. (2004). "Shedding light on immunotherapy for cancer." $\underline{N \text { Engl J Med }}$ 350(14): 1461-1463.

Sakaguchi, S. (2005). "Naturally arising Foxp3-expressing CD25+CD4+ regulatory T cells in immunological tolerance to self and non-self." Nat Immunol 6(4): 345-352.

Salio, M., D. Shepherd, P. R. Dunbar, M. Palmowski, K. Murphy, L. Wu, et al. (2001). "Mature dendritic cells prime functionally superior melan-A-specific CD8+ lymphocytes as compared with nonprofessional APC." J Immunol 167(3): 1188-1197.

Sallusto, F. and A. Lanzavecchia (1994). "Efficient presentation of soluble antigen by cultured human dendritic cells is maintained by granulocyte/macrophage colony-stimulating factor plus interleukin 4 and downregulated by tumor necrosis factor alpha." J Exp Med 179(4): 1109-1118.

Sarid, R. and S. J. Gao (2011). "Viruses and human cancer: from detection to causality." Cancer Lett 305(2): 218-227.

Schlosser, H. A., S. Theurich, A. Shimabukuro-Vornhagen, U. Holtick, D. L. Stippel and M. von Bergwelt-Baildon (2014). "Overcoming tumor-mediated immunosuppression." Immunotherapy 6(9): 973-988.

Schreiber, R. D., L. J. Old and M. J. Smyth (2011). "Cancer immunoediting: integrating immunity's roles in cancer suppression and promotion." Science 331(6024): 1565-1570.

Schultze, J. L., S. Grabbe and M. S. von Bergwelt-Baildon (2004). "DCs and CD40-activated B cells: current and future avenues to cellular cancer immunotherapy." Trends Immunol 25(12): 659-664. 
Schultze, J. L., S. Michalak, M. J. Seamon, G. Dranoff, K. Jung, J. Daley, et al. (1997). "CD40activated human B cells: an alternative source of highly efficient antigen presenting cells to generate autologous antigen-specific T cells for adoptive immunotherapy." J Clin Invest 100(11): 2757-2765.

Schwartz, R. H. (2003). "T cell anergy." Annu Rev Immunol 21: 305-334.

Sensi, M. and A. Anichini (2006). "Unique tumor antigens: evidence for immune control of genome integrity and immunogenic targets for $T$ cell-mediated patient-specific immunotherapy." Clin Cancer Res 12(17): 5023-5032.

Shankaran, V., H. Ikeda, A. T. Bruce, J. M. White, P. E. Swanson, L. J. Old, et al. (2001). "IFNgamma and lymphocytes prevent primary tumour development and shape tumour immunogenicity." Nature 410(6832): 1107-1111.

Shen, S., Z. Xu, X. Qian, Y. Ding, L. Yu and B. Liu (2007). "Autogeneic rna-electroporated CD40-ligand activated b-cells from hepatocellular carcinoma patients induce CD8+ T-cell responses ex vivo." Exp Oncol 29(2): 137-143.

Shen, S. N., Z. Xu, X. P. Qian, Y. T. Ding, L. X. Yu and B. R. Liu (2008). "RNA-electroporated CD40-activated B cells induce functional T-cell responses against HepG2 cells." Eur J Cancer Care (Engl) 17(4): 404-411.

Shimabukuro-Vornhagen, A., A. Draube, T. M. Liebig, A. Rothe, M. Kochanek and M. S. von Bergwelt-Baildon (2012). "The immunosuppressive factors IL-10, TGF-beta, and VEGF do not affect the antigen-presenting function of CD40-activated B cells." J Exp Clin Cancer Res 31: 47.

Shortman, K. and Y. J. Liu (2002). "Mouse and human dendritic cell subtypes." Nat Rev Immunol 2(3): 151-161.

Sidiropoulos, P. I. and D. T. Boumpas (2004). "Lessons learned from anti-CD40L treatment in systemic lupus erythematosus patients." Lupus 13(5): 391-397.

Silvy, A., C. Lagresle, C. Bella and T. Defrance (1996). "The differentiation of human memory B cells into specific antibody-secreting cells is CD40 independent." Eur J Immunol 26(3): 517524.

Small, E. J., P. Fratesi, D. M. Reese, G. Strang, R. Laus, M. V. Peshwa, et al. (2000). "Immunotherapy of hormone-refractory prostate cancer with antigen-loaded dendritic cells." J Clin Oncol 18(23): 3894-3903. 
Smith, K. G., T. D. Hewitson, G. J. Nossal and D. M. Tarlinton (1996). "The phenotype and fate of the antibody-forming cells of the splenic foci." Eur J Immunol 26(2): 444-448.

Sorenmo, K. U., E. Krick, C. M. Coughlin, B. Overley, T. P. Gregor, R. H. Vonderheide, et al. (2011). "CD40-activated B cell cancer vaccine improves second clinical remission and survival in privately owned dogs with non-Hodgkin's lymphoma." PLoS One 6(8): e24167.

Sowinski, S., C. Jolly, O. Berninghausen, M. A. Purbhoo, A. Chauveau, K. Kohler, et al. (2008). "Membrane nanotubes physically connect $T$ cells over long distances presenting a novel route for HIV-1 transmission." Nat Cell Biol 10(2): 211-219.

Spriggs, M. K., R. J. Armitage, L. Strockbine, K. N. Clifford, B. M. Macduff, T. A. Sato, et al. (1992). "Recombinant human CD40 ligand stimulates B cell proliferation and immunoglobulin E secretion." J Exp Med 176(6): 1543-1550.

Stashenko, P., L. M. Nadler, R. Hardy and S. F. Schlossman (1980). "Characterization of a human B lymphocyte-specific antigen." J Immunol 125(4): 1678-1685.

Steinman, L. (2007). "A brief history of $T(H) 17$, the first major revision in the $T(H) 1 / T(H) 2$ hypothesis of T cell-mediated tissue damage." Nat Med 13(2): 139-145.

Steinman, R. M. (1991). "The dendritic cell system and its role in immunogenicity." Annu Rev Immunol 9: 271-296.

Steinman, R. M. (2008). "Dendritic cells in vivo: a key target for a new vaccine science." Immunity 29(3): 319-324.

Steinman, R. M. and J. Banchereau (2007). "Taking dendritic cells into medicine." Nature 449(7161): 419-426.

Steinman, R. M. and Z. A. Cohn (1973). "Identification of a novel cell type in peripheral lymphoid organs of mice. I. Morphology, quantitation, tissue distribution." J Exp Med 137(5): 1142-1162.

Steinman, R. M. and Z. A. Cohn (2007). "Pillars Article: Identification of a novel cell type in peripheral lymphoid organs of mice. I. Morphology, quantitation, tissue distribution. J. Exp. Med.1973. 137: 1142-1162." J Immunol 178(1): 5-25.

Steinman, R. M., D. Hawiger and M. C. Nussenzweig (2003). "Tolerogenic dendritic cells." Annu Rev Immunol 21: 685-711. 
Steinman, R. M. and J. Idoyaga (2010). "Features of the dendritic cell lineage." Immunol Rev 234(1): 5-17.

Steinman, R. M. and M. D. Witmer (1978). "Lymphoid dendritic cells are potent stimulators of the primary mixed leukocyte reaction in mice." Proc Natl Acad Sci U S A 75(10): 51325136.

Stockinger, B. and M. Veldhoen (2007). "Differentiation and function of Th17 T cells." Curr Opin Immunol 19(3): 281-286.

Street, S. E., E. Cretney and M. J. Smyth (2001). "Perforin and interferon-gamma activities independently control tumor initiation, growth, and metastasis." Blood 97(1): 192-197.

Tangye, S. G., D. T. Avery, E. K. Deenick and P. D. Hodgkin (2003). "Intrinsic differences in the proliferation of naive and memory human $B$ cells as a mechanism for enhanced secondary immune responses." J Immunol 170(2): 686-694.

Tedder, T. F., A. W. Boyd, A. S. Freedman, L. M. Nadler and S. F. Schlossman (1985). "The B cell surface molecule B1 is functionally linked with B cell activation and differentiation." J Immunol 135(2): 973-979.

Theurich, S., J. Malcher, H. J. Becker, J. M. Chemnitz, T. M. Liebig, A. ShimabukuroVornhagen, et al. (2011). "Activated primary human B cells efficiently induce early CD4OL and CD107a expression in CD4+ T cells." Blood 118(22): 5979-5980.

Theurich, S., J. Malcher, K. Wennhold, A. Shimabukuro-Vornhagen, J. Chemnitz, U. Holtick, et al. (2013). "Brentuximab vedotin combined with donor lymphocyte infusions for early relapse of Hodgkin lymphoma after allogeneic stem-cell transplantation induces tumorspecific immunity and sustained clinical remission." J Clin Oncol 31(5): e59-63.

Theurich, S., M. Schlaak, H. Steguweit, L. C. Heukamp, K. Wennhold, P. Kurschat, et al. (in press). "Targeting tumor infiltrating B cells in cutaneous T-cell lymphoma." J Clin Oncol.

Theurich, S., K. Wennhold, I. Wedemeyer, A. Rothe, K. Hubel, A. Shimabukuro-Vornhagen, et al. (2013). "CD30-targeted therapy with brentuximab vedotin and DLI in a patient with T-cell posttransplantation lymphoma: induction of clinical remission and cellular immunity." Transplantation 96(3): e16-18.

Thomas, L. (1982). "On immunosurveillance in human cancer." Yale J Biol Med 55(3-4): 329333.

Thorbecke, G. J., A. R. Amin and V. K. Tsiagbe (1994). "Biology of germinal centers in lymphoid tissue." FASEB J 8(11): 832-840. 
Tong, A. W. and M. J. Stone (2003). "Prospects for CD40-directed experimental therapy of human cancer." Cancer Gene Ther 10(1): 1-13.

Topalian, S. L., F. S. Hodi, J. R. Brahmer, S. N. Gettinger, D. C. Smith, D. F. McDermott, et al. (2012). "Safety, activity, and immune correlates of anti-PD-1 antibody in cancer." N Engl J Med 366(26): 2443-2454.

Townsend, S. E., C. C. Goodnow and R. J. Cornall (2001). "Single epitope multiple staining to detect ultralow frequency B cells." J Immunol Methods 249(1-2): 137-146.

Tretter, T., R. K. Venigalla, V. Eckstein, R. Saffrich, S. Sertel, A. D. Ho, et al. (2008). "Induction of CD4+ T-cell anergy and apoptosis by activated human B cells." Blood 112(12): 4555-4564.

van de Ven, A. A., E. B. Compeer, A. C. Bloem, L. van de Corput, M. van Gijn, J. M. van Montfrans, et al. (2012). "Defective calcium signaling and disrupted CD20-B-cell receptor dissociation in patients with common variable immunodeficiency disorders." J Allergy Clin Immunol 129(3): 755-761 e757.

Van den Bosch, G. A., P. Ponsaerts, G. Nijs, M. Lenjou, G. Vanham, D. R. Van Bockstaele, et al. (2005). "Ex vivo induction of viral antigen-specific CD8 T cell responses using mRNAelectroporated CD40-activated B cells." Clin Exp Immunol 139(3): 458-467.

van den Broek, M. E., D. Kagi, F. Ossendorp, R. Toes, S. Vamvakas, W. K. Lutz, et al. (1996). "Decreased tumor surveillance in perforin-deficient mice." J Exp Med 184(5): 1781-1790.

van Kooten, C. and J. Banchereau (2000). "CD40-CD40 ligand." J Leukoc Biol 67(1): 2-17.

van Zelm, M. C. (2012). "Human CD27+lgM+lgD+ B cells: T-cell or TLR-dependent?" Blood 120(25): 4905-4906.

van Zelm, M. C., S. J. Bartol, G. J. Driessen, F. Mascart, I. Reisli, J. L. Franco, et al. (2014). "Human CD19 and CD40L deficiencies impair antibody selection and differentially affect somatic hypermutation." J Allergy Clin Immunol 134(1): 135-144.

von Andrian, U. H. and T. R. Mempel (2003). "Homing and cellular traffic in lymph nodes." Nat Rev Immunol 3(11): 867-878.

von Bergwelt-Baildon, M., J. L. Schultze, B. Maecker, I. Menezes and L. M. Nadler (2004). "Correspondence re R. Lapointe et al., CD40-stimulated B lymphocytes pulsed with tumor antigens are effective antigen-presenting cells that can generate specific $T$ cells. Cancer Res 2003;63:2836-43." Cancer Res 64(11): 4055-4056; author reply 4056-4057. 
von Bergwelt-Baildon, M., A. Shimabukuro-Vornhagen, A. Popov, N. Klein-Gonzalez, F. Fiore, S. Debey, et al. (2006). "CD40-activated B cells express full lymph node homing triad and induce T-cell chemotaxis: potential as cellular adjuvants." Blood 107(7): 2786-2789.

von Bergwelt-Baildon, M. S., R. H. Vonderheide, B. Maecker, N. Hirano, K. S. Anderson, M. O. Butler, et al. (2002). "Human primary and memory cytotoxic T lymphocyte responses are efficiently induced by means of CD40-activated B cells as antigen-presenting cells: potential for clinical application." Blood 99(9): 3319-3325.

Vonderheide, R. H., K. T. Flaherty, M. Khalil, M. S. Stumacher, D. L. Bajor, N. A. Hutnick, et al. (2007). "Clinical activity and immune modulation in cancer patients treated with CP-870,893, a novel CD40 agonist monoclonal antibody." J Clin Oncol 25(7): 876-883.

Walker, P. R., P. Saas and P. Y. Dietrich (1998). "Tumor expression of Fas ligand (CD95L) and the consequences." Curr Opin Immunol 10(5): 564-572.

Walshe, C. A., S. A. Beers, R. R. French, C. H. Chan, P. W. Johnson, G. K. Packham, et al. (2008). "Induction of cytosolic calcium flux by CD20 is dependent upon B Cell antigen receptor signaling." J Biol Chem 283(25): 16971-16984.

Ward, S., D. Casey, M. C. Labarthe, M. Whelan, A. Dalgleish, H. Pandha, et al. (2002). "Immunotherapeutic potential of whole tumour cells." Cancer Immunol Immunother 51(7): 351-357.

Ward, S. M., P. Phalora, D. Bradshaw, H. Leyendeckers and P. Klenerman (2008). "Direct ex vivo evaluation of long-lived protective antiviral memory $B$ cell responses against hepatitis $B$ virus." Journal of Infectious Diseases 198(6): 813-817.

Watanabe, S., H. Kagamu, H. Yoshizawa, N. Fujita, H. Tanaka, J. Tanaka, et al. (2003). "The duration of signaling through CD40 directs biological ability of dendritic cells to induce antitumor immunity." J Immunol 171(11): 5828-5836.

Weiner, L. M., R. Surana and S. Wang (2010). "Monoclonal antibodies: versatile platforms for cancer immunotherapy." Nat Rev Immunol 10(5): 317-327.

Weninger, W., N. Manjunath and U. H. von Andrian (2002). "Migration and differentiation of CD8+ T cells." Immunol Rev 186: 221-233.

Wennhold, K., A. Shimabukuro-Vornhagen, S. Theurich and M. von Bergwelt-Baildon (2013). "CD40-activated B cells as antigen-presenting cells: the final sprint toward clinical application." Expert Rev Vaccines 12(6): 631-637. 
Whiteside, T. L. (2008). "The tumor microenvironment and its role in promoting tumor growth." Oncogene 27(45): 5904-5912.

WHO (2015). "Hepatitis B- Prevention Facts." World Health Organization. from http://www.who.int/mediacentre/factsheets/fs204/en/.

Wiesner, M., C. Zentz, C. Mayr, R. Wimmer, W. Hammerschmidt, R. Zeidler, et al. (2008). "Conditional immortalization of human B cells by CD40 ligation." PLoS One 3(1): e1464.

Wing, K. and S. Sakaguchi (2010). "Regulatory T cells exert checks and balances on self tolerance and autoimmunity." Nat Immunol 11(1): 7-13.

Wolchok, J. D., H. Kluger, M. K. Callahan, M. A. Postow, N. A. Rizvi, A. M. Lesokhin, et al. (2013). "Nivolumab plus ipilimumab in advanced melanoma." N Engl J Med 369(2): 122-133.

Wu, C., Y. Liu, Q. Zhao, G. Chen, J. Chen, X. Yan, et al. (2010). "Soluble CD40 ligand-activated human peripheral $B$ cells as surrogated antigen presenting cells: A preliminary approach for anti-HBV immunotherapy." Virol J 7: 370.

Yoon, S. H., H. I. Cho and T. G. Kim (2005). "Activation of B cells using Schneider 2 cells expressing CD40 ligand for the enhancement of antigen presentation in vitro." Exp Mol Med 37(6): 567-574.

Zhang, Y. (2011). "Tunneling-nanotube: A new way of cell-cell communication." Commun Integr Biol 4(3): 324-325.

Zhang, Y., R. Morgan, E. R. Podack and J. Rosenblatt (2013). "B cell regulation of anti-tumor immune response." Immunol Res 57(1-3): 115-124.

Ziegner, M., G. Steinhauser and C. Berek (1994). "Development of antibody diversity in single germinal centers: selective expansion of high-affinity variants." Eur J Immunol 24(10): 23932400.

Zitvogel, L., J. I. Mayordomo, T. Tjandrawan, A. B. DeLeo, M. R. Clarke, M. T. Lotze, et al. (1996). "Therapy of murine tumors with tumor peptide-pulsed dendritic cells: dependence on T cells, B7 costimulation, and T helper cell 1-associated cytokines." J Exp Med 183(1): 8797. 


\section{Attachments}

\section{Erklärung § 4 Abs. 1 Nr. 9}

Ich versichere, dass ich die von mir vorgelegte Dissertation selbstständig angefertigt, die benutzen Quellen und Hilfsmittel vollständig angegeben und die Stellen der Arbeit einschließlich Tabellen, Karten, Abbildungen -, die anderen Werken im Wortlaut oder dem Sinn nach entnommen sind, in jedem Einzelfall als Entlehnung kenntlich gemacht habe; dass diese Dissertation noch keiner anderen Fakultät oder Universität zur Prüfung vorgelegen hat; dass sie - abgesehen von unten angegebenen Teilpublikationen - noch nicht veröffentlicht worden ist sowie, dass ich eine solche Veröffentlichung vor Abschluss des Promotionsverfahrens nicht vornehmen werde. Die Bestimmungen der Promotionsordnung sind mir bekannt. Die von mir vorgelegte Dissertation ist von Prof. Dr. Thorsten Hoppe und Prof. Dr. Dr. Michael von Bergwelt-Baildon betreut worden.

Köln, den 21.04.2015

Kerstin Wennhold

\section{Teilpublikationen}

Nela Klein Gonzalez*, Kerstin Wennhold*, Sandra Balkow, Eisei Kondo, Tanja Weber, Maria Garcia-Marquez, Stephan Grabbe, Wilhelm Bloch, Michael von Bergwelt-Baildon*, Alexander Shimabukuro-Vornhagen*. 'In vitro and in vivo imaging of initial T-B cell interactions in the setting of B cell-based cancer immunotherapy.' Oncoimmunology. Accepted 2015. * Contributed equally.

Kerstin Wennhold, Martin Thelen, Maria Garcia-Marquez, Alexander ShimabukuroVornhagen, Michael von Bergwelt-Baildon. ,Tumorantigen-specific CD4OB cells: Combining enhanced antigen presentation and antibody-secretion for tumor targeting'. Manuscript in preparation for submission. 